This item was submitted to Loughborough's Research Repository by the author.

Items in Figshare are protected by copyright, with all rights reserved, unless otherwise indicated.

\title{
The stable manifold theorem for semilinear stochastic evolution equations
} and stochastic partial differential equations

PLEASE CITE THE PUBLISHED VERSION

LICENCE

CC BY-NC-ND 4.0

\section{REPOSITORY RECORD}

Mohammed, Salah-Eldin A., Tusheng Zhang, and Huaizhong Zhao. 2019. "The Stable Manifold Theorem for Semilinear Stochastic Evolution Equations and Stochastic Partial Differential Equations". figshare. https://hdl.handle.net/2134/1895. 


\title{
THE STABLE MANIFOLD THEOREM FOR SEMILINEAR STOCHASTIC EVOLUTION EQUATIONS AND STOCHASTIC PARTIAL DIFFERENTIAL EQUATIONS
}

\author{
Salah-Eldin A. Mohammed*, Tusheng Zhang** and Huaizhong Zhao***
}

\begin{abstract}
The main objective of this paper is to characterize the pathwise local structure of solutions of semilinear stochastic evolution equations (see's) and stochastic partial differential equations (spde's) near stationary solutions. Such characterization is realized through the long-term behavior of the solution field near stationary points. The analysis falls in two parts 1, 2. In Part 1 , we prove general existence and compactness theorems for $C^{k}$-cocycles of semilinear see's and spde's. Our results cover a large class of semilinear see's as well as certain semilinear spde's with Lipschitz and non-Lipschitz terms such as stochastic reaction diffusion equations and the stochastic Burgers equation with additive infinite-dimensional noise. In Part 2, stationary solutions are viewed as cocycle-invariant random points in the infinite-dimensional state space. The pathwise local structure of solutions of semilinear see's and spde's near stationary solutions is described in terms of the almost sure long-time behavior of trajectories of the equation in relation to the stationary solution. More specifically, we establish local stable manifold theorems for semilinear see's and spde's (Theorems 2.4.1-2.4.4). These results give smooth stable and unstable manifolds in the neighborhood of a hyperbolic stationary solution of the underlying stochastic equation. The stable and unstable manifolds are stationary, live in a stationary tubular neighborhood of the stationary solution and are asymptotically invariant under the stochastic semiflow of the see/spde. Furthermore, the local stable and unstable manifolds intersect transversally at the stationary point, and the unstable manifolds has fixed finite dimension. The proof uses infinite-dimensional multiplicative ergodic theory techniques, interpolation and perfection arguments (Theorem 2.2.1).
\end{abstract}

\footnotetext{
*The research of this author is supported in part by NSF Grants DMS-9703852, DMS-9975462 and DMS-0203368.

** The research of this author is supported in part by EPSRC Grant GR/R91144.

*** The research of this author is supported in part by EPSRC Grants GR/R69518 and GR/R93582.

AMS 1991 subject classifications. Primary 60H10, 60H20; secondary 60H25.

Key words and phrases. Stochastic semiflow, $C^{k}$ cocycle, stochastic evolution equation (see), stochastic partial differential equation (spde), multiplicative ergodic theorem, stationary solution, hyperbolicity, local stable (unstable) manifolds.
} 


\section{Table of Contents}

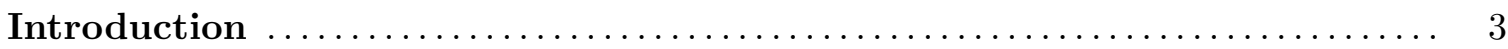

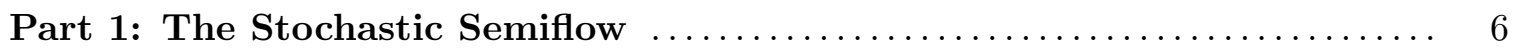

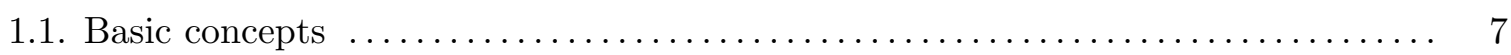

1.2. Flows and cocycles of semilinear stochastic evolution equations $\ldots \ldots \ldots \ldots \ldots \ldots \quad 8$

1.3. Semilinear stochastic partial differential equations: Lipschitz nonlinearity $\ldots \ldots \ldots 37$

1.4. Semilinear stochastic partial differential equations: Non-Lipschitz Nonlinearity .. 46

Part 2: Existence of Stable and Unstable Manifolds $\ldots \ldots \ldots \ldots \ldots \ldots \ldots \ldots$. 68



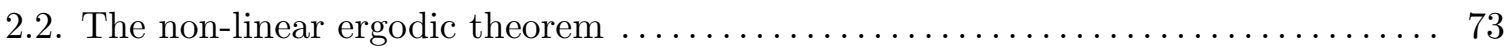

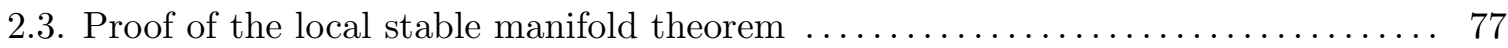

2.4. The local stable manifold theorem for see's and spde's $\ldots \ldots \ldots \ldots \ldots \ldots \ldots \ldots$

Acknowledgments ................................................ 103

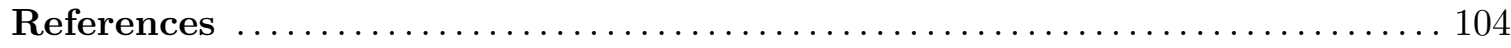




\section{Introduction}

The construction of local stable and unstable manifolds near hyperbolic equilibria is a fundamental problem in deterministic and stochastic dynamical systems. The significance of these invariant manifolds consists in a characterization of the local behavior of the dynamical system in terms of long-time asymptotics of its trajectories near a stationary point. In recent years, it has been established that local stable/unstable manifolds exist for finite-dimensional stochastic ordinary differential equations (sode's) ([M-S.2]) and stochastic systems with finite memory (sfde's)([M-S.1]). On the other hand, existence of such manifolds for non-linear stochastic evolution equations (see's) and stochastic partial differential equations (spde's) has been an open problem since the early nineties ([F-S], [B-F], [B-F.1]).

In [F-S], Flandoli and Schaumlöffel established the existence of a random evolution operator and its Lyapunov spectrum for a linear stochastic heat equation on a bounded Euclidean domain, driven by finite-dimensional white noise. For linear see's with finite-dimensional white noise, a stochastic semi-flow (i.e. random evolution operator) was obtained in [B-F]. Subsequent work on the dynamics of non-linear spde's has focused mainly on the question of existence of continuous semiflows and the existence and uniqueness of invariant measures and/or stationary solutions. Recent results on the existence of global invariant, stable/unstable manifolds (through a fixed point) for semilinear see's are obtained by Duan, Lu and Schmalfuss ([D-L-S.1],[D-L-S.2]). The results in ([D-L-S.1], [D-L-S.1]) assume that the see is driven by multiplicative one-dimensional Brownian motion, with the nonlinear term having a global Lipschitz constant that is sufficiently small relative to the sectral gaps of the second-order linear operator. The latter spectral gap condition in ([D-L-S.1], [D-L-S.2]) is dictated by the use of the contraction mapping theorem.

The main objective of this article is to establish the existence of local stable and unstable manifolds near stationary solutions of semilinear stochastic evolution equations (see's) and stochastic partial differential equations (spde's). Our approach consists in the following two major undertakings:

- A construction of a sufficiently Fréchet differentiable cocycle for mild/weak trajectories of the see or the spde. This is achieved in the see case by a combination of a chaos-type expansion and suitable lifting techniques, and for spde's by using stochastic variational representations and methods from deterministic pde's. Part I of this paper is devoted to detailing the construction of the cocycle.

- The application of classical non-linear ergodic theory techniques developed by Oseledec [O] and Ruelle [Ru.2] in order to study the local structure of the above cocycle in a neighborhood of a hyperbolic stationary point. Stationary points correspond to stationary solutions and hyperbolicity is characterized via the Lyapunov spectrum of the linearized cocycle along the stationary trajectory. This is the subject of Part 2 of this article. 
The problem of existence of semiflows for see's and spde's is a non-trivial one, mainly due to the well-established fact that finite-dimensional methods for constructing (even continuous) stochastic flows break down in the infinite-dimensional setting of spde's and see's. In particular, Kolmogorov's continuity theorem fails for random fields parametrized by infinite-dimensional Hilbert spaces ([Mo.1], pp. 144-149, [Sk], [Mo.2]). (Cf. also [F.1], [F.2], [D-Z.1], pp. 246-248). In view of the failure of Kolmogorov's theorem, issues of perfection of the infinite-dimensional semiflow and its ergodic properties are of prime importance because it is necessary to extend Ruelle's discrete-time multiplicative ergodic theory to a continuous time setting. On the other hand, there is a significant body of literature on the existence of perfect finite-dimensional stochastic flows and cocycles generated by stochastic ordinary differential equations in Euclidean space or finitedimensional manifolds. The reader may refer to [C], [A], [A-S], [M-S.2], [M-S.3], [M-S.4], [Ku] and [I-W] and the references therein. Needless to say that, in these works, Kolmogorov's continuity theorem plays a central role in the construction of the underlying finite-dimensional stochastic flows.

In Part 1 of this article, we show the existence of smooth perfect cocycles for mild solutions of semilinear see's in Hilbert space (Theorem 1.2.6). Our construction employs a "chaos-type" representation in the Hilbert-Schmidt operators, using the linear terms of the see (Theorems 1.2.11.2.4). This technique bypasses the need for Kolmogorov's continuity theorem and appears to be new. A variational technique is then employed in order to handle the non-linear terms. Applications to specific classes of spde's are given. In particular, we obtain smooth stochastic semiflows for semilinear spde's driven by cylindrical Brownian motion with a covariance Hilbert space $K$ (Theorem 1.3.5). In these applications, it turns out that in addition to smoothness of the non-linear terms, one requires some level of dissipativity or Lipschitz continuity in order to guarantee the existence of smooth globally defined semiflows. Specific examples of spde's include semilinear parabolic spde's with Lipschitz nonlinearities (Theorem 1.3.5), stochastic reaction diffusion equations (Theorems 1.4.1, 1.4.2) and stochastic Burgers equations with additive infinite-dimensional noise (Theorem 1.4.3).

As indicated above, the existence of a smooth semiflow is a necessary tool for constructing the stable and unstable manifolds for see's and spde's near a hyperbolic stationary random point, ala work of Oseledec-Ruelle ([O], [Ru.1], [Ru.2]). This issue is the main objective of the analysis in Part 2 of this article. Hyperbolicity of a stationary point is defined via the Lyapunov spectrum of the linearization of the perfect cocycle constructed in Part 1. Using Kingman's subadditive ergdoic theorem and Ruelle's discrete non-linear multiplicative ergodic theory techniques, appropriate estimates are developed in order to control the excursions of the nonlinear cocycle between discrete time points. Thus, perfect versions of the local stable and unstable manifolds are constructed within a stationary neighborhood of the hyperbolic equilibrium. Furthermore, it is shown in this part that the local stable/unstable manifolds are transveral and asymptotically invariant under the non-linear cocycle, and the unstable manifolds are finite-dimensional with fixed (non-random) dimension. These results are referred to collectively as local stable manifold theorems. Local stable manifold theorems are established for various classes of semilinear see's and spde's (Theorems 
2.4.1-2.4.4). In particular, our results cover semilinear stochastic evolution equations, stochastic parabolic equations, stochastic reaction-diffusion equations, and Burgers equation with additive infinite-dimensional noise in $L^{2}([0,1]$.

From the viewpoint of spde's, the results in this article go well beyond standard issues of existence, uniqueness of mild/weak solutions, existence and ergodicity of the invariant measure. These fundamental issues have occupied the spde community for a long time and are well-studied (cf. for example [Wa], [D-Z.1], [D-Z.2], [Si.1], [Si.2], [C-K-S]). Since the main objective of the present article is to characterize "generic" a.s. local behavior of the stochastic semiflow near equilibria, emphasis is placed largely on issues of hyperbolicity of the stationary solutions rather than existence and uniqueness/ergodicity of the invariant measure. From a dynamical systems point of view, it is needless to say that ergodicity of the stationary solution is a non-generic property. In general, as in the deterministic case, finite and infinite-dimensional stochastic dynamical systems admit more than one stationary point. The stochastic dynamics in a regime with multiple equilibria is not well-understood.

Finally, it should be noted that the case of non-linear multiplicative noise is largely open: It is not known to us if see's driven by non-linear multidimensional white noise admit perfect (smooth, or even continuous) cocycles. The issue of existence of stationary solutions for spde's driven by non-linear white noise and their relation to backward sde's is being investigated in [Z-Z]. 


\section{Part 1: The Stochastic Semiflow}

\subsection{Basic concepts.}

This part of our article is devoted to the contruction of Fréchet smooth stochastic semiflows for mild and weak solutions of semilinear see's and spde's.

In Theorem 1.2.6, it is shown that mild solutions of semilinear see's in a Hilbert space $H$ generate smooth perfect compacting cocycles. The construction of the cocycle for semilinear see's is based on the following new strategy, which bypasses the need for Kolmogorov's continuity theorem:

- We "lift" the linear terms of the see to the Hilbert-Schmidt operators $L_{2}(H)$.

- We represent the mild solution of the linear see as a "chaos-type" series expansion living in the Hilbert space $L_{2}(H)$ of Hilbert-Schmidt operators on $H$.

- Using a variational technique, the mild solution of the full semilinear see is represented in terms of the linear cocycle constructed above (Theorems 1.2.1-1.2.4). This part of the strategy requires the non-linear part of the see to satisfy a Lipschitz condition (Theorem 1.3.5).

Similar variational techniques are used to construct smooth cocycles for weak solutions of specific classes of spde's. In particular, we obtain smooth stochastic semiflows for semilinear spde's driven by cylindrical Brownian motion with a covariance Hilbert space $K$. In these applications, it turns out that in addition to smoothness of the non-linear terms, one requires some level of dissipativity or Lipschitz continuity, e.g. the stochastic heat equation (Theorem 1.3.5), the stochastic reaction diffusion equation (Theorems 1.4.1, 1.4.2) and stochastic Burgers equations with additive infinite-dimensional noise (Theorem 1.4.3).

We begin by formulating the ideas of a stochastic semiflow and a cocycle which are central to the analysis in this work.

Let $(\Omega, \mathcal{F}, P)$ be a probability space. Denote by $\overline{\mathcal{F}}$ the $P$-completion of $\mathcal{F}$, and let $\left(\Omega, \overline{\mathcal{F}},\left(\mathcal{F}_{t}\right)_{t \geq 0}, P\right)$ be a complete filtered probability space satisfying the usual conditions $([\operatorname{Pr}])$. Denote $\Delta:=\left\{(s, t) \in \mathbf{R}^{2}: 0 \leq s \leq t\right\}$, and $\mathbf{R}^{+}:=[0, \infty)$. For a topological space $E$, let $\mathcal{B}(E)$ denote its Borel $\sigma$-algebra.

Let $k$ be a positive integer and $0<\epsilon \leq 1$. If $E, N$ are real Banach spaces, we will denote by $L^{(k)}(E, N)$ the Banach space of all $k$-multilinear maps $A: E^{k} \rightarrow N$ with the uniform norm $\|A\|:=\sup \left\{\left|A\left(v_{1}, v_{2}, \cdots, v_{k}\right)\right|: v_{i} \in E,\left|v_{i}\right| \leq 1, i=1, \cdots, k\right\}$. Suppose $U \subseteq E$ is an open set. A map $f: U \rightarrow N$ is said to be of class $C^{k, \epsilon}$ if it is $C^{k}$ and if $D^{(k)} f: U \rightarrow L^{(k)}(E, N)$ is $\epsilon$-Hölder continuous on bounded sets in $U$. A $C^{k, \epsilon} \operatorname{map} f: U \rightarrow N$ is said to be of class $C_{b}^{k, \epsilon}$ if all its derivatives $D^{(j)} f, 1 \leq j \leq k$, are globally bounded on $U$, and $D^{(k)} f$ is $\epsilon$-Hölder continuous on $U$. A mapping $\tilde{f}:[0, T] \times U \rightarrow N$ is of class $C^{k, \epsilon}$ in the second variable uniformly with respect to the first if for each $t \in[0, T], \tilde{f}(t, \cdot)$ is $C^{k, \epsilon}$ on $U$, for every bounded set $U_{0} \subseteq U$ the spatial partial derivatives $D^{(j)} \tilde{f}(t, x), j=1, \cdots, k$, are uniformly bounded in $(t, x) \in[0, T] \times U_{0}$ and the corresponding $\epsilon$-Hölder constant of $D^{(k)} \tilde{f}(t, \cdot) \mid U_{0}$ is uniformly bounded in $t \in[0, T]$. 
The following definitions are crucial to the developments in this article.

\section{Definition 1.1.1.}

Let $E$ be a Banach space, $k$ a non-negative integer and $\epsilon \in(0,1]$. A stochastic $C^{k, \epsilon}$ semiflow on $E$ is a random field $V: \Delta \times E \times \Omega \rightarrow E$ satisfying the following properties:

(i) $V$ is $(\mathcal{B}(\Delta) \otimes \mathcal{B}(E) \otimes \mathcal{F}, \mathcal{B}(E))$-measurable.

(ii) For each $\omega \in \Omega$, the map $\Delta \times E \ni(s, t, x) \mapsto V(s, t, x, \omega) \in E$ is continuous.

(iii) For fixed $(s, t, \omega) \in \Delta \times \Omega$, the map $E \ni x \mapsto X(s, t, x, \omega) \in E$ is $C^{k, \epsilon}$.

(iv) If $0 \leq r \leq s \leq t, \omega \in \Omega$ and $x \in E$, then

$$
V(r, t, x, \omega)=V(s, t, V(r, s, x, \omega), \omega)
$$

(v) For all $(s, x, \omega) \in \mathbf{R}^{+} \times E \times \Omega$, one has $V(s, s, x, \omega)=x$.

\section{Definition 1.1.2.}

Let $\theta: \mathbf{R}^{+} \times \Omega \rightarrow \Omega$ be a $P$-preserving semigroup on the probability space $(\Omega, \mathcal{F}, P), E$ a Banach space, $k$ a non-negative integer and $\epsilon \in(0,1]$. A $C^{k, \epsilon}$ perfect cocycle $(U, \theta)$ on $E$ is a $\left(\mathcal{B}\left(\mathbf{R}^{+}\right) \otimes \mathcal{B}(E) \otimes \mathcal{F}, \mathcal{B}(E)\right)$-measurable random field $U: \mathbf{R}^{+} \times E \times \Omega \rightarrow E$ with the following properties:

(i) For each $\omega \in \Omega$, the map $\mathbf{R}^{+} \times E \ni(t, x) \mapsto U(t, x, \omega) \in E$ is continuous; and for fixed $(t, \omega) \in \mathbf{R}^{+} \times \Omega$, the map $E \ni x \mapsto U(t, x, \omega) \in E$ is $C^{k, \epsilon}$.

(ii) $U(t+s, \cdot, \omega)=U(t, \cdot, \theta(s, \omega)) \circ U(s, \cdot, \omega)$ for all $s, t \in \mathbf{R}^{+}$and all $\omega \in \Omega$.

(iii) $U(0, x, \omega)=x$ for all $x \in E, \omega \in \Omega$.

Note that a cocycle $(U, \theta)$ corresponds to a one-parameter semigroup on $E \times \Omega$, viz.

$$
\begin{aligned}
\mathbf{R}^{+} \times E \times \Omega & \rightarrow E \times \Omega \\
(t,(x, \omega)) & \mapsto(U(t, x, \omega), \theta(t, \omega))
\end{aligned}
$$

Fig.1 illustrates the cocycle property. The vertical solid lines represent random copies of $E$ sampled according to the probability measure $P$. 


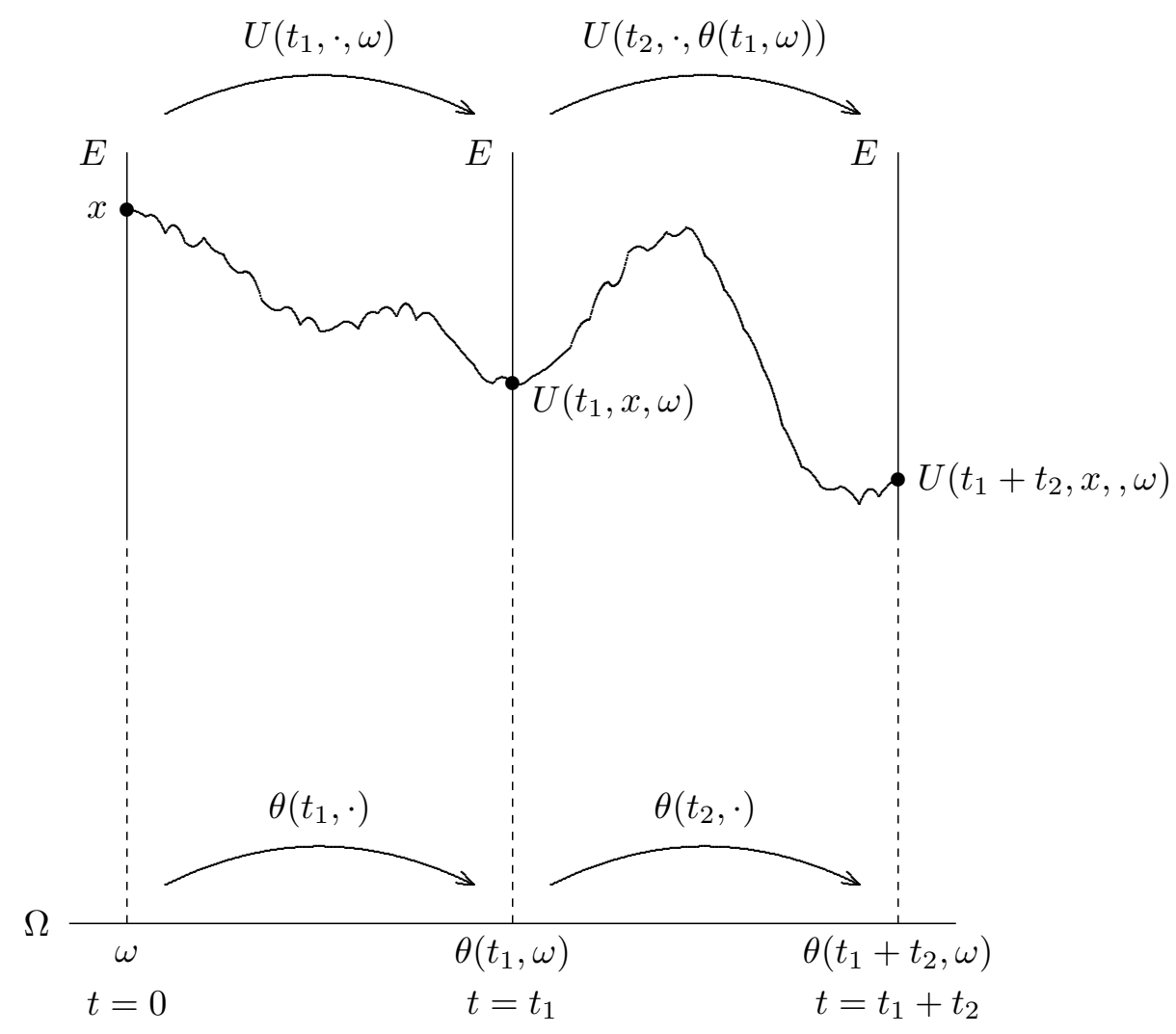

Fig.1: The Cocycle Property

The main objective of this part of our article is to show that under sufficient regularity conditions on the coefficients, a large class of semilinear see's and spde's admits a $C^{k, \epsilon}$ semiflow $V: \Delta \times H \times \Omega \rightarrow H$ for a suitably chosen state space $H$ and which satisfies $V\left(t_{0}, t, x, \cdot\right)=u(t, x)$ for all $x \in H$ and $t \geq t_{0}$, a.s., where $u$ is the solution of the see/spde with initial function $x \in H$ at $t=t_{0}$. In the autonomous case, we show further that the semiflow $V$ generates a cocycle $(U, \theta)$ on $H$, in the sense of Definition 1.1.2 above. The cocycle and its Fréchet derivative are compact in all cases.

\subsection{Flows and cocycles of semilinear stochastic evolution equations.}

In this section, we will establish the existence and regularity of semiflows generated by mild solutions of semilinear stochastic evolution equations. We will begin with the linear case. In fact, the linear cocycle will be used to represent the mild solution of the semilinear stochastic evolution equation via a variational formula which transforms the semilinear stochastic evolution equation to a random integral equation (Theorem 1.2.5). The latter equation plays a key role in establishing the regularity of the stochastic flow of the semilinear see (Theorem 1.2.6). 
One should note at this point the fact that Kolmogorov's continuity theorem fails for random fields parametrized by infinite-dimensional spaces. As a simple example, consider the random field $I: L^{2}([0,1], \mathbf{R}) \rightarrow L^{2}(\Omega, \mathbf{R})$ defined by the Wiener integral

$$
I(x):=\int_{0}^{1} x(t) d W(t), \quad x \in L^{2}([0,1], \mathbf{R}) .
$$

The above random field has no continuous (or even linear!) measurable selection $L^{2}([0,1], \mathbf{R}) \times \Omega \rightarrow$ R ([Mo.1], pp. 144-148; [Mo.2]).

\section{(a) Linear stochastic evolution equations}

We will first prove the existence of semiflows associated with mild solutions of linear stochastic evolution equations of the form:

$$
\left.\begin{array}{l}
d u(t, x, \cdot)=-A u(t, x, \cdot) d t+B u(t, x, \cdot) d W(t), \quad t>0 \\
u(0, x, \omega)=x .
\end{array}\right\}
$$

In the above equation $A: D(A) \subset H \rightarrow H$ is a closed linear operator on a separable real Hilbert space $H$. Assume that $A$ has a complete orthonormal system of eigenvectors $\left\{e_{n}: n \geq 1\right\}$ with corresponding positive eigenvalues $\left\{\mu_{n}, n \geq 1\right\}$; i.e., $A e_{n}=\mu_{n} e_{n}, n \geq 1$. Suppose $-A$ generates a strongly continuous semigroup of bounded linear operators $T_{t}: H \rightarrow H, t \geq 0$. Let $E$ be a separable Hilbert space and $W(t), t \geq 0$, be an E-valued Brownian motion defined on the canonical filtered Wiener space $\left(\Omega, \mathcal{F},\left(\mathcal{F}_{t}\right)_{t \geq 0}, P\right)$ and with a separable covariance Hilbert space $K$. Here $K \subset E$ is a Hilbert-Schmidt embedding. Indeed, $\Omega$ is the space of all continuous paths $\omega: \mathbf{R} \rightarrow E$ such that $\omega(0)=0$ with the compact open topology, $\mathcal{F}$ is its Borel $\sigma$-field, $\mathcal{F}_{t}$ is the sub- $\sigma$-field of $\mathcal{F}$ generated by all evaluations $\Omega \ni \omega \mapsto \omega(u) \in E, u \leq t$, and $P$ is Wiener measure on $\Omega$. The Brownian motion is given by

$$
W(t, \omega):=\omega(t), \quad \omega \in \Omega, t \in \mathbf{R},
$$

and may be represented by

$$
W(t)=\sum_{k=1}^{\infty} W^{k}(t) f_{k}, \quad t \in \mathbf{R},
$$

where $\left\{f_{k}: k \geq 1\right\}$ is a complete orthonormal basis of $K$, and $W^{k}, k \geq 1$, are standard independent one-dimensional Wiener processes ([D-Z.1], Chapter 4). Note that, in general, the above series converges in $E$ but not in $K$.

Denote by $L_{2}(K, H) \subset L(K, H)$ the Hilbert space of all Hilbert-Schmidt operators $S: K \rightarrow$ $H$, given the norm

$$
\|S\|_{2}:=\left[\sum_{k=1}^{\infty}\left|S\left(f_{k}\right)\right|^{2}\right]^{1 / 2},
$$

where $|\cdot|$ is the norm on $H$. Suppose $B: H \rightarrow L_{2}(K, H)$ is a bounded linear operator. The stochastic integral in (1.2.1) is defined in the following sense ([D-Z.1], Chapter 4): 
Let $F:[0, a] \times \Omega \rightarrow L_{2}(K, H)$ be $\mathcal{B}\left([0, a] \otimes \mathcal{F}, \mathcal{B}\left(L_{2}(K, H)\right)\right)$-measurable, $\left(\mathcal{F}_{t}\right)_{t \geq 0}$-adapted and such that $\int_{0}^{a} E\|F(t)\|_{L_{2}(K, H)}^{2} d t<\infty$. Define

$$
\int_{0}^{a} F(t) d W(t):=\sum_{k=1}^{\infty} \int_{0}^{a} F(t)\left(f_{k}\right) d W^{k}(t)
$$

where the $H$-valued stochastic integrals on the right hand side are with respect to the onedimensional Wiener processes $W^{k}, k \geq 1$. Note that the above series converges in $L^{2}(\Omega, H)$ because

$$
\sum_{k=1}^{\infty} E\left|\int_{0}^{a} F(t)\left(f_{k}\right) d W^{k}(t)\right|^{2}=\int_{0}^{a} E\|F(t)\|_{L_{2}(K, H)}^{2} d t<\infty .
$$

Throughout the rest of the article, we will denote by $\theta: \mathbf{R} \times \Omega \rightarrow \Omega$ the standard $P$ preserving ergodic Wiener shift on $\Omega$ :

$$
\theta(t, \omega)(s):=\omega(t+s)-\omega(t), \quad t, s \in \mathbf{R} .
$$

Hence $(W, \theta)$ is a helix:

$$
W\left(t_{1}+t_{2}, \omega\right)-W\left(t_{1}, \omega\right)=W\left(t_{2}, \theta\left(t_{1}, \omega\right)\right), \quad t_{1}, t_{2} \in \mathbf{R}, \omega \in \Omega .
$$

As usual, we let $L(H)$ be the Banach space of all bounded linear operators $H \rightarrow H$ given the uniform operator norm $\|\cdot\|_{L(H)}$. Denote by $L_{2}(H) \subset L(H)$ the Hilbert space of all Hilbert-Schmidt operators $S: H \rightarrow H$. It is easy to see that if $S \in L_{2}(H)$ and $T \in L(H)$, then $\|S\|_{L(H)} \leq\|S\|_{2}$, $T \circ S($ and $S \circ T) \in L_{2}(H)$ and $\|T \circ S\|_{L_{2}(H)} \leq\|T\|_{L(H)}\|S\|_{L_{2}(H)}$.

A mild solution of (1.2.1) is a family of $\left(\mathcal{B}\left(\mathbf{R}^{+}\right) \otimes \mathcal{F}, \mathcal{B}(H)\right)$-measurable, $\left(\mathcal{F}_{t}\right)_{t \geq 0}$-adapted processes $u(\cdot, x, \cdot): \mathbf{R}^{+} \times \Omega \rightarrow H, x \in H$, satisfying the following stochastic integral equation:

$$
u(t, x, \cdot)=T_{t} x+\int_{0}^{t} T_{t-s} B u(s, x, \cdot) d W(s), \quad t \geq 0 .
$$

The next lemma describes a canonical lifting of the strongly continuous semigroup $T_{t}$ : $H \rightarrow H, t \geq 0$, to a strongly continuous semigroup of bounded linear operators $\tilde{T}_{t}: L_{2}(K, H) \rightarrow$ $L_{2}(K, H), t \geq 0$.

\section{Lemma 1.2.1.}

Define the family of maps $\tilde{T}_{t}: L_{2}(K, H) \rightarrow L_{2}(K, H), t \geq 0$, by

$$
\tilde{T}_{t}(C):=T_{t} \circ C, \quad C \in L_{2}(K, H), t \geq 0 .
$$

Then the following is true:

(i) $\tilde{T}_{t}, t \geq 0$, is a strongly continuous semigroup of bounded linear operators on $L_{2}(K, H)$; and $\left\|\tilde{T}_{t}\right\|_{L\left(L_{2}(K, H)\right)}=\left\|T_{t}\right\|_{L(H)}$ for all $t \geq 0$. 
(ii) If $\tilde{A}: \mathcal{D}(\tilde{A}) \subset L_{2}(K, H) \rightarrow L_{2}(K, H)$ is the infinitesimal generator of $\tilde{T}_{t}, t \geq 0$, then

$$
\mathcal{D}(\tilde{A})=\left\{C: C \in L_{2}(K, H), C(K) \subseteq \mathcal{D}(A), A \circ C \in L_{2}(K, H)\right\}
$$

and

$$
\tilde{A}(C)=A \circ C
$$

for all $C \in \mathcal{D}(\tilde{A})$.

(iii) $\tilde{T}_{t}, t \geq 0$, is a contraction semigroup if $T_{t}, t \geq 0$, is.

Proof.

Observe that each $\tilde{T}_{t}: L_{2}(K, H) \rightarrow L_{2}(K, H), t \geq 0$, is a bounded linear map of $L_{2}(K, H)$ into itself. Indeed, it is easy to see that

$$
\left\|\tilde{T}_{t}(C)\right\|_{L_{2}(K, H)} \leq\left\|T_{t}\right\|_{L(H)}\|C\|_{L_{2}(K, H)}, \quad C \in L_{2}(K, H), t \geq 0
$$

and hence $\left\|\tilde{T}_{t}\right\|_{L\left(L_{2}(K, H)\right)} \leq\left\|T_{t}\right\|_{L(H)}$ for all $t \geq 0$. This implies assertion (iii). The reverse inequality

$$
\left\|T_{t}\right\|_{L(H)} \leq\left\|\tilde{T}_{t}\right\|_{L\left(L_{2}(K, H)\right)}, \quad t \geq 0
$$

is not hard to check. Hence the last assertion in (i) holds.

We next verify the semi-group property of $\tilde{T}_{t}, t \geq 0$. Let $t_{1}, t_{2} \geq 0, C \in L_{2}(K, H)$. Then

$$
\left(\tilde{T}_{t_{2}} \circ \tilde{T}_{t_{1}}\right)(C)=T_{t_{2}} \circ\left(T_{t_{1}} \circ C\right)=T_{t_{1}+t_{2}} \circ C=\tilde{T}_{t_{1}+t_{2}}(C) .
$$

Note also that $\tilde{T}_{0}=I_{L\left(L_{2}(K, H)\right)}$, the identity map $L_{2}(K, H) \rightarrow L_{2}(K, H)$. Therefore, $\tilde{T}_{t}, t \geq 0$, is a semigroup on $L_{2}(K, H)$. To prove the strong continuity of $\tilde{T}_{t}, t \geq 0$, we will show that

$$
\lim _{t \rightarrow 0+} \tilde{T}_{t}(C)=C
$$

for each $C \in L_{2}(K, H)$. To prove the above relation, let $C \in L_{2}(K, H)$ and recall that $\left\{f_{k}: k \geq 1\right\}$ is a complete orthonormal basis of $K$. From the strong continuity of $T_{t}, t \geq 0$, it follows that

$$
\lim _{t \rightarrow 0+}\left|T_{t}\left(C\left(f_{k}\right)\right)-C\left(f_{k}\right)\right|_{H}^{2}=0
$$

for each integer $k \geq 1$. Furthermore,

$$
\left|T_{t}\left(C\left(f_{k}\right)\right)-C\left(f_{k}\right)\right|_{H}^{2} \leq 2\left[\sup _{0 \leq t \leq a}\left\|T_{t}\right\|_{L(H)}^{2}+1\right]\left|C\left(f_{k}\right)\right|_{H}^{2}, \quad k \geq 1 .
$$

Since $C$ is Hilbert-Schmidt, (1.2.6) implies that the series $\sum_{k=1}^{\infty}\left|T_{t}\left(C\left(f_{k}\right)\right)-C\left(f_{k}\right)\right|_{H}^{2}$ converges uniformly with respect to $t$. Therefore, from (1.2.5), (1.2.6) and dominated convergence, it follows that

$$
\begin{aligned}
\lim _{t \rightarrow 0+}\left\|\tilde{T}_{t}(C)-C\right\|_{L_{2}(K, H)}^{2} & =\lim _{t \rightarrow 0+} \sum_{k=1}^{\infty}\left|T_{t}\left(C\left(f_{k}\right)\right)-C\left(f_{k}\right)\right|_{H}^{2} \\
& =\sum_{k=1}^{\infty} \lim _{t \rightarrow 0+}\left|T_{t}\left(C\left(f_{k}\right)\right)-C\left(f_{k}\right)\right|_{H}^{2}=0
\end{aligned}
$$


Therefore, (1.2.4) holds and $\tilde{T}_{t}, t \geq 0$, is strongly continuous.

We next prove assertion (ii) of the lemma. Let $\tilde{A}: \mathcal{D}(\tilde{A}) \subset L_{2}(K, H) \rightarrow L_{2}(K, H)$ be the infinitesimal generator of $\tilde{T}_{t}, t \geq 0$. We begin with a proof of the inclusion

$$
\left\{C: C \in L_{2}(K, H), C(K) \subseteq \mathcal{D}(A), A \circ C \in L_{2}(K, H)\right\} \subseteq \mathcal{D}(\tilde{A})
$$

Let $C \in L_{2}(K, H)$ be such that $C(K) \subseteq \mathcal{D}(A)$ and $A \circ C \in L_{2}(K, H)$. We will show that

$$
\lim _{t \rightarrow 0+} \frac{\tilde{T}_{t}(C)-C}{t}=A \circ C
$$

in $L_{2}(K, H)$. To prove $(1.2 .9)$, note first that

$$
\begin{aligned}
\sup _{0 \leq t \leq a} \frac{1}{t}\left|T_{t}\left(C\left(f_{k}\right)\right)-C\left(f_{k}\right)\right|_{H} & =\sup _{0 \leq t \leq a} \frac{1}{t}\left|\int_{0}^{t} T_{s}\left(A\left(C\left(f_{k}\right)\right)\right) d s\right|_{H} \\
& \leq \sup _{0 \leq t \leq a}\left\|T_{s}\right\|_{L(H)}\left|A\left(C\left(f_{k}\right)\right)\right|_{H}
\end{aligned}
$$

because $C\left(f_{k}\right) \in \mathcal{D}(A)$ for every $k \geq 1$. Since

$$
\|A \circ C\|_{L_{2}(K, H)}=\sum_{k=1}^{\infty}\left|A\left(C\left(f_{k}\right)\right)\right|_{H}^{2}<\infty,
$$

it follows from (1.2.10), (1.2.11) and dominated convergence that

$$
\begin{aligned}
\limsup _{t \rightarrow 0+}\left\|\frac{\tilde{T}_{t}(C)-C}{t}-A \circ C\right\|_{L_{2}(K, H)}^{2} & =\limsup _{t \rightarrow 0+} \sum_{k=1}^{\infty}\left|\frac{T_{t}\left(C\left(f_{k}\right)\right)-C\left(f_{k}\right)}{t}-A\left(C\left(f_{k}\right)\right)\right|_{H}^{2} \\
& \leq \sum_{k=1}^{\infty} \limsup _{t \rightarrow 0+}\left|\frac{T_{t}\left(C\left(f_{k}\right)\right)-C\left(f_{k}\right)}{t}-A\left(C\left(f_{k}\right)\right)\right|_{H}^{2} \\
& =0 .
\end{aligned}
$$

This proves (1.2.9). In particular, $C \in \mathcal{D}(\tilde{A})$ and $\tilde{A}(C)=A \circ C$.

It remains to prove the inclusion

$$
\mathcal{D}(\tilde{A}) \subseteq\left\{C: C \in L_{2}(K, H), C(K) \subseteq \mathcal{D}(A), A \circ C \in L_{2}(K, H)\right\}
$$

Suppose $C \in \mathcal{D}(\tilde{A})$. We will show that $C(K) \subseteq \mathcal{D}(A), A \circ C \in L_{2}(K, H)$ and $\tilde{A}(C)=A \circ C$. Since

$$
\lim _{t \rightarrow 0+}\left\|\frac{\tilde{T}_{t}(C)-C}{t}-\tilde{A}(C)\right\|_{L_{2}(K, H)}^{2}=\lim _{t \rightarrow 0+} \sum_{k=1}^{\infty}\left|\frac{T_{t}\left(C\left(f_{k}\right)\right)-C\left(f_{k}\right)}{t}-\tilde{A}(C)\left(f_{k}\right)\right|_{H}^{2}=0,
$$

we have that

$$
\lim _{t \rightarrow 0+}\left|\frac{T_{t}\left(C\left(f_{k}\right)\right)-C\left(f_{k}\right)}{t}-\tilde{A}(C)\left(f_{k}\right)\right|_{H}^{2}=0
$$


for every $k \geq 1$. Therefore, $C\left(f_{k}\right) \in \mathcal{D}(A)$ and $\tilde{A}(C)\left(f_{k}\right)=A\left(C\left(f_{k}\right)\right)$ for each $k \geq 1$. Now pick any $f \in K$ and write

$$
f^{n}:=\sum_{k=1}^{n}<f, f_{k}>f_{k}, \quad n \geq 1 .
$$

Then $C\left(f^{n}\right)=\sum_{k=1}^{n}<f, f_{k}>C\left(f_{k}\right) \in \mathcal{D}(A), n \geq 1$, and $C(f)=\lim _{n \rightarrow \infty} C\left(f^{n}\right)$ in $H$. Now since $\tilde{A}(C) \in L_{2}(K, H) \subseteq L(K, H)$, it follows that

$$
\begin{aligned}
\tilde{A}(C)(f) & =\lim _{n \rightarrow \infty} \tilde{A}(C)\left(f^{n}\right) \\
& =\lim _{n \rightarrow \infty} \sum_{k=1}^{n}<f, f_{k}>\tilde{A}(C)\left(f_{k}\right) \\
& =\lim _{n \rightarrow \infty} \sum_{k=1}^{n}<f, f_{k}>A\left(C\left(f_{k}\right)\right) \\
& =\lim _{n \rightarrow \infty} A\left(C\left(f^{n}\right)\right) .
\end{aligned}
$$

Since $A$ is a closed operator, the above relation implies that $C(f) \in \mathcal{D}(A)$ and $A(C(f))=\tilde{A}(C)(f)$. As $\tilde{A}(C) \in L_{2}(K, H)$, and $f \in K$ is arbitrary, it follows that $C(K) \subseteq \mathcal{D}(A), A \circ C \in L_{2}(K, H)$ and $\tilde{A}(C)=A \circ C$. This proves (1.2.13) and completes the proof of the lemma.

Our main results in this section give regular versions $u: \mathbf{R}^{+} \times H \times \Omega \rightarrow H$ of mild solutions of (1.2.1) such that $u(t, \cdot, \omega) \in L(H)$ for all $(t, \omega) \in \mathbf{R}^{+} \times \Omega$ (Theorems 1.2.1-1.2.3). These regular versions are shown to be $L(H)$-valued cocycles with respect to the Brownian shift $\theta$ (Theorem 1.2.4). In order to formulate these regularity results, we will require the following lemma:

\section{Lemma 1.2.2.}

Let $B: H \rightarrow L_{2}(K, H)$ be continuous linear, and $v: \mathbf{R}^{+} \times \Omega \rightarrow L_{2}(H)$ be a $\left(\mathcal{B}\left(\mathbf{R}^{+}\right) \otimes\right.$ $\mathcal{F}, \mathcal{B}(H))$-measurable, $\left(\mathcal{F}_{t}\right)_{t \geq 0}$-adapted process such that $\int_{0}^{a} E\|v(t)\|_{L_{2}(H)}^{2} d t<\infty$ for each $a>0$. Then the random field $\int_{0}^{t} \tilde{T}_{t-s}(\{[B \circ v(s)](x)\}) d W(s), x \in H, t \geq 0$, admits a jointly measurable version which will be denoted by $\int_{0}^{t} T_{t-s} B v(s) d W(s)$ (by abuse of notation) and has the following properties:

(i) $\left[\int_{0}^{t} T_{t-s} B v(s) d W(s)\right](x)=\int_{0}^{t} \tilde{T}_{t-s}(\{[B \circ v(s)](x)\}) d W(s)$ for all $x \in H, t \geq 0$, a.s.

(ii) For a.a. $\omega \in \Omega$ and each $t \geq 0$, the map

$$
H \ni x \mapsto\left[\left(\int_{0}^{t} T_{t-s} B v(s) d W(s)\right)(\omega)\right](x) \in H
$$

is Hilbert-Schmidt.

Proof. 
To prove the lemma, we will define $\int_{0}^{t} T_{t-s} B v(s) d W(s)$ as an Itô stochastic integral with values in the Hilbert space $L_{2}(H)$ in the sense of [D-Z.1], Chapter 4). To do this, we will introduce the following notation.

For any $V \in L_{2}(H)$ and $B \in L\left(H, L_{2}(K, H)\right.$, define the linear map $B \star V: K \rightarrow L_{2}(H)$ by

$$
(B \star V)(f)(x):=B(V(x))(f), \quad f \in K, x \in H .
$$

Then $B \star V \in L_{2}\left(K, L_{2}(H)\right)$ because of the following computation

$$
\begin{aligned}
\|B \star V\|_{L_{2}\left(K, L_{2}(H)\right)}^{2} & =\sum_{k=1}^{\infty}\left\|(B \star V)\left(f_{k}\right)\right\|_{L_{2}(H)}^{2} \\
& =\sum_{k=1}^{\infty} \sum_{n=1}^{\infty}\left|(B \star V)\left(f_{k}\right)\left(e_{n}\right)\right|_{H}^{2} \\
& =\sum_{k=1}^{\infty} \sum_{n=1}^{\infty}\left|B\left(V\left(e_{n}\right)\right)\left(f_{k}\right)\right|^{2} \\
& =\sum_{n=1}^{\infty} \sum_{k=1}^{\infty}\left|B\left(V\left(e_{n}\right)\right)\left(f_{k}\right)\right|^{2} \\
& =\sum_{n=1}^{\infty}\left\|B\left(V\left(e_{n}\right)\right)\right\|_{L_{2}(K, H)}^{2} \\
& \leq\|B\|_{L\left(H, L_{2}(K, H)\right)}^{2}\|V\|_{L_{2}(H)}^{2}<\infty .
\end{aligned}
$$

Now let $v: \mathbf{R}^{+} \times \Omega \rightarrow L_{2}(H)$ be as in the lemma. Denote by $\tilde{\tilde{T}}_{t}: L_{2}\left(K, L_{2}(H)\right) \rightarrow L_{2}\left(K, L_{2}(H)\right)$ the induced lifting of $T_{t}: H \rightarrow H, t \geq 0$, via Lemma 1.2.1; i.e.

$$
\tilde{\tilde{T}}_{t}(C)(f):=T_{t} \circ C(f), \quad C \in L_{2}\left(K, L_{2}(H)\right), f \in K .
$$

Fix $t \in[0, a]$. Then the process $[0, t] \ni s \mapsto \tilde{\tilde{T}}_{t-s}(B \star v(s)) \in L_{2}\left(K, L_{2}(H)\right)$ is $\left(\mathcal{F}_{s}\right)_{0 \leq s \leq t}$-adapted and square-integrable, viz.

$$
\begin{aligned}
& E \int_{0}^{t}\left\|\tilde{\tilde{T}}_{t-s}(B \star v(s))\right\|_{L_{2}\left(K, L_{2}(H)\right)}^{2} d s \\
& \quad \leq\|B\|_{L\left(H, L_{2}(K, H)\right)}^{2} \sup _{0 \leq u \leq t}\left\|T_{u}\right\|_{L(H)}^{2} \int_{0}^{t} E\|v(s)\|_{L_{2}(H)}^{2} d s<\infty .
\end{aligned}
$$

In view of this, the $L_{2}(H)$-valued Itô stochastic integral $\int_{0}^{t} \tilde{\tilde{T}}_{t-s}(B \star v(s)) d W(s)$ is well-defined ([D-Z.1], Chapter 4). For simplicity of notation, we will denote this stochastic integral by

$$
\int_{0}^{t} T_{t-s} B v(s) d W(s):=\int_{0}^{t} \tilde{\tilde{T}}_{t-s}(B \star v(s)) d W(s) .
$$


This gives the required version of the the random field $\int_{0}^{t} \tilde{T}_{t-s}(\{[B \circ v(s)](x)\}) d W(s), x \in H, t \geq 0$, because

$$
\begin{aligned}
{\left[\int_{0}^{t} T_{t-s} B v(s) d W(s)\right] } & (x):=\left[\int_{0}^{t} \tilde{\tilde{T}}_{t-s}(B \star v(s)) d W(s)\right](x) \\
& =\sum_{k=1}^{\infty} \int_{0}^{t}\left[\tilde{\tilde{T}}_{t-s}(B \star v(s))\left(f_{k}\right)\right](x) d W^{k}(s) \\
& \left.=\sum_{k=1}^{\infty} \int_{0}^{t} T_{t-s}\{B \star v(s))\left(f_{k}\right)(x)\right\} d W^{k}(s) \\
& =\sum_{k=1}^{\infty} \int_{0}^{t} T_{t-s}\left\{B(v(s)(x))\left(f_{k}\right)\right\} d W^{k}(s) \\
& =\sum_{k=1}^{\infty} \int_{0}^{t} \tilde{T}_{t-s}\{[B \circ v(s)](x)\}\left(f_{k}\right) d W^{k}(s) \\
& =\int_{0}^{t} \tilde{T}_{t-s}\{[B \circ v(s)](x)\} d W(s)
\end{aligned}
$$

for all $x \in H$ and $t \geq 0$ a.s. In the above computation, we have used the fact that for fixed $x \in H$, the Itô stochastic integral commutes with the continuous linear evaluation map $L_{2}(H) \ni T \mapsto$ $T(x) \in H$.

\section{Theorem 1.2.1.}

Assume that for some $\alpha \in(0,1), A^{-\alpha}$ is trace-class, i.e., $\sum_{n=1}^{\infty} \mu_{n}^{-\alpha}<\infty$. Then the mild solution of the linear stochastic evolution equation (1.2.1) has a $\left(\mathcal{B}\left(\mathbf{R}^{+}\right) \otimes \mathcal{B}(H) \otimes \mathcal{F}, \mathcal{B}(H)\right)$-measurable version $u: \mathbf{R}^{+} \times H \times \Omega \rightarrow H$ with the following properties:

(i) For each $x \in H$, the process $u(\cdot, x, \cdot): \mathbf{R}^{+} \times \Omega \rightarrow H$ is $\left(\mathcal{B}\left(\mathbf{R}^{+}\right) \otimes \mathcal{F}, \mathcal{B}(H)\right)$-measurable, $\left(\mathcal{F}_{t}\right)_{t \geq 0}$-adapted and satisfies the stochastic integral equation (1.2.2).

(ii) For almost all $\omega \in \Omega$, the map $[0, \infty) \times H \ni(t, x) \rightarrow u(t, x, \omega) \in H$ is jointly continuous. Furthermore, for any fixed $a \in \mathbf{R}^{+}$,

$$
E \sup _{0 \leq t \leq a}\|u(t, \cdot, \cdot)\|_{L(H)}^{2 p}<\infty
$$

whenever $p \in\left(1, \alpha^{-1}\right]$.

(iii) For almost all $\omega \in \Omega$ and each $t>0, u(t, \cdot, \omega): H \rightarrow H$ is a Hilbert-Schmidt operator with the following representation:

$$
\begin{aligned}
u(t, \cdot, \cdot)=T_{t}+\sum_{n=1}^{\infty} \int_{0}^{t} T_{t-s_{1}} B & \int_{0}^{s_{1}} T_{s_{1}-s_{2}} B \cdots \\
& \quad \int_{0}^{s_{n-1}} T_{s_{n-1}-s_{n}} B T_{s_{n}} d W\left(s_{n}\right) \cdots d W\left(s_{2}\right) d W\left(s_{1}\right) .
\end{aligned}
$$


In the above equation, the iterated Itô stochastic integrals are interpreted in the sense of Lemma 1.2.2, and the convergence of the series holds in the Hilbert space $L_{2}(H)$ of HilbertSchmidt operators on $H$.

(iv) For almost all $\omega \in \Omega$, the path $[0, \infty) \ni t \mapsto u(t, \cdot, \omega)-T_{t} \in L_{2}(H)$ is continuous. In particular, the path $(0, \infty) \ni t \mapsto u(t, \cdot, \omega) \in L_{2}(H)$ is continuous for a.a. $\omega \in \Omega$. Furthermore, the process $u:(0, \infty) \times \Omega \rightarrow L_{2}(H)$ is $\left(\mathcal{F}_{t}\right)_{t \geq 0}$-adapted and $\left(\mathcal{B}((0, \infty)) \otimes \mathcal{F}, \mathcal{B}\left(L_{2}(H)\right)\right)$ measurable.

Proof.

Under the hypotheses on $A$, it is well known that the see $(1.2 .1)$ has a unique $\left(\mathcal{F}_{t}\right)_{t \geq 0^{-}}$ adapted mild solution $u$ satisfying the integral equation (1.2.2) in $H$. Moreover, (1.2.2) and a simple application of the Itô isometry together with Gronwall's lemma implies that

$$
\sup _{\substack{0 \leq t \leq a \\|x| \leq 1}} E\left[|u(t, x, \cdot)|^{2}\right]<\infty
$$

for each $a \in(0, \infty)$.

Applying (1.2.2) recursively, we obtain by induction

$$
\begin{aligned}
& u(t, x, \cdot) \\
& =T_{t} x+\sum_{k=1}^{n}\left[\int_{0}^{t} T_{t-s_{1}} B \int_{0}^{s_{1}} T_{s_{1}-s_{2}} B \cdots \int_{0}^{s_{k-1}} T_{s_{k-1}-s_{k}} B T_{s_{k}} d W\left(s_{k}\right) \cdots d W\left(s_{2}\right) d W\left(s_{1}\right)\right] x \\
& \quad+\int_{0}^{t} T_{t-s_{1}} B \int_{0}^{s_{1}} T_{s_{1}-s_{2}} B \cdots \int_{0}^{s_{n}} T_{s_{n}-s_{n+1}} B u\left(s_{n+1}, x, \cdot\right) d W\left(s_{n+1}\right) \cdots d W\left(s_{2}\right) d W\left(s_{1}\right) .
\end{aligned}
$$

Set $C_{t}:=\sup _{0 \leq s \leq t}\left\|T_{s} B\right\|_{L\left(H, L_{2}(K, H)\right)}^{2}$ for each $t>0$. Therefore,

$$
\begin{aligned}
& E\left[\left|\int_{0}^{t} T_{t-s_{1}} B \int_{0}^{s_{1}} T_{s_{1}-s_{2}} B \cdots \int_{0}^{s_{n}} T_{s_{n}-s_{n+1}} B u\left(s_{n+1}, x, \cdot\right) d W\left(s_{n+1}\right) \cdots d W\left(s_{2}\right) d W\left(s_{1}\right)\right|^{2}\right] \\
& =\int_{0}^{t} d s_{1} E\left[\left\|T_{t-s_{1}} B \int_{0}^{s_{1}} T_{s_{1}-s_{2}} B \cdots \int_{0}^{s_{n}} T_{s_{n}-s_{n+1}} B u\left(s_{n+1}, x, \cdot\right) d W\left(s_{n+1}\right) \cdots d W\left(s_{2}\right)\right\|_{L_{2}(K, H)}^{2}\right] \\
& \leq C_{t} \int_{0}^{t} d s_{1} E\left[\left|\int_{0}^{s_{1}} T_{s_{1}-s_{2}} B \cdots \int_{0}^{s_{n}} T_{s_{n}-s_{n+1}} B u\left(s_{n+1}, x, \cdot\right) d W\left(s_{n+1}\right) \cdots d W\left(s_{2}\right)\right|^{2}\right] \\
& \leq \cdots \cdots \\
& \leq C_{t}^{n} \int_{0}^{t} d s_{1} \int_{0}^{s_{1}} d s_{2} \cdots \int_{0}^{s_{n}} E\left[\left|u\left(s_{n+1}, x, \cdot\right)\right|^{2}\right] d s_{n+1} \leq C_{t}^{n} M \frac{t^{n}}{n !} \rightarrow 0 .
\end{aligned}
$$

This gives the following series representation of $u(t, x, \cdot)$ :

$$
\begin{aligned}
u(t, x, \cdot)=T_{t} x+\sum_{n=1}^{\infty} & {\left[\int_{0}^{t} T_{t-s_{1}} B \int_{0}^{s_{1}} T_{s_{1}-s_{2}} B \cdots \int_{0}^{s_{n-1}}\right.} \\
& \left.T_{s_{n-1}-s_{n}} B T_{s_{n}} d W\left(s_{n}\right) \cdots d W\left(s_{2}\right) d W\left(s_{1}\right)\right] x
\end{aligned}
$$


for each $x \in H$. The above series of iterated Itô stochastic integrals converges in $L^{2}(\Omega, H)$ uniformly in compacta in $t$ and for $x$ in bounded sets in $H$.

Using the fact that $A^{-1}$ is trace class, we will show further that the series expansion (1.2.18) actually holds in the Hilbert space $L^{2}\left(\Omega, L_{2}(H)\right)$. To see this, first observe that $T_{t}$ and all the terms in the series on the right hand side of (1.2.18) are Hilbert-Schmidt for any fixed $t>0$. We use the comparison test to conclude that the series on the right hand side of (1.2.18) converges (absolutely) in $L^{2}\left(\Omega, L_{2}(H)\right)$. Fix $a>0$. Then by successive applications of the Itô isometry (in $L_{2}(H)$ ), one gets

$$
\begin{aligned}
E & \left\|\int_{0}^{a} T_{a-s_{1}} B \int_{0}^{s_{1}} T_{s_{1}-s_{2}} B \cdots \int_{0}^{s_{n}} T_{s_{n}-s_{n+1}} B T_{s_{n+1}} d W\left(s_{n+1}\right) \cdots d W\left(s_{2}\right) d W\left(s_{1}\right)\right\|_{L_{2}(H)}^{2} \\
& =\int_{0}^{a} d s_{1} E\left[\| T_{a-s_{1}} B \int_{0}^{s_{1}} T_{s_{1}-s_{2}} B \cdots \int_{0}^{s_{n}}\right. \\
& \left.\leq T_{s_{n}-s_{n+1}} B T_{s_{n+1}} d W\left(s_{n+1}\right) \cdots d W\left(s_{2}\right) \|_{L_{2}\left(K, L_{2}(H)\right)}^{2}\right] \\
& \leq \cdots \\
& \leq C_{a}^{n} \int_{0}^{a} d s_{1} E\left[\left\|\int_{0}^{s_{1}} T_{s_{1}-s_{2}} B \cdots \int_{0}^{s_{n}} T_{s_{n}-s_{n+1}}^{s_{1}} d T_{s_{n+1}} d W\left(s_{n+1}\right) \cdots d W\left(s_{2}\right)\right\|_{L_{2}(H)}^{2} E\right. \\
& \leq C_{a}^{n} \frac{a^{n}}{n !} \int_{0}^{s_{n}}\left\|T_{s}\right\|_{L_{2}(H)}^{2} d s=C_{a}^{n} \frac{a^{n}}{n !} \int_{0}^{a} \sum_{k=1}^{\infty} e^{-2 \mu_{k} s} d s \\
& \leq C_{a}^{n} \frac{a^{n}}{n !} \sum_{k=1}^{\infty} \frac{1}{2 \mu_{k}}
\end{aligned}
$$

for each integer $n \geq 1$. This implies that the expansion (1.2.18) converges in $L^{2}\left(\Omega, L_{2}(H)\right)$ for each $t>0$. Hence assertion (iii) of the theorem holds.

We next prove assertion (iv). Consider the series in (1.2.18) and let $\Phi^{n}(t) \in L_{2}(H)$ be its general term, viz.

$$
\Phi^{n}(t):=\int_{0}^{t} T_{t-s_{1}} B \int_{0}^{s_{1}} T_{s_{1}-s_{2}} B \cdots \int_{0}^{s_{n-1}} T_{s_{n-1}-s_{n}} B T_{s_{n}} d W\left(s_{n}\right) \cdots d W\left(s_{2}\right) d W\left(s_{1}\right)
$$

for $t \geq 0, n \geq 1$. Note the relations

$$
\left.\begin{array}{l}
\Phi^{n}(t)=\int_{0}^{t} T_{t-s_{1}} B \Phi^{n-1}\left(s_{1}\right) d W\left(s_{1}\right), \quad n \geq 2, \\
\Phi^{1}(t)=\int_{0}^{t} T_{t-s_{1}} B T_{s_{1}} d W\left(s_{1}\right),
\end{array}\right\}
$$

for $t \geq 0$. 
First, we show by induction that for each $n \geq 1$, the process $\Phi^{n}:[0, \infty) \times \Omega \rightarrow L_{2}(H)$ has a version with a.a. sample paths continuous on $[0, \infty)$. In view of $(1.2 .21)$, this will follow from Proposition (7.3) ([D-Z.1], p. 184) provided we show that

$$
\int_{0}^{a} E\left\|\Phi^{n-1}(t)\right\|_{L_{2}(H)}^{2 p} d t<\infty
$$

for all integers $n>1$ and $p \in\left(1, \alpha^{-1}\right]$. For later use, we will actually prove the stronger estimate

$$
E \sup _{0 \leq s \leq t}\left\|\Phi^{n}(s)\right\|_{L_{2}(H)}^{2 p} \leq K_{1} \frac{\left(K_{2} t\right)^{n-1}}{(n-1) !}, \quad t \in[0, a],
$$

for all integers $n \geq 1$, and $p \in\left(1, \alpha^{-1}\right]$, where $K_{1}, K_{2}$ are positive constants depending only on $p$ and $a$. We use induction on $n$ to establish $\left(1.2 .22^{\prime}\right)$. To check $\left(1.2 .22^{\prime}\right)$ for $n=1$, choose $p \in\left(1, \alpha^{-1}\right]$, and consider the following easy estimates:

$$
\begin{aligned}
\left\{\int_{0}^{a}\left\|T_{s}\right\|_{L_{2}(H)}^{2 p} d s\right\}^{1 / p} & =\left\{\int_{0}^{a}\left[\sum_{k=1}^{\infty} e^{-2 \mu_{k} s}\right]^{p} d s\right\}^{1 / p} \\
& \leq \sum_{k=1}^{\infty}\left\{\int_{0}^{a} e^{-2 \mu_{k} p s} d s\right\}^{1 / p} \\
& \leq \frac{1}{(2 p)^{1 / p}} \sum_{k=1}^{\infty} \mu_{k}^{-1 / p} \\
& \leq \frac{1}{(2 p)^{1 / p}} \sum_{k=1}^{\infty} \mu_{k}^{-\alpha}<\infty .
\end{aligned}
$$

Now use the second equality in (1.2.21) and Proposition (7.3) ([D-Z.1], p. 184) to get the following estimate:

$$
E \sup _{0 \leq s \leq t}\left\|\Phi^{1}(s)\right\|_{L_{2}(H)}^{2 p} \leq C_{1} \int_{0}^{t}\left\|T_{s_{1}}\right\|_{L_{2}(H)}^{2 p} d s_{1} \leq \frac{C_{1}}{2 p}\left[\sum_{k=1}^{\infty} \mu_{k}^{-\alpha}\right]^{p}
$$

for all $t \in[0, a]$ and for $p \in\left(1, \alpha^{-1}\right]$. The constant $C_{1}$ does not depend on $t \in[0, a]$. Since $A^{-\alpha}$ is trace-class, the above inequality implies that $\left(1.2 .22^{\prime}\right)$ holds with $K_{1}:=\frac{C_{1}}{2 p}\left[\sum_{k=1}^{\infty} \mu_{k}^{-\alpha}\right]^{p}$, for $n=1$, and any $p \in\left(1, \alpha^{-1}\right]$. Now suppose that $\left(1.2 .22^{\prime}\right)$ holds for some integer $n \geq 1$ and all $p \in\left(1, \alpha^{-1}\right]$. Then the first equality in (1.2.21) and Proposition (7.3) ([D-Z.1], p. 184) imply that there is a positive constant $K_{2}:=K_{2}(p, a)$ such that

$$
\begin{aligned}
E \sup _{0 \leq s \leq t}\left\|\Phi^{n+1}(s)\right\|_{L_{2}(H)}^{2 p} & \leq K_{2} \int_{0}^{t} E\left\|\Phi^{n}\left(s_{1}\right)\right\|_{L_{2}(H)}^{2 p} d s_{1} \\
& \leq K_{2} \int_{0}^{t} K_{1} \frac{\left(K_{2} s_{1}\right)^{n-1}}{(n-1) !} d s_{1}=K_{1} \frac{\left(K_{2} t\right)^{n}}{n !}
\end{aligned}
$$

for all $t \in[0, a]$ and $p \in\left(1, \alpha^{-1}\right]$. Therefore by induction, (1.2.22') (and hence (1.2.22)) hold for all integers $n \geq 1$ and any $p \in\left(1, \alpha^{-1}\right]$. 
From the first equality in (1.2.21), (1.2.22) and Proposition 7.3 ([D-Z.1], p. 184), it follows that each $\Phi^{n}:[0, \infty) \times \Omega \rightarrow L_{2}(H)$ has a version with a.a. sample paths continuous on $[0, \infty)$. From the estimate $\left(1.2 .22^{\prime}\right)$, it is easy to see that the series $\sum_{n=1}^{\infty} \Phi^{n}$ converges absolutely in $L^{2 p}\left(\Omega, C\left([0, a], L_{2}(H)\right)\right)$ for each $a>0$ and $p \in\left(1, \alpha^{-1}\right]$. This gives a continuous modification for the sum $\sum_{n=1}^{\infty} \Phi^{n}$ of the series in (1.2.18). Hence the $L_{2}(H)$-valued process

$$
u(t, \cdot, \cdot)-T_{t}=\sum_{n=1}^{\infty} \Phi^{n}(t), \quad t \geq 0,
$$

has a version with almost all sample-paths continuous on $[0, \infty)$. This proves the first assertion in (iv). To prove the second assertion in (iv), it suffices to show that the mapping $(0, \infty) \ni t \mapsto T_{t} \in$ $L_{2}(H)$ is locally Lipschitz. To see this, let $0<t_{1}<t_{2} \leq a<\infty$. Then

$$
\begin{aligned}
\left\|T_{t_{2}}-T_{t_{1}}\right\|_{L_{2}(H)}^{2} & \leq \sum_{k=1}^{\infty}\left[e^{-\mu_{k} t_{2}}-e^{-\mu_{k} t_{1}}\right]^{2} \\
& \leq\left(t_{2}-t_{1}\right)^{2} \sum_{k=1}^{\infty} \mu_{k}^{2} e^{-2 \mu_{k} t_{1}} \\
& \leq \frac{3}{4 t_{1}^{3}}\left(t_{2}-t_{1}\right)^{2} \sum_{k=1}^{\infty} \mu_{k}^{-1} .
\end{aligned}
$$

Since $A^{-1}$ is trace-class, the above inequality implies that the mapping $(0, \infty) \ni t \mapsto T_{t} \in L_{2}(H)$ is locally Lipschitz. The second assertion in (iv) now follows immediately from this and the first assertion.

The measurability assertions in (iv) follow directly from the relation

$$
u(t, \cdot, \cdot)=T_{t}+\sum_{n=1}^{\infty} \Phi^{n}(t), \quad t>0
$$

and the fact that, as $L_{2}(H)$-valued Itô stochastic integrals, the processes $\Phi^{n}:(0, \infty) \times \Omega \rightarrow$ $L_{2}(H), n \geq 1$, are $\left(\mathcal{F}_{t}\right)_{t>0}$-adapted and $\left(\mathcal{B}((0, \infty)) \otimes \mathcal{F}, \mathcal{B}\left(L_{2}(H)\right)\right)$-measurable.

The evaluation map

$$
\begin{gathered}
L_{2}(H) \times H \rightarrow H \\
(S, x) \mapsto S(x)
\end{gathered}
$$

is continuous bilinear. Therefore the first assertion in (iv) implies that the map $[0, T] \times H \ni(t, x) \rightarrow$ $u(t, x, \omega)-T_{t}(x) \in H$ is jointly continuous for almost all $\omega \in \Omega$. Since $[0, T] \times H \ni(t, x) \rightarrow T_{t}(x) \in$ $H$ is jointly continuous (by strong continuity of the semigroup $T_{t}, t \geq 0$ ), the first assertion in (ii) follows. 
Finally, it remains to prove the estimate in (ii). In view of $\left(1.2 .22^{\prime}\right)$, the series in $(1.2 .18)$ converges absolutely in $L^{2 p}\left(\Omega, C\left([0, a], L_{2}(H)\right)\right), p \in\left(1, \alpha^{-1}\right]$. Therefore,

$$
\begin{aligned}
\left\{E \sup _{0 \leq t \leq a}\|u(t, \cdot, \cdot)\|_{L(H)}^{2 p}\right\}^{1 /(2 p)} & \leq \sup _{0 \leq t \leq a}\left\|T_{t}\right\|_{L(H)}+\sum_{n=1}^{\infty}\left\{E \sup _{0 \leq t \leq a}\left\|\Phi^{n}(t)\right\|_{L_{2}(H)}^{2 p}\right\}^{1 /(2 p)} \\
& \leq \sup _{0 \leq t \leq a}\left\|T_{t}\right\|_{L(H)}+K_{1}^{1 /(2 p)} \sum_{k=1}^{\infty}\left\{\frac{\left(K_{2} a\right)^{n-1}}{(n-1) !}\right\}^{1 /(2 p)}<\infty .
\end{aligned}
$$

This proves the estimate in (ii), and the proof of Theorem 1.2.1 is complete.

\section{Theorem 1.2.2.}

Assume the following:

(i) $A^{-1}$ is a trace class operator, i.e., $\sum_{n=1}^{\infty} \mu_{n}^{-1}<\infty$.

(ii) $T_{t} \in L(H), t \geq 0$, is a strongly continuous contraction semigroup.

Then the mild solution of the linear stochastic evolution equation (1.2.1) has a version $u: \mathbf{R}^{+} \times H \times \Omega \rightarrow H$ which satisfies the assertions (i), (iii) and (iv) of Theorem 1.2.1. Furthermore, for almost all $\omega \in \Omega$, the map $[0, \infty) \times H \ni(t, x) \rightarrow u(t, x, \omega) \in H$ is jointly continuous, and for any fixed $a \in \mathbf{R}^{+}$,

$$
E \sup _{0 \leq t \leq a}\|u(t, \cdot, \cdot)\|_{L(H)}^{2}<\infty
$$

Proof.

The proof follows that of Theorem 1.2.1. We will only highlight the differences.

We assume Hypotheses (i) and (ii). By the proof of Theorem 1.2.1, Hypothesis (i) implies that the solution of (1.2.1) admits a version $u: \mathbf{R}^{+} \times H \times \Omega \rightarrow H$ which satisfies assertions (i) and (iii) of Theorem 1.2.1.

Use the notation in the proof of Theorem 1.2.1. In particular, one has

$$
u(t, \cdot, \cdot)-T_{t}=\sum_{n=1}^{\infty} \Phi^{n}(t), \quad t \geq 0,
$$

where the series converges in $L^{2}\left(\Omega, L_{2}(H)\right)$ for each $t \geq 0$. Since $A^{-1}$ is trace-class, then $\int_{0}^{a}\left\|T_{s}\right\|_{L_{2}(H)}^{2} d s<\infty$. Using this, the fact that $T_{t}, t \geq 0$, is a contraction semigroup, and Theorem 6.10 ([D-Z.1], p. 160), it follows that $\Phi^{1}:[0, \infty) \times \Omega \rightarrow L_{2}(H)$ has a sample-continuous version. Furthermore, there is a positive constant $K_{3}$ such that

$$
E \sup _{0 \leq s \leq t}\left\|\Phi^{1}(s)\right\|_{L_{2}(H)}^{2} \leq K_{3} \int_{0}^{t}\left\|T_{s}\right\|_{L_{2}(H)}^{2} d s<\infty
$$


for all $t \in[0, a]$ ([D-Z.1], Theorem 6.10, p. 160). We will show that the series $\sum_{n=2}^{\infty} \Phi^{n}$ converges in $L^{2 p}\left(\Omega, C\left([0, a], L_{2}(H)\right)\right.$ for all $p \geq 1$. Therefore, the series $\sum_{n=1}^{\infty} \Phi^{n}$ converges in $L^{2}\left(\Omega, C\left([0, a], L_{2}(H)\right)\right)$.

By Lemma (7.2), ([D-Z.1], p. 182), we have

$$
E\left\|\Phi^{1}(t)\right\|_{L_{2}(H)}^{2 p} \leq K_{4}\left[\int_{0}^{t}\left\|T_{s}\right\|_{L_{2}(H)}^{2} d s\right]^{p}
$$

for all $t \in[0, a]$ and all $p \geq 1$. The constant $K_{4}$ depends on $p$ but is independent of $t \in[0, a]$. Since $A^{-1}$ is trace-class, the above inequality, Proposition 7.3 ([D-Z.1], p. 184) and an induction argument imply the following inequality:

$$
E \sup _{0 \leq s \leq t}\left\|\Phi^{n}(s)\right\|_{L_{2}(H)}^{2 p} \leq K_{5} \frac{\left(K_{6} t\right)^{n-1}}{(n-1) !}, \quad t \in[0, a]
$$

for all integers $n \geq 2$, and $p \geq 1$, where $K_{5}, K_{6}$ are positive constants depending only on $p$ and $a$ (cf. (1.2.22') in the proof of Theorem 1.2.1). The rest of the proof of the theorem follows from the above inequality by a similar argument to the one in the proof of Theorem 1.2.1.

\section{Theorem 1.2.3.}

Assume that $\sum_{n=1}^{\infty} \mu_{n}^{-1}\left\|B\left(e_{n}\right)\right\|_{L_{2}(K, H)}^{2}<\infty$.

Then the mild solution of the linear stochastic evolution equation $(1.2 .1)$ has a $\left(\mathcal{B}\left(\mathbf{R}^{+}\right) \otimes\right.$ $\mathcal{B}(H) \otimes \mathcal{F}, \mathcal{B}(H))$-measurable version $u: \mathbf{R}^{+} \times H \times \Omega \rightarrow H$ with the following properties:

(i) For each $x \in H$, the process $u(\cdot, x, \cdot): \mathbf{R}^{+} \times \Omega \rightarrow H$ is $\left(\mathcal{B}\left(\mathbf{R}^{+}\right) \otimes \mathcal{F}, \mathcal{B}(H)\right)$-measurable, $\left(\mathcal{F}_{t}\right)_{t \geq 0}$-adapted and satisfies the stochastic integral equation (1.2.2).

(ii) For almost all $\omega \in \Omega$, the map $[0, \infty) \times H \ni(t, x) \rightarrow u(t, x, \omega) \in H$ is jointly continuous. Furthermore, for any fixed $a \in \mathbf{R}^{+}$,

$$
E \sup _{0 \leq t \leq a}\|u(t, \cdot, \cdot)\|_{L(H)}^{2}<\infty .
$$

(iii) For almost all $\omega \in \Omega$ and each $t>0, u(t, \cdot, \omega): H \rightarrow H$ is a bounded linear operator with the following representation:

$$
\begin{gathered}
u(t, \cdot, \cdot)=T_{t}+\sum_{n=1}^{\infty} \int_{0}^{t} T_{t-s_{1}} B \int_{0}^{s_{1}} T_{s_{1}-s_{2}} B \cdots \\
\cdots \int_{0}^{s_{n-1}} T_{s_{n-1}-s_{n}} B T_{s_{n}} d W\left(s_{n}\right) \cdots d W\left(s_{2}\right) d W\left(s_{1}\right) .
\end{gathered}
$$

In the above equation, the iterated Itô stochastic integrals are interpreted in the sense of Lemma 1.2.2, and the convergence of the series holds in the Hilbert space $L_{2}(H)$ of HilbertSchmidt operators on $H$. If in addition, $T_{t}: H \rightarrow H$ is compact for each $t>0$, then so is $u(t, \cdot, \omega): H \rightarrow H$ for almost all $\omega \in \Omega$. 
(iv) For almost all $\omega \in \Omega$, the path $[0, \infty) \ni t \mapsto u(t, \cdot, \omega)-T_{t} \in L_{2}(H)$ is continuous. Furthermore, the process $u:(0, \infty) \times \Omega \rightarrow L(H)$ is $\left(\mathcal{F}_{t}\right)_{t \geq 0}$-adapted and $(\mathcal{B}((0, \infty)) \otimes \mathcal{F}, \mathcal{B}(L(H)))$ measurable.

Proof.

The proof follows along the same lines as that of Theorem 1.2.1. Just observe that the hypothesis of Theorem 1.2.3 implies the following integrability property

$$
\int_{0}^{a}\left\|B T_{t}\right\|_{L_{2}\left(H, L_{2}(K, H)\right)}^{2} d t<\infty
$$

for any $a>0$.

Remark.

It is easy to see that the hypothesis of Theorem 1.2.3 is satisfied if one assumes that the mapping $B: H \rightarrow L_{2}(K, H)$ is Hilbert-Schmidt. By contrast to the hypotheses of Theorems 1.2.1, 1.2.2, the assumption in Theorem 1.2.3 does not entail any dimension restriction if the operator $A$ is a differential operator on a Euclidean domain. Furthermore, one does not require even discreteness of the spectrum of $A$ if we assume that $B: H \rightarrow L_{2}(K, H)$ is Hilbert-Schmidt. However, in this case, one gets a flow of bounded linear (but not necessarily compact) maps $u(t, \cdot, \omega) \in L(H), t>0, \omega \in \Omega$.

We will continue to assume the hypotheses of Theorem 1.2.1, 1.2.2 or 1.2.3.

Let $u: \mathbf{R}^{+} \times \Omega \rightarrow L(H)$ be the regular version of the mild solution of (1.2.1) given by Theorems 1.2.1, 1.2.2 or 1.2.3. Our next result in this section identifies $u$ as a fundamental solution (or parametrix) for (1.2.1).

Consider the following stochastic integral equation:

$$
\left.\begin{array}{l}
v(t)=T_{t}+\int_{0}^{t} T_{t-s} B v(s) d W(s), \quad t>0 \\
v(0)=I,
\end{array}\right\}
$$

where $I$ denotes the identity operator on $H$ and the stochastic integral is interpreted as an Itô integral in the Hilbert space $L_{2}(H)$.

Remark.

The initial-value problem (1.2.27) cannot be interpreted strictly in the Hilbert space $L_{2}(H)$ since $v(0)=I \notin L_{2}(H)$. On the other hand, one cannot view the equation (1.2.27) in the Banach space $L(H)$, because the latter Banach space is not sufficiently "smooth" to allow for a satisfactory theory of stochastic integration.

We say that a stochastic process $v:[0, \infty) \times \Omega \rightarrow L(H)$ is a solution to equation (1.2.27) if (i) $v:(0, \infty) \times \Omega \rightarrow L_{2}(H)$ is $\left(\mathcal{F}_{t}\right)_{t>0}$-adapted, and $\left(\mathcal{B}((0, \infty)) \otimes \mathcal{F}, \mathcal{B}\left(L_{2}(H)\right)\right)$-measurable. 
(ii) $v \in L^{2}\left((0, a) \times \Omega, L_{2}(H)\right)$ for all $a \in(0, \infty)$.

(iii) $v$ satisfies (1.2.27) almost surely.

\section{Theorem 1.2.3'}

Assume the hypotheses of Theorems 1.2.1, 1.2.2. Let $u$ be the regular version of the mild solution of (1.2.1) given therein. Then $u$ is the unique solution of $(1.2 .27)$ in $L^{2}\left((0, a) \times \Omega, L_{2}(H)\right)$ for $a>0$.

Proof.

Assume the hypotheses of Theorems 1.2.1, 1.2.2 or 1.2.3. Let $u$ be the regular version of the mild solution of (1.2.1) given by these theorems.

Note first that $u:(0, \infty) \times \Omega \rightarrow L_{2}(H)$ is $\left(\mathcal{F}_{t}\right)_{t \geq 0}$-adapted, and $\left(\mathcal{B}((0, \infty)) \otimes \mathcal{F}, \mathcal{B}\left(L_{2}(H)\right)\right)$ measurable. This follows from assertion (iv) in Theorem 1.2.1.

In the proofs of Theorems 1.2.1, 1.2.2, we have shown that the series $\sum_{n=1}^{\infty} \Phi^{n}$ converges absolutely in $L^{2}\left(\Omega, C\left([0, a], L_{2}(H)\right)\right)$, and hence also in $L^{2}\left(\Omega, L^{2}\left((0, a), L_{2}(H)\right)\right)$, because of the continuous linear embedding

$$
L^{2}\left(\Omega, C\left([0, a], L_{2}(H)\right)\right) \subset L^{2}\left(\Omega, L^{2}\left((0, a), L_{2}(H)\right)\right) \equiv L^{2}\left((0, a) \times \Omega, L_{2}(H)\right) .
$$

Thus

$$
\int_{0}^{a} E\|u(t, \cdot, \cdot)\|_{L_{2}(H)}^{2} d t \leq 2 \int_{0}^{a}\left\|T_{t}\right\|_{L_{2}(H)}^{2} d t+2\left[\sum_{n=1}^{\infty}\left\{\int_{0}^{a} E\left\|\Phi^{n}(t)\right\|_{L_{2}(H)}^{2} d t\right\}^{1 / 2}\right]^{2}<\infty .
$$

In particular, the Itô stochastic integral $\int_{0}^{t} T_{t-s} B u(s) d W(s)$ is well-defined in $L_{2}(H)$ for each $t \in[0, a]$ (Lemma 1.2.2).

We next show that $u$ solves the operator-valued stochastic integral equation (1.2.27). To see this, use the fact that

$$
\left[\int_{0}^{t} T_{t-s} B u(s, \cdot, \cdot) d W(s)\right]\left(e_{n}\right)=\int_{0}^{t} T_{t-s} B u\left(s, e_{n}, \cdot\right) d W(s), \quad n \geq 1
$$

and the integral equation (1.2.2) to conclude that

$$
u(t, \omega)\left(e_{n}\right)=T_{t} e_{n}+\left[(\omega) \int_{0}^{t} T_{t-s} B u(s, \cdot, \cdot) d W(s)\right]\left(e_{n}\right), \quad t \geq 0, n \geq 1,
$$

holds for all $\omega$ in a sure event $\Omega^{*} \in \mathcal{F}$ which is independent of $n$ and $t \geq 0$. Since $\left\{e_{n}: n \geq 1\right\}$ is a complete orthonormal system in $H$, it follows from (1.2.28) that for all $\omega \in \Omega^{*}$, one has

$$
u(t, \omega)(x)=T_{t} x+(\omega) \int_{0}^{t}\left[T_{t-s} B u(s, \cdot, \cdot) d W(s)\right](x), \quad t \geq 0, n \geq 1
$$


for all $x \in H$. Thus $u$ is a solution of (1.2.27).

Finally we show that (1.2.27) has a unique $\left(\mathcal{F}_{t}\right)_{t>0}$-adapted solution in $L^{2}\left((0, a) \times \Omega, L_{2}(H)\right)$. Suppose $v_{1}, v_{2}$ are two such solutions of (1.2.27). Then

$$
E\left\|v_{1}(t)-v_{2}(t)\right\|_{L_{2}(H)}^{2} \leq\|B\|_{L_{2}(K, H)} \sup _{0 \leq u \leq a}\left\|T_{u}\right\|_{L(H)} \int_{0}^{t} E\left\|v_{1}(s)-v_{2}(s)\right\|_{L_{2}(H)}^{2} d s
$$

for all $t \in(0, a]$. The above inequality implies that $E\left\|v_{1}(t)-v_{2}(t)\right\|_{L_{2}(H)}^{2}=0$ for all $t>0$ and uniqueness holds.

From now on and throughout this section, we will impose the following Condition (B):

(i) The operator $B: H \rightarrow L_{2}(K, H)$ can be extended to a bounded linear operator $H \rightarrow$ $L(E, H)$, which will also be denoted by $B$.

(ii) The series $\sum_{k=1}^{\infty}\left\|B_{k}^{2}\right\|_{L(H)}$ converges, where the bounded linear operators $B_{k}: H \rightarrow H$ are defined by $B_{k}(x):=B(x)\left(f_{k}\right), x \in H, k \geq 1$.

\section{Theorem 1.2.4.}

Suppose the hypotheses of Theorems 1.2.1, 1.2.2 or 1.2.3, and Condition (B) are satisfied. Then the mild solution of (1.2.1) admits a version $u: \mathbf{R}^{+} \times \Omega \rightarrow L(H)$ satisfying Theorems 1.2.1 or 1.2 .2 and is such that

(i) $(u, \theta)$ is a perfect $L(H)$-valued cocycle:

$$
u(t+s, \omega)=u(t, \theta(s, \omega)) \circ u(s, \omega)
$$

for all $s, t \geq 0$ and all $\omega \in \Omega$;

(ii) $\sup _{0 \leq s \leq t \leq a}\|u(t-s, \theta(s, \omega))\|_{L(H)}<\infty$, for all $\omega \in \Omega$ and all $a>0$.

Proof.

In view of Theorem $1.2 .3^{\prime}, u$ satisfies the stochastic integral equation

$$
\left.\begin{array}{l}
u(t)=T_{t}+\int_{0}^{t} T_{t-s} B u(s) d W(s), \quad t>0 \\
u(0)=I
\end{array}\right\}
$$

with $u(t) \in L_{2}(H)$ a.s. for all $t>0$.

Our strategy for proving the cocycle property (1.2.31) is to approximate the cylindrical Wiener process $W$ in (1.2.32) by a suitably defined family of smooth processes $W_{n}: \mathbf{R}^{+} \times \Omega \rightarrow$ $E, n \geq 1$, prove the cocycle property for the corresponding approximating solutions and then pass to the limit in $L_{2}(H)$ as $n$ tends to $\infty$. 
Define $W_{n}$ on $\mathbf{R}^{+} \times \Omega, n \geq 1$, by

$$
W_{n}(t, \omega):=n \int_{t-1 / n}^{t} W(u, \omega) d u-n \int_{-1 / n}^{0} W(u, \omega) d u, \quad t \geq 0, \omega \in \Omega .
$$

It is easy to see that each $W_{n}$ is a helix:

$$
W_{n}\left(t, \theta\left(t_{1}, \omega\right)\right)=W_{n}\left(t+t_{1}, \omega\right)-W_{n}\left(t_{1}, \omega\right)
$$

and

$$
W_{n}^{\prime}\left(t, \theta\left(t_{1}, \omega\right)\right)=W_{n}^{\prime}\left(t+t_{1}, \omega\right)
$$

for all $t, t_{1} \geq 0, \omega \in \Omega, n \geq 1$. In (1.2.35), the prime ' denotes differentiation with respect to $t$.

For each $k \geq 1$, recall the definition of $B_{k}: H \rightarrow H$ in Condition (B)(ii). For each integer $n \geq 1$, define the process $u_{n}: \mathbf{R}^{+} \times \Omega \rightarrow L_{2}(H)$ to be the unique $\left(\mathcal{B}((0, \infty)) \otimes \mathcal{F}, \mathcal{B}\left(L_{2}(H)\right)\right)$ measurable, $\left(\mathcal{F}_{t}\right)_{t>0}$-adapted solution of the random integral equation:

$$
\begin{aligned}
& u_{n}(t, \omega)=T_{t}+\int_{0}^{t} T_{t-s} \circ\left\{\left[B \star u_{n}(s, \omega)\right]\left(W_{n}^{\prime}(s, \omega)\right)\right\} d s \\
& \quad-\frac{1}{2} \int_{0}^{t} \sum_{k=1}^{\infty} T_{t-s} \circ B_{k}^{2} \circ u_{n}(s, \omega) d s, \quad t>0 \\
& u_{n}(0, \omega)=I,
\end{aligned}
$$

for $\omega \in \Omega$. Recall that the operation $\star$ is defined by (1.2.16) in the proof of Lemma 1.2.2.

Then

$$
\lim _{n \rightarrow \infty} \sup _{0<t \leq a}\left\|u_{n}(t)-u(t)\right\|_{L_{2}(H)}^{2}=0,
$$

in probability, for each $a>0$. The convergence (1.2.36) follows by modifying the proof (in $L_{2}(H)$ ) of the Wong-Zakai approximation theorem for stochastic evolution equations in ([Tw], Theorem 1.3.4.1). (Cf. [I-W], Theorem 7.2, p. 497).

Next, we show that for each $n \geq 1,\left(u_{n}, \theta\right)$ is a perfect cocycle. Fix $n \geq 1, t_{1} \geq 0$ and $\omega \in \Omega$. Using $(1.2 .27)(n)$, it follows that

$$
\begin{aligned}
u_{n}( & \left.t, \theta\left(t_{1}, \omega\right)\right) \circ u_{n}\left(t_{1}, \omega\right) \\
= & T_{t} \circ u_{n}\left(t_{1}, \omega\right) \\
& +\int_{t_{1}}^{t+t_{1}} T_{t+t_{1}-s} \circ\left\{\left[B \star\left(u_{n}\left(s-t_{1}, \theta\left(t_{1}, \omega\right)\right) \circ u_{n}\left(t_{1}, \omega\right)\right)\right]\left(W_{n}^{\prime}\left(s-t_{1}, \theta\left(t_{1}, \omega\right)\right)\right)\right\} d s \\
& -\frac{1}{2} \int_{t_{1}}^{t+t_{1}} \sum_{k=1}^{\infty} T_{t+t_{1}-s} \circ B_{k}^{2} \circ\left(u_{n}\left(s-t_{1}, \theta\left(t_{1}, \omega\right)\right) \circ u_{n}\left(t_{1}, \omega\right)\right) d s \\
= & T_{t+t_{1}}+\int_{0}^{t_{1}} T_{t+t_{1}-s} \circ\left\{\left[B \star u_{n}(s, \omega)\right]\left(W_{n}^{\prime}(s, \omega)\right)\right\} d s \\
& -\frac{1}{2} \int_{0}^{t_{1}} \sum_{k=1}^{\infty} T_{t+t_{1}-s} \circ B_{k}^{2} \circ u_{n}(s, \omega) d s
\end{aligned}
$$




$$
\begin{aligned}
& +\int_{t_{1}}^{t+t_{1}} T_{t+t_{1}-s} \circ\left\{\left[B \star\left(u_{n}\left(s-t_{1}, \theta\left(t_{1}, \omega\right)\right) \circ u_{n}\left(t_{1}, \omega\right)\right)\right]\left(W_{n}^{\prime}\left(s-t_{1}, \theta\left(t_{1}, \omega\right)\right)\right)\right\} d s \\
& -\frac{1}{2} \int_{t_{1}}^{t+t_{1}} \sum_{k=1}^{\infty} T_{t+t_{1}-s} \circ B_{k}^{2} \circ\left(u_{n}\left(s-t_{1}, \theta\left(t_{1}, \omega\right)\right) \circ u_{n}\left(t_{1}, \omega\right)\right) d s, \quad t>0 .
\end{aligned}
$$

Hence, using $(1.2 .27)(n)$ and (1.2.35), we obtain

$$
\begin{aligned}
& u_{n}\left(t, \theta\left(t_{1}, \omega\right)\right) \circ u_{n}\left(t_{1}, \omega\right)-u_{n}\left(t_{1}+t, \omega\right) \\
& =\int_{t_{1}}^{t+t_{1}} T_{t+t_{1}-s} \circ\left\{\left[B \star\left(u_{n}\left(s-t_{1}, \theta\left(t_{1}, \omega\right)\right) \circ u_{n}\left(t_{1}, \omega\right)-u_{n}(s, \omega)\right)\right]\right. \\
& \left.\quad\left(W_{n}^{\prime}\left(s-t_{1}, \theta\left(t_{1}, \omega\right)\right)\right)\right\} d s \\
& \quad-\frac{1}{2} \int_{t_{1}}^{t+t_{1}} \sum_{k=1}^{\infty} T_{t+t_{1}-s} \circ B_{k}^{2} \circ\left[u_{n}\left(s-t_{1}, \theta\left(t_{1}, \omega\right)\right) \circ u_{n}\left(t_{1}, \omega\right)-u_{n}(s, \omega)\right] d s \\
& =\int_{0}^{t} T_{t-s} \circ\left\{\left[B \star\left(u_{n}\left(s, \theta\left(t_{1}, \omega\right)\right) \circ u_{n}\left(t_{1}, \omega\right)-u_{n}\left(s+t_{1}, \omega\right)\right)\right]\left(W_{n}^{\prime}(s, \omega)\right)\right\} d s \\
& \quad-\frac{1}{2} \int_{0}^{t} \sum_{k=1}^{\infty} T_{t-s} \circ B_{k}^{2} \circ\left[u_{n}\left(s, \theta\left(t_{1}, \omega\right)\right) \circ u_{n}\left(t_{1}, \omega\right)-u_{n}\left(s+t_{1}, \omega\right)\right] d s,
\end{aligned}
$$

for all $t>0$. The above identity and a simple application of Gronwall's lemma yields

$$
u_{n}\left(t, \theta\left(t_{1}, \omega\right)\right) \circ u_{n}\left(t_{1}, \omega\right)-u_{n}\left(t_{1}+t, \omega\right)=0
$$

for all $t, t_{1} \geq 0$ and all $\omega \in \Omega$. Hence $\left(u_{n}, \theta\right)$ is a perfect cocycle in $L(H)$. Using (1.2.36) and passing to the limit in $L(H)$ as $n \rightarrow \infty$ in the above identity implies that $(u, \theta)$ is a crude $L(H)$-valued cocycle. In order to obtain a perfect version of this cocycle, it is sufficient to prove that there is a sure event $\Omega^{*} \in \mathcal{F}$ (independent of $t_{1} \in \mathbf{R}^{+}$) such that $\theta(t, \cdot)\left(\Omega^{*}\right) \subseteq \Omega^{*}$ for all $t \geq 0$, and there is a subsequence $u_{n^{\prime}}$ of $u_{n}$ such that

$$
\lim _{n^{\prime}, m^{\prime} \rightarrow \infty} \sup _{0<t \leq a}\left\|u_{n^{\prime}}(t, \omega)-u_{m^{\prime}}(t, \omega)\right\|_{L_{2}(H)}^{2}=0
$$

for each $a>0$ and all $\omega \in \Omega^{*}$. Set $v_{n}\left(t_{1}, t, \omega\right):=u_{n}\left(t-t_{1}, \theta\left(t_{1}, \omega\right)\right), t \geq t_{1} \geq 0$. Then $v_{n}$ solves the integral equation

$$
\begin{aligned}
v_{n}\left(t_{1}, t, \omega\right)=T_{t-t_{1}} & +\int_{t_{1}}^{t} T_{t-s} \circ\left\{\left[B \star v_{n}\left(t_{1}, s, \omega\right)\right]\left(W_{n}^{\prime}(s, \omega)\right)\right\} d s \\
& -\frac{1}{2} \int_{t_{1}}^{t} \sum_{k=1}^{\infty} T_{t-s} \circ B_{k}^{2} \circ v_{n}\left(t_{1}, s, \omega\right) d s, \\
v_{n}\left(t_{1}, t_{1}, \omega\right)=I, &
\end{aligned}
$$

for $t \geq t_{1} \geq 0$. The above equation implies that $v_{n}\left(t_{1}, t, \omega\right)$ is continuous in $\left(t_{1}, t\right)$ for each $\omega \in \Omega$. Furthermore, if we apply the approximation scheme (in $L_{2}(H)$ ) to the above integral equation, we get a subsequence $\left\{v_{n^{\prime}}\right\}_{n^{\prime}=1}^{\infty}$ of $\left\{v_{n}\right\}_{n=1}^{\infty}$ such that for a.a. $\omega \in \Omega$

$$
\lim _{n^{\prime}, m^{\prime} \rightarrow \infty} \sup _{0<t_{1} \leq t \leq a}\left\|v_{n^{\prime}}\left(t_{1}, t, \omega\right)-v_{m^{\prime}}\left(t_{1}, t, \omega\right)\right\|_{L_{2}(H)}^{2}=0
$$


for each $a>0$. Now define $\Omega^{*}$ to be the set of all $\omega \in \Omega$ such that the subsequence $\left\{v_{n^{\prime}}\left(t_{1}, t, \omega\right)\right.$ : $\left.n^{\prime} \geq 1\right\}$ converges in $L(H)$ uniformly in $\left(t_{1}, t\right)$ for $0<t_{1} \leq t \leq a$ and all $a>0$. Therefore $\Omega^{*}$ is a $\theta(t, \cdot)$-invariant sure event. Define

$$
u(t, \omega):=\lim _{n^{\prime} \rightarrow \infty} v_{n^{\prime}}(0, t, \omega)
$$

for all $t \geq 0$ and all $\omega \in \Omega^{*}$. Hence $(u, \theta)$ is a perfect cocycle in $L(H)$. This proves assertion (i) of the theorem.

To prove the second assertion of the theorem, fix $s \geq 0$ and define $\hat{v}_{n}(s, t, \omega):=\hat{u}_{n}(t-$ $s, \theta(s, \omega))=u_{n}(t-s, \theta(s, \omega))-T_{t-s}, t \geq s \geq 0$. It is easy to see that $\hat{v}_{n}$ solves the integral equation

$$
\begin{aligned}
\hat{v}_{n}(s, t, \omega) & =\int_{s}^{t} T_{t-\lambda} \circ\left\{\left[B \star \hat{v}_{n}(s, \lambda, \omega)\right]\left(W_{n}^{\prime}(\lambda, \omega)\right)\right\} d \lambda+\int_{s}^{t} T_{t-\lambda} B T_{\lambda-s}\left(W_{n}^{\prime}(\lambda, \omega)\right) d \lambda \\
& -\frac{1}{2} \int_{s}^{t} \sum_{k=1}^{\infty} T_{t-\lambda} \circ B_{k}^{2} \circ \hat{v}_{n}(s, \lambda, \omega) d \lambda, \\
\hat{v}_{n}(s, s, \omega) & =0 \in L_{2}(H),
\end{aligned}
$$

for $t \geq s \geq 0$. The above equation implies that the map $\Delta \ni(s, t) \mapsto \hat{v}_{n}(s, t, \omega) \in L_{2}(H)$ is continuous for each $\omega \in \Omega$. Applying the approximation scheme again, there is a subsequence $\left\{\hat{v}_{n^{\prime}}\right\}_{n^{\prime}=1}^{\infty}$ of $\left\{\hat{v}_{n}\right\}_{n=1}^{\infty}$ such that for a.a. $\omega \in \Omega$, one has

$$
\lim _{n^{\prime}, m^{\prime} \rightarrow \infty} \sup _{0 \leq s \leq t \leq a}\left\|\hat{v}_{n^{\prime}}(s, t, \omega)-\hat{v}_{m^{\prime}}(s, t, \omega)\right\|_{L_{2}(H)}^{2}=0
$$

for each $a>0$. Define $\hat{\Omega}^{*}$ to be the set of all $\omega \in \Omega$ such that the subsequence $\left\{\hat{v}_{n^{\prime}}(s, t, \omega): n^{\prime} \geq 1\right\}$ converges in $L_{2}(H)$ uniformly in $(s, t)$ for $0 \leq s \leq t \leq a$ and all $a>0$. Therefore $\hat{\Omega}^{*}$ is a $\theta(t, \cdot)$-invariant sure event. Define

$$
\hat{u}(t, \omega):=\lim _{n^{\prime} \rightarrow \infty} \hat{v}_{n^{\prime}}(0, t, \omega)
$$

for all $t \geq 0$ and all $\omega \in \hat{\Omega}^{*}$. Therefore, the map $\Delta \ni(s, t) \mapsto \hat{u}(t-s, \theta(s, \omega)) \in L_{2}(H)$ is jointly continuous. In particular, $\sup _{0 \leq s \leq t \leq a}\|\hat{u}(t-s, \cdot, \theta(s, \omega))\|_{L(H)}<\infty$, for all $\omega \in \hat{\Omega}^{*}$ and all $a>0$. Using the fact that $\sup _{0 \leq s \leq t \leq a}\left\|T_{t-s}\right\|_{L(H)}<\infty$, it follows that $u(t, \omega):=\hat{u}(t, \omega)+T_{t}, \omega \in \Omega^{*} \cap \hat{\Omega}^{*}$, gives a version of the cocycle that also satisfies assertion (ii) of the theorem. This completes the proof of the theorem.

Remarks.

(i) Results analogous to Theorem 1.2.4 hold if $B$ is replaced by the an affine linear map $B(x):=$ $B_{0}+B_{1}(x), x \in H$, where $B_{0} \in L(E, H)$ and $B_{1}: H \rightarrow L(E, H)$ satisfies Condition (B). In this case, one gets a cocycle $(u, \theta)$ where each map $u(t, \cdot, \omega): H \rightarrow H$ is of the form $u(t, \cdot, \omega)=u_{0}(t, \cdot, \omega)+u_{1}(t, \omega)$ with $u_{0}(t, \cdot, \omega) \in L_{2}(H)$ and $u_{1}(t, \omega) \in H$ for $t>0, \omega \in \Omega$. This follows using minor modifications of the above arguments. 
(ii) It is possible to replace $B$ in the see (1.2.1) by an adapted random field $B: \mathbf{R}^{+} \times H \times \Omega \rightarrow$ $L(E, H)$ satisfying appropriate integrability and regularity conditions, which is such that $B(t, \cdot, \omega): H \rightarrow L(E, H)$ satisfies Condition (B) for each $t \geq 0, \omega \in \Omega$. The conclusions of Theorems 1.2.1-1.2.3, 1.2.3' will still hold in this case. However, the stochastic semiflow will only satisfy Definition 1.1.1 (rather than the cocycle property in Definition 1.1.2). On the other hand if $B$ is stationary, then the cocycle property should hold (on a suitably enlarged probability space) (Theorem 1.2.4).

(iii) Theorems 1.2.1-1.2.4, 1.2.3' also hold if the operator $A$ is allowed to have a non-zero discrete spectrum $\left\{\mu_{n}: n \geq 1\right\}$ which is bounded below. This yields a splitting $A=A_{0}+A_{1}$ where $\sigma\left(A_{0}\right)$ consists of positive eigenvalues and $\sigma\left(A_{1}\right)$ of finitely many negative eigenvalues.

\section{(b) Semilinear stochastic evolution equations}

In this section, we continue to assume that the operators $A, B$, the cylindrical Brownian motion $W$, the canonical filtered Wiener space $\left(\Omega, \mathcal{F},\left(\mathcal{F}_{t}\right)_{t \geq 0}, P\right)$ and the Brownian shift $\theta: \mathbf{R} \times \Omega \rightarrow$ $\Omega$ are as defined in part (a) of this section and satisfy the conditions therein. The semigroup generated by $-A$ is denoted as before by $T_{t}, t \geq 0$. Furthermore, we let $F: H \rightarrow H$ be a (Fréchet) $C^{1}$ non-linear map satisfying the following locally Lipschitz and linear growth hypotheses:

$$
\left.\begin{array}{c}
|F(v)| \leq C(1+|v|), \quad v \in H \\
\left|F\left(v_{1}\right)-F\left(v_{2}\right)\right| \leq L_{n}\left|v_{1}-v_{2}\right|, \quad v_{i} \in H,\left|v_{i}\right| \leq n, i=1,2,
\end{array}\right\}
$$

for some positive constants $C, L_{n}, n \geq 1$.

Consider the semilinear stochastic evolution equation:

$$
\left.\begin{array}{rl}
d u(t) & =-A u(t) d t+F(u(t)) d t+B u(t) d W(t), \quad t>0, \\
u(0) & =x \in H,
\end{array}\right\}
$$

where the operators $A, B$ satisfy the hypotheses of Theorem 1.2.4.

Our main objective in this section is to establish the existence of a $C^{k}$ perfect cocycle $(U, \theta)$ for the above stochastic evolution equation. First we define a mild solution of (1.2.41) as a family of $\left(\mathcal{B}\left(\mathbf{R}^{+}\right) \otimes \mathcal{F}, \mathcal{B}(H)\right)$-measurable, $\left(\mathcal{F}_{t}\right)_{t \geq 0}$-adapted processes $u(\cdot, x, \cdot): \mathbf{R}^{+} \times \Omega \rightarrow H, x \in H$, satisfying the following stochastic integral equations:

$$
u(t, x, \cdot)=T_{t}(x)+\int_{0}^{t} T_{t-s}(F(u(s, x, \cdot))) d s+\int_{0}^{t} T_{t-s} B u(s, x, \cdot) d W(s), \quad t \geq 0,
$$

a.s. ([D-Z.1], Chapter 7, p. 182).

To fix notation, denote by $\phi: \mathbf{R}^{+} \times \Omega \rightarrow L(H)$ the perfect cocycle generated by the linear stochastic evolution equation

$$
\left.\begin{array}{rl}
d \phi(t) & =-A \phi(t) d t+B \phi(t) d W(t), \quad t>0 \\
\phi(0) & =I \in L(H)
\end{array}\right\}
$$


and obtained via Theorem 1.2.4. That is, $\phi(t, \omega):=u(t, \cdot, \omega) \in L_{2}(H), t>0, \omega \in \Omega$, in the notation of part (a) of this section.

Our first step in the construction of a non-linear cocycle of (1.2.41) is to observe that mild solutions of (1.2.41) correspond to solutions of a random integral equation on $H$. This is shown in the following theorem:

\section{Theorem 1.2.5.}

Suppose the hypotheses of Theorem 1.2.4 are satisfied. Then every $\left(\mathcal{B}\left(\mathbf{R}^{+}\right) \otimes \mathcal{B}(H) \otimes\right.$ $\mathcal{F}, \mathcal{B}(H))$-measurable, $\left(\mathcal{F}_{t}\right)_{t \geq 0}$-adapted solution field $U(t, x, \omega)$ of the $H$-valued random integral equation

$$
U(t, x, \omega)=\phi(t, \omega)(x)+\int_{0}^{t} \phi(t-s, \theta(s, \omega))(F(U(s, x, \omega))) d s, \quad t \geq 0, x \in H,
$$

is a mild solution of the semilinear stochastic evolution equation (1.2.41).

Proof.

Let $U$ be a solution of (1.2.44) with the given measurability properties. It is sufficient to prove that $U(\cdot, x, \cdot)$ satisfies the stochastic integral equation (1.2.42). Substituting from the identity:

$$
\phi(t, \omega)(x)=T_{t}(x)+(\omega) \int_{0}^{t} T_{t-s} B \phi(s, \cdot)(x) d W(s), \quad t \geq 0, x \in H
$$

into (1.2.44), gives the following a.s. relations

$$
\begin{aligned}
U(t, x, \cdot)=T_{t}(x) & +\int_{0}^{t} T_{t-s} B \phi(s, \cdot)(x) d W(s)+\int_{0}^{t} T_{t-s}(F(U(s, x, \cdot))) d s \\
& +\int_{0}^{t} \int_{0}^{t-s} T_{t-s-s^{\prime}} B \phi\left(s^{\prime}, \theta(s, \cdot)\right)(F(U(s, x, \cdot))) d W\left(s^{\prime}, \theta(s, \cdot)\right) d s \\
=T_{t}(x) & +\int_{0}^{t} T_{t-s} B \phi(s, \cdot)(x) d W(s)+\int_{0}^{t} T_{t-s}(F(U(s, x, \cdot))) d s \\
& +\int_{0}^{t} \int_{0}^{t-s} T_{t-s-s^{\prime}} B \phi\left(s^{\prime}, \theta(s, \cdot)\right)(F(U(s, x, \cdot))) d W\left(s^{\prime}+s\right) d s \\
=T_{t}(x) & +\int_{0}^{t} T_{t-s} B \phi(s, \cdot)(x) d W(s)+\int_{0}^{t} T_{t-s}(F(U(s, x, \cdot))) d s \\
& +\int_{0}^{t} \int_{s}^{t} T_{t-\lambda} B \phi(\lambda-s, \theta(s, \cdot))(F(U(s, x, \cdot))) d W(\lambda) d s \\
=T_{t}(x) & +\int_{0}^{t} T_{t-s} B \phi(s, \cdot)(x) d W(s)+\int_{0}^{t} T_{t-s}(F(U(s, x, \cdot))) d s \\
& +\int_{0}^{t} \int_{0}^{\lambda} T_{t-\lambda} B \phi(\lambda-s, \theta(s, \cdot))(F(U(s, x, \cdot))) d s d W(\lambda) \\
=T_{t}(x) & +\int_{0}^{t} T_{t-s}(F(U(s, x, \cdot))) d s
\end{aligned}
$$




$$
\begin{aligned}
& +\int_{0}^{t} T_{t-\lambda} B\left\{\phi(\lambda)(x)+\int_{0}^{\lambda} \phi(\lambda-s, \theta(s, \cdot))(F(U(s, x, \cdot))) d s\right\} d W(\lambda) \\
=T_{t}(x) & +\int_{0}^{t} T_{t-s}(F(U(s, x, \cdot))) d s+\int_{0}^{t} T_{t-\lambda} B U(\lambda, x, \cdot) d W(\lambda)
\end{aligned}
$$

for $t \geq 0$. Hence $U$ satisfies (1.2.42) and is therefore a mild solution of (1.2.41).

Our next theorem shows that the random integral equation (1.2.44) admits a unique $\left(\mathcal{B}\left(\mathbf{R}^{+}\right) \otimes\right.$ $\mathcal{B}(H) \otimes \mathcal{F}, \mathcal{B}(H)$ )-measurable, $\left(\mathcal{F}_{t}\right)_{t \geq 0^{-}}$adapted solution $U: \mathbf{R}^{+} \times H \times \Omega \rightarrow H$. The fact that $(U, \theta)$ is a smooth perfect cocycle can be read off from (1.2.44), as in the proof of Theorem 1.2.6 below.

For any positive integer $j$, denote by $L_{2}^{(j)}(H, H) \subset L^{(j)}(H, H)$ the space of all HilbertSchmidt $j$-multilinear maps $A \in L^{(j)}(H, H)$ given the Hilbert-Schmidt norm

$$
\|A\|_{L_{2}^{(j)}(H, H)}:=\sum_{\substack{n_{i} \geq 1 \\ 1 \leq i \leq j}}\left|A\left(e_{n_{1}}, e_{n_{2}}, \cdots, e_{n_{j}}\right)\right|_{H}^{2}<\infty
$$

where $\left\{e_{n_{i}}: n_{i} \geq 1\right\}$ is a complete orthonormal system in $H$ for each $1 \leq i \leq j$.

\section{Theorem 1.2.6.}

Assume that the operators $A, B$ satisfy the hypotheses of Theorem 1.2.4. Suppose that $F$ satisfies the linear growth and Lipschitz conditions (1.2.40). Then the mild solution of (1.2.41) has a $\left(\mathcal{B}\left(\mathbf{R}^{+}\right) \otimes \mathcal{B}(H) \otimes \mathcal{F}, \mathcal{B}(H)\right)$-measurable version $U: \mathbf{R}^{+} \times H \times \Omega \rightarrow H$ with the following properties:

(i) For each $x \in H, U(\cdot, x, \cdot): \mathbf{R}^{+} \times \Omega \rightarrow H$ is $\left(\mathcal{F}_{t}\right)_{t \geq 0}$-adapted and satisfies (1.2.42) a.s.

(ii) $(U, \theta)$ is a perfect $C^{0,1}$ cocycle (in the sense of Definition 1.2).

(iii) For each $(t, \omega) \in(0, \infty) \times \Omega$, the map $H \ni x \mapsto U(t, x, \omega) \in H$ takes bounded sets into relatively compact sets.

Moreover, if we assume that $F$ is $C^{k, \epsilon}$ on $H$ for a positive integer $k$ and $\epsilon \in(0,1]$, then the mild solution $(U, \theta)$ also enjoys the following properties:

(iv) $(U, \theta)$ is a $C^{k, \epsilon}$ perfect cocycle.

(v) For each $(t, x, \omega) \in \mathbf{R}^{+} \times H \times \Omega$, the Fréchet derivatives $D^{(j)} U(t, x, \omega) \in L_{2}^{(j)}(H, H)$, $1 \leq j \leq k$, and each map

$$
[0, \infty) \times H \times \Omega \ni(t, x, \omega) \mapsto D^{(j)} U(t, x, \omega) \in L^{(j)}(H, H), \quad 1 \leq j \leq k,
$$

is strongly measurable.

(vi) For any positive $a, \rho$,

$$
E \log ^{+}\left\{\sup _{\substack{0 \leq t_{1}, t_{2} \leq a \\ x \in H}} \frac{\left|U\left(t_{2}, x, \theta\left(t_{1}, \cdot\right)\right)\right|}{(1+|x|)}\right\}<\infty
$$


and

$$
E \log ^{+} \sup _{\substack{0 \leq t_{1}, t_{2} \leq a \\|x| \leq \rho, 1 \leq j \leq k}}\left\{\left\|D^{(j)} U\left(t_{2}, x, \theta\left(t_{1}, \cdot\right)\right)\right\|_{L^{(j)}(H, H)}\right\}<\infty
$$

Proof.

In view of Theorem 1.2.5, we construct a version of the mild solution of (1.2.41) by applying the classical technique of successive approximations to the integral equation (1.2.44). Define the sequence $U_{n}: \mathbf{R}^{+} \times H \times \Omega \rightarrow H, n \geq 1$, by

$$
\left.\begin{array}{c}
U_{n+1}(t, x, \omega)=\phi(t, \omega)(x)+\int_{0}^{t} \phi(t-s, \theta(s, \omega))\left(F\left(U_{n}(s, x, \omega)\right)\right) d s, \\
U_{1}(t, x, \omega):=\phi(t, \omega)(x)
\end{array}\right\}
$$

for all $(t, x, \omega) \in \mathbf{R}^{+} \times H \times \Omega$. Fix an arbitrary bounded open set $S$ in $H$. Let $C_{b}^{0}(S, H)$ denote the space of all continuous maps $f: S \rightarrow H$ such that $f(S)$ is relatively compact in $H$. Give $C_{b}^{0}(S, H)$ the supremum norm

$$
\|f\|_{C_{b}^{0}}:=\sup _{x \in S}|f(x)|_{H}, \quad f \in C_{b}^{0}(S, H) .
$$

It is not hard to see that $C_{b}^{0}(S, H)$ is a Banach space. For fixed $\omega \in \Omega$ and any $a>0$, we will view the sequence (1.2.45) as a uniformly convergent sequence of bounded measurable paths $[0, a] \ni t \mapsto$ $U_{n}(t, \cdot, \omega) \in C_{b}^{0}(S, H)$ in the Banach space $C_{b}^{0}(S, H)$. To see this, we use induction on $n$. In view of Theorem 1.2.4 (ii), define the finite random constant $\|\phi\|_{\infty}:=\sup _{0 \leq s \leq t \leq a}\|\phi(t-s, \theta(s, \omega))\|_{L(H)}, \omega \in \Omega$. Let $C$ be the positive constant appearing in (1.2.40). Define

$$
M_{1}=\sup _{x \in S}[|x|+C a]\|\phi\|_{\infty} e^{C\|\phi\|_{\infty} a}, \quad \omega \in \Omega
$$

For some integer $n \geq 1$, consider the following induction hypothesis:

Hypotheses $H(n)$ :

(i) For each $(t, \omega) \in(0, a] \times \Omega, U_{n}(t, \cdot, \omega) \in C_{b}^{0}(S, H)$;

(ii) $\left|U_{n}(t, x, \omega)\right| \leq[|x|+C a]\|\phi\|_{\infty} e^{C\|\phi\|_{\infty} t}$ for all $(t, x, \omega) \in[0, a] \times H \times \Omega$;

(iii) $\left|U_{n+1}(t, x, \omega)-U_{n}(t, x, \omega)\right| \leq C\left[1+\|\phi\|_{\infty}|x|\right] L^{n-1}\|\phi\|_{\infty}^{n} \frac{t^{n}}{n !}, \quad(t, x, \omega) \in[0, a] \times H \times \Omega$, where $L$ is the Lipschitz constant of $F$ on the ball $B\left(0, M_{1}\right) \subset H$.

(iv) $U_{n}: \mathbf{R}^{+} \times H \times \Omega \rightarrow H$ is $\left(\mathcal{B}\left(\mathbf{R}^{+}\right) \otimes \mathcal{B}(H) \otimes \mathcal{F}, \mathcal{B}(H)\right)$-measurable, and for each $x \in H$, $U_{n}(\cdot, x, \cdot): \mathbf{R}^{+} \times \Omega \rightarrow H$ is $\left(\mathcal{F}_{t}\right)_{t \geq 0}$-adapted.

We will first check that $H(1)$ is satisfied. Since $\phi(t, \cdot, \omega): H \rightarrow H$ is continuous linear for each $(t, \omega) \in[0, a] \times \Omega$, it is clear that $H(1)(i)$ and $H(1)(i i)$ are satisfied. Using (1.2.45) and the linear growth property of $F$, it follows that

$$
\left|U_{2}(t, x, \omega)-U_{1}(t, x, \omega)\right| \leq C\|\phi\|_{\infty} \int_{0}^{t}\left[1+|\phi(s, \omega)(x)|_{H}\right] d s \leq C\left[1+\|\phi\|_{\infty}|x|\right]\|\phi\|_{\infty} t,
$$


for all $(t, x, \omega) \in[0, a] \times H \times \Omega$. Therefore, $H(1)($ iii $)$ holds. To see the measurability (inductive hypothesis $H(1)(i v)$ ), use the definition of $U_{1}$ in (1.2.45) and Theorem 1.2.1.

Now assume that $H(n)$ holds for some integer $n \geq 1$. In particular, for each $(t, \omega) \in(0, a] \times \Omega$, $U_{n}(t, \cdot, \omega)$ maps $S$ into a relatively compact set in $H$. Therefore, the map

$$
H \ni x \mapsto \int_{0}^{t} \phi(t-s, \theta(s, \omega))\left(F\left(U_{n}(s, x, \omega)\right) d s \in H\right.
$$

takes $S$ into a relatively compact set in $H$, because, for fixed $(t, \omega) \in(0, a] \times \Omega$, the integrand

$$
H \ni x \mapsto \phi(t-s, \theta(s, \omega))\left(F\left(U_{n}(s, x, \omega)\right)\right) \in H
$$

has the same property, and is uniformly bounded in $(s, x) \in[0, t] \times S(\mathrm{H}(\mathrm{n})(\mathrm{ii}))$. Hence, $U_{n+1}(t, \cdot, \omega)(S)$ is relatively compact in $H$ for each $(t, \omega) \in(0, a] \times \Omega$. Since $U_{n}(t, \cdot, \omega): H \rightarrow H$ is continuous, it is easy to see from (1.2.45) that $U_{n+1}(t, \cdot, \omega): H \rightarrow H$ is also continuous for each $(t, \omega) \in(0, a] \times \Omega$. Hence, $H(n+1)(i)$ is satisfied. Using $H(n)(i i)$, the Lipschitz property of $F$ and (1.2.45), a straightforward computation shows that $H(n+1)(i i i)$ is satisfied. A similar argument, using $H(n)(i i)$, the linear growth property of $F$ and (1.2.45), shows that $H(n+1)(i i)$ also holds. To check $H(n+1)(i v)$, note first that for fixed $s \in[0, t]$, the map $\Omega \ni \omega \mapsto \phi(t-s, \theta(s, \omega)) \in L(H)$ is $\mathcal{F}_{t}$-measurable. This follows from the approximation argument at the end of the proof of Theorem 1.2.4. Hence by $H(n)(i v)$, it follows that for fixed $s \in[0, t]$, the map $\Omega \ni \omega \mapsto \phi(t-s, \theta(s, \omega))\left(F\left(U_{n}(s, x, \omega)\right)\right) \in$ $L(H)$ is $\mathcal{F}_{t^{-}}$-measurable. Hence by $(1.2 .45)$, it is easy to see that $U_{n+1}(t, x, \cdot)$ is $\mathcal{F}_{t^{-}}$-measurable for fixed $(t, x) \in \mathbf{R}^{+} \times H$. Furthermore, the integrand on the right-hand-side of (1.2.45) is jointlymeasurable in $(s, x, \omega)$, and therefore $U_{n+1}(t, \cdot, \cdot)$ is jointly measurable for any fixed $t>0$. By continuity of the path $\mathbf{R}^{+} \ni t \mapsto U_{n+1}(t, x, \omega)$ for fixed $(x, \omega) \in H \times \Omega$, the joint measurability of $U_{n+1}: \mathbf{R}^{+} \times H \times \Omega \rightarrow H$ follows. Hence $H(n+1)(i v)$ is satisfied. Therefore, $H(n)$ holds by induction for all integers $n \geq 1$.

The inequality $H(n)($ iii $)$ implies that the series $\sum_{n=1}^{\infty}\left[U_{n+1}(t, \cdot, \omega)-U_{n}(t, \cdot, \omega)\right]$ converges in $C_{b}^{0}(S, H)$ uniformly in $t \in[0, a]$ for each $\omega \in \Omega$. Therefore, the sequence $\left\{U_{n}(t, \cdot, \omega)\right\}_{n=1}^{\infty}$ converges in $C_{b}^{0}(S, H)$ uniformly in $t \in[0, a]$ for each $\omega \in \Omega$. Its limit

$$
\lim _{n \rightarrow \infty} U_{n}(t, \cdot, \omega)=U_{1}(t, \cdot, \omega)+\sum_{n=1}^{\infty}\left[U_{n+1}(t, \cdot, \omega)-U_{n}(t, \cdot, \omega)\right], \quad(t, \omega) \in[0, a] \times \Omega,
$$

is a solution of the random integral equation (1.2.44). Call this limit $U(t, \cdot, \omega) \in C_{b}^{0}(S, H)$ for $(t, \omega) \in \mathbf{R}^{+} \times \Omega$. It is immediately clear from $H(n)(i v)$ and Theorem 1.2.5 that $U$ satisfies the measurability requirements and assertion (i) of the theorem.

We next show that $U(t, \cdot, \omega): H \rightarrow H$ is $C^{1}$ for fixed $(t, \omega) \in \mathbf{R}^{+} \times \Omega$. For each $(x, y, \omega) \in$ $H \times H \times \Omega$, denote by $z(\cdot, x, y, \omega)$ the unique solution of the random linear integral equation:

$$
\begin{aligned}
z(t, x, y, \omega)=\int_{0}^{t} \phi(t-s, \theta(s, \omega)) D F(U(s, x, \omega)) z(s, x, y, \omega) d s & \\
& +\int_{0}^{t} \phi(t-s, \theta(s, \omega)) D F(U(s, x, \omega)) \phi(s, \omega)(y) d s, \quad t>0 .
\end{aligned}
$$


If we suppress $y \in H$, we can view (1.2.46) as a linear integral equation in $L_{2}(H)$ with a unique solution $[0, \infty) \ni t \mapsto z(t, x, \cdot, \omega) \in L_{2}(H)$ for fixed $(x, \omega) \in H \times \Omega$. This holds easily (by successive approximations) because $D F$ is bounded on bounded subsets of $H$ and $\{U(t, x, \omega) ; 0 \leq$ $t \leq a,|x| \leq M\}$ is bounded for any $M>0$, and $\|\phi\|_{\infty}$ is finite. We claim that $U(t, \cdot, \omega)$ is Fréchet differentiable with Fréchet derivative $D U(t, x, \omega) \in L_{2}(H)$ given by

$$
D U(t, x, \omega)(y)=z(t, x, y, \omega)+\phi(t, \omega)(y), \quad y \in H
$$

for each $(t, x, \omega) \in \mathbf{R}^{+} \times H \times \Omega$. To prove our claim, define

$$
\mu(t, x, y, h, \omega):=U(t, x+h y, \omega)-U(t, x, \omega)-h[z(t, x, y, \omega)+\phi(t, \omega)(y)]
$$

for each $(t, x, y, h, \omega) \in \mathbf{R}^{+} \times H \times H \times \mathbf{R} \times \Omega$. Using (1.2.48), (1.2.44) and (1.2.46), we obtain:

$$
\begin{aligned}
& \mu(t, x, y, h, \omega)=\int_{0}^{t} \phi(t-s, \theta(s, \omega)) D F(U(s, x, \omega)) \mu(s, x, y, h, \omega) d s \\
&+\int_{0}^{t} \phi(t-s, \theta(s, \omega))\left\{\int_{0}^{1} D F[\lambda U(s, x+h y, \omega)+(1-\lambda) U(s, x, \omega)]\right. \\
&\quad-D F(U(s, x, \omega)) d \lambda\}(U(s, x+h y, \omega)-U(s, x, \omega)) d s
\end{aligned}
$$

for all $(t, x, y, h, \omega) \in \mathbf{R}^{+} \times H \times H \times \Omega$. Set

$$
M_{2}=\sup _{s \leq a,|h| \leq 1,|y| \leq 1}\{|U(s, x+h y, \omega)|\}, \quad \omega \in \Omega .
$$

Then $M_{2}$ is finite for each $\omega \in \Omega$, because of $\mathrm{H}(\mathrm{n})(\mathrm{ii})$. Let $L_{1}>0$ be the Lipschitz constant of $D F$ on the ball $B\left(0, M_{2}\right)$, and $\|D F\|$ be the bound of $D F$ on $B\left(0, M_{2}\right)$. Then (1.2.49) implies the following inequality:

$$
\begin{aligned}
|\mu(t, x, y, h, \omega)| \leq & \|\phi\|_{\infty}\|D F\| \int_{0}^{t}|\mu(s, x, y, \omega)| d s \\
& +L_{1}\|\phi\|_{\infty} \int_{0}^{t}|U(s, x+h y, \omega)-U(s, x, \omega)|^{2} d s
\end{aligned}
$$

for all $t \in[0, a], x, y \in H, h \in \mathbf{R},|y|,|h| \leq 1, \omega \in \Omega$. Using (1.2.44) and Gronwall's lemma, it is easy to see that

$$
|U(t, x+h y, \omega)-U(t, x, \omega)| \leq|h|\|\phi\|_{\infty}|y| e^{\|\phi\|_{\infty}\|D F\| t}
$$

for all $t \in[0, a], x, y \in H, h \in \mathbf{R},|y|,|h| \leq 1, \omega \in \Omega$. By (1.2.50), (1.2.51) and another simple application of Gronwall's lemma, we obtain

$$
|\mu(t, x, y, h, \omega)| \leq \frac{|h|^{2}|y|^{2}\|\phi\|_{\infty}^{2} L_{1}}{2\|D F\|}\left[e^{2\|\phi\|_{\infty}\|D F\| a}-1\right] e^{\|\phi\|_{\infty}\|D F\| t}
$$


for all $t \in[0, a], x, y \in H, h \in \mathbf{R},|y|,|h| \leq 1, \omega \in \Omega$. Thus,

$$
\lim _{h \rightarrow 0} \frac{1}{h} \sup _{\substack{|y| \leq 1 \\ 0 \leq t \leq a}}|\mu(t, x, y, h, \omega)|=0
$$

for all $x \in H, \omega \in \Omega$. The above relation shows that $U(t, \cdot, \omega): H \rightarrow H$ is Fréchet differentiable at any $x \in H$ and our claim (1.2.47) holds. Now combining (1.2.46) and (1.2.47), it follows that $D U(t, x, \omega)$ satisfies the $L(H)$-valued integral equation:

$$
D U(t, x, \omega)=\phi(t, \omega)+\int_{0}^{t} \phi(t-s, \theta(s, \omega)) D F(U(s, x, \omega)) D U(s, x, \omega) d s
$$

for each $(t, x, \omega) \in \mathbf{R}^{+} \times H \times \Omega$. In the above integral equation, the "coefficients"

$$
\begin{gathered}
{[0, \infty) \times \Omega \ni(t, \omega) \mapsto \phi(t, \omega) \in L(H)} \\
\Delta \times H \times \Omega \ni(s, t, x, \omega) \mapsto \phi(t-s, \theta(s, \omega)) D F(U(s, x, \omega)) \in L(H)
\end{gathered}
$$

are jointly measurable, where $\Delta=\left\{(s, t) \in \mathbf{R}^{2}: 0 \leq s \leq t\right\}$. Therefore, the solution map

$$
[0, \infty) \times H \times \Omega \ni(t, x, \omega) \mapsto D U(t, x, \omega) \in L(H)
$$

is jointly measurable. Furthermore, by continuity of the map $H \ni x \mapsto D F(U(s, x, \omega)) \in L(H, \mathbf{R})$ it follows from (1.2.54) that the map $H \ni x \mapsto D U(t, x, \omega) \in L(H)$ is continuous for fixed $t>0$ and $\omega \in \Omega$. Thus $U(t, \cdot, \omega): H \rightarrow H$ is $C^{1}$. (In fact, the map $H \ni x \mapsto D U(t, x, \omega) \in L_{2}(H), t>0$, is continuous because of the continuity of the map $H \ni x \mapsto z(t, x, \cdot, \omega) \in L_{2}(H)$ in the $L_{2}(H)$-valued integral equation underlying (1.2.46).)

Suppose further that $F$ is $C^{k, \epsilon}, k \geq 1, \epsilon \in(0,1]$. For $k=1$, assertion (vi) of the theorem follows from (1.2.44), the linear growth property of $F$, (1.2.54), Gronwall's lemma and the fact that $E\|\phi\|_{\infty}<\infty$. By suppressing $y$ in (1.2.46) and taking higher-order Fréchet derivatives with respect to $x$ of the underlying $L_{2}(H)$-valued integral equation, assertions (v) and (vi) can be established by induction on $k>1$.

It remains to prove that $(U, \theta)$ is a perfect cocycle on $H$. We use uniqueness of solutions of (1.2.44). Fix $t_{1} \geq 0, \omega \in \Omega$ and $x \in H$. It is sufficient to prove that

$$
U\left(t+t_{1}, x, \omega\right)=U\left(t, U\left(t_{1}, x, \omega\right), \theta\left(t_{1}, \omega\right)\right)
$$

for all $t \geq 0$. Define the two mappings $y, z:[0, \infty) \rightarrow H$ by

$$
y(t):=U\left(t, U\left(t_{1}, x, \omega\right), \theta\left(t_{1}, \omega\right)\right), \quad z(t):=U\left(t+t_{1}, x, \omega\right)
$$


for all $t \geq 0$. Since $U$ satisfies (1.2.44), it follows that

$$
\begin{gathered}
y(t)=\phi\left(t, \theta\left(t_{1}, \omega\right)\right)\left(U\left(t_{1}, x, \omega\right)\right)+\int_{0}^{t} \phi\left(t-s, \theta\left(s, \theta\left(t_{1}, \omega\right)\right)\right)\left(F\left(U\left(s, U\left(t_{1}, x, \omega\right), \theta\left(t_{1}, \omega\right)\right)\right) d s\right. \\
=\phi\left(t, \theta\left(t_{1}, \omega\right)\right)\left(\phi\left(t_{1}, \omega\right)(x)\right)+\int_{0}^{t_{1}} \phi\left(t, \theta\left(t_{1}, \omega\right)\right)\left\{\phi\left(t_{1}-s, \theta(s, \omega)\right)(F(U(s, x, \omega)))\right\} d s \\
\quad+\int_{t_{1}}^{t+t_{1}} \phi\left(t+t_{1}-s, \theta(s, \omega)\right)\left(F\left(y\left(s-t_{1}\right)\right)\right) d s \\
=\phi\left(t+t_{1}, \omega\right)(x)+\int_{0}^{t_{1}} \phi\left(t+t_{1}-s, \theta(s, \omega)\right)(F(U(s, x, \omega))) d s \\
\quad+\int_{t_{1}}^{t+t_{1}} \phi\left(t+t_{1}-s, \theta(s, \omega)\right)\left(F\left(y\left(s-t_{1}\right)\right)\right) d s
\end{gathered}
$$

for all $t \geq 0$. Making the substitution $t^{\prime}:=t+t_{1}$, the above relation yields

$$
\begin{aligned}
y\left(t^{\prime}-t_{1}\right)=\phi\left(t^{\prime}, \omega\right)(x)+\int_{0}^{t_{1}} \phi\left(t^{\prime}-s, \theta(s, \omega)\right)(F(U(s, x, \omega))) d s \\
+\int_{t_{1}}^{t^{\prime}} \phi\left(t^{\prime}-s, \theta(s, \omega)\right)\left(F\left(y\left(s-t_{1}\right)\right)\right) d s, \quad t^{\prime}>t_{1} .
\end{aligned}
$$

Using (1.2.44) and the definition of $z$, it follows that

$$
\begin{aligned}
z(t)=\phi(t+ & \left.t_{1}, \omega\right)(x)+\int_{0}^{t_{1}} \phi\left(t+t_{1}-s, \theta(s, \omega)\right)(F(U(s, x, \omega))) d s \\
& +\int_{t_{1}}^{t+t_{1}} \phi\left(t+t_{1}-s, \theta(s, \omega)\right)(F(U(s, x, \omega))) d s \quad t \geq 0 .
\end{aligned}
$$

Therefore,

$$
\begin{aligned}
z\left(t^{\prime}-t_{1}\right)=\phi\left(t^{\prime}, \omega\right)(x)+\int_{0}^{t_{1}} \phi\left(t^{\prime}-s, \theta(s, \omega)\right)(F(U(s, x, \omega))) d s \\
+\int_{t_{1}}^{t^{\prime}} \phi\left(t^{\prime}-s, \theta(s, \omega)\right)\left(F\left(z\left(s-t_{1}\right)\right)\right) d s, \quad t^{\prime} \geq t_{1} .
\end{aligned}
$$

It is easy to see that (1.2.57) and (1.2.58) imply

$$
\begin{aligned}
\left|y\left(t^{\prime}-t_{1}\right)-z\left(t^{\prime}-t_{1}\right)\right| & \leq \int_{t_{1}}^{t^{\prime}}\left\|\phi\left(t^{\prime}-s, \theta(s, \omega)\right)\right\| \cdot\left|F\left(y\left(s-t_{1}\right)\right)-F\left(z\left(s-t_{1}\right)\right)\right| d s \\
& \left.\leq L\|\phi\|_{\infty} \int_{t_{1}}^{t^{\prime}} \mid y\left(s-t_{1}\right)\right)-z\left(s-t_{1}\right) \mid d s, \quad t_{1} \leq t^{\prime} \leq t_{1}+a,
\end{aligned}
$$

where $L$ is the Lipschitz constant of $F$ on the bounded set $\{y(s), z(s), 0 \leq s \leq a\}$. From the above inequality, we get $y\left(t^{\prime}-t_{1}\right)-z\left(t^{\prime}-t_{1}\right)=0$ for all $t^{\prime} \geq t_{1}$. Hence, $y(t)=z(t)$ for all $t \geq 0$. This implies the perfect cocycle property (1.2.55) and completes the proof of the theorem. 
Remarks.

(i) From the proof of Theorem 1.2.6, it is easy to see that the assertions of the theorem still hold if one replaces the linear growth condition $F$ by the condition that $F$ carries bounded sets in $H$ into bounded sets, and $U(\cdot, \cdot, \omega)$ is bounded on bounded subsets of $[0, \infty) \times H$.

(ii) In (1.2.41), it is possible to replace $F$ by a time-dependent $\tilde{F}: \mathbf{R}^{+} \times H \rightarrow H$ of class $C^{k, \epsilon}$ in the second variable uniformly with respect to $t$ in compacta. This gives a $C^{k, \epsilon}$ semiflow $V: \Delta \times H \times \Omega \rightarrow H$ in the sense of Definition 1.1.1.

Example. Let $\mathcal{D}$ denote the bounded domain in $\mathbf{R}^{d}$ defined by

$$
\mathcal{D}:=\left\{\left(x_{1}, x_{2}, \cdots, x_{d}\right) ; 0 \leq x_{i} \leq \pi, 1 \leq i \leq d\right\}
$$

Equip the operator $B=-\Delta+I$ on $D$ with Neumann boundary and consider the following SPDE:

$$
d u(t, x)=-A u(t, x) d t+f(u(t, x)) d t+c(x) u(t, x) d W(t)
$$

Where $W$ stands for a standard (one-dimensional) Brownian motion, and $A:=B^{\alpha}$ for some positive constant $\alpha$. Let $\phi_{0}(x), \phi_{n}(x)$ be functions on $[0, \pi]$ defined by

$$
\phi_{0}(x)=\left(\frac{1}{\pi}\right)^{\frac{1}{2}}, \quad \phi_{n}(x)=\left(\frac{2}{\pi}\right)^{\frac{1}{2}} \cos (n x), \quad n \geq 1 .
$$

For any non-negative integers $i_{1}, i_{2}, \ldots, i_{d}$, define

$$
\psi_{i_{1}, i_{2}, \ldots, i_{d}}\left(x_{1}, x_{2}, . ., x_{d}\right):=\phi_{i_{1}}\left(x_{1}\right) \phi_{i_{2}}\left(x_{2}\right) \cdots \phi_{i_{d}}\left(x_{d}\right), \quad x_{i} \in[0, \pi], 1 \leq i \leq d .
$$

Then the family $\left\{\psi_{i_{1}, i_{2}, \ldots, i_{d}}: 0 \leq i_{1}, i_{2}, \ldots, i_{d}<\infty\right\}$ forms an orthonormal basis of $L^{2}(\mathcal{D})$. It is easy to verify that each $\psi_{i_{1}, i_{2}, \ldots, i_{d}}$ is an eigenfunction of $B$ with corresponding eigenvalue

$$
\lambda_{i_{1}, i_{2}, \ldots, i_{d}}=1+\sum_{j=1}^{d} i_{j}^{2} .
$$

Thus

$$
A \psi_{i_{1}, i_{2}, \ldots, i_{d}}=\lambda_{i_{1}, i_{2}, \ldots, i_{d}}^{\alpha} \psi_{i_{1}, i_{2}, \ldots, i_{d}}
$$

If $\alpha>\frac{d}{2}$, we have

$$
\begin{aligned}
\sum_{i_{1}, i_{2}, \cdots, i_{d}=0}^{\infty} \frac{1}{\lambda_{i_{1}, i_{2}, \ldots, i_{d}}^{\alpha}} & =\sum_{i_{1}, i_{2}, \cdots, i_{d}=0}^{\infty}\left(1+\sum_{j=1}^{d} i_{j}^{2}\right)^{-\alpha} \\
& \leq \sum_{i_{1}, i_{2}, \cdots, i_{d}=0}^{\infty} \prod_{j=1}^{d}\left(\frac{1}{d}+i_{j}^{2}\right)^{-\frac{\alpha}{d}} \\
& =\left\{\sum_{k=0}^{\infty}\left(\frac{1}{d}+k^{2}\right)^{-\frac{\alpha}{d}}\right\}^{d}<\infty .
\end{aligned}
$$

If $f$ is Lipschitz and $c(x)$ is bounded, then the assumptions in Theorems 1.2.1 and 1.2.6 are satisfied. So Theorem 1.2.6 applies.

In the next section, we will see more applications of the results established in this section. 


\subsection{Semilinear stochastic partial differential equations: Lipschitz nonlinearity.}

Let $\mathcal{D}$ be a smooth bounded domain in $\mathbf{R}^{d}$. Consider the Laplacian operator:

$$
\Delta:=\sum_{i=1}^{d} \frac{\partial^{2}}{\partial \xi_{i}^{2}}
$$

defined on $\mathcal{D}$. Let $H:=H_{0}^{k}(\mathcal{D})$ be the Sobolev space of order $k>d / 2$, i.e., the completion of $C_{0}^{\infty}(\mathcal{D})$ under the Sobolev norm

$$
\|u\|_{H_{0}^{k}}^{2}:=\sum_{|\alpha| \leq k} \int_{\mathcal{D}}\left|D^{\alpha} u(\xi)\right|^{2} d \xi,
$$

where $d \xi$ denotes $d$-dimensional Lebesgue measure on $\mathbf{R}^{d}$.

Consider the spde

$$
\left.\begin{array}{rl}
d u(t) & =\frac{1}{2} \Delta u(t) d t+f(u(t)) d t+\sum_{i=1}^{\infty} \sigma_{i} u(t) d W_{i}(t), \quad t>0 \\
u(0) & =\psi \in H_{0}^{k}(\mathcal{D}) \\
\left.u(t)\right|_{\partial \mathcal{D}} & =0, \quad t \geq 0,
\end{array}\right\}
$$

where $f: \mathbf{R} \rightarrow \mathbf{R}$ is a $C_{b}^{\infty}$ function, the $\sigma_{i}: \mathcal{D} \rightarrow \mathbf{R}, i \geq 1$, are functions in the Sobolev space $H_{0}^{s}(\mathcal{D})$ with $s>k+\frac{d}{2}$, and the $W_{i}, i \geq 1$, are standard independent one-dimensional Brownian motions. Assume that the coefficients $\sigma_{i}$ in (1.3.2) satisfy the following condition

$$
\sum_{i=1}^{\infty}\left\|\sigma_{i}\right\|_{H_{0}^{s}}^{2}<\infty
$$

Denote by $C_{0}^{\infty}(\mathcal{D})$ the set of all smooth test functions $\phi: \mathcal{D} \rightarrow \mathbf{R}$ which vanish on $\partial \mathcal{D}$. Let $L^{\infty}(\mathcal{D})$ stand for all essentially bounded measurable functions $\psi: \mathcal{D} \rightarrow \mathbf{R}$ with the usual norm

$$
\|\psi\|_{\infty}:=\operatorname{essup}_{\xi \in \mathcal{D}}|\psi(\xi)|
$$

An $\left(\mathcal{F}_{t}\right)_{t \geq 0^{-}}$adapted random field $u: \mathbf{R}^{+} \times \mathcal{D} \times \Omega \rightarrow \mathbf{R}$ is a weak solution of (1.3.2) if $u(t, \cdot, \omega) \in$ $H_{0}^{k}(\mathcal{D})$ for a.a. $\omega \in \Omega$ and the following identity holds:

$$
\left.\begin{array}{rl}
d<u(t), \phi>_{L^{2}} & =\nu<u(t), \Delta \phi>_{L^{2}} d t+<f(u(t)), \phi>_{L^{2}} d t+\sum_{i=1}^{\infty}<\sigma_{i} u(t), \phi>_{L^{2}} d W_{i}(t), \\
u(0) & =\psi \in H_{0}^{k}(\mathcal{D}), \\
\left.u(t)\right|_{\partial \mathcal{D}}=0, \quad t>0 &
\end{array}\right\}
$$

for all $\phi \in C_{0}^{\infty}(\mathcal{D})$ a.s. In the above equality, $\langle\cdot, \cdot\rangle_{L^{2}}$ denotes the inner product on the Hilbert space $L^{2}(\mathcal{D})$ of all square-integrable functions $\psi: \mathcal{D} \rightarrow \mathbf{R}$, viz.

$$
<\psi_{1}, \psi_{2}>_{L^{2}}:=\int_{\mathcal{D}} \psi_{1}(\xi) \psi_{2}(\xi) d \xi, \quad \psi_{1}, \psi_{2} \in L^{2}(\mathcal{D})
$$


Recall that $d \xi$ stands for $d$-dimensional Lebesgue measure.

We will show that (1.3.2) admits a unique weak solution $u(t) \in H$ a.s., $t>0$, for each $\psi \in H$. Furthermore, the ensemble of all weak solutions of (1.3.2) generates a a $C^{\infty}$ perfect cocycle (also denoted by the same symbol) $u: \mathbf{R}^{+} \times H \times \Omega \rightarrow H$ satisfying the assertions of Theorem 1.3.5 below. In particular, the stochastic semiflow $u(t, \cdot, \omega): H \rightarrow H$ takes bounded sets into relatively compact sets in $H$.

In this section and for the rest of the article, we should emphasize that although the weak solution $u: \mathbf{R}^{+} \times \mathcal{D} \times \Omega \rightarrow \mathbf{R}$ of (1.3.2) and the associated stochastic semiflow $u: \mathbf{R}^{+} \times H \times \Omega \rightarrow H$ are denoted by the same symbol $u$, the distinction between the two notions should be clear from the context.

Set $A:=-\frac{1}{2} \Delta$ with Dirichlet boundary conditions on $\partial \mathcal{D}$. We will view the spde (1.3.2) as a semilinear stochastic evolution equation in $H$ of the form (1.2.41) (Section 1.2). First, define the Nemytskii operator

$$
F(u)(\xi):=f(u(\xi)), \quad \xi \in \mathcal{D}, u \in H .
$$

In Lemma 1.3.3 below, we will show that $F$ is a $C^{\infty}$ map $H \rightarrow H$. Secondly, apply the GrammSchmidt orthogonalization process to the sequence $\left\{\sigma_{i}\right\}_{i=1}^{\infty}$ in $H_{0}^{s}(\mathcal{D})$. This gives an orthonormal family $\left\{f_{i}\right\}_{i=1}^{\infty}$ in $H_{0}^{s}(\mathcal{D})$. Denote by $K$ the closed linear span of $\left\{f_{i}\right\}_{i=1}^{\infty}$ in $H_{0}^{s}(\mathcal{D})$. The reader may check that $K$ is a closed subspace of the Hilbert space $H_{0}^{S}(\mathcal{D})$, and there is a separable Hilbert space $E$ such that $K \subset E$ is a Hilbert-Schmidt embedding (e.g. take $E=L^{2}(\mathcal{D})$ ).

Define the process

$$
W(t):=\sum_{i=1}^{\infty} W_{i}(t) f_{i}, \quad t>0 .
$$

Then it follows from (1.3.3) that $W$ is an $E$-valued cylindrical Brownian motion on the canonical filtered Wiener space $\left(\Omega, \mathcal{F},\left(\mathcal{F}_{t}\right)_{t \in \mathbf{R}}, P\right)$, and with covariance space $K$ (cf. section 1.2). Denote by $\theta: \mathbf{R} \times \Omega \rightarrow \Omega$ the standard $P$-preserving (ergodic) Brownian shift. It is easy to see that $(W, \theta)$ is a perfect helix on $E$ :

$$
W\left(t_{1}+t_{2}, \omega\right)=W\left(t_{2}, \theta\left(t_{1}, \omega\right)\right)-W\left(t_{1}, \omega\right), \quad t_{1}, t_{2} \in \mathbf{R}, \omega \in \Omega .
$$

Define the continuous linear operator $B: H \rightarrow L_{2}(K, H)$ by setting

$$
B(u)\left(f_{i}\right):=\sigma_{i} u, \quad u \in H=H_{0}^{k}(\mathcal{D}), i \geq 1 .
$$

In view of the continuous linear (Sobolev) embedding

$$
H_{0}^{s}(\mathcal{D}) \hookrightarrow C^{k}(\mathcal{D})
$$

it is easy to see that $B \in L\left(H, L_{2}(K, H)\right)$ and satisfies Condition (B) of section 1.2(a). Thirdly, observe that weak solutions of the spde (1.3.2) correspond to mild solutions of the semilinear see:

$$
\left.\begin{array}{rl}
d u(t) & =-A u(t) d t+F(u(t)) d t+B u(t) d W(t), \quad t>0 \\
u(0) & =\psi \in H:=H_{0}^{k}(\mathcal{D})
\end{array}\right\}
$$


([D-Z.1], p. 156).

Finally, we will establish a perfect $C^{\infty}$-cocycle on the Sobolev space $H=H_{0}^{k}(\mathcal{D})$ for mild solutions of the semilinear see (1.3.2'), and hence for weak solutions of the spde (1.3.2).

We begin with some preparation. Following standard notation, let $\alpha$ be a d-tuple of nonnegative integers, viz. $\alpha:=\left(\alpha_{1}, \alpha_{2}, \cdots, \alpha_{d}\right)$ and denote $|\alpha|:=\alpha_{1}+\alpha_{2}+\cdots+\alpha_{d}$. For any $\phi \in C^{|\alpha|}(\mathcal{D})$, denote

$$
\left(D^{(\alpha)} \phi\right)(\xi) \equiv \phi^{(\alpha)}(\xi):=\partial_{1}^{\alpha_{1}} \partial_{2}^{\alpha_{2}} \cdots \partial_{d}^{\alpha_{d}} \phi(\xi), \quad \xi \in \mathcal{D}
$$

and for any integer $l>0$, define

$$
\left\|D^{l} \phi\right\|_{L^{2}}:=\sum_{|\alpha|=l}\left\|D^{(\alpha)} \phi\right\|_{L^{2}}
$$

\section{Lemma 1.3.1.}

Let $\beta_{1}, \cdots, \beta_{\mu}$ be d-tuples and $|\alpha|=\left|\beta_{1}\right|+\left|\beta_{2}\right|+\cdots+\left|\beta_{\mu}\right|$, then there exists a constant $c>0$ such that

$$
\begin{aligned}
& \left\|f_{1}^{\left(\beta_{1}\right)} f_{2}^{\left(\beta_{2}\right)} \cdots f_{\mu}^{\left(\beta_{\mu}\right)}\right\|_{L^{2}} \\
& \quad \leq\left. c^{\mu}|| f_{1}\left\|_{L^{\infty}}^{1-\frac{\left|\beta_{1}\right|}{|\alpha|}}\right\| f_{2}\left\|_{L^{\infty}}^{1-\frac{\left|\beta_{2}\right|}{|\alpha|}} \cdots\right\| f_{\mu}\left\|_{L^{\infty}}^{1-\frac{\mid \beta \mu}{|\alpha|}}\right\| D^{|\alpha|} f_{1}\right|_{L^{2}} ^{\frac{\left|\beta_{1}\right|}{|\alpha|}}\left\|D^{|\alpha|} f_{2}\right\|_{L^{2}}^{\frac{\left|\beta_{2}\right|}{|\alpha|}}\left\|D^{|\alpha|} f_{\mu}\right\|_{L^{2}}^{\frac{|\beta \mu|}{|\alpha|}}
\end{aligned}
$$

A proof of this lemma is given in [Ta], using Gagliardo-Nirenberg-Moser estimates.

\section{Lemma 1.3.2.}

Let $F$ be smooth and assume $F(0)=0$. Then there is a positive constant $c$ such that

$$
\|F(u)\|_{H_{0}^{k}(\mathcal{D})} \leq c C_{k}\left(\|u\|_{L^{\infty}}\right)\left(1+\|u\|_{L^{\infty}}\right)^{k-1}\|u\|_{H_{0}^{k}(\mathcal{D})},
$$

for $u \in H_{0}^{k}(\mathcal{D}) \cap L^{\infty}$, where

$$
C_{k}(\lambda)=\sup _{|u| \leq \lambda, \quad 1 \leq \mu \leq k}\left|F^{(\mu)}(u)\right| .
$$

Proof.

We need only prove the assertion of the lemma for $u \in C_{0}^{\infty}(\mathcal{D})$. The chain rule gives for any $d$-tuple $\alpha$ with $1 \leq|\alpha| \leq k$,

$$
D^{\alpha} F(u)=\sum_{\beta_{1}+\beta_{2}+\cdots+\beta_{\mu}=\alpha, \mu \geq 1} c_{\beta} u^{\left(\beta_{1}\right)} u^{\left(\beta_{2}\right)} \cdots u^{\left(\beta_{\mu}\right)} F^{(\mu)}(u) .
$$

Hence

$$
\left\|D^{\alpha} F(u)\right\|_{L^{2}} \leq C_{k}\left(\|u\|_{L^{\infty}}\right) \sum_{\beta_{1}+\beta_{2}+\cdots+\beta_{\mu}=\alpha, \mu \geq 1} c_{\beta}\left\|u^{\left(\beta_{1}\right)} u^{\left(\beta_{2}\right)} \cdots u^{\left(\beta_{\mu}\right.}\right\|_{L^{2}} .
$$


Applying Lemma 1.3.1 to $f_{i}=u, i=1,2, \cdots, \mu$, we have

$$
\left\|u^{\left(\beta_{1}\right)} u^{\left(\beta_{2}\right)} \cdots u^{\left(\beta_{\mu}\right)}\right\|_{L^{2}} \leq c^{\mu}\|u\|_{L^{\infty}}^{\mu-1}\left\|D^{|\alpha|} u\right\|_{L^{2}} .
$$

Therefore,

$$
\begin{aligned}
\sum_{\beta_{1}+\beta_{2}+\cdots+\beta_{\mu}=\alpha, \mu \geq 1} c_{\beta}\left\|u^{\left(\beta_{1}\right)} u^{\left(\beta_{2}\right)} \cdots u^{\left(\beta_{\mu}\right)}\right\|_{L^{2}} & \leq \sum_{\beta_{1}+\beta_{2}+\cdots+\beta_{\mu}=\alpha, \mu \geq 1} c_{\beta} c^{\mu}\|u\|_{L^{\infty}}^{\mu-1}\left\|D^{|\alpha|} u\right\|_{L^{2}} \\
& \leq c|| D^{|\alpha|} u\left\|_{L^{2}} \sum_{1 \leq \mu \leq|\alpha|} C_{\mu}^{|\alpha|}\right\| u \|_{L^{\infty}}^{\mu-1} \\
& =c|| D^{|\alpha|} u \|_{L^{2}}\left(1+\|u\|_{L^{\infty}}\right)^{|\alpha|-1} \\
& \leq c\|u\|_{H^{k}}\left(1+\|u\|_{L^{\infty}}\right)^{k-1}
\end{aligned}
$$

for a constant $c>0$. Note also that

$$
\|F(u)\|_{L^{2}} \leq C_{k}\|u\|_{L^{2}} \leq C_{k}\|u\|_{H^{k}}, \quad u \in C_{0}^{\infty}(\mathcal{D})
$$

The assertion of the lemma follows easily from the above inequality.

\section{Lemma 1.3.3.}

Suppose $k>\frac{d}{2}$, and $f: \mathbf{R} \rightarrow \mathbf{R}$ is a $C^{\infty}$ function. Then the function $F: H_{0}^{k}(\mathcal{D}) \rightarrow H_{0}^{k}(\mathcal{D})$ defined by $\left(1.3 .3^{\prime}\right)$ is a $C^{\infty}$ map from $H_{0}^{k}(\mathcal{D})$ into $H_{0}^{k}(\mathcal{D})$.

Proof.

Recall the following Sobolev embeddings

$$
\begin{aligned}
H_{0}^{r}(\mathcal{D}) \hookrightarrow L^{\frac{2 d}{d-2 r}}(\mathcal{D}), & r<\frac{d}{2}, \\
H_{0}^{r}(\mathcal{D}) \hookrightarrow L^{\infty}(\mathcal{D}), & r>\frac{d}{2} .
\end{aligned}
$$

Let us first prove that $F \in C^{1}(H, H)$, where $H:=H_{0}^{k}(\mathcal{D})$. Fix $u \in H$. We will show that $F$ is Fréchet differentiable and $D F(u)(h)(\xi) \equiv S_{u}(h)(\xi)=f^{\prime}(u(\xi)) h(\xi), h \in H, \xi \in \mathcal{D}$. To prove this, note that only functions in some ball $B(0, \delta) \subset H$ centered at 0 are involved. By the Sobolev embedding theorem, the range of functions in $B(0, \delta)$ is contained in a compact interval in $\mathbf{R}$. Thus, we can assume $f \in C_{b}^{\infty}$ in the sequel. We start by proving that $S_{u}(h) \in H$ for $h \in H$. Let $r \leq k$. By the chain and product rules, it follows that $\left(S_{u}(h)\right)^{(r)}$ can be written as a finite sum whose general term is of the form: $C(\xi) u^{\left(l_{1}\right)}(\xi) \cdots u^{\left(l_{m}\right)}(\xi) h^{\left(j_{1}\right)}(\xi) \cdots h^{\left(j_{n}\right)}(\xi)$, where $C(\cdot) \in L^{\infty}(\mathcal{D})$, and $l_{1}+\cdots+l_{m}+j_{1}+\cdots j_{n}=r$. Since $u^{(l)} \in H_{0}^{k-l}(\mathcal{D})$ and $h^{(j)} \in H_{0}^{k-j}(\mathcal{D})$, the Sobolev embedding theorem implies that $u^{(l)} \in L^{\frac{2 d}{d-2 k+2 l}}(\mathcal{D})$ and $h^{(j)} \in L^{\frac{2 d}{d-2 k+2 j}}(\mathcal{D})$. As

$$
\sum_{i=1}^{m}\left(d-2 k+2 l_{i}\right)+\sum_{i=1}^{n}\left(d-2 k+2 j_{i}\right)-d \leq(m+n-1)(d-2 k)<0,
$$


we have

$$
\frac{\sum_{i=1}^{m}\left(d-2 k+2 l_{i}\right)}{2 d}+\frac{\sum_{i=1}^{n}\left(d-2 k+2 j_{i}\right)}{2 d} \leq \frac{1}{2} .
$$

By Hölder's inequality and the Sobolev embedding theorem, this implies that

$$
\left|C(\cdot) u^{\left(l_{1}\right)}(\cdot) \cdots u^{\left(l_{m}\right)}(\cdot) h^{\left(j_{1}\right)}(\cdot) \cdots h^{\left(j_{n}\right)}(\cdot)\right|_{L^{2}(\mathcal{D})} \leq c|u|_{H}^{m}|h|_{H}^{n} .
$$

where $c$ is a positive constant. Thus $S_{u}(h)$ is not only in $H$, but the map $H \ni h \mapsto S_{u}(h) \in H$ is a continuous linear operator. Now

$$
F(u+t h)(\xi)-F(u)(\xi)-t S_{u}(h)(\xi)=\int_{0}^{t}\left[f^{\prime}(u(\xi)+s h(\xi))-f^{\prime}(u(\xi))\right] h(\xi) d s
$$

for each $\xi \in \mathcal{D}, u, h \in H, t \geq 0$. To show that $D F(u)=S_{u}$, we need to prove that

$$
\lim _{t \rightarrow 0} \sup _{|h|_{H} \leq 1}\left|\frac{1}{t} \int_{0}^{t}\left[f^{\prime} \circ(u+s h)-f^{\prime} \circ(u)\right] \cdot h d s\right|_{H}=0 .
$$

It is sufficient to establish

$$
\lim _{s \rightarrow 0} \sup _{|h|_{H} \leq 1}\left|\left[f^{\prime} \circ(u+s h)-f^{\prime} \circ(u)\right] \cdot h\right|_{H}=0 .
$$

The above relation will hold if we show that

$$
\lim _{s \rightarrow 0} \sup _{|h|_{H} \leq 1}\left|\left[\left(f^{\prime} \circ(u+s h)-f^{\prime} \circ(u)\right) \cdot h\right]^{(r)}\right|_{L^{2}(\mathcal{D})}=0
$$

for $r \leq k$.

Elementary computations show that $\left[\left(f^{\prime} \circ(u+s h)-f^{\prime} \circ(u)\right) \cdot h\right]^{(r)}(\xi)$ is a finite sum consisting of terms which are either of the form

$$
G_{1}(\xi, h, s):=\left(f^{(l)} \circ(u+s h)-f^{(l)} \circ(u)\right)(\xi) u^{\left(l_{1}\right)}(\xi) \ldots u^{\left(l_{m}\right)}(\xi) h^{\left(j_{1}\right)}(\xi) \ldots h^{\left(j_{n}\right)}(\xi), l \leq r+1
$$

or of the form

$$
G_{2}(\xi, h, s):=s^{a} C(\xi) u^{\left(l_{1}\right)}(\xi) \ldots u^{\left(l_{m}\right)}(\xi) h^{\left(j_{1}\right)}(\xi) \ldots h^{\left(j_{n}\right)}(\xi), a \geq 1, C(\cdot) \in L^{\infty}(\mathcal{D}),
$$

where $l_{1}+\ldots+l_{m}+j_{1}+\ldots+j_{n}=r, h \in H, s>0$. For terms like $G_{1}$, using the Lipschitz continuity of $f^{(l)}$, it follows that

$$
\left|G_{1}(\xi, h, s)\right| \leq c s|h|_{L^{\infty}(\mathcal{D})}\left|u^{\left(l_{1}\right)}(\xi) \ldots u^{\left(l_{m}\right)}(\xi) h^{\left(j_{1}\right)}(\xi) \ldots h^{\left(j_{n}\right)}(\xi)\right|, \quad \xi \in \mathcal{D}, h \in H, s>0 .
$$

Using Hölder's inequality and the Sobolev embedding theorem, and arguing as in the proof of $S_{u}(h) \in H$, we obtain the following estimate

$$
\| G_{1}\left(\cdot, h, s \|_{L^{2}(\mathcal{D})} \leq c s|u|_{H}^{m}|h|_{H}^{n+1}\right.
$$


where $c$ is a positive constant and $\xi \in \mathcal{D}, h \in H, s>0$. Hence,

$$
\lim _{s \rightarrow 0} \sup _{|h|_{H} \leq 1}\left\|G_{1}(\cdot, h, s)\right\|_{L^{2}(\mathcal{D})}=0
$$

Similar arguments lead also to

$$
\lim _{s \rightarrow 0} \sup _{|h|_{H} \leq 1}\left\|G_{2}(\cdot, h, s)\right\|_{L^{2}(\mathcal{D})}=0
$$

Therefore,

$$
\lim _{s \rightarrow 0} \sup _{|h|_{H} \leq 1}\left\|\left[\left(f^{\prime} \circ(u+s h)-f^{\prime} \circ(u)\right) \cdot h\right]^{(k)}\right\|_{L^{2}(\mathcal{D})}=0, \quad r \leq k .
$$

This completes the proof that $F: H \rightarrow H$ is Fréchet differentiable. The fact that $F$ is $r$-times differentiable for $r \geq 2$ can be proved inductively using similar but lengthier computations. Details are left to the reader.

Using Itô's formula, it is easy to see that the solution of the following $H$-valued linear stochastic differential equation

$$
d u^{*}=B u^{*} d W(t), \quad u^{*}(0)=\psi \in H:=H_{0}^{k}(\mathcal{D})
$$

is given by

$$
u^{*}(t, \psi, \omega)(\xi):=Q(t, \xi, \omega) \psi(\xi), \quad \xi \in \mathcal{D}, \psi \in H, t \geq 0,
$$

where the process $Q: \mathbf{R}^{+} \times \mathcal{D} \times \Omega \rightarrow \mathbf{R}$ is defined by

$$
Q(t, \xi, \omega):=\exp \left\{\sum_{i=1}^{\infty} \sigma_{i}(\xi) W_{i}(t, \omega)-\frac{1}{2} \sum_{i=1}^{\infty} \sigma_{i}^{2}(\xi) t\right\}, t \geq 0, \xi \in \mathcal{D}, \omega \in \Omega .
$$

Using the perfect helix property of $(W, \theta)$, the reader may easily check the following cocycle identity for $Q$ :

$$
Q\left(t_{1}+t_{2}, \xi, \omega\right)=Q\left(t_{2}, \xi, \theta\left(t_{1}, \omega\right)\right) Q\left(t_{1}, \xi, \omega\right) \quad t_{1}, t_{2} \geq 0, \omega \in \Omega .
$$

The above identity immediately implies that $u^{*}: \mathbf{R}^{+} \times H \times \Omega \rightarrow H$ is a perfect linear cocycle with respect to the Brownian shift $\theta$.

We now prove the following proposition:

\section{Proposition 1.3.4.}

Assume $f \in C_{b}^{k}(R), k>\frac{d}{2}$, and the forgoing conditions on the coefficients of the spde (1.3.2). Let $S$ be a bounded subset of $H_{0}^{k}(\mathcal{D})$. Then for any $T>0$ and almost all $\omega \in \Omega$, the weak solution $u(t, \psi)$ of the spde (1.3.2) satisfies

$$
\sup _{\psi \in S} \sup _{0 \leq t \leq a}\|u(t, \psi)\|_{H_{0}^{k}(\mathcal{D})} \leq C(\omega, a)
$$

for any $a \in \mathbf{R}^{+}$, where $C(\omega, a)$ is a random positive constant. 
Proof.

Let $u(t, \psi)$ be the weak solution of the spde (1.3.2) with initial function $\psi \in H_{0}^{k}(\mathcal{D})$. Pick a sequence $\left\{\psi_{n}: n \geq 1\right\}$ of smooth functions in $C_{b}^{\infty}(\mathcal{D})$ such that $\psi_{n} \rightarrow \psi$ as $n \rightarrow \infty$ in $H_{0}^{k}(\mathcal{D})$. Let $u_{n}(t, \xi):=u\left(t, \psi_{n}\right)(\xi), t \geq 0, \xi \in \mathcal{D}, n \geq 1$. Then each $u_{n}, n \geq 1$, is a strong solution of the spde (1.3.2). Define $v_{n}(t, \xi):=Q(t, \xi)^{-1} u_{n}(t, \xi), t \geq 0, \xi \in \mathcal{D}$. Using the relations

$$
\begin{gathered}
d Q(t, \xi)=\sum_{i=1}^{\infty} \sigma_{i}(\xi) Q(t, \xi) d W_{i}(t), \quad t>0, \xi \in \mathcal{D} \\
d Q(t, \xi)^{-1}=\sum_{i=1}^{\infty} \sigma_{i}^{2}(\xi) Q(t, \xi)^{-1} d t-\sum_{i=1}^{\infty} \sigma_{i}(\xi) Q(t, \xi)^{-1} d W_{i}(t), \quad t>0, \xi \in \mathcal{D}
\end{gathered}
$$

and Itô's formula, it follows that

$$
\begin{aligned}
d v_{n}(t, \xi)= & Q(t, \xi)^{-1} \frac{1}{2} \Delta u_{n}(t, \xi) d t+Q(t, \xi)^{-1} f\left(u_{n}(t, \xi)\right) d t+Q(t, \xi)^{-1} u_{n}(t, \xi) \sum_{i=1}^{\infty} \sigma_{i}(\xi) d W_{i}(t) \\
& +u_{n}(t, \xi) Q(t, \xi)^{-1} \sum_{i=1}^{\infty} \sigma_{i}^{2}(\xi) d t-u_{n}(t, \xi) Q(t, \xi)^{-1} \sum_{i=1}^{\infty} \sigma_{i}(\xi) d W_{i}(t) \\
& -u_{n}(t, \xi) Q(t, \xi)^{-1} \sum_{i=1}^{\infty} \sigma_{i}^{2}(\xi) d t
\end{aligned}
$$

a.s. for all $t>0, \xi \in \mathcal{D}$,.

Therefore, for each $n \geq 1, v_{n}(t, \xi, \omega)$ satisfies the following parabolic equation with random coefficients:

$$
\begin{aligned}
\frac{\partial v_{n}}{\partial t}= & \frac{1}{2} \Delta v_{n}+<\nabla \ln Q(t, \xi), \nabla v_{n}>_{\mathbf{R}^{d}}-\left[\frac{1}{2} Q(t, \xi) \Delta Q(t, \xi)^{-1}\right. \\
& \left.\quad+<\nabla Q(t, \xi), \nabla Q(t, \xi)^{-1}>_{\mathbf{R}^{d}}\right] v_{n}+Q(t, \xi)^{-1} f\left(Q(t, \xi) v_{n}\right), \quad t>0, \\
v_{n}(0, \xi)= & \psi_{n}(\xi) .
\end{aligned}
$$

Let $v$ denote the unique weak solution of the parabolic random pde

$$
\begin{aligned}
\frac{\partial v}{\partial t}= & \frac{1}{2} \Delta v+<\nabla \ln Q(t, \xi), \nabla v>_{\mathbf{R}^{d}}-\left[\frac{1}{2} Q(t, \xi) \Delta Q(t, \xi)^{-1}\right. \\
& \left.+<\nabla Q(t, \xi), \nabla Q(t, \xi)^{-1}>_{\mathbf{R}^{d}}\right] v+Q(t, \xi)^{-1} f(Q(t, \xi) v), \quad t>0,
\end{aligned}
$$

for $t>0, \xi \in \mathcal{D}$, with $\psi \in H_{0}^{k}(\mathcal{D})$. Since the coefficients of (1.3.4) are smooth, it is well known that

$$
\lim _{n \rightarrow \infty} \sup _{0 \leq t \leq a}\left\|v(t, \cdot, \omega)-v_{n}(t, \cdot, \omega)\right\|_{H_{0}^{k}(\mathcal{D})}=0
$$

for each $\omega \in \Omega$ and any $a \in \mathbf{R}^{+}$. By rewriting (1.3.4), it is easy to see that $v$ satisfies the random pde

$$
\frac{\partial v}{\partial t}=\frac{1}{2} Q(t, \xi)^{-1} \Delta(Q(t, \xi) v)+Q(t, \xi)^{-1} f(Q(t, \xi) v), \quad t>0
$$


Since $\psi \in H_{0}^{k}(\mathcal{D}), k>\frac{d}{2}$, then by virtue of the Sobolev embedding of $H_{0}^{k}(\mathcal{D})$ into $L^{\infty}(\mathcal{D})$, we can view (1.3.4) as a random reaction diffusion equation in $L^{\infty}(\mathcal{D})$ whose non-linear term has linear growth and is globally Lipschitz. Now we use a standard argument to get an priori estimate for the solution of equation (1.3.4). Let $p(t, \eta, s, \xi)$ be the fundamental solution of the operator $\frac{1}{2} Q(t, \xi)^{-1} \Delta(Q(t, \xi) v)$, then it is well known that there are a positive constants $c_{1}, c_{2}$ such that:

$$
p(t, \eta, s, \xi) \leq c_{1}(t-s)^{-\frac{d}{2}} \exp \left\{-\frac{c_{2}|\eta-\xi|^{2}}{2(t-s)}\right\}
$$

for all $0 \leq s<t \leq a, \eta, \xi \in \mathcal{D}$ (see e.g. [Fri]). Therefore, it is easy to see that there is a positive constant $c$ such that $\int_{\mathcal{D}} p(t, \eta, s, \xi) d \eta \leq c$ for $0 \leq s<t \leq a, \xi \in \mathcal{D}$. By the classical variation of parameters formula, the solution of (1.3.4) satisfies the random integral equation

$$
v(t, \xi, \omega)=\int_{\mathcal{D}} p(t, \eta, 0, \xi) \psi(\eta) d \eta+\int_{0}^{t} \int_{\mathcal{D}} p(t, \eta, s, \xi) Q(s, \eta, \omega)^{-1} f(Q(s, \eta, \omega) v(s, \eta, \omega)) d \eta d s
$$

for $t>0, \xi \in \mathcal{D}, \psi \in H_{0}^{k}(\mathcal{D}), \omega \in \Omega$. From Lipschitz continuity of $f$, it is easy to see that

$$
\begin{aligned}
|v(t, \xi, \omega)| \leq & \|\psi\|_{\infty} \int_{\mathcal{D}} p(t, \eta, 0, \xi) d \eta+\int_{0}^{t} \int_{\mathcal{D}} p(t, \eta, s, \xi) Q(s, \eta, \omega)^{-1}|f(Q(s, \eta) v(s, \eta, \omega))-f(0)| d \eta d s \\
& +\int_{0}^{t} \int_{\mathcal{D}} p(t, \eta, s, \xi) Q(s, \eta, \omega)^{-1}|f(0)| d \eta d s \\
\leq & \left.C\|\psi\|_{\infty}+1\right)+L \int_{0}^{t} \int_{\mathcal{D}} p(t, \eta, s, \xi)|v(s, \eta, \omega)| d \eta d s \\
\leq & C_{1}\left(\|\psi\|_{\infty}+1\right)+L \int_{0}^{t} \sup _{\eta}|v(s, \eta, \omega)| \int_{\mathcal{D}} p(t, \eta, s, \xi) d \eta d s \\
\leq & C_{1}\left(\|\psi\|_{\infty}+1\right)+L \int_{0}^{t} \sup _{\eta}|v(s, \eta, \omega)| \int_{\mathcal{D}} p(t, \eta, s, \xi) d \eta d s \\
\leq & C_{2}\left(\|\psi\|_{\infty}+1\right)+C_{2} \int_{0}^{t}\|v(s, \cdot, \omega)\|_{\infty} d s
\end{aligned}
$$

for $0 \leq t \leq a, \omega \in \Omega$. In the above estimates, $L$ is the Lipschitz constant of $f$ and $C_{1}, C_{2}$ are positive constants. Hence, using Gronwall inequality, it follows that

$$
\sup _{t \in[0, a]}\|v(t, \cdot, \omega)\|_{\infty}<\infty
$$

for each $\omega \in \Omega$. Needless to say, this bound depends on $\omega$, but this does not affect our analysis here.

Now put $f \equiv 0$ in the random pde (1.3.5) and use uniqueness of solutions together with the identity

$$
Q\left(t_{1}+t_{2}, \xi, \omega\right)=Q\left(t_{2}, \xi, \theta\left(t_{1}, \omega\right)\right) Q\left(t_{1}, \xi, \omega\right) \quad t_{1}, t_{2} \geq 0, \omega \in \Omega
$$

in order to conclude that weak solutions of the linear spde

$$
d u(t, \xi)=\frac{1}{2} \Delta u(t, \xi) d t+B u(t, \xi) d W(t), \quad u(0, \xi)=\psi(\xi)
$$


yield a stochastic linear semiflow $\phi: \mathbf{R}^{+} \times H_{0}^{k}(\mathcal{D}) \times \Omega \rightarrow H_{0}^{k}(\mathcal{D})$ such that $(\phi, \theta)$ is a perfect $L\left(H_{0}^{k}(\mathcal{D})\right)$-valued cocycle. Full details of the argument are given in the proof of Theorem 1.4.1 in the next section.

It is easy to see that the weak solution $u$ of the spde (1.3.2) satisfies the following random integral equation:

$$
u(t, \xi, \omega)=\phi(t, \psi, \omega)(\xi)+\int_{0}^{t} \phi(t-s, \theta(s, \omega)) F(u(s, \xi, \omega)) d s,
$$

for $t \geq 0, \psi \in H_{0}^{k}(\mathcal{D}), \xi \in \mathcal{D}$.

Now, using Lemma 1.3.2 together with (1.3.6), one gets a positive random constant $C_{k}^{1}$ such that

$$
\|F(u(t, \cdot, \omega))\|_{H_{0}^{k}} \leq C_{k}^{1}(\omega, a)\|u(t, \cdot, \omega)\|_{H_{0}^{k}}
$$

for all $t \in[0, a], \omega \in \Omega$ and any $a \in \mathbf{R}^{+}$.

Finally, the assertion of the proposition follows from (1.3.8) and a simple application of Gronwall's lemma.

\section{Theorem 1.3.5.}

Suppose $k>\frac{d}{2}$. Assume $f: R \rightarrow R$ is a $C_{b}^{\infty}$ function. Assume all the forgoing conditions on the coefficients and the noise term in the spde (1.3.2). Then for each $\left.\psi \in H_{0}^{k}(\mathcal{D})\right)$ the spde (1.3.2) has a unique weak $\left(\mathcal{F}_{t}\right)_{t \geq 0}$-adapted solution $\left.u(\cdot, \psi, \cdot): \mathbf{R}^{+} \times \Omega \rightarrow H_{0}^{k}(\mathcal{D})\right)$. Furthermore, the family of weak solutions $u(\cdot, \psi, \cdot), \psi \in H_{0}^{k}(\mathcal{D})$, admits a $\left(\mathcal{B}\left(\mathbf{R}^{+}\right) \otimes \mathcal{B}\left(H_{0}^{k}(\mathcal{D})\right) \otimes \mathcal{F}, \mathcal{B}\left(H_{0}^{k}(\mathcal{D})\right)\right)$-measurable version $u: \mathbf{R}^{+} \times H_{0}^{k}(\mathcal{D}) \times \Omega \rightarrow H_{0}^{k}(\mathcal{D})$ having the following properties:

(i) For each $\psi \in H_{0}^{k}(\mathcal{D}), u(\cdot, \psi, \cdot): \mathbf{R}^{+} \times \Omega \rightarrow H_{0}^{k}(\mathcal{D})$ is $\left(\mathcal{F}_{t}\right)_{t \geq 0}$-adapted.

(ii) $(u, \theta)$ is a $C^{\infty}$ perfect cocycle on $H_{0}^{k}(\mathcal{D})$ (in the sense of Definition 1.1.2).

(iii) For each $(t, \omega) \in(0, \infty) \times \Omega$, the map $H_{0}^{k}(\mathcal{D}) \ni \psi \mapsto u(t, \psi, \omega) \in H_{0}^{k}(\mathcal{D})$ takes bounded sets into relatively compact sets.

(iv) For each $(t, \psi, \omega) \in(0, \infty) \times H_{0}^{k}(\mathcal{D}) \times \Omega$, and any integer $r \geq 1$, the Fréchet derivative $D^{(r)} u(t, \psi, \omega) \in L_{2}^{(r)}\left(H_{0}^{k}(\mathcal{D}), H_{0}^{k}(\mathcal{D})\right)$, and the map

$$
[0, \infty) \times H_{0}^{k}(\mathcal{D}) \times \Omega \ni(t, \psi, \omega) \mapsto D^{(r)} u(t, \psi, \omega) \in L^{(r)}\left(H_{0}^{k}(\mathcal{D}), H_{0}^{k}(\mathcal{D})\right)
$$

is strongly measurable.

(v) For any positive $a, \rho$ and any positive integer $r$,

$$
E \log ^{+}\left\{\sup _{\substack{0 \leq t_{1}, t_{2} \leq a \\ \psi \in H_{0}^{k}(\mathcal{D})}} \frac{\left\|u\left(t_{2}, \psi, \theta\left(t_{1}, \cdot\right)\right)\right\|_{H_{0}^{k}(\mathcal{D})}}{\left(1+\|\psi\|_{H_{0}^{k}(\mathcal{D})}\right)}\right\}<\infty
$$

and

$$
E \log ^{+} \sup _{\substack{0 \leq t_{1}, t_{2} \leq a \\\|\psi\|_{H_{0}^{k}(\mathcal{D})} \leq \rho}}\left\{\left\|D^{(r)} u\left(t_{2}, \psi, \theta\left(t_{1}, \cdot\right)\right)\right\|_{L^{(r)}\left(H_{0}^{k}(\mathcal{D}), H_{0}^{k}(\mathcal{D})\right)}\right\}<\infty
$$


Proof.

It is easy to see that the linear cocycle $(\phi, \theta)$ of the spde (1.3.7) in the proof of Proposition 1.3.4 satisfies all the assertions in Theorem 1.2.1 and Theorem 1.2.2. In view of Proposition 1.3.4, the theorem now follows by a straightforward modification of the proof of Theorem 1.2.6 (See Remark (i) following the proof of Theorem 1.2.6).

\subsection{Semilinear stochastic partial differential equations: Non-Lipschitz nonlinearity.}

In this section, we will study two types of semilinear stochastic partial differential equations with non-Lipschitz nonlinearities and infinite dimensional noise.

The two classes of spde's considered are stochastic reaction diffusion equations and stochastic Burgers equation with additive noise. We prove the existence of a compacting $C^{1}$-cocycle in each case.

(a) Stochastic reaction diffusion equations

This class of spde's has dissipative nonlinear terms and infinite dimensional spatially smooth white noise. We prove the existence of a compacting $C^{0,1}$-cocycle satisfying appropriate regularity properties (Theorem 1.4.1). It appears that the cocycle is in general not Fréchet differentiable over the space of all $L^{2}$ functions on the domain (cf. [Te], p. 298). However, for a subclass of dissipative non-linearities with a certain dimension requirement, we further prove that the cocycle is $C^{1}$ and possesses Oseledec-type integrability properties (Theorem 1.4.2).

In [F.2], Flandoli studied the existence of continuous semi-flows for a class of spde's with finite dimensional noise and polynomial nonlinearities of odd degree and with negative leading coefficients.

Consider the following stochastic reaction diffusion equation in a smooth bounded domain $\mathcal{D} \subset \mathbf{R}^{d}$

$$
\left.\begin{array}{rl}
d u & =\nu \Delta u d t+f(u(t)) d t+\sum_{i=1}^{\infty} \sigma_{i} u d W_{i}(t), \quad t>0 \\
u(0) & =\psi \\
\left.u(t)\right|_{\partial \mathcal{D}} & =0, \quad t>0 .
\end{array}\right\}
$$

where $\Delta$ is the Laplacian on $\mathcal{D}, \nu>0$ is a real constant. The initial function $\psi: \mathcal{D} \rightarrow \mathbf{R}$ is square-integrable with respect to Lebesgue measure on $\mathcal{D}$, and a Dirichlet boundary condition is assumed on the boundary $\partial \mathcal{D}$. The noise term $\sum_{i=1}^{\infty} \sigma_{i} u d W_{i}(t)$ is very similar to the one in (1.3.2) of section 1.3: We assume here that $\sigma_{i}: \mathcal{D} \rightarrow R, i \geq 1$, are functions in the Sobolev space $H_{0}^{s}(\mathcal{D})$ with $s>2+\frac{d}{2}$, and

$$
\sum_{i=1}^{\infty}\left\|\sigma_{i}\right\|_{H_{0}^{s}}^{2}<\infty .
$$

The nonlinearity $f: \mathbf{R} \rightarrow \mathbf{R}$ satisfies the following classical dissipativity conditions: 


\section{Conditions (D).}

The function $f$ is $C^{2}$, and there are positive constants $c_{i}, i=1,2,3,4$, and a positive integer $p$ such that

$$
\begin{aligned}
-c_{2}-c_{3} s^{2 p} \leq f(s) s & \leq c_{2}-c_{1} s^{2 p} \\
f^{\prime}(s) & \leq c_{4}
\end{aligned}
$$

for all $s \in \mathbf{R}$.

A typical example of a function $f: \mathbf{R} \rightarrow \mathbf{R}$ satisfying Conditions (D) is the polynomial $f(s):=\sum_{k=1}^{2 p-1} a_{k} s^{k}, s \in \mathbf{R}$, where $a_{2 p-1}<0$. (See e.g. [Te], pp. 83-85.)

Solutions of (1.4.1) are to be understood in a weak sense as defined below.

Consider the Hilbert space $H:=L^{2}(\mathcal{D})$ of all square-integrable functions $\psi: \mathcal{D} \rightarrow \mathbf{R}$ furnished with the $L^{2}$ inner product

$$
<\psi_{1}, \psi_{2}>_{H}:=\int_{\mathcal{D}} \psi_{1}(\xi) \psi_{2}(\xi) d \xi, \quad \psi_{1}, \psi_{2} \in H
$$

where $d \xi$ stands for Lebesgue measure on $\mathcal{D}$. Denote the induced norm on $H$ by

$$
|\psi|_{H}:=\left[\int_{\mathcal{D}}|\psi(\xi)|^{2} d \xi\right]^{1 / 2}, \quad \psi \in H
$$

Recall $C_{0}^{\infty}(\mathcal{D})$, the set of all smooth test functions $\phi: \mathcal{D} \rightarrow \mathbf{R}$ which vanish on $\partial \mathcal{D}$. Let $L^{\infty}(\mathcal{D})$ stand for all essentially bounded measurable functions $\psi: \mathcal{D} \rightarrow \mathbf{R}$ with the usual norm

$$
\|\psi\|_{\infty}:=\operatorname{essup}_{\xi \in \mathcal{D}}|\psi(\xi)|
$$

An $\left(\mathcal{F}_{t}\right)_{t \geq 0}$-adapted random field $u: \mathbf{R}^{+} \times \mathcal{D} \times \Omega \rightarrow \mathbf{R}$ is a weak solution of (1.4.1) if $u(t, \cdot, \omega) \in H$ for a.a. $\omega \in \Omega, t>0$, and the following identity holds:

$$
\left.\begin{array}{rl}
d<u(t), \phi>_{H} & =\nu<u(t), \Delta \phi>_{H} d t+<f(u(t)), \phi>_{H} d t+\sum_{i=1}^{\infty}<\sigma_{i} u(t), \phi>_{H} d W_{i}(t), \\
u(0) & =\psi \in L^{2}(\mathcal{D}), \\
\left.u(t)\right|_{\partial \mathcal{D}} & =0, \quad t>0,
\end{array}\right\}
$$

for all $\phi \in C_{0}^{\infty}(\mathcal{D})$ a.s.

Note that, unless $f$ has linear growth $(p=1$ in Conditions $(D))$, the Nemytskii operator $F(u)(\xi):=f(u(\xi)), \xi \in \mathcal{D}$, does not even map $H=L^{2}(\mathcal{D})$ into itself. Thus one cannot view (1.4.1) as a semilinear see on $H$. Nevertheless, we will show that for each $\psi \in H$, (1.4.1) admits a unique weak solution $u(t) \in H$ a.s., for all $t>0$. Furthermore, the ensemble of all weak solutions of (1.4.1) generates a a globally Lipschitz cocycle (also denoted by the same symbol) $u: \mathbf{R}^{+} \times H \times \Omega \rightarrow H$ satisfying the assertions of Theorem 1.4.1 below. In particular, the stochastic 
semiflow $u(t, \cdot, \omega): H \rightarrow H$ takes bounded sets into relatively compact sets in $H$, and its global Lipschitz constant has moments of all orders.

As for Fréchet differentiability of the cocycle $u: \mathbf{R}^{+} \times H \times \Omega \rightarrow H$ on the whole of $H$, it appears to be not true when $f$ is smooth and satisfies Conditions (D) (cf. [Te], p. 298). However, under a stronger dimension requirement on the polynomial growth rate $p$ of $f$, we are able to establish that the cocycle $u$ is $C^{1}$ on $H$ (Theorem 1.4.2). Furthermore, it satisfies similar assertions to those of Theorem 1.2.6. In particular, its Fréchet derivatives $D u(t, \psi, \omega): H \rightarrow H$ are compact for all $(t, \psi, \omega) \in(0, \infty) \times H \times \Omega$.

In (1.4.1), the special case $f(s):=s(1-s), s \in \mathbf{R}$, corresponds to the well-known stochastic $\mathrm{KPP}$ equation. It is not covered by the analysis in this section since it only admits positive solutions for all time. Its random travelling wave and ergodic properties were considered in [E-Z], [D-T-Z.1] and $[\mathrm{O}-\mathrm{V}-\mathrm{Z}]$. For the KPP equation with additive noise, the reader may refer to $[\mathrm{E}-\mathrm{H}]$ for the existence of the invariant measure.

The following lemma reduces (1.4.1) to a random family of reaction-diffusion equations.

\section{Lemma 1.4.1.}

Recall the process $Q: \mathbf{R}^{+} \times \mathcal{D} \times \Omega \rightarrow \mathbf{R}$ defined by $Q(t, \xi, \omega):=\exp \left\{\sum_{i=1}^{\infty} \sigma_{i}(\xi) W_{i}(t, \omega)-\right.$ $\left.\frac{1}{2} \sum_{i=1}^{\infty} \sigma_{i}^{2}(\xi) t\right\}, t \geq 0$. Let $u$ be a weak solution of (1.4.1) and $\operatorname{set} v(t, \xi, \omega):=Q(t, \xi, \omega)^{-1} u(t, \xi, \omega), t \geq$ 0. Define $\tilde{f}: \mathbf{R}^{+} \times \mathcal{D} \times \mathbf{R} \times \Omega \rightarrow \mathbf{R}$ by $\tilde{f}(t, \xi, s, \omega):=Q(t, \xi, \omega)^{-1} f(Q(t, \xi, \omega) s), t \in \mathbf{R}^{+}, \xi \in \mathcal{D}, s \in$ $\mathbf{R}, \omega \in \Omega$. Then $v$ is a weak solution of the random reaction-diffusion equation

$$
\left.\begin{array}{rl}
\frac{\partial v}{\partial t} & =\nu Q(t)^{-1} \Delta(Q(t) v)+\tilde{f}(t, v(t)), \quad t>0 \\
v(0) & =\psi \in L^{2}(\mathcal{D}) \\
t)\left.\right|_{\partial \mathcal{D}} & =0, \quad t>0 .
\end{array}\right\}
$$

Conversely, every weak solution $v$ of (1.4.2) corresponds to a weak solution $u$ of (1.4.1) given by $u(t, \xi, \omega):=Q(t, \xi, \omega) v(t, \xi, \omega), t \geq 0, \xi \in \mathcal{D}, \omega \in \Omega$.

Proof.

Suppose $u$ is a weak solution of (1.4.1) with initial function $\psi \in L^{2}(\mathcal{D})$. Define

$$
v(t, \xi, \omega):=Q(t, \xi, \omega)^{-1} u(t, \xi, \omega), t \geq 0, \xi \in \mathcal{D}, \omega \in \Omega
$$

Assume first that the initial function $\psi: \mathcal{D} \rightarrow \mathbf{R}$ is smooth. Then $u$ is a strong solution of (1.4.1). Hence by Itô's formula (as in the proof of Proposition 1.3.4), it follows that $v$ is a (strong) solution of the random reaction-diffusion equation (1.4.2). The case of a general $\psi \in L^{2}(\mathcal{D})$ can be handled by approximating $\psi$ in the $L^{2}$-norm by a sequence of smooth functions $\psi_{n}: \mathcal{D} \rightarrow \mathbf{R}, n \geq 1$, as in the proof of Proposition 1.3.4. 
A similar argument, using Itô's formula and the relation

$$
d Q(t, \xi)=\sum_{i=1}^{\infty} \sigma_{i}(\xi) Q(t, \xi) d W_{i}(t), \quad t>0,
$$

proves the second assertion of the lemma.

The next lemma shows that the non-linear term $\tilde{f}$ in (1.4.2) inherits the dissipativity properties of the original non-linear term $f$ in (1.4.1).

\section{Lemma 1.4.2.}

Suppose $f$ satisfies Conditions (D). Let $0<a<\infty$. Then there exist $\mathcal{F}$-measurable positive random variables $\tilde{c}_{i} \in \bigcap_{k=1}^{\infty} L^{k}(\Omega, \mathbf{R}), i=1,2,3$, such that the following is true:

$$
\left.\begin{array}{rl}
-\tilde{c}_{2}(\omega)-\tilde{c}_{3}(\omega) s^{2 p} \leq \tilde{f}(t, \xi, s, \omega) s & \leq-\tilde{c}_{1}(\omega) s^{2 p}+\tilde{c}_{2}(\omega), \\
\frac{\partial \tilde{f}(t, \xi, s, \omega)}{\partial s} & \leq c_{4}
\end{array}\right\}
$$

for all $t \in[0, a], s \in \mathbf{R}, \omega \in \Omega$.

Proof.

Fix $a \in(0, \infty), 0 \leq t \leq a, \xi \in \mathcal{D}, s \in \mathbf{R}, \omega \in \Omega$. Then Conditions (D) imply that

$$
\begin{aligned}
-c_{2} Q(t, \xi, \omega)^{-2}-c_{3} Q(t, \xi, \omega)^{(2 p-2)} s^{2 p} & \leq \tilde{f}(t, \xi, s, \omega) s \\
& \leq-c_{1} Q(t, \xi, \omega)^{(2 p-2)} s^{2 p}+c_{2} Q(t, \xi, \omega)^{-2}, \\
\frac{\partial \tilde{f}(t, s, \omega)}{\partial s} & \leq c_{4} .
\end{aligned}
$$

Define

$$
\begin{gathered}
\tilde{c}_{1}(\omega):=c_{1} \inf _{0 \leq t \leq a, \xi \in \mathcal{D}} Q(t, \xi, \omega)^{(2 p-2)}, \tilde{c}_{2}(\omega):=c_{2} \sup _{0 \leq t \leq a, \xi \in \mathcal{D}} Q(t, \xi, \omega)^{-2} \\
\tilde{c}_{3}(\omega):=c_{3} \sup _{0 \leq t \leq a, \xi \in \mathcal{D}} Q(t, \xi, \omega)^{(2 p-2)}
\end{gathered}
$$

for all $\omega \in \Omega$. By sample continuity of $Q(t, \xi)$ and $Q(t, \xi)^{-1}$, it is clear that each $\tilde{c}_{i}(\omega), i=1,2,3$, is finite for a.a. $\omega \in \Omega$. The estimates of the lemma follow immediately from the above inequalities and the definition of $Q(t, \xi)$. The existence of all moments of $\tilde{c}_{i}, i=1,2,3$, follows from BurkholderDavis-Gundy inequality and the fact that $Q(t, \xi)$ and $Q(t, \xi)^{-1}$ satisfy the linear sde's (1.4.3) and (1.4.4).

In view of Lemmas 1.4.1 and 1.4.2, we can now adapt standard methods from deterministic pde's in order to prove Theorem 1.4.1 below. In particular, the existence of the stochastic semiflow for weak solutions of the spde (1.4.1) follows from the regularity properties of solutions to the 
random reaction diffusion equation (1.4.2). For the existence of the semiflow of (1.4.2), its global Lipschitz continuity and compactness, we refer the reader to [Te], pp. 80-102, 371-374. Note that Lemma 1.4.2 ensures that the non-linear time-dependent random term $\tilde{f}$ in (1.4.2) satisfies appropriate dissipativity estimates which carry sufficient uniformity in $t$ to allow for the apriori estimates in $[\mathrm{Te}]$ to work. This renders the proof of Theorem 1.4.1 below an adaptation of the corresponding arguments in [Te]. Thus, we will only sketch the proof and leave many of the details to the reader.

\section{Theorem 1.4.1.}

Assume that $f$ in (1.4.1) satisfies Conditions (D). Then for each $\psi \in H:=L^{2}(\mathcal{D})$, the spde (1.4.1) admits a unique $\left(\mathcal{F}_{t}\right)_{t \geq 0}$-adapted weak solution $u(\cdot, \psi, \cdot): \mathbf{R}^{+} \times \Omega \rightarrow H$ such that $u(\cdot, \psi, \omega) \in L^{2 p}\left((0, T), L^{2 p}(\mathcal{D})\right) \cap C\left(\mathbf{R}^{+}, H\right)$ for a.a. $\omega \in \Omega$. The family of all weak solutions of (1.4.1) has a $\left(\mathcal{B}\left(\mathbf{R}^{+}\right) \otimes \mathcal{B}(H) \otimes \mathcal{F}, \mathcal{B}(H)\right)$-measurable version $u: \mathbf{R}^{+} \times H \times \Omega \rightarrow H$ with the following properties:

(i) For each $\psi \in H, u(\cdot, \psi, \cdot): \mathbf{R}^{+} \times \Omega \rightarrow H$ is an $\left(\mathcal{F}_{t}\right)_{t \geq 0}$-adapted weak solution of (1.4.1).

(ii) $(u, \theta)$ is a $C^{0,1}$ perfect cocycle on $H$ (in the sense of Definition 1.1.2).

(iii) For each $(t, \omega) \in(0, \infty) \times \Omega$, the map $H \ni \psi \mapsto u(t, \psi, \omega) \in H$ is globally Lipschitz and takes bounded sets in $H$ into relatively compact sets.

(iv) For any positive $a, \rho$,

$$
E \log ^{+} \sup _{\substack{0 \leq t \leq a \\|\psi|} \leq \rho}|u(t, \psi, \cdot)|_{H}<\infty
$$

(v) For each $\omega \in \Omega$,

$$
\limsup _{t \rightarrow \infty} \frac{1}{t} \log ^{+} \sup _{\substack{\psi_{1} \neq \psi_{2}, \psi_{1}, \psi_{2} \in H}} \frac{\left|u\left(t, \psi_{1}, \omega\right)-u\left(t, \psi_{2}, \omega\right)\right|_{H}}{\left|\psi_{1}-\psi_{2}\right|_{H}} \leq \frac{1}{2}\left(c_{4}-\nu \lambda_{1}-\sigma^{2}\right)
$$

where $\sigma^{2}:=\inf _{\xi \in \mathcal{D}} \sum_{i=1}^{\infty} \sigma_{i}^{2}(\xi)$. In particular, if

$$
\sup _{s \in \mathbf{R}} f^{\prime}(s)-\nu \lambda_{1}-\sigma^{2}<0
$$

then the stochastic flow $u(t, \cdot, \omega): H \rightarrow H$ is a uniform contraction for sufficiently large $t>0$.

\section{Proof.}

The existence and uniqueness of a weak solution of (1.4.1) follows from the corresponding result for the random reaction-diffusion equation (1.4.2) ([Te], pp. 89-91). Using the dissipativity estimates (1.4.5) on $\tilde{f}$, a straightforward modification of the Galerkin approximation technique in [Te] (pp. 89-91) gives the existence of a weak solution $u(\cdot, \psi, \omega): \mathbf{R}^{+} \rightarrow H$ of the random reaction-diffusion equation (1.4.2) for each fixed $\omega \in \Omega$ (cf. also [Ro], pp. 221-227). The joint 
measurability and $\left(\mathcal{F}_{t}\right)_{t \geq 0}$-adaptedness of the solution are also immediate consequences of the Galerkin approximations. This completes the proof of assertion (i) of the theorem.

To prove assertion (iii), denote by $v(\cdot, \psi, \cdot): \mathbf{R}^{+} \times \Omega \rightarrow H, \psi \in H$, the family of all weak solutions of the random pde (1.4.2). We will show that for each $\omega \in \Omega$, the map $H \ni \psi \mapsto$ $u(t, \psi, \omega) \in H$ is globally Lipschitz uniformly in $t$ over bounded sets in $\mathbf{R}^{+}$. To see this, let $\psi_{i} \in H, i=1,2$. Denote by $v_{i}(t):=v\left(t, \psi_{i}\right), t \geq 0, i=1,2$, the weak solutions of the random pde (1.4.2) starting at $\psi_{i} \in H, i=1,2$. Then multiplying both sides of the equation

$$
\frac{\partial\left(v_{1}(t)-v_{2}(t)\right)}{\partial t}=\nu Q(t)^{-1} \Delta\left(Q(t)\left(v_{1}(t)-v_{2}(t)\right)\right)+\tilde{f}\left(t, v_{1}(t)\right)-\tilde{f}\left(t, v_{2}(t)\right), \quad t>0,
$$

by $v_{1}(t)-v_{2}(t)$ and integrating over $\mathcal{D}$, we obtain

$$
\begin{aligned}
\int_{\mathcal{D}}\left(v_{1}(t)-v_{2}(t)\right) \frac{\partial\left(v_{1}(t)-v_{2}(t)\right)}{\partial t} d \xi=\nu< & Q(t)^{-1} \Delta\left(Q(t)\left(v_{1}(t)-v_{2}(t)\right)\right), v_{1}(t)-v_{2}(t)>_{H} \\
& +\int_{\mathcal{D}} \tilde{f}\left(t, v_{1}(t)\right)-\tilde{f}\left(t, v_{2}(t)\right)\left(v_{1}(t)-v_{2}(t)\right) d \xi,
\end{aligned}
$$

for all $t>0$. Using the Mean-Value Theorem and the second estimate in (1.4.5), it follows that

$$
\begin{aligned}
& \frac{1}{2} \frac{d}{d t}\left|v_{1}(t)-v_{2}(t)\right|_{H}^{2}= \nu<Q(t)^{-1} \Delta\left(Q(t)\left(v_{1}(t)-v_{2}(t)\right)\right), v_{1}(t)-v_{2}(t)>_{H} \\
& \quad+\int_{\mathcal{D}} \int_{0}^{1} \frac{\partial \tilde{f}}{\partial s}\left(t, \lambda v_{1}(t)+(1-\lambda) v_{2}(t)\right) d \lambda\left(v_{1}(t)-v_{2}(t)\right)^{2} d \xi \\
& \leq-\nu \lambda_{1}\left|v_{1}(t)-v_{2}(t)\right|_{H}^{2}+c_{4}\left|v_{1}(t)-v_{2}(t)\right|_{H}^{2}
\end{aligned}
$$

for all $t>0$. In the above inequality, $\lambda_{1}$ is the smallest eigenvalue of $-Q(t)^{-1} \Delta(Q(t) \cdot)$. This turns out to be the same as the smallest eigenvalue of $-\Delta$. Applying Gronwall's lemma to (1.4.6), we get

$$
\left|v_{1}(t, \omega)-v_{2}(t, \omega)\right|_{H}^{2} \leq\left|\psi_{1}-\psi_{2}\right|_{H}^{2} \exp \left\{\left(c_{4}-\nu \lambda_{1}\right) t\right\}
$$

for all $t \geq 0$ and all $\omega \in \Omega$. Using the relations $u\left(t, \psi_{i}, \omega\right)=Q(t, \xi, \omega) v_{i}(t, \omega), i=1,2$, in (1.4.7), we deduce that

$$
\left|u\left(t, \psi_{1}, \omega\right)-u\left(t, \psi_{2}, \omega\right)\right|_{H} \leq\left|\psi_{1}-\psi_{2}\right|_{H} \exp \left\{\frac{1}{2}\left(c_{4}-\nu \lambda_{1}\right) t\right\} \sup _{\xi \in \mathcal{D}} Q(t, \xi, \omega)
$$

for all $t \geq 0, \omega \in \Omega, \psi_{1}, \psi_{2} \in H$. For any $a>0$, define the random variable

$$
c_{5}(\omega):=\sup _{0 \leq t \leq a} \exp \left\{\frac{1}{2}\left(c_{4}-\nu \lambda_{1}\right) t\right\} \sup _{\xi \in \mathcal{D}} Q(t, \xi, \omega), \quad \omega \in \Omega .
$$

Then it is easy to see that $E \log ^{+} c_{5}<\infty$, and

$$
\left|u\left(t, \psi_{1}, \omega\right)-u\left(t, \psi_{2}, \omega\right)\right|_{H} \leq c_{5}(\omega)\left|\psi_{1}-\psi_{2}\right|_{H}
$$


for all $t \in[0, a], \omega \in \Omega, \psi_{1}, \psi_{2} \in H$. This proves the first assertion in (iii). (Note that (1.4.8) implies pathwise uniqueness of the weak solution to the spde (1.4.1): Just put $\psi_{1}=\psi_{2}=\psi$, a given initial function in $H$.) The local compactness of the semiflow $H \ni \psi \mapsto u(t, \psi, \omega) \in H, t>0, \omega$, follows from the fact that $H \ni \psi \mapsto v(t, \psi, \omega) \in H, t>0, \omega$, takes bounded sets in $H$ to relatively compact sets.

We next prove the perfect cocycle property in (ii). To this end, fix $\psi \in H, \omega \in \Omega, t_{1}, t_{2} \geq 0$. Define

$$
Y(t):=v\left(t+t_{1}, \psi, \omega\right), \quad Z(t):=Q\left(t_{1}, \omega\right)^{-1} v\left(t, Q\left(t_{1}, \omega\right) v\left(t_{1}, \psi, \omega\right), \theta\left(t_{1}, \omega\right)\right)
$$

for all $t \geq 0$. Recall the perfect cocycle identity:

$$
Q\left(t_{1}+t_{2}, \xi, \omega\right)=Q\left(t_{2}, \xi, \theta\left(t_{1}, \omega\right)\right) Q\left(t_{1}, \xi, \omega\right) \quad t_{1}, t_{2} \geq 0, \xi \in \mathcal{D} .
$$

By the definition of $\tilde{f}$ in Lemma 1.4.1 and the above cocycle property, one gets

$$
\begin{aligned}
\tilde{f}\left(t, \xi, s, \theta\left(t_{1}, \omega\right)\right) & =Q\left(t, \xi, \theta\left(t_{1}, \omega\right)\right)^{-1} f\left(Q\left(t, \xi, \theta\left(t_{1}, \omega\right)\right) s\right) \\
& =Q\left(t_{1}, \xi, \omega\right) Q\left(t+t_{1}, \xi, \omega\right)^{-1} f\left(Q\left(t+t_{1}, \xi, \omega\right) Q\left(t_{1}, \xi, \omega\right)^{-1} s\right) \\
& =Q\left(t_{1}, \xi, \omega\right) \tilde{f}\left(t+t_{1}, \xi, Q\left(t_{1}, \xi, \omega\right)^{-1} s, \omega\right)
\end{aligned}
$$

for all $s \in \mathbf{R}, t \geq 0, \xi \in \mathcal{D}$.

We now claim that the weak solution of the random reaction-diffusion equation (1.4.2) satisfies the following identity

$$
v\left(t+t_{1}, \psi, \omega\right)=Q\left(t_{1}, \omega\right)^{-1} v\left(t, Q\left(t_{1}, \omega\right) v\left(t_{1}, \psi, \omega\right), \theta\left(t_{1}, \omega\right)\right)
$$

for all $t \geq 1$. This says that $Y(t)=Z(t)$ for all $t \geq 0$. Using (1.4.11) and the relation between $u$ and $v$, it is easy to check that $(u, \theta)$ is a perfect cocycle. So we need only prove (1.4.11). By the definition of $Z$ and (1.4.10), it follows that

$$
\begin{aligned}
\frac{\partial Z}{\partial t}= & \nu Q\left(t_{1}, \omega\right)^{-1} Q\left(t, \theta\left(t_{1}, \omega\right)\right)^{-1} \Delta\left(Q\left(t, \theta\left(t_{1}, \omega\right)\right) v\left(t, Q\left(t_{1}, \omega\right) v\left(t_{1}, \psi, \omega\right), \theta\left(t_{1}, \omega\right)\right)\right) \\
& \quad+Q\left(t_{1}, \omega\right)^{-1} \tilde{f}\left(t, v\left(t, Q\left(t_{1}, \omega\right) v\left(t_{1}, \psi, \omega\right), \theta\left(t_{1}, \omega\right)\right), \theta\left(t_{1}, \omega\right)\right) \\
= & \nu Q\left(t+t_{1}, \omega\right)^{-1} \Delta\left(Q\left(t+t_{1}, \omega\right) Z(t)\right) \\
& \quad+\tilde{f}\left(t+t_{1}, Q\left(t_{1}, \omega\right)^{-1} v\left(t, Q\left(t_{1}, \omega\right) v\left(t_{1}, \psi, \omega\right), \theta\left(t_{1}, \omega\right)\right), \omega\right) \\
= & \nu Q\left(t+t_{1}, \omega\right)^{-1} \Delta\left(Q\left(t+t_{1}, \omega\right) Z(t)\right)+\tilde{f}\left(t+t_{1}, Z(t), \omega\right), \quad t>0
\end{aligned}
$$

and $Z(0)=v\left(t_{1}, \psi, \omega\right)$. Now from its definition, $Y$ also satisfies the same random pde:

$$
\frac{\partial Y}{\partial t}=\nu Q\left(t+t_{1}, \omega\right)^{-1} \Delta\left(Q\left(t+t_{1}, \omega\right) Y(t)\right)+\tilde{f}\left(t+t_{1}, Y(t), \omega\right), \quad t>0
$$

with the same initial condition $Y(0)=v\left(t_{1}, \psi, \omega\right)$. Therefore, by uniqueness of weak solutions to the above pde, we must have $Y(t)=Z(t)$ for all $t \geq 0$. This proves our claim, and hence $(u, \theta)$ is a perfect cocycle on $H$. 
Assertion (v) of the theorem follows easily from (1.4.8). This completes the proof of the theorem.

Our next result establishes Fréchet differentiability of the cocycle generated by the reaction diffusion equation:

$$
\left.\begin{array}{rl}
d u & =\nu \Delta u d t+\left(1-|u|^{\alpha}\right) u d t+\sum_{i=1}^{\infty} \sigma_{i}(\xi) u d W_{i}(t), \quad t>0 \\
u(0) & =\psi \in H:=L^{2}(\mathcal{D}) \\
\left.\right|_{\partial \mathcal{D}} & =0, \quad t>0 .
\end{array}\right\}
$$

where $\nu>0$ is a positive constant and $\Delta$ is the Laplacian on a smooth bounded domain $\mathcal{D}$ with Dirichlet boundary conditions. The result is established under the dimension requirement $\alpha<\frac{4}{d}$. It is not clear whether this condition is necessary for Fréchet differentiability of the cocycle.

\section{Theorem 1.4.2.}

In (1.4.12), assume that $\alpha<\frac{4}{d}$. Then for each $\psi \in H:=L^{2}(\mathcal{D})$, the spde (1.4.12) admits a unique $\left(\mathcal{F}_{t}\right)_{t \geq 0}$-adapted weak solution $u(\cdot, \psi, \cdot): \mathbf{R}^{+} \times \Omega \rightarrow H$ such that $u(\cdot, \psi, \omega) \in$ $L^{2 p}\left((0, T), L^{2 p}(\mathcal{D})\right) \cap C\left(\mathbf{R}^{+}, H\right)$ for a.a. $\omega \in \Omega$. The family of all weak solutions of (1.4.12) has a $\left(\mathcal{B}\left(R^{+}\right) \otimes \mathcal{B}(H) \otimes \mathcal{F}, \mathcal{B}(H)\right)$-measurable version $u: \mathbf{R}^{+} \times H \times \Omega \rightarrow H$ with the following properties:

(i) For each $\psi \in H, u(\cdot, \psi, \cdot): \mathbf{R}^{+} \times \Omega \rightarrow H$ is an $\left(\mathcal{F}_{t}\right)_{t \geq 0}$-adapted weak solution of (1.4.12).

(ii) $(u, \theta)$ is a $C^{1}$ perfect cocycle on $H$ (in the sense of Definition 1.1.2).

(iii) For each $(t, \omega) \in(0, \infty) \times \Omega$, the map $H \ni \psi \mapsto u(t, \psi, \omega) \in H$ is globally Lipschitz and takes bounded sets in $H$ into relatively compact sets.

(iv) For each $(t, \psi, \omega) \in(0, \infty) \times H \times \Omega$, the Fréchet derivative $D u(t, \psi, \omega) \in L(H)$ is compact, and the map

$$
[0, \infty) \times H \times \Omega \ni(t, \psi, \omega) \mapsto D u(t, \psi, \omega) \in L(H)
$$

is strongly measurable.

(v) For any positive $a, \rho$,

$$
E \log ^{+} \sup _{\substack{0 \leq t \leq a \\|\psi|} \leq \rho}\left\{|u(t, \psi, \cdot)|_{H}+\|D u(t, \psi, \cdot)\|_{L(H)}\right\}<\infty .
$$

Proof.

Fix any $\psi \in H=L^{2}(\mathcal{D})$. The existence and uniqueness of the solution to $(1.4 .12)$ in $L^{2}(\mathcal{D})$ is well-known as the nonlinear term satisfies the dissipativity condition ([D-Z.1]). This also follows by a similar argument to the proof of Theorem 1.4.1. So assertion (i) follows easily. The main purpose is to prove assertions (ii), (iii) and (iv). Recall that $Q(t, \xi):=\exp \left\{\sum_{i=1}^{\infty} \sigma_{i}(\xi) W_{i}(t)-\frac{1}{2} \sum_{i=1}^{\infty} \sigma_{i}(\xi)^{2} t\right\}, t \geq$ $0, \xi \in \mathcal{D}$, and let $v(t)=u(t) Q^{-1}(t), t \geq 0$. 
For simplicity of notation and till further notice, we will suppress the dependence of the random fields $u, v$, etc. on $\omega$.

Observe that $v(t, \psi)$ is a weak solution of the random reaction diffusion equation

$$
\frac{\partial v}{\partial t}=\nu \Delta v+2 \nu Q(t)^{-1} \nabla Q(t) \nabla v+v\left(\nu Q(t)^{-1} \Delta Q(t)+1-Q^{\alpha}(t)|v|^{\alpha}\right), \quad t>0 .
$$

By the Feynman-Kac formula, we have

$$
v(t, \psi)(\xi)=\hat{E}\left[\chi_{\tau_{t}=t} \psi\left(x_{t}\right) e^{\int_{0}^{t}\left(\nu Q\left(t-s, x_{s}\right)^{-1} \Delta Q\left(t-s, x_{s}\right)+1-Q^{\alpha}\left(t-s, x_{s}\right)|v|^{\alpha}(t-s, \psi)\left(x_{s}\right)\right) d s}\right]
$$

where $x$ is the solution of the following stochastic differential equation

$$
d x(s)=\sqrt{2 \nu} d B(s)+2 \nu \nabla \log Q\left(t-s, x_{s}\right) d s, \quad x_{0}=\xi \in \mathcal{D},
$$

and $B$ is a Brownian motion in $\mathbf{R}^{d}$ independent of the $W_{i}, i \geq 1$. In (1.4.14), $\tau_{t}:=\min (\tau, t)$, where $\tau$ is the first time the diffusion $x$ hits $\partial \mathcal{D}$. Define $\beta:=\nu \sup _{0 \leq s \leq t \leq a, \xi \in \mathcal{D}} \frac{\Delta Q(t-s, \xi)}{Q(t-s, \xi)}$ for any $a>0$. It follows from Jensen's inequality and (1.4.14) that

$$
\begin{aligned}
|v(t, \psi)|_{H}^{2} & \leq \int_{\mathcal{D}}\left(\hat{E} \chi_{\tau_{t}=t}\left|\psi\left(x_{t}\right)\right| e^{(\beta+1) t}\right)^{2} d \xi \\
& \leq e^{2(\beta+1) t} \int_{\mathcal{D}}\left(\int_{\mathcal{D}} p(t, \xi, y)|\psi(y)| d y\right)^{2} d \xi \\
& \leq e^{2(\beta+1) t} \int_{\mathcal{D}} \int_{\mathcal{D}} p(t, \xi, y)(\psi(y))^{2} d y d \xi \\
& \leq e^{2(\beta+1) t}|\psi|_{H}^{2}, \quad 0 \leq t \leq a .
\end{aligned}
$$

In the above inequalities, $p(t, \xi, y)$ denotes the heat kernel associated with $\nu \Delta+2 \nu(\nabla \log Q(t)) \nabla$ on $\mathcal{D}$ with Dirichlet boundary condition. Define the induced heat semigroup $T_{t}: H \rightarrow H, t \geq 0$, by

$$
\left(T_{t} \psi\right)(\xi):=\int_{\mathcal{D}} p(t, \xi, y) \psi(y) d y, \quad \psi \in H, \xi \in \mathcal{D}, t \geq 0 .
$$

Note that there exists a constant $c>0$ such that

$$
p(t, \xi, y) \leq \frac{c}{t^{\frac{d}{2}}}, \quad \xi, y \in \mathcal{D}, t>0 .
$$

It is easy to see, using Jensen's inequality and (1.4.16), that

$$
\begin{aligned}
|v(t, \psi)(\xi)|^{2} & \leq\left(\hat{E} \chi_{\tau_{t}=t}\left|\psi\left(x_{t}\right)\right| e^{(\beta+1) t}\right)^{2} \\
& \leq e^{2(\beta+1) t} \hat{E} \chi_{\tau_{t}=t}\left(\psi\left(x_{t}\right)\right)^{2} \\
& \leq e^{2(\beta+1) t} \int_{\mathcal{D}} p(t, \xi, y)(\psi(y))^{2} d y \\
& \leq e^{2(\beta+1) t} \frac{c}{t^{\frac{d}{2}}} \int_{\mathcal{D}} \psi^{2}(y) d y
\end{aligned}
$$


for all $\xi \in \mathcal{D}$ and $t>0$. Hence

$$
\|v(t, \psi)\|_{\infty} \leq \frac{\sqrt{c} e^{(\beta+1) t}}{t^{\frac{d}{4}}}|\psi|_{H}
$$

for $t>0$.

Now let $\psi, g \in L^{2}(\mathcal{D})$ with $|g|_{H} \leq 1$, and h be a small real number. Since $v$ is a mild solution of (1.4.13), it follows that $v(t, \psi+h g)-v(t, \psi)$ satisfies the following convolution equation in $H$ :

$$
\begin{aligned}
v(t, \psi+h g)-v(t, \psi)= & h T_{t} g+\int_{0}^{t} T_{t-s}(v(s, \psi+h g)-v(s, \psi))\left(1+\frac{\Delta Q(s)}{Q(s)}\right) d s \\
& +\int_{0}^{t} T_{t-s} Q^{\alpha}(s)\left[v(s, \psi)|v(s, \psi)|^{\alpha}-v(s, \psi+h g)|v(s, \psi+h g)|^{\alpha}\right] d s, t>0 .
\end{aligned}
$$

Define $m(x):=x|x|^{\alpha}$ for each $x \in \mathbf{R}$. Then $m^{\prime}(x)=(\alpha+1)|x|^{\alpha}, x \in \mathbf{R}$. By the Mean-Value Theorem, we have

$$
\begin{aligned}
v(s, \psi+h g)|v(s, \psi+h g)|^{\alpha}-v(s, \psi)|v(s, \psi)|^{\alpha} \\
=(\alpha+1) \int_{0}^{1}|r v(s, \psi+h g)+(1-r) v(s, \psi)|^{\alpha} d r(v(s, \psi+h g)-v(s, \psi))
\end{aligned}
$$

for all $s \in \mathbf{R}^{+}$. Combining (1.4.18) and (1.4.19) we obtain

$$
\begin{aligned}
& \quad|v(t, \psi+h g)-v(t, \psi)|_{H} \\
& \leq h|g|_{H}+c_{1}(\omega) \int_{0}^{t}|v(s, \psi+h g)-v(s, \psi)|_{H} d s \\
& \quad+c_{1}(\omega)(\alpha+1) \int_{0}^{t}\left(\|v(s, \psi+h g)\|_{L^{\infty}}+\|v(s, \psi)\|_{L^{\infty}}\right)^{\alpha}|v(s, \psi+h g)-v(s, \psi)|_{H} d s,
\end{aligned}
$$

for all $0 \leq t \leq a$, where $c_{1}(\omega)$ is a positive random constant. By virtue of (1.4.17), we get

$$
\begin{aligned}
|v(t, \psi+h g)-v(t, \psi)|_{H} \leq & h|g|_{H}+c_{1}(\omega) \int_{0}^{t}|v(s, \psi+h g)-v(s, \psi)|_{H} d s \\
& +c_{2}(\omega)(\alpha+1) \int_{0}^{t} \frac{1}{s^{\frac{d}{4} \alpha}}|v(s, \psi+h g)-v(s, \psi)|_{H} d s
\end{aligned}
$$

for all $0 \leq t \leq a$, where $c_{2}(\omega)>0$ is a random constant depending on the ball $\left\{g \in H:|g|_{H} \leq 1\right\}$. Using Gronwall's lemma and the requirement $\alpha d<4$, we obtain

$$
\sup _{0 \leq t \leq a} \sup _{\substack{g \in H \\|g|_{H} \leq 1}}|v(t, \psi+h g)-v(t, \psi)|_{H} \leq C(\omega) h
$$

Consider the $L(H)$-valued integral equation:

$$
G_{t}(\psi)=T_{t}+\int_{0}^{t} T_{t-s}\left(Q(s)^{-1} \Delta Q(s)+1-(\alpha+1) Q^{\alpha}(s)|v(s, \psi)|^{\alpha}\right) G_{s}(\psi) d s, t \geq 0
$$


where $1-(\alpha+1) Q^{\alpha}(s)|v(s, \psi)|^{\alpha}$ is regarded as a multiplication operator on $L^{2}(\mathcal{D})$ whose operator norm satisfies the inequality

$$
\left\|Q(s)^{-1} \Delta Q(s)+1-(\alpha+1) Q^{\alpha}(s)|v(s, \psi)|^{\alpha}\right\|_{\infty} \leq C_{a}(\omega) \frac{1}{s^{\frac{d}{4} \alpha}}|\psi|_{H}^{\alpha}, \quad 0<s \leq a,
$$

for a positive random constant $C_{a}(\omega)$.

Claim: There exists a unique, continuous solution $[0, \infty) \ni t \mapsto G_{t}(\psi) \in L(H)$ to equation (1.4.21). Moreover for $t>0, G_{t}(\psi): H \rightarrow H$ is compact.

Proof of claim: Let $G_{t}^{1}(\psi)=T_{t}, t \geq 0$. Define the sequence $\left\{G_{t}^{n}(\psi)\right\}_{n=1}^{\infty}$ inductively by

$$
G_{t}^{n+1}(\psi)=T_{t}+\int_{0}^{t} T_{t-s}\left(Q(s)^{-1} \Delta Q(s)+1-(\alpha+1) Q^{\alpha}(s)|v(s, \psi)|^{\alpha}\right) G_{s}^{n}(\psi) d s
$$

for $n \geq 1$. Then (1.4.22) and (1.4.23) imply that

$$
\left\|G_{t}^{n+1}(\psi)\right\|_{L(H)} \leq 1+C_{a}(\omega)\|\psi\|^{\alpha} \int_{0}^{t} \frac{1}{s^{\frac{d}{4} \alpha}}\left\|G_{s}^{n}(\psi)\right\|_{L(H)} d s ., \quad 0<t \leq a
$$

Since $\frac{\alpha d}{4}<1$, then by the standard successive approximation technique it follows that the sequence $\left\{G^{n}(\psi)\right\}_{n=1}^{\infty}$ converges to the unique solution of (1.4.12). Next we prove that $G_{t}(\psi)$ is compact for each $t>0$. It suffices to show that a Cauchy sequence can be extracted from the set $\left\{G_{t}(\psi)(g)\right.$ : $\left.|g|_{H} \leq 1\right\}$ for each $t>0$. Let $\delta_{m}, m \geq 1$ be a sequence of positive numbers decreasing to zero. Since $T_{t}$ is compact for every $t>0$, by a diagonal process there exists a sequence $g_{n} \in H$ with $\left|g_{n}\right|_{H} \leq 1$ such that $T_{\delta_{m}} g_{n}, n \geq 1$ is a Cauchy sequence for every $m$. Since $T_{t}, t \geq 0$, is a contraction semigroup on $H$, it is easy to see that $T_{t} g_{n}, n \geq 1$ is a Cauchy sequence for every $t>0$. Now consider

$$
\begin{aligned}
& G_{t}(\psi)\left(g_{n}\right)-G_{t}(\psi)\left(g_{m}\right)=T_{t}\left(g_{n}-g_{m}\right) \\
& +\int_{0}^{t} T_{t-s}\left(Q(s)^{-1} \Delta Q(s)+1-(\alpha+1) Q^{\alpha}(s)|v(s, \psi)|^{\alpha}\right)\left(G_{s}(\psi)\left(g_{n}\right)-G_{s}(\psi)\left(g_{m}\right)\right) d s, t \geq 0 .
\end{aligned}
$$

Hence,

$$
\begin{aligned}
& \left|G_{t}(\psi)\left(g_{n}\right)-G_{t}(\psi)\left(g_{m}\right)\right|_{H} \\
& \quad \leq\left|T_{t} g_{n}-T_{t} g_{m}\right|_{H}+C_{a}(\omega)|\psi|_{H}^{\alpha} \int_{0}^{t} \frac{1}{s^{\frac{d}{4} \alpha}}\left|G_{s}(\psi)\left(g_{n}\right)-G_{s}(\psi)\left(g_{m}\right)\right|_{H} d s .
\end{aligned}
$$

for all $t \in[0, a]$. Set $l(t):=\limsup _{n, m \rightarrow \infty}\left|G_{t}(\psi)\left(g_{n}\right)-G_{t}(\psi)\left(g_{m}\right)\right|_{H}, 0 \leq t \leq a$. Taking $\limsup _{n, m \rightarrow \infty}$ on both sides of (1.4.26) we obtain

$$
l(t) \leq C_{a}(\omega)|\psi|_{H}^{\alpha} \int_{0}^{t} \frac{1}{s^{\frac{d}{4} \alpha}} l(s) d s, \quad 0<t \leq a
$$

This implies that $l(t)=0$ for all $t>0$, and completes the proof of the claim. 
Next we show that $v$ is Fréchet differentiable and $D v(t, \psi)=G_{t}(\psi)$ for all $t \geq 0$. First we note that by using the Feynman-Kac formula and a similar argument as in the proof of (1.4.15) and (1.4.17), one has

$$
\int_{\mathcal{D}} G_{t}(\psi)(g)(\xi) d \xi \leq e^{2(\beta+1) t}|g|_{H}^{2}
$$

and

$$
G_{t}(\psi)(g)(\xi) \leq \frac{\sqrt{c} e^{(\beta+1) t}}{t^{\frac{d}{4}}}|g|_{H}^{2}, \quad 0<t \leq a
$$

Denote

$$
\mu_{t}(\psi, g)=\frac{1}{h}(v(t, \psi+h g)-v(t, \psi))-G_{t}(\psi)(g), \quad t>0 .
$$

It is easy to see that $\mu$ satisfies the following integral equation:

$$
\begin{aligned}
\mu_{t}(\psi, g)= & \int_{0}^{t} T_{t-s}\left(1+\frac{\Delta Q(s)}{Q(s)}\right) \mu_{s}(\psi, g) d s \\
& -\int_{0}^{t} T_{t-s} Q^{\alpha}(s)\left[\frac{1}{h}\left(v(s, \psi+h g)|v(s, \psi+h g)|^{\alpha}-v(s, \psi)|v(s, \psi)|^{\alpha}\right)\right] d s \\
& +(\alpha+1) \int_{0}^{t} T_{t-s} Q^{\alpha}(s)\left[|v(s, \psi)|^{\alpha} G_{s}(\psi)(g)\right] d s, \quad t \geq 0 .
\end{aligned}
$$

Using $m(y)-m(x)=\int_{0}^{1} m^{\prime}(r y+(1-r) x) d r(y-x)$ it follows from (1.4.30) that

$$
\begin{aligned}
& \mu_{t}(\psi, g)= \int_{0}^{t} T_{t-s}\left(1+\frac{\Delta Q(s)}{Q(s)}\right) \mu_{s}(\psi, g) d s \\
&-(\alpha+1) \int_{0}^{t} T_{t-s} Q^{\alpha}(s)\left[\int_{0}^{1}|r v(s, \psi+h g)+(1-r) v(s, \psi)|^{\alpha} d r \mu_{s}(\psi, g)\right] d s \\
&+(\alpha+1) \int_{0}^{t} T_{t-s} Q^{\alpha}(s)\left[\int_{0}^{1}\left(|v(s, \psi)|^{\alpha}-|r v(s, \psi+h g)+(1-r) v(s, \psi)|^{\alpha}\right) d r\right. \\
&\left.G_{s}(\psi)(g)\right] d s, \quad t \geq 0 .
\end{aligned}
$$

Set $D(t):=\sup _{|g|_{H} \leq 1}\left|\mu_{t}(\psi, g)\right|_{H}, t \geq 0$. Using the $L^{\infty}$ bound on $v(s, \psi+h g)$ this implies that for $0<t \leq a$, one has

$$
\begin{aligned}
D(t) \leq & C(\omega) \int_{0}^{t} D(s) d s+C(\omega) \int_{0}^{t} \frac{1}{s^{\frac{d}{4} \alpha}} D(s) d s \\
& +C(\omega) \sup _{|g|_{H} \leq 1} \int_{0}^{t} \mid \int_{0}^{1}\left(|v(s, \psi)|^{\alpha}\right. \\
& \left.\quad-|r v(s, \psi+h g)+(1-r) v(s, \psi)|^{\alpha}\right)\left.d r G_{s}(\psi)(g)\right|_{H} d s .
\end{aligned}
$$

Again by Gronwall's lemma, it follows that there is a random constant $C(\omega)$ such that

$$
\begin{aligned}
& D(t) \\
& \leq C(\omega) \sup _{|g|_{H} \leq 1} \int_{0}^{t}\left|\left(\int_{0}^{1}\left(|v(s, \psi)|^{\alpha}-|r v(s, \psi+h g)+(1-r) v(s, \psi)|^{\alpha}\right) d r\right) G_{s}(\psi)(g)\right|_{H} d s .
\end{aligned}
$$


for all $t \in[0, a]$. To complete the proof of assertions (ii) and (iv), it suffices to show that

$$
\lim _{h \rightarrow 0} \sup _{|g|_{H} \leq 1} \int_{0}^{t}\left|\left(\int_{0}^{1}\left(|v(s, \psi)|^{\alpha}-|r v(s, \psi+h g)+(1-r) v(s, \psi)|^{\alpha}\right) d r\right) G_{s}(\psi)(g)\right|_{H} d s=0
$$

for all $t \in[0, a]$. Let us prove (1.4.24) for $\alpha \leq 1$ and $\alpha>1$ separately. Assume first $\alpha \leq 1$. By Hölder inequality,

$$
\begin{aligned}
& \sup _{|g|_{H} \leq 1} \int_{0}^{t}\left|\left(\int_{0}^{1}\left(|v(s, \psi)|^{\alpha}-|r v(s, \psi+h g)+(1-r) v(s, \psi)|^{\alpha}\right) d r\right) G_{s}(\psi)(g)\right|_{H} d s \\
\leq & \sup _{|g|_{H} \leq 1} \int_{0}^{t}\left|\left(|v(s, \psi)-v(s, \psi+h g)|^{\alpha}\right) G_{s}(\psi)(g)\right|_{H} d s \\
\leq & \sup _{|g|_{H} \leq 1} \int_{0}^{t}\left\|G_{s}(\psi)(g)\right\|_{L^{\infty}}^{\alpha}\left(|v(s, \psi)-v(s, \psi+h g)|_{H}^{\alpha}\right)\left|G_{s}(\psi)(g)\right|^{1-\alpha} \| d s \\
\leq & \sup _{|g|_{H} \leq 1} \int_{0}^{t}\left\|G_{s}(\psi)(g)\right\|_{L^{\infty}}^{\alpha}|v(s, \psi)-v(s, \psi+h g)|_{H}^{\alpha}\left|G_{s}(\psi)(g)\right|_{H}^{1-\alpha} d s .
\end{aligned}
$$

By virtue of (1.4.20) and (1.4.28), we get

$$
\begin{gathered}
\sup _{|g|_{H} \leq 1} \int_{0}^{t}\left|\left(\int_{0}^{1}\left(|v(s, \psi)|^{\alpha}-|r v(s, \psi+h g)+(1-r) v(s, \psi)|^{\alpha}\right) d r\right) G_{s}(\psi)(g)\right| \mid d s \\
\quad \leq C_{\alpha}(\omega) h^{\alpha} \int_{0}^{t} \frac{1}{s^{\frac{d}{4} \alpha}} d s=C_{\alpha}(\omega) \frac{1}{1-\frac{\alpha d}{4}} t^{1-\frac{\alpha d}{4}} h^{\alpha}, \quad 0<t \leq a,
\end{gathered}
$$

where $C_{\alpha}(\omega)$ is a random constant depending on the set $\left\{g \in L^{2}(\mathcal{D}):\|g\|_{L^{2}} \leq 1\right\}$. This implies (1.4.24).

Assume now that $\alpha>1$. Then

$$
\begin{aligned}
& \int_{0}^{t}\left|\left(\int_{0}^{1}\left(|v(s, \psi)|^{\alpha}-|r v(s, \psi+h g)+(1-r) v(s, \psi)|^{\alpha}\right) d r\right) G_{s}(\psi)(g)\right|_{H} d s \\
\leq & \int_{0}^{t} d s \int_{0}^{1} d r\left|\left(|v(s, \psi)|^{\alpha}-|r v(s, \psi+h g)+(1-r) v(s, \psi)|^{\alpha}\right) G_{s}(\psi)(g)\right|_{H} \\
\leq & \int_{0}^{t} d s \int_{0}^{1} d r \int_{0}^{1} d k \alpha \mid(k|v(s, \psi)|+(1-k)|r v(s, \psi+h g)+(1-r) v(s, \psi)|)^{\alpha-1} \\
& \left.|v(s, \psi+h g)-v(s, \psi)| G_{s}(\psi)(g)\right|_{H} \\
\leq & \int_{0}^{t} d s \int_{0}^{1} d r \int_{0}^{1} d k \alpha\left\|(k|v(s, \psi)|+(1-k)|r v(s, \psi+h g)+(1-r) v(s, \psi)|)^{\alpha-1}\right\|_{L^{\infty}} \\
& \left.|| G_{s}(\psi)(g)\right|_{L^{\infty}}|v(s, \psi+h g)-v(s, \psi)|_{H}, \quad 0<t \leq a .
\end{aligned}
$$

By (1.4.17), (1.4.20) and (1.4.28) it follows from (1.4.35) that

$$
\sup _{|g|_{H} \leq 1} \int_{0}^{t}\left|\left(\int_{0}^{1}\left(|v(s, \psi)|^{\alpha}-|r v(s, \psi+h g)+(1-r) v(s, \psi)|^{\alpha}\right) d r\right) G_{s}(\psi)(g)\right|_{H} d s
$$




$$
\leq C_{\alpha}(\omega) h \int_{0}^{t} \frac{1}{s^{\frac{d}{4}(\alpha-1)}} \frac{1}{s^{\frac{d}{4}}} d s, 0<t \leq a
$$

This implies (1.4.34). So assertion (iv) holds.

To establish (iii), use (1.4.18), (1.4.19) and a similar argument to the proof of (1.4.20), to obtain the following inequality

$$
\begin{aligned}
\left|v\left(t, \psi_{m}\right)-v\left(t, \psi_{n}\right)\right|_{H} \leq & \left|T_{t} \psi_{m}-T_{t} \psi_{n}\right|_{H}+C_{1}(\omega) \int_{0}^{t}\left|v\left(s, \psi_{m}\right)-v\left(s, \psi_{n}\right)\right|_{H} d s \\
& +C_{2}(\omega)(\alpha+1) \int_{0}^{t} \frac{1}{s^{\frac{d}{4} \alpha}}\left|v\left(s, \psi_{m}\right)-v\left(s, \psi_{n}\right)\right|_{H} d s, \quad 0<t \leq a,
\end{aligned}
$$

for $\psi_{n}, \psi_{m} \in H$ such that $\left|\psi_{m}\right|_{H},\left|\psi_{n}\right|_{H} \leq 1$. As in the proof of the compactness of $D v(t, \psi)$, we can select a subsequence denoted also by $\left\{\psi_{n}\right\} \subset\left\{\psi:|\psi|_{H} \leq 1\right\}$ such that for each $t>0, \mid T_{t} \psi_{n}-$ $\left.T_{t} \psi_{m}\right|_{H} \rightarrow 0$ as $n, m \rightarrow \infty$. One then can prove from (1.4.32) that $\lim _{n, m \rightarrow \infty}\left|v\left(t, \psi_{n}\right)-v\left(t, \psi_{m}\right)\right|_{H}=0$. Therefore $v(t, \cdot): H \rightarrow H$ is compact or each $t>0$. This implies the compactness of each Fréchet derivative $D u(t, \psi, \omega): H \rightarrow H, t>0, \omega \in \Omega$. Hence the first assertion in (iv) holds.

To prove the strong measurability assertion in (iv), we now highlight the dependence of $u$ on $\omega$. Note first that the map

$$
[0, \infty) \times H \times \Omega \ni(t, \psi, \omega) \mapsto u(t, \psi, \omega) \in H
$$

is jointly measurable. This is a consequence of the (uniform) continuity of

$$
[0, a] \times H \ni(t, \psi) \mapsto u(t, \psi, \omega) \in H, \quad \omega \in \Omega,
$$

and the measurability of

$$
\Omega \ni \omega \mapsto u(t, \psi, \omega) \in H, \quad(t, \psi) \in \mathbf{R}^{+} \times H .
$$

Secondly, the joint strong measurability of

$$
[0, \infty) \times H \times \Omega \ni(t, \psi, \omega) \mapsto D u(t, \psi, \omega) \in L(H)
$$

follows from the relation

$$
D u(t, \psi, \omega)(\eta)=\lim _{h \rightarrow 0} \frac{1}{h}[u(t, \psi+h \eta, \omega)-u(t, \psi, \omega)], \quad(t, \omega) \in \mathbf{R}^{+} \times \Omega, \psi, \eta \in H .
$$

Finally, note that the integrability estimate in (v) follows from the Lipschitz property of $u(t, \cdot, \omega)$ : $H \rightarrow H,(t, \omega) \in \mathbf{R}^{+} \times \Omega$. In particular, (1.4.38) and the above Lipschitz property give

$$
\|D u(t, \psi, \omega)\|_{L(H)} \leq c_{5}(\omega)
$$

for all $(t, \psi, \omega) \in[0, a] \times H \times \Omega$, with $E \log ^{+} c_{5}<\infty$.

Remarks. 
(i) It is easy to see that above proof is also valid for the initial boundary value problem with Neumann boundary condition. Note the exact formula of the heat kernel was not needed in the proof. Only estimates such as (1.4.6) and (1.4.7) were actually needed. These kind of estimate holds for Laplacian operator on a bounded domain with smooth boundary and Neumann boundary condition. The generalized solution of (1.4.1) can be defined following Freidlin $[\mathrm{Fr}]$ :

$$
\begin{aligned}
& u(t, \psi)(\xi) \\
& \quad=\hat{E}\left[\psi\left(x_{t}^{*}\right) e^{\int_{0}^{t}\left(\frac{\Delta\left(t-s, x_{s}^{*}\right)}{Q\left(t-s, x_{s}^{*}\right)}+1-|u|^{\alpha}(t-s, \psi)\left(x_{s}^{*}\right)\right) d s-\frac{1}{2} \sum_{i=1}^{\infty} \int_{0}^{t} \sigma_{i}^{2}\left(x_{s}^{*}\right) d s+\sum_{i=1}^{\infty} \sigma_{i}\left(x_{s}^{*}\right) d W_{i}(t-s)}\right]
\end{aligned}
$$

a.s. Here $x_{t}^{*}$ is a diffusion process starting at $\xi \in \mathcal{D}$ with reflection on the boundary $\partial \mathcal{D}$ generated with the operator $\nu \Delta+2 \nu \nabla \log Q(t) \nabla$. One can see that the analysis in the proof of Theorem 1.4.1 carries through for this case as well.

(ii) The dimension restriction is used only to guarantee the Fréchet differentiability of the semiflow in Theorem 1.4.2. This condition is not needed for the existence of the globally Lipschitz flow in Theorem 1.4.1. The conditions in Theorem 1.4.2 are stronger than those in Theorem 1.4.1, and accordingly the result.

(b) Burgers equation with additive noise

The stochastic Burgers equation has been considered intensively by many researchers in recent years ([B-C-J], [B-C-F], [D-T-Z], [D-Z.2], [D-D-T], [E-V], [H-L-O-U-Z], [Si], [T-Za], [T-Z]). Here we consider the following stochastic Burgers equation on the interval $[0,1]$,

$$
\begin{aligned}
d u+u \frac{\partial u}{\partial \xi} d t & =\nu \Delta u d t+d W(t), \quad t>0 \\
u(0, \psi)(\xi) & =\psi(\xi) \\
u(t, \psi)(0) & =u(t, \psi)(1)=0
\end{aligned}
$$

where the viscosity $\nu$ is a positive constant. Here $W$ is an infinite dimensional Brownian motion in $L^{2}[0,1]$ on a probability space $(\Omega, \mathcal{F}, P)$ :

$$
W(t):=\sum_{k=1}^{\infty} \sqrt{\lambda_{k}} e_{k} W^{k}(t) .
$$

In (1.4.40), each $e_{k}$ is an eigenfunction of $-\nu \Delta$ associated with its eigenvalue $\alpha_{k},\left\{W^{k}: k=1,2, \cdots\right\}$ are mutually independent one dimensional Brownian motions and

$$
\sum_{k=1}^{\infty} \frac{\lambda_{k}}{\alpha_{k}}<\infty
$$


Following ([D-Z.2], pp. 260-265), we will transform the mild solution of the stochastic Burgers equation (1.4.39) to that of the random Burgers equation (1.4.42) below. Let $T_{t}: L^{2}([0,1]) \rightarrow$ $L^{2}([0,1]), t \geq 0$, be the heat semi-group on $[0,1]$ with Dirichlet boundary conditions. Let

$$
W_{p}(t)(\xi):=\int_{0}^{t} T_{t-s} d W(s)(\xi)
$$

Then $W_{p}(t)$ has an $C[0,1]$-valued version with Hölder continuous paths ([D-Z.2], Theorem 14.2.4). Set

$$
v(t, \xi):=u(t, \xi)-W_{p}(t)(\xi), \quad t>0, \xi \in[0,1] .
$$

Then $v(t, \xi)$ is a mild solution of the following equation

$$
\frac{\partial v}{\partial t}=\nu \Delta v-\frac{1}{2} \frac{\partial}{\partial \xi}\left(v+W_{p}(t)(\xi)\right)^{2},
$$

in the sense of ([D-Z.2], pp. 260-265).

Viewing equation (1.4.42) as a random Burgers equation, it is not hard to see that, for each initial $\psi \in L^{2}([0,1])$, it has a unique global solution $v(\cdot, \psi, \omega) \in C\left(\mathbf{R}^{+}, L^{2}([0,1])\right) \cap L^{2}\left([0,1], H^{1}[0,1]\right)$ for each $\omega \in \Omega$; and for any $a \in \mathbf{R}^{+}$and any bounded set $S \subset L^{2}([0,1])$, the following holds

$$
\sup _{\substack{t \in[0, a] \\ \psi \in S}}\|v(t, \psi, \omega)\|_{L^{2}([0,1])}<\infty
$$

for all $\omega \in \Omega$ (cf. [Ta], Chapter 15, Proposition 1.3; [D-Z.2], pp. 260-265).

A continuous semi-flow for a stochastic Burgers equation with skew-symmetric noise was obtained in [B-C-F]. However, this is not sufficient for our purposes, since we seek to construct random families of differentiable stable/unstable manifolds near hyperbolic stationary solutions of (1.4.39). In the following theorem, we establish the existence of a perfect $C^{1}$ compacting cocycle for (1.4.39). In Part 2 of this paper, this fact will enable us to use multiplicative ergodic theory techniques in order to prove a local stable/unstable manifold theorem near stationary solutions of the stochastic Burgers equation (1.4.39).

\section{Theorem 1.4.3.}

Consider the stochastic Burgers equation (1.4.39) with $L^{2}[0,1]$-valued Brownian (1.4.40). Then equation (1.4.39) has a mild solution with a $\left(\mathcal{B}\left(\mathbf{R}^{+}\right) \otimes \mathcal{B}\left(L^{2}([0,1])\right) \otimes \mathcal{F}, \mathcal{B}\left(L^{2}([0,1])\right)\right)$-measurable version $u: \mathbf{R}^{+} \times L^{2}([0,1]) \times \Omega \rightarrow L^{2}([0,1])$ having the following properties:

(i) For each $\psi \in L^{2}([0,1]), u(\cdot, \psi, \cdot): \mathbf{R}^{+} \times \Omega \rightarrow L^{2}([0,1])$ is $\left(\mathcal{F}_{t}\right)_{t \geq 0}$-adapted.

(ii) $(u, \theta)$ is a $C^{1}$ perfect cocycle on $L^{2}([0,1])$ (in the sense of Definition 1.1.2).

(iii) For each $(t, \omega) \in(0, \infty) \times \Omega$, the map $L^{2}([0,1]) \ni \psi \mapsto u(t, \psi, \omega) \in L^{2}([0,1])$ takes bounded sets into relatively compact sets.

(iv) For each $(t, \psi, \omega) \in(0, \infty) \times L^{2}([0,1]) \times \Omega$, the Fréchet derivative $D u(t, \psi, \omega) \in L\left(L^{2}([0,1])\right)$ is compact. Furthermore, the map

$$
[0, \infty) \times L^{2}([0,1]) \times \Omega \ni(t, \psi, \omega) \mapsto D u(t, \psi, \omega) \in L\left(L^{2}([0,1])\right)
$$


is strongly measurable.

(v) For any positive reals $a, \rho$,

$$
E \log ^{+} \sup _{\substack{0 \leq t \leq a \\\|\psi\|_{L^{2}([0,1])} \leq \rho}}\left\{\|u(t, \psi, \cdot)\|_{L^{2}([0,1])}+\|D u(t, \psi, \cdot)\|_{L\left(L^{2}([0,1])\right)}\right\}<\infty .
$$

Proof.

For simplicity of notation, we will assume throughout this proof that $\nu=\frac{1}{2}$.

Assertion (i) follows easily from the global existence of solutions to (1.4.42).

To prove (ii), consider Burgers equation (1.4.42). Denote by $p(t, \xi, y)$ the heat kernel for the Laplacian $\nu \Delta$ on $[0,1]$ with Dirichlet boundary conditions. Recall that there are positive constants $c_{1}, c_{2}$ such that

$$
\left|\frac{\partial p(t, \xi, y)}{\partial y}\right| \leq \frac{c_{1}}{t} \mathrm{e}^{-\frac{(\xi-y)^{2}}{2 c_{2} t}}
$$

for all $t>0, \xi, y \in[0,1]$ (c.f. [L-S-U], p. 413). Then pick a positive constant $c_{3}$ such that

$$
\int_{0}^{1} \frac{c_{3}}{\sqrt{t}} \mathrm{e}^{-\frac{y^{2}}{2 c_{2} t}} d y \leq 1
$$

for all $t>0$.

Using (1.4.42), variation of parameters, and integration by parts, we get

$$
\begin{aligned}
v(t, \psi)(\xi)= & T_{t} \psi(\xi)-\frac{1}{2} \int_{0}^{t} T_{t-s} \nabla v^{2}(s, \psi)(\xi) d s \\
& +\int_{0}^{t} T_{t-s}\left(-\nabla\left(W_{p}(s) v(s, \psi)\right)-W_{p}(s) \nabla W_{p}(s)\right)(\xi) d s \\
= & \int_{0}^{1} p(t, \xi, y) \psi(y) d y-\frac{1}{2} \int_{0}^{t} \int_{0}^{1} p(t-s, \xi, y) \nabla v^{2}(s, \psi)(y) d y d s \\
& +\int_{0}^{t} \int_{0}^{1} p(t-s, \xi, y)\left(-\nabla\left(W_{p}(s) v(s, \psi)\right)-W_{p}(s) \nabla W_{p}(s)\right)(y) d y d s \\
= & \int_{0}^{1} p(t, \xi, y) \psi(y) d y+\frac{1}{2} \int_{0}^{t} \int_{0}^{1} \nabla p(t-s, \xi, y) v^{2}(s, \psi)(y) d y d s \\
& +\int_{0}^{t} \int_{0}^{1} \nabla p(t-s, \xi, y)\left(W_{p}(s) v(s, \psi)+\frac{1}{2} W_{p}(s)^{2}\right)(y) d y d s
\end{aligned}
$$

for all $t \geq 0$. Thus

$$
\begin{aligned}
v(t, \psi+ & h g)(\xi)-v(t, \psi)(\xi) \\
= & h \int_{0}^{1} p(t, \xi, y) g(y) d y+\frac{1}{2} \int_{0}^{t} \int_{0}^{1} \nabla p(t-s, \xi, y)\left(v^{2}(s, \psi+h g)(y)-v^{2}(s, \psi)(y)\right) d y d s \\
& +\int_{0}^{t} \int_{0}^{1} \nabla p(t-s, \xi, y)\left(W_{p}(s)(v(s, \psi+h g)-v(s, \psi))(y) d y d s, \quad t>0 .\right.
\end{aligned}
$$


Squaring both sides of the above equality and integrating with respect $\xi \in[0,1]$, we obtain

$$
\begin{aligned}
& \|v(t, \psi+h g)-v(t, \psi)\|_{L^{2}([0,1])}^{2} \\
& \leq 3 h^{2}\|g\|_{L^{2}([0,1])}^{2}+\frac{3}{4} \int_{0}^{1}\left(\int_{0}^{t} \int_{0}^{1} \nabla p(t-s, \xi, y)\left(v^{2}(s, \psi+h g)(y)-v^{2}(s, \psi)(y)\right) d y d s\right)^{2} d \xi \\
& \quad+3 \int_{0}^{1}\left(\int_{0}^{t} \int_{0}^{1} \nabla p(t-s, \xi, y) W_{p}(s)(y)(v(s, \psi+h g)(y)-v(s, \psi)(y)) d y d s\right)^{2} d \xi \\
& \leq 3 h^{2}\|g\|_{L^{2}([0,1])}^{2} \\
& \quad+\frac{3}{4} \int_{0}^{1}\left(\int_{0}^{t} \frac{1}{\sqrt{t-s}} \int_{0}^{1} \frac{c_{1}}{\sqrt{t-s}} \mathrm{e}^{-\frac{(\xi-y)^{2}}{2 c_{2}(t-s)}}\left(v^{2}(s, \psi+h g)(y)-v^{2}(s, \psi)(y)\right) d y d s\right)^{2} d \xi \\
& \quad+3 \int_{0}^{1}\left(\int_{0}^{t} \frac{1}{\sqrt{t-s}} \int_{0}^{1} \frac{c_{1}}{\sqrt{t-s}} \mathrm{e}^{-\frac{(\xi-y)^{2}}{2 c_{2}(t-s)}} W_{p}(s)(y) \times\right. \\
& \times(v(s, \psi+h g)(y)-v(s, \psi)(y)) d y d s)^{2} d \xi,
\end{aligned}
$$

for all $t>0$. Now use Cauchy-Schwartz inequality, the heat kernel estimate (1.4.44) and Fubini's theorem to obtain

$$
\begin{aligned}
\| v(t, \psi+h g)- & v(t, \psi)\left\|_{L^{2}([0,1])}^{2} \leq 3 h^{2}\right\| g \|_{L^{2}([0,1])}^{2}+\frac{3}{4} \int_{0}^{1} \int_{0}^{t} \frac{1}{(t-s)^{\frac{3}{4}}} d s \int_{0}^{t} \frac{1}{(t-s)^{\frac{1}{4}}} \\
& \left(\int_{0}^{1} \frac{c_{1}}{\sqrt{t-s}} \mathrm{e}^{-\frac{(\xi-y)^{2}}{2 c_{2}(t-s)}}\left(v^{2}(s, \psi+h g)(y)-v^{2}(s, \psi)(y)\right) d y\right)^{2} d s d \xi \\
& +3 \int_{0}^{1} \int_{0}^{t} \frac{1}{(t-s)^{\frac{3}{4}}} d s \int_{0}^{t} \frac{1}{(t-s)^{\frac{1}{4}}} \\
& \left(\int_{0}^{1} \frac{c_{1}}{\sqrt{t-s}} \mathrm{e}^{-\frac{(\xi-y)^{2}}{2 c_{2}(t-s)}} W_{p}(s)(v(s, \psi+h g)-v(s, \psi))(y) d y\right)^{2} d s d \xi \\
\leq & 3 h^{2}\|g\|_{L^{2}([0,1])}^{2}+C t^{\frac{1}{4}} \int_{0}^{t} \frac{1}{(t-s)^{\frac{3}{4}}}\|v(s, \psi+h g)-v(s, \psi)\|_{L^{2}([0,1])}^{2} d s \\
& +C t^{\frac{1}{4}} \int_{0}^{t} \frac{1}{(t-s)^{\frac{1}{4}}}\|v(s, \psi+h g)-v(s, \psi)\|_{L^{2}([0,1])} d s, \quad t>0
\end{aligned}
$$

for all $t>0$ and some positive (random) constant $C$. Iterating the above computation, we get

$$
\begin{aligned}
&\|v(t, \psi+h g)-v(t, \psi)\|_{L^{2}([0,1])}^{2} \\
& \leq 3 h^{2}\|g\|_{L^{2}([0,1])}^{2}+C t^{\frac{1}{4}} \int_{0}^{t}\left(\frac{1}{(t-s)^{\frac{3}{4}}}+\frac{1}{(t-s)^{\frac{1}{4}}}\right) 3 h^{2}\|g\|_{L^{2}([0,1])}^{2} d s \\
&+C^{2} t^{\frac{1}{4}} \int_{0}^{t} \int_{0}^{s} s^{\frac{1}{4}}\left(\frac{1}{(t-s)^{\frac{3}{4}}}+\frac{1}{(t-s)^{\frac{1}{4}}}\right)\left(\frac{1}{(s-r)^{\frac{3}{4}}}+\frac{1}{(s-r)^{\frac{1}{4}}}\right) \times \\
& \times\|v(r, \psi+h g)-v(r, \psi)\|_{L^{2}([0,1])}^{2} d r d s, \quad t>0 .
\end{aligned}
$$


Consider now the elementary estimate

$$
\int_{r}^{t} \frac{s^{\alpha}}{(t-s)^{\beta}(s-r)^{\gamma}} d s=\int_{0}^{t-r} \frac{(s+r)^{\alpha}}{(t-r-s)^{\beta} s^{\gamma}} d s \leq \frac{C_{1}}{(t-r)^{\beta+\gamma-1}}, \quad t \geq r>0
$$

which holds for any $\alpha \geq 0,0 \leq \beta<1,0 \leq \gamma<1$, and where $C_{1}>0$ is a positive (deterministic) constant. Using the above estimate together with Fubini's theorem, gives

$$
\begin{aligned}
& \|v(t, \psi+h g)-v(t, \psi)\|_{L^{2}([0,1])}^{2} \\
& \leq 3 h^{2}\|g\|_{L^{2}([0,1])}^{2}+3 C\left(4 t^{\frac{1}{2}}+\frac{4}{3} t\right) h^{2}\|g\|_{L^{2}([0,1])}^{2} \\
& +C^{2} t^{\frac{1}{4}} \int_{0}^{t}\|v(r, \psi+h g)-v(r, \psi)\|_{L^{2}([0,1])}^{2} \times \\
& \quad \times \int_{r}^{t} s^{\frac{1}{4}}\left(\frac{1}{(t-s)^{\frac{3}{4}}}+\frac{1}{(t-s)^{\frac{1}{4}}}\right)\left(\frac{1}{(s-r)^{\frac{3}{4}}}+\frac{1}{(s-r)^{\frac{1}{4}}}\right) d s d r \\
& \leq 3 h^{2}\|g\|_{L^{2}([0,1])}^{2}+3 C\left(4 t^{\frac{1}{2}}+\frac{4}{3} t\right) h^{2}\|g\|_{L^{2}([0,1])}^{2} \\
& +C_{2}\left(t+t^{\frac{1}{4}}\right) \int_{0}^{t} \frac{1}{(t-r)^{1 / 2}}\|v(r, \psi+h g)-v(r, \psi)\|_{L^{2}([0,1])}^{2} d r
\end{aligned}
$$

for all $t \in(0, a], a \in \mathbf{R}^{+}$. Iterating the above process once more and applying Gronwall's lemma, we obtain

$$
\sup _{\substack{0 \leq t \leq a, g \in L^{2}([0,1]) \\\|g\|_{L^{2}} \leq 1}}\|v(t, \psi+h g)-v(t, \psi)\|_{L^{2}([0,1])}^{2} \leq M h^{2},
$$

for any $a \in \mathbf{R}^{+}$, where $M$ is a positive random constant depending on $a$.

For fixed $\psi, g \in L^{2}([0,1])$, define $G:=G(t, \psi)(g)(\xi), t>0, \xi \in[0,1]$, to be the weak solution of the "linearized" Burgers equation

$$
\frac{\partial G}{\partial t}+\frac{\partial(v(t, \psi) G)}{\partial \xi}=\frac{1}{2} \Delta G-\frac{\partial\left(W_{p} G\right)}{\partial \xi}, \quad G(0, \psi)(g)=g \in L^{2}([0,1]) .
$$

Set

$$
\mu_{t}(\psi, g):=v(t, \psi+h g)-v(t, \psi)-h G(t, \psi)(g), \quad|h|<1, t \geq 0 .
$$

Then it is easy to see that

$$
\begin{aligned}
\mu_{t}(\psi, g)(\xi)= & -\int_{0}^{t} \int_{0}^{1} p(t-s, \xi, y)\left(\frac{1}{2} \nabla(v(s, \psi+h g)(y)-v(s, \psi)(y))^{2}\right. \\
\left.\quad+\nabla\left(v(s, \psi)(y) \mu_{s}(\psi, g)(y)\right)+\nabla\left(W_{p}(s)(y) \mu_{s}(\psi, g)(y)\right)\right) d y d s & \int_{0}^{t} \int_{0}^{1} \nabla p(t-s, \xi, y)\left(\frac{1}{2}(v(s, \psi+h g)(y)-v(s, \psi)(y))^{2}\right. \\
& \left.+v(s, \psi)(y) \mu_{s}(\psi, g)(y)+W_{p}(s)(y) \mu_{s}(\psi, g)(y)\right) d y d s
\end{aligned}
$$


for all $t>0$. So using the Cauchy-Schwartz inequality and (1.4.46), we obtain

$$
\begin{aligned}
\| \mu_{t}( & \psi, g) \|_{L^{2}([0,1])}^{2} \\
\leq & \frac{3}{4} \int_{0}^{1}\left(\int_{0}^{t} \int_{0}^{1} \nabla p(t-s, \xi, y)(v(s, \psi+h g)(y)-v(s, \psi)(y))^{2} d y d s\right)^{2} d \xi \\
& +3 \int_{0}^{1}\left(\int_{0}^{t} \int_{0}^{1} \nabla p(t-s, \xi, y)\left(v(s, \psi)(y) \mu_{s}(\psi, g)(y) d y d s\right)^{2} d \xi\right. \\
& +3 \int_{0}^{1}\left(\int_{0}^{t} \int_{0}^{1} \nabla p(t-s, \xi, y)\left(W_{p}(s)(y) \mu_{s}(\psi, g)(y)\right) d y d s\right)^{2} d \xi \\
\leq & \frac{3}{4} \int_{0}^{1} \int_{0}^{t} \frac{1}{(t-s)^{\frac{3}{4}}} d s \int_{0}^{t} \frac{1}{(t-s)^{\frac{1}{4}}}\left(\int_{0}^{1} \frac{c_{1}}{\sqrt{t-s}} e^{-\frac{(y-\xi)^{2}}{c_{2}(t-s)}}\right. \\
& +3 \int_{0}^{1} \int_{0}^{t} \frac{1}{(t-s)^{\frac{3}{4}}} d s \int_{0}^{t} \frac{1}{(t-s)^{\frac{1}{4}}}\left(\int_{0}^{1} \frac{c_{1}}{\sqrt{t-s}} e^{-\frac{(y-\xi)^{2}}{c_{2}(t-s)}}\right. \\
& \left.+3 \int_{0}^{1} \int_{0}^{t} \frac{1}{(t-s)^{\frac{3}{4}}} d s \int_{0}^{t} \frac{1}{(t-s)^{\frac{1}{4}}}\left(\int_{0}^{1} \frac{c_{1}}{\sqrt{t-s}} e^{-\frac{(y-\xi)^{2}}{c_{2}(t-s)}} d s d \xi\right)(y) \mu_{s}(\psi, g)(y) d y\right)^{2} d s d \xi \\
& \left.\left(W_{p}(s)(y) \mu_{s}(\psi, g)(y)\right) d y\right)^{2} d s d \xi .
\end{aligned}
$$

for all $t>0$. Thus

$$
\begin{aligned}
\left\|\mu_{t}(\psi, g)\right\|_{L^{2}([0,1]) \leq}^{2} \leq & 3 c_{1} t^{\frac{1}{4}} \int_{0}^{t} \frac{1}{(t-s)^{\frac{3}{4}}}\left(\int_{0}^{1}(v(s, \psi+h g)(y)-v(s, \psi)(y))^{2} d y\right)^{2} d s \\
& +12 c_{1} t^{\frac{1}{4}} \int_{0}^{t} \frac{1}{(t-s)^{\frac{3}{4}}} \int_{0}^{1} v^{2}(s, \psi)(y) d y \int_{0}^{1} \mu_{s}^{2}(\psi, g)(y) d y d s \\
& +12 t^{\frac{1}{4}}\|b\|_{\infty}^{2} \int_{0}^{t} \frac{1}{(t-s)^{\frac{1}{4}}} \int_{0}^{1} \mu_{s}^{2}(\psi, g)(y) d y d s \\
\leq & C h^{4}+C \int_{0}^{t}\left(\frac{1}{(t-s)^{\frac{3}{4}}}+\frac{1}{(t-s)^{\frac{1}{4}}}\right) \| \mu_{s}\left(\psi, g \|_{L^{2}([0,1])}^{2} d s, \quad 0 \leq t \leq a,\right.
\end{aligned}
$$

where $C=C(\omega, a)$ is a positive random constant. Using the previous iteration argument followed by Gronwall's lemma, we obtain the following estimate

$$
\sup _{\substack{g \in L^{2}([0,1]),\|g \mid\|_{L^{2}} \leq 1 \\ 0 \leq t \leq a}}\left\|\mu_{t}(\psi, g)\right\|_{L^{2}([0,1])}^{2} \leq M_{1} h^{4}, \quad|h|<1
$$

for some positive random constant $M_{1}=M_{1}(\omega, a)$. This implies that $\frac{\mu_{t}(\psi, g)}{h}$ converges to 0 as $h \rightarrow 0$ in $L^{2}([0,1])$, uniformly in $(t, g) \in[0, a] \times\left\{g \in L^{2}([0,1]):\|g\|_{L^{2}} \leq 1\right\}$. Therefore, 
$\frac{v(t, \psi+h g)-v(t, \psi)}{h} \rightarrow G_{t}(\psi, g)$ as $h \rightarrow 0$, uniformly for $g \in\left\{g ;\|g\|_{L^{2}([0,1])} \leq 1\right\}$. Hence, $v$ is Fréchet differentiable at $\psi \in L^{2}([0,1])$, with Fréchet derivative $D v(t, \psi): L^{2}([0,1]) \rightarrow L^{2}([0,1])$ satisfying the $L\left(L^{2}([0,1])\right)$-valued linear equation

$$
\begin{aligned}
D v(t, \psi)= & T_{t}-\int_{0}^{t} T_{t-s}\left(\frac{\partial v(s, \psi)}{\partial \xi} D v(s, \psi)+v(s, \psi) \frac{\partial D v(s, \psi)}{\partial \xi}\right) d s \\
& -\int_{0}^{t} T_{t-s}\left(D v(s, \psi) \frac{\partial W_{p}(s)}{\partial \xi}+W_{p}(s) \frac{\partial D v(s, \psi)}{\partial \xi}\right) d s
\end{aligned}
$$

for $(t, \psi) \in \mathbf{R}^{+} \times L^{2}([0,1])$.

In order to complete the proof of assertion (ii) of the theorem, it remains to prove that $(u, \theta)$ is a perfect cocycle in $L^{2}([0,1])$. It is easy to see from (1.4.39) that

$$
u(t, \psi)(\omega)=T_{t} \psi-\int_{0}^{t} T_{t-s} u(s, \psi, \omega) \nabla u(s, \psi, \omega) d s+\left[\int_{0}^{t} T_{t-s} d W(s)\right](\omega)
$$

for $t>0, \omega \in \Omega, \psi \in L^{2}([0,1])$. We need to prove that

$$
u\left(t, u\left(t_{1}, \psi, \omega\right), \theta\left(t_{1}, \omega\right)\right)=u\left(t+t_{1}, \psi, \omega\right)
$$

for $t, t_{1} \geq 0, \omega \in \Omega, \psi \in L^{2}([0,1])$. To see this, fix $t_{1} \geq 0, \omega \in \Omega, \psi \in L^{2}([0,1])$, and denote

$$
Y(t):=u\left(t, u\left(t_{1}, \psi, \omega\right), \theta\left(t_{1}, \omega\right)\right), \quad Z(t):=u\left(t+t_{1}, \psi, \omega\right), \quad t>0 .
$$

Then

$$
\begin{aligned}
Y(t)= & T_{t} u\left(t_{1}, \psi, \omega\right)-\int_{0}^{t} T_{t-s} u\left(s, u\left(t_{1}, \psi, \omega\right), \theta\left(t_{1}, \omega\right)\right) \frac{\partial u\left(s, u\left(t_{1}, \psi, \omega\right), \theta\left(t_{1}, \omega\right)\right)}{\partial y} d s \\
& +\left[\int_{t_{1}}^{t+t_{1}} T_{t+t_{1}-s} d W(s)\right](\omega) \\
= & T_{t+t_{1}} \psi-\int_{0}^{t_{1}} T_{t+t_{1}-s} u(s, \psi, \omega) \frac{\partial u(s, \psi, \omega)}{\partial y} d s \\
& -\int_{t_{1}}^{t+t_{1}} T_{t+t_{1}-s} Y\left(s-t_{1}\right) \frac{\partial Y\left(s-t_{1}\right)}{\partial y} d s+\left[\int_{0}^{t+t_{1}} T_{t+t_{1}-s} d W(s)\right](\omega), \quad t>0 .
\end{aligned}
$$

Also,

$$
\begin{aligned}
Z(t)= & T_{t+t_{1}} \psi-\int_{0}^{t_{1}} T_{t+t_{1}-s} u(s, \psi, \omega) \frac{\partial u(s, \psi, \omega)}{\partial y} d s \\
& -\int_{t_{1}}^{t+t_{1}} T_{t+t_{1}-s} Z\left(s-t_{1}\right) \frac{\partial Z\left(s-t_{1}\right)}{\partial y} d s+\left[\int_{0}^{t+t_{1}} T_{t+t_{1}-s} d W(s)\right](\omega), \quad t>0 .
\end{aligned}
$$


Therefore,

$$
\begin{aligned}
Y(t)(\xi)-Z(t)(\xi) & =-\frac{1}{2} \int_{t_{1}}^{t+t_{1}} T_{t+t_{1}-s}\left(\frac{\partial Y^{2}\left(s-t_{1}\right)}{\partial y}-\frac{\partial Z^{2}\left(s-t_{1}\right)}{\partial y}\right)(\xi) d s \\
& =\frac{1}{2} \int_{t_{1}}^{t+t_{1}} d s \int_{0}^{1} \frac{\partial p\left(t+t_{1}-s, \xi, y\right)}{\partial y}\left(Y^{2}\left(s-t_{1}\right)(y)-Z^{2}\left(s-t_{1}\right)(y)\right) d y \\
& =\frac{1}{2} \int_{0}^{t} d s \int_{0}^{1} \frac{\partial p(t-s, \xi, y)}{\partial y}\left(Y^{2}(s)(y)-Z^{2}(s)(y)\right) d y,
\end{aligned}
$$

for $t>0, \xi \in \mathcal{D}$. Using Cauchy-Schwartz inequality and (1.4.43), we have

$$
\begin{aligned}
& \|Y(t)-Z(t)\|_{L^{2}([0,1])}^{2}=\int_{0}^{1}(Y(t)(\xi)-Z(t)(\xi))^{2} d \xi \\
\leq & C \int_{0}^{1} d \xi\left(\frac{1}{2} \int_{0}^{t} d s \int_{0}^{1} \frac{1}{\sqrt{t-s}} \frac{c_{1}}{\sqrt{t-s}} e^{-\frac{(\xi-y)^{2}}{2 c_{2}(t-s)}}\left|Y^{2}(s)(y)-Z^{2}(s)(y)\right| d y\right)^{2} \\
\leq & C \int_{0}^{1} \int_{0}^{t} \frac{1}{(t-s)^{\frac{3}{4}}} d s \int_{0}^{t} \frac{1}{(t-s)^{\frac{1}{4}}}\left(\int_{0}^{1} \frac{c_{1}}{\sqrt{t-s}} e^{-\frac{(\xi-y)^{2}}{2 c_{2}(t-s)}}\left|Y^{2}(s)(y)-Z^{2}(s)(y)\right| d y\right)^{2} d s d \xi \\
\leq & C \int_{0}^{1} \int_{0}^{t} \frac{1}{(t-s)^{\frac{1}{4}}} \int_{0}^{1} \frac{c_{1}}{\sqrt{t-s}} e^{-\frac{(\xi-y)^{2}}{2 c_{2}(t-s)}}(Y(s)(y)-Z(s)(y))^{2} d y \\
\leq & \sup _{0 \leq s \leq T}\left[\|Y(s)+Z(s)\|_{L^{2}([0,1])}^{2}\right] \int_{0}^{t} \frac{1}{(t-s)^{\frac{3}{4}}}\|Y(s)-Z(s)\|_{L^{2}([0,1])}^{2} d s \\
\leq & M(\omega) \int_{0}^{t} \frac{1}{(t-s)^{\frac{3}{4}}}\|Y(s)-Z(s)\|_{L^{2}([0,1])}^{2} d s,
\end{aligned}
$$

for all $t \in(0, a]$, where $C$ is a generic constant that may change from line to line. Note that in the above computation, we have also used the fact that

$$
\sup _{0 \leq s \leq a}\left[\|Y(s)+Z(s)\|_{L^{2}([0,1])}^{2}\right]<\infty, \quad a \in \mathbf{R}^{+} .
$$

As in the proof of (1.4.45) it follows from (1.4.49) and Gronwall's lemma that $Y(t)=Z(t)$ for all $t \geq 0$. This completes the proof of assertion (ii) of the theorem.

To prove assertion (iii), it is easy to see from the proof of (1.4.45) that

$$
\begin{aligned}
& \left\|v\left(t, \psi_{m}, \omega\right)-v\left(t, \psi_{n}, \omega\right)\right\|_{L^{2}([0,1])}^{2} \\
& \quad \leq 3\left\|T_{t} \psi_{m}-T_{t} \psi_{n}\right\|_{L^{2}([0,1])}^{2}+C t \int_{0}^{t}\left(\frac{1}{\sqrt{t-s}}+1\right)\left\|v\left(s, \psi_{m}, \omega\right)-v\left(s, \psi_{n}, \omega\right)\right\|_{L^{2}([0,1])}^{2} d s \\
& \quad+C t^{\frac{1}{4}} \int_{0}^{t}\left(\frac{1}{(t-s)^{\frac{3}{4}}}+\frac{1}{(t-s)^{\frac{1}{4}}}\right)\left\|v\left(s, \psi_{m}, \omega\right)-v\left(s, \psi_{n}, \omega\right)\right\|_{L^{2}([0,1])}^{2} d s,
\end{aligned}
$$

for $t \in[0, a]$ where $C$ is a positive random constant. Now using (1.4.50) and the same argument as in the proof of compactness of $D v(t, \psi, \omega)$ in Theorem 1.4.1, one can show $v(t, \cdot, \omega): L^{2}([0,1]) \rightarrow$ $L^{2}([0,1]), t>0$, takes bounded sets into relatively compact sets. The only difference is that we have to iterate (1.4.50) once before we can use Gronwall's lemma. Details of the proof are omitted. 


\section{Part 2: Existence of Stable and Unstable Manifolds}

\subsection{Hyperbolicity of a stationary trajectory.}

In Part 1, we established the existence of perfect differentiable cocycles generated by mild solutions of a large class of semilinear stochastic evolution equations (see's) and stochastic partial differential equations (spde's). In this part, we continue the analysis in Part 1. More specifically, we highlight the concept of a stationary point for the see or spde as an invariant random vector under the cocycle. Our main objective is to characterize the pathwise local structure of solutions of semilinear see's and spde's near stationary solutions. We define the concept of hyperbolicity for a stationary solution of an see. Hyperbolicity is characterized by the non-vanishing of the Lyapunov spectrum of the linearized cocycle. The hyperbolic structure of the stochastic semiflow leads to local stable manifold theorems (Theorems 2.4.1-2.4.4) for semilinear see's and spde's. For a hyperbolic stationary solution of the see, this gives smooth stable and unstable manifolds in a neighborhood of the stationary solution. The stable and unstable manifolds are stationary, live in a stationary tubular neighborhood of the stationary solution and are asymptotically invariant under the stochastic semiflow of the see/spde. Furthermore, the local stable and unstable manifolds intersect transversally at the stationary point, and the unstable manifolds have fixed finite dimension. Due to their forward asymptotic dependence on the future of the stochastic semiflow, the stable and unstable manifolds are in general anticipating in nature. In particular, the tangent spaces to the stable and unstable manifolds (at the stationary point) are constructed using the (anticipating) eigenspaces of the Oseledec-Ruelle operator for the hyperbolic linearized cocycle.

The proof of the stable manifold theorem (Theorem 2.2.1) uses infinite-dimensional multiplicative ergodic theory techniques ([Ru.1], [Ru.2]) together with interpolation and perfection arguments ([Mo.1], [M-S.4]). In particular, we will assume that the reader is familiar with the results and the techniques in Ruelle's articles [Ru.1] and [Ru.2].

We recall below the definition of a cocycle in Hilbert space.

Let $(\Omega, \mathcal{F}, P)$ be a complete probability space. Suppose $\theta: \mathbf{R} \times \Omega \rightarrow \Omega$ is a group of $P$-preserving ergodic transformations on $(\Omega, \mathcal{F}, P)$. Denote by $\overline{\mathcal{F}}$ the $P$-completion of $\mathcal{F}$.

Let $H$ be a real separable Hilbert space with norm $|\cdot|$ and Borel $\sigma$-algebra $\mathcal{B}(H)$.

Take $k$ to be any non-negative integer and $\epsilon \in(0,1]$. Recall that a $C^{k, \epsilon}$ perfect cocycle $(U, \theta)$ on $H$ is a $\left(\mathcal{B}\left(\mathbf{R}^{+}\right) \otimes \mathcal{B}(H) \otimes \mathcal{F}, \mathcal{B}(H)\right)$ - measurable random field $U: \mathbf{R}^{+} \times H \times \Omega \rightarrow H$ with the following properties:

(i) For each $\omega \in \Omega$, the map $\mathbf{R}^{+} \times H \ni(t, x) \mapsto U(t, x, \omega) \in H$ is continuous; for fixed $(t, \omega) \in \mathbf{R}^{+} \times \Omega$, the map $H \ni x \mapsto U(t, x, \omega) \in H$ is $C^{k, \epsilon}\left(D^{k} U(t, x, \omega)\right.$ is $C^{\epsilon}$ in $x$ on bounded subsets of $H$ ).

(ii) $U\left(t_{1}+t_{2}, \cdot, \omega\right)=U\left(t_{2}, \cdot, \theta\left(t_{1}, \omega\right)\right) \circ U\left(t_{1}, \cdot, \omega\right)$ for all $t_{1}, t_{2} \in \mathbf{R}^{+}$, all $\omega \in \Omega$.

(iii) $U(0, x, \omega)=x$ for all $x \in H, \omega \in \Omega$. 
We now introduce the concept of a stationary point for a cocycle $(U, \theta)$. Stationary points play the role of stochastic equilibria for the stochastic dynamical system.

\section{Definition 2.1.1.}

An $\mathcal{F}$-measurable random variable $Y: \Omega \rightarrow H$ is said be a stationary random point for the cocycle $(U, \theta)$ if it satisfies the following identity:

$$
U(t, Y(\omega), \omega)=Y(\theta(t, \omega))
$$

for all $(t, \omega) \in \mathbf{R}^{+} \times \Omega$.

The reader may note that the above definition is an infinite-dimensional analogue of a corresponding concept of invariance that was used by one of the authors in joint work with M. Scheutzow to give a proof of the stable manifold theorem for stochastic ordinary differential equations (Definition 3.1, [M-S.3]). Definition 2.1.1 essentially gives a useful realization of the idea of an invariant measure for a stochastic dynamical system generated by an spde or a see. Such a realization allows us to analyze the local almost sure stability properties of the stochastic semiflow in the neighborhood of the stationary point. The existence (and uniqueness/ergodicity) of a stationary random point for various classes of spde's and see's has been studied by many researchers. In this article, we move beyond the issue of existence of stationary solutions, and apply our stable/unstable manifold theorem to examine further the almost sure asymptotic structure of the stochastic flow generated by several well-known classes of see's and spde's. In particular, we establish the existence of local stable and unstable manifolds near their stationary points.

We would like to remark that, in general, $Y(\theta(t, \omega))$ is not an adapted process because the stationary point $Y$ may depend on the full Brownian path that drives the spde: See Proposition 2.4.1. Thus, one does not expect that $Y(\theta(t, \omega))$ would solve the underlying Itô-type see or spde. However, it has been established in joint work by one of the authors with M. Scheutzow that, for sode's, such a stationary trajectory does indeed satisfy the corresponding Stratonovich version of the sde ([M-S.2], Theorem A.2). In our present context, we conjecture that an analogous result also holds for the see's and spde's treated in this article.

The main objective of this section is to define the concept of hyperbolicity for a stationary point $Y$ of the cocycle $(U, \theta)$.

First, we linearize the $C^{k, \epsilon}$ cocycle $(U, \theta)$ along a stationary random point $Y$. By taking Fréchet derivatives at $Y(\omega)$ on each side of the cocycle identity (ii) above, using the chain rule and the definition of $Y$, we immediately see that $(D U(t, Y(\omega), \omega), \theta(t, \omega))$ is an $L(H)$-valued perfect cocycle. Secondly, we appeal to the following classical result which goes back to Oseledec in the finite-dimensional case $([\mathrm{O}])$, and to D. Ruelle in infinite dimensions ([Ru.2]). 
Theorem 2.1.1. (Oseledec-Ruelle)

Let $T: \mathbf{R}^{+} \times \Omega \rightarrow L(H)$ be strongly measurable, such that $(T, \theta)$ is an $L(H)$-valued cocycle, with each $T(t, \omega)$ compact. Suppose that

$$
E \sup _{0 \leq t \leq 1} \log ^{+}\|T(t, \cdot)\|_{L(H)}+E \sup _{0 \leq t \leq 1} \log ^{+}\|T(1-t, \theta(t, \cdot))\|_{L(H)}<\infty .
$$

Then there is a sure event $\Omega_{0} \in \mathcal{F}$ such that $\theta(t, \cdot)\left(\Omega_{0}\right) \subseteq \Omega_{0}$ for all $t \in \mathbf{R}^{+}$, and for each $\omega \in \Omega_{0}$, the limit

$$
\Lambda(\omega):=\lim _{t \rightarrow \infty}\left[T(t, \omega)^{*} \circ T(t, \omega)\right]^{1 /(2 t)}
$$

exists in the uniform operator norm. Each linear operator $\Lambda(\omega)$ is compact, non-negative and self-adjoint with a discrete spectrum

$$
e^{\lambda_{1}}>e^{\lambda_{2}}>e^{\lambda_{3}}>\cdots
$$

where the $\lambda_{i}$ 's are distinct and non-random. Each eigenvalue $e^{\lambda_{i}}>0$ has a fixed finite non-random multiplicity $m_{i}$ and a corresponding eigen-space $F_{i}(\omega)$, with $m_{i}:=\operatorname{dim} F_{i}(\omega)$. Set $i=\infty$ when $\lambda_{i}=-\infty$. Define

$$
E_{1}(\omega):=H, \quad E_{i}(\omega):=\left[\oplus_{j=1}^{i-1} F_{j}(\omega)\right]^{\perp}, i>1, E_{\infty}:=\operatorname{ker} \Lambda(\omega)
$$

Then

$$
\begin{gathered}
E_{\infty} \subset \cdots \subset \cdots \subset E_{i+1}(\omega) \subset E_{i}(\omega) \cdots \subset E_{2}(\omega) \subset E_{1}(\omega)=H, \\
\lim _{t \rightarrow \infty} \frac{1}{t} \log |T(t, \omega) x|=\left\{\begin{array}{l}
\lambda_{i} \text { if } x \in E_{i}(\omega) \backslash E_{i+1}(\omega), \\
-\infty \text { if } x \in E_{\infty}(\omega),
\end{array}\right.
\end{gathered}
$$

and

$$
T(t, \omega)\left(E_{i}(\omega)\right) \subseteq E_{i}(\theta(t, \omega))
$$

for all $t \geq 0, i \geq 1$.

Fig. 2 illustrates the Oseledec-Ruelle theorem. 


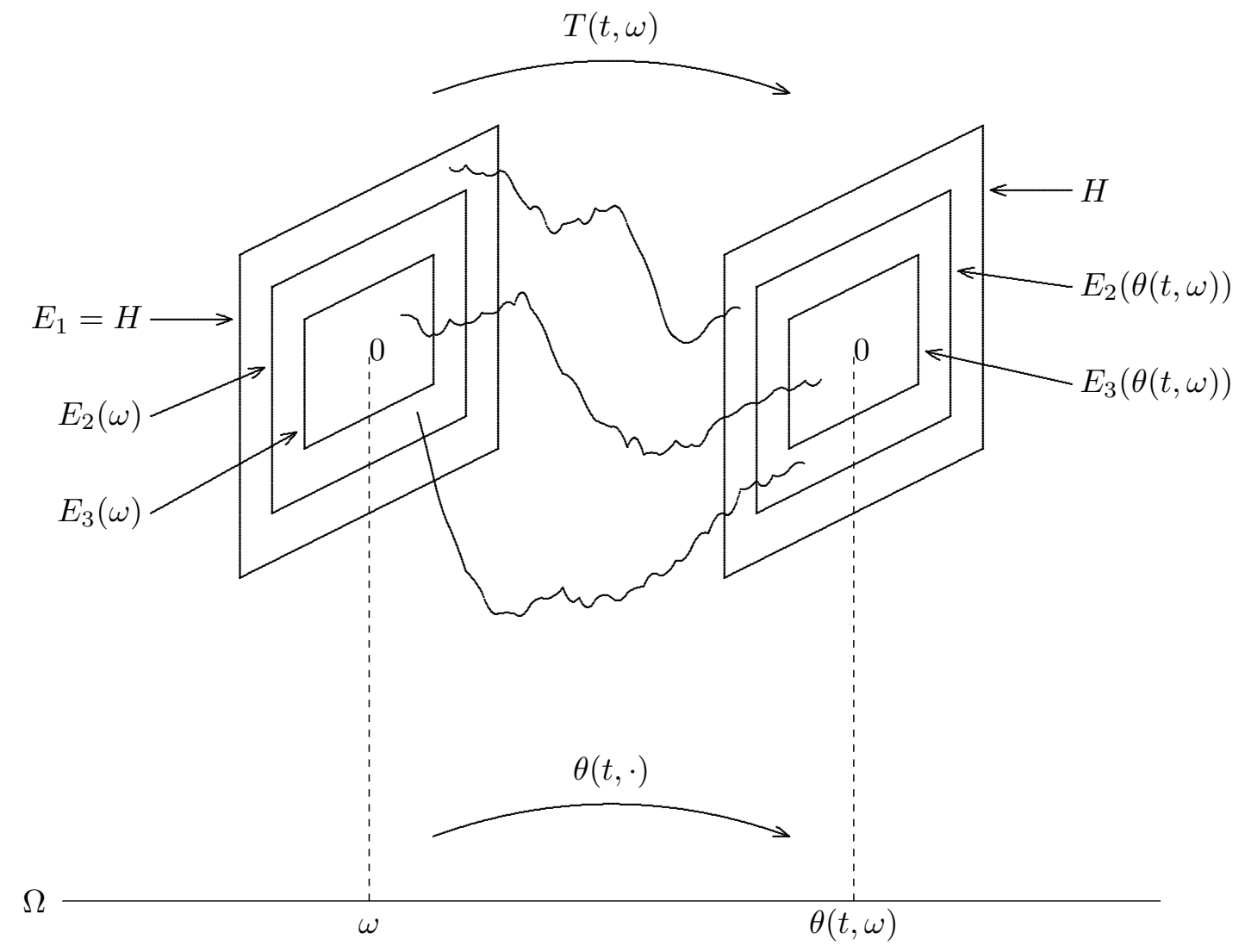

Fig. 2: The Spectral Theorem

Proof of Theorem 2.1.1.

The proof is based on a discrete version of Oseledec's multiplicative ergodic theorem and the perfect ergodic theorem ([Ru.1], I.H.E.S Publications, 1979, pp. 303-304; cf. [O], [Mo.1], Lemma 5. See also Lemma 2.3.1 (ii) of this article). Details of the extension to continuous time are given in [Mo.1] within the context of linear stochastic functional differential equations. The arguments in [Mo.1] extend directly to general linear cocycles in Hilbert space. cf. [F-S].

\section{Definition 2.1.2.}

The sequence $\left\{\cdots<\lambda_{i+1}<\lambda_{i}<\cdots<\lambda_{2}<\lambda_{1}\right\}$ in the Oseledec-Ruelle theorem (Theorem 2.1.1) is called the Lyapunov spectrum of the linear cocycle $(T, \theta)$.

Hyperbolicity of a stationary point $Y: \Omega \rightarrow H$ of the non-linear cocycle $(U, \theta)$ may now be defined in terms of a spectral gap in the Lyapunov spectrum of the linearized cocycle $(D U(t, Y(\omega), \omega), \theta(t, \omega))$. 


\section{Definition 2.1.3.}

Let $(U, \theta)$ be a $C^{k, \epsilon}(k \geq 1, \epsilon \in(0,1])$ perfect cocycle on a separable Hilbert space $H$ such that $U(t, \cdot, \omega): H \rightarrow H$ takes bounded sets into relatively compact sets for each $(t, \omega) \in(0, \infty) \times \Omega$. A stationary point $Y(\omega)$ of the cocycle $(U, \theta)$ is hyperbolic if

(a) For any $a \in(0, \infty)$,

$$
\int_{\Omega} \log ^{+} \sup _{0 \leq t_{1}, t_{2} \leq a}\left\|D U\left(t_{2}, Y\left(\theta\left(t_{1}, \omega\right)\right), \theta\left(t_{1}, \omega\right)\right)\right\|_{L(H)} d P(\omega)<\infty .
$$

(b) The linearized cocycle $(D U(t, Y(\omega), \omega), \theta(t, \omega))$ has a non-vanishing Lyapunov spectrum $\left\{\cdots<\lambda_{i+1}<\lambda_{i}<\cdots<\lambda_{2}<\lambda_{1}\right\}$, viz. $\lambda_{i} \neq 0$ for all $i \geq 1$.

By the Oseledec theorem (Theorem 2.1.1), the integrability condition in Definition 2.1.2 (a) implies the existence of a discrete Lyapunov spectrum for the linearized cocycle $(D U(t, Y(\omega), \omega), \theta(t, \omega))$ in Definition 2.1.2 (b) above.

The following result is a random version of the saddle point property for hyperbolic linear cocycles. A proof is given in ([Mo.1], Theorem 4, Corollary 2; [M-S.1], Theorem 5.3) within the context of stochastic differential systems with memory; but the arguments therein extend immediately to linear cocycles in Hilbert space.

Theorem 2.1.2. (Stable and unstable subspaces)

Let $(T, \theta)$ be a linear cocycle on a Hilbert space $H$. Assume that $T(t, \omega): H \rightarrow H$ is a compact linear operator for each $t>0$ and a.a. $\omega \in \Omega$. Suppose that

$$
E \log ^{+} \sup _{0 \leq t_{1}, t_{2} \leq 1}\left\|T\left(t_{2}, \theta\left(t_{1}, \cdot\right)\right)\right\|_{L(H)}<\infty
$$

and let the cocycle $(T, \theta)$ have a non-vanishing Lyapunov spectrum $\left\{\cdots<\lambda_{i+1}<\lambda_{i}<\cdots<\lambda_{2}<\right.$ $\left.\lambda_{1}\right\}$. Pick $i_{0}>1$ such that $\lambda_{i_{0}}<0<\lambda_{i_{0}-1}$.

Then there is a sure event $\Omega^{*} \in \mathcal{F}$ and stable and unstable subspaces $\left\{S(\omega), U(\omega): \omega \in \Omega^{*}\right\}$, $\mathcal{F}$-measurable (into the Grassmanian), such that for each $\omega \in \Omega^{*}$, the following is true:

(i) $\theta(t, \cdot)\left(\Omega^{*}\right)=\Omega^{*}$ for all $t \in \mathbf{R}$.

(ii) $H=\mathcal{U}(\omega) \oplus \mathcal{S}(\omega)$. The subspace $\mathcal{U}(\omega)$ is finite-dimensional with a fixed non-random dimension, and $\mathcal{S}(\omega)$ is closed with a finite non-random codimension.

(iii) (Invariance)

$$
T(t, \omega)(\mathcal{U}(\omega))=\mathcal{U}(\theta(t, \omega)), T(t, \omega)(\mathcal{S}(\omega)) \subseteq \mathcal{S}(\theta(t, \omega))
$$

for all $t \geq 0$,

(iv) (Exponential dichotomies)

$$
|T(t, \omega)(x)| \geq|x| e^{\delta_{1} t} \quad \text { for all } \quad t \geq \tau_{1}^{*}, x \in \mathcal{U}(\omega)
$$




$$
|T(t, \omega)(x)| \leq|x| e^{-\delta_{2} t} \quad \text { for all } \quad t \geq \tau_{2}^{*}, x \in \mathcal{S}(\omega),
$$

where $\tau_{i}^{*}=\tau_{i}^{*}(x, \omega)>0, i=1,2$, are random times and $\delta_{i}>0, i=1,2$, are fixed.

This theorem is illustrated in Fig. 3.

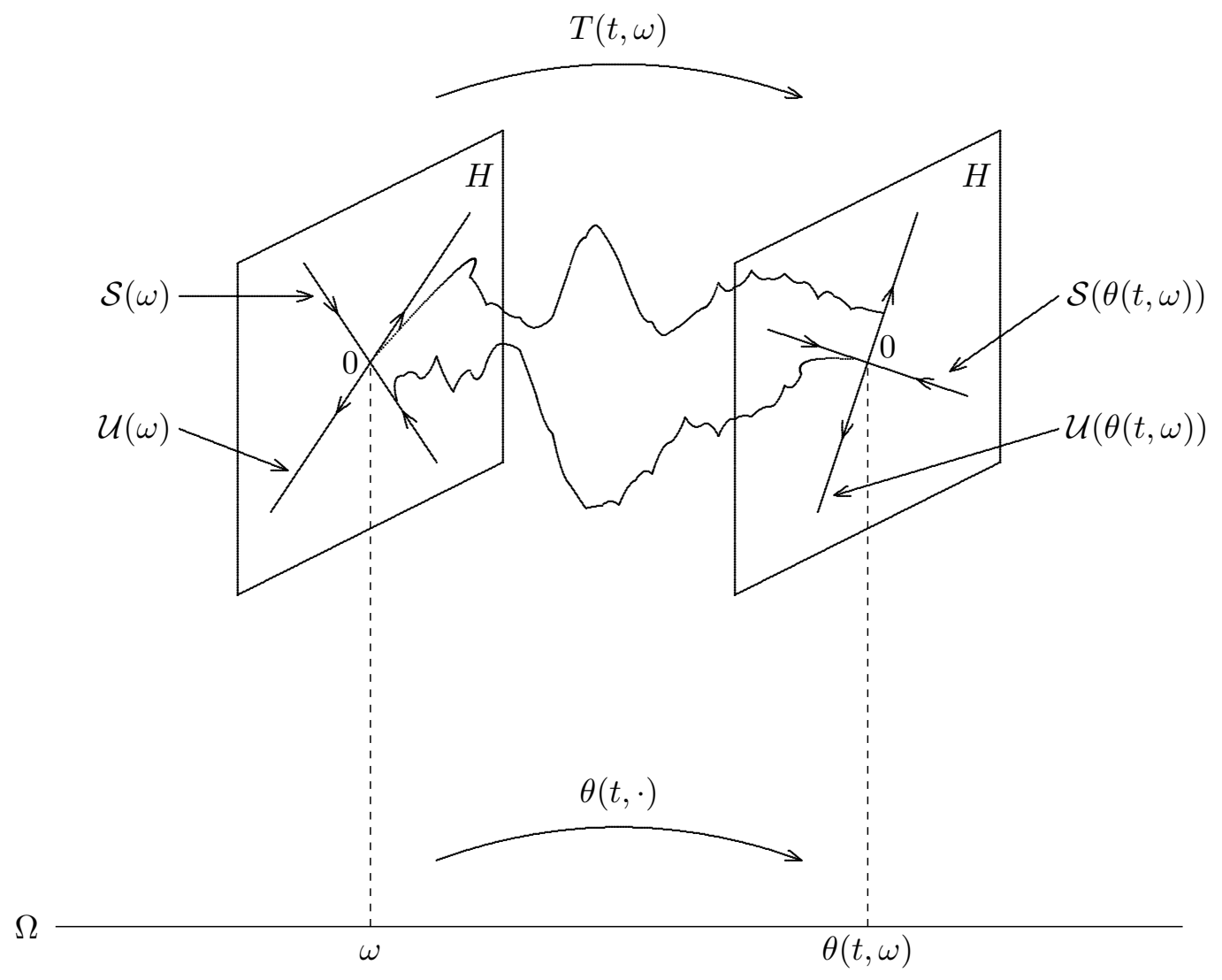

Fig. 3: The stable and unstable subspaces 


\subsection{The non-linear ergodic theorem.}

The main objective of this section is to refine and extend discrete-time results of D. Ruelle to the continuous-time setting in Theorem 2.2.1 below. This setting underlies the dynamics of the semilinear see's and spde's studied in Part 1. As will be apparent later, the extension of Ruelle's results to continuous-time is non-trivial. Indeed, Section 2.3 in its entirety is devoted to the proof of Theorem 2.2.1. The main difficulties in the analysis are outlined after the statement of the theorem.

In the following, denote by $B(x, \rho)$ the open ball in $H$, radius $\rho$ and center $x \in H$, and by $\bar{B}(x, \rho)$ the corresponding closed ball.

Theorem 2.2.1. (The local stable manifold theorem)

Let $(U, \theta)$ be a $C^{k, \epsilon}(k \geq 1, \epsilon \in(0,1])$ perfect cocycle on a separable Hilbert space $H$ such that for each $(t, \omega) \in(0, \infty) \times \Omega, U(t, \cdot, \omega): H \rightarrow H$ takes bounded sets into relatively compact sets. For any $\rho \in(0, \infty)$, denote by $\|\cdot\|_{k, \epsilon}$ the $C^{k, \epsilon}{ }_{-n o r m}$ on the space $C^{k, \epsilon}(\bar{B}(0, \rho), H)$. Let $Y$ be a hyperbolic stationary point of the cocycle $(U, \theta)$ satisfying the following integrability property:

$$
\int_{\Omega} \log ^{+} \sup _{0 \leq t_{1}, t_{2} \leq a}\left\|U\left(t_{2}, Y\left(\theta\left(t_{1}, \omega\right)\right), \theta\left(t_{1}, \omega\right)\right)\right\|_{k, \epsilon} d P(\omega)<\infty
$$

for any fixed $0<\rho, a<\infty$ and $\epsilon \in(0,1]$. Denote by $\left\{\cdots<\lambda_{i+1}<\lambda_{i}<\cdots<\lambda_{2}<\lambda_{1}\right\}$ the Lyapunov spectrum of the linearized cocycle $(D U(t, Y(\omega), \omega), \theta(t, \omega), t \geq 0)$. Define $\lambda_{i_{0}}:=\max \left\{\lambda_{i}\right.$ : $\left.\lambda_{i}<0\right\}$ if at least one $\lambda_{i}<0$. If all finite $\lambda_{i}$ are positive, set $\lambda_{i_{0}}:=-\infty$. (Thus $\lambda_{i_{0}-1}$ is the smallest positive Lyapunov exponent of the linearized cocycle, if at least one $\lambda_{i}>0$; in case all the $\lambda_{i}$ 's are negative, set $\lambda_{i_{0}-1}:=\infty$.)

Fix $\epsilon_{1} \in\left(0,-\lambda_{i_{0}}\right)$ and $\epsilon_{2} \in\left(0, \lambda_{i_{0}-1}\right)$. Then there exist

(i) a sure event $\Omega^{*} \in \mathcal{F}$ with $\theta(t, \cdot)\left(\Omega^{*}\right)=\Omega^{*}$ for all $t \in \mathbf{R}$,

(ii) $\overline{\mathcal{F}}$-measurable random variables $\rho_{i}, \beta_{i}: \Omega^{*} \rightarrow(0,1), \beta_{i}>\rho_{i}>0, i=1,2$, such that for each $\omega \in \Omega^{*}$, the following is true:

There are $C^{k, \epsilon}(\epsilon \in(0,1])$ submanifolds $\tilde{\mathcal{S}}(\omega), \tilde{\mathcal{U}}(\omega)$ of $\bar{B}\left(Y(\omega), \rho_{1}(\omega)\right)$ and $\bar{B}\left(Y(\omega), \rho_{2}(\omega)\right)$ (resp.) with the following properties:

(a) For $\lambda_{i_{0}}>-\infty, \tilde{\mathcal{S}}(\omega)$ is the set of all $x \in \bar{B}\left(Y(\omega), \rho_{1}(\omega)\right)$ such that

$$
|U(n, x, \omega)-Y(\theta(n, \omega))| \leq \beta_{1}(\omega) e^{\left(\lambda_{i_{0}}+\epsilon_{1}\right) n}
$$

for all integers $n \geq 0$. If $\lambda_{i_{0}}=-\infty$, then $\tilde{\mathcal{S}}(\omega)$ is the set of all $x \in \bar{B}\left(Y(\omega), \rho_{1}(\omega)\right)$ such that

$$
|U(n, x, \omega)-Y(\theta(n, \omega))| \leq \beta_{1}(\omega) e^{\lambda n}
$$

for all integers $n \geq 0$ and any $\lambda \in(-\infty, 0)$. Furthermore,

$$
\limsup _{t \rightarrow \infty} \frac{1}{t} \log |U(t, x, \omega)-Y(\theta(t, \omega))| \leq \lambda_{i_{0}}
$$


for all $x \in \tilde{\mathcal{S}}(\omega)$. Each stable subspace $\mathcal{S}(\omega)$ of the linearized cocycle $(D U(t, Y(\cdot), \cdot), \theta(t, \cdot))$ is tangent at $Y(\omega)$ to the submanifold $\tilde{\mathcal{S}}(\omega)$, viz. $T_{Y(\omega)} \tilde{\mathcal{S}}(\omega)=\mathcal{S}(\omega)$. In particular, codim $\tilde{\mathcal{S}}(\omega)=\operatorname{codim} \mathcal{S}(\omega)$, is fixed and finite.

(b) $\limsup _{t \rightarrow \infty} \frac{1}{t} \log \left[\sup \left\{\frac{\left|U\left(t, x_{1}, \omega\right)-U\left(t, x_{2}, \omega\right)\right|}{\left|x_{1}-x_{2}\right|}: x_{1} \neq x_{2}, x_{1}, x_{2} \in \tilde{\mathcal{S}}(\omega)\right\}\right] \leq \lambda_{i_{0}}$.

(c) (Cocycle-invariance of the stable manifolds):

There exists $\tau_{1}(\omega) \geq 0$ such that

$$
U(t, \cdot, \omega)(\tilde{\mathcal{S}}(\omega)) \subseteq \tilde{\mathcal{S}}(\theta(t, \omega))
$$

for all $t \geq \tau_{1}(\omega)$. Also

$$
D U(t, Y(\omega), \omega)(\mathcal{S}(\omega)) \subseteq \mathcal{S}(\theta(t, \omega)), \quad t \geq 0 .
$$

(d) For $\lambda_{i_{0}-1}<\infty, \tilde{\mathcal{U}}(\omega)$ is the set of all $x \in \bar{B}\left(Y(\omega), \rho_{2}(\omega)\right)$ with the property that there is a discrete-time "history" process $y(\cdot, \omega):\{-n: n \geq 0\} \rightarrow H$ such that $y(0, \omega)=x$ and for each integer $n \geq 1$, one has $U(1, y(-n, \omega), \theta(-n, \omega))=y(-(n-1), \omega)$ and

$$
|y(-n, \omega)-Y(\theta(-n, \omega))| \leq \beta_{2}(\omega) e^{-\left(\lambda_{i_{0}-1}-\epsilon_{2}\right) n} .
$$

If $\lambda_{i_{0}-1}=\infty, \tilde{\mathcal{U}}(\omega)$ is the set of all $x \in \bar{B}\left(Y(\omega), \rho_{2}(\omega)\right)$ with the property that there is a discrete-time "history" process $y(\cdot, \omega):\{-n: n \geq 0\} \rightarrow H$ such that $y(0, \omega)=x$ and for each integer $n \geq 1$,

$$
|y(-n, \omega)-Y(\theta(-n, \omega))| \leq \beta_{2}(\omega) e^{-\lambda n},
$$

for any $\lambda \in(0, \infty)$. Furthermore, for each $x \in \tilde{\mathcal{U}}(\omega)$, there is a unique continuoustime "history" process also denoted by $y(\cdot, \omega):(-\infty, 0] \rightarrow H$ such that $y(0, \omega)=x$, $U(t, y(s, \omega), \theta(s, \omega))=y(t+s, \omega)$ for all $s \leq 0,0 \leq t \leq-s$, and

$$
\limsup _{t \rightarrow \infty} \frac{1}{t} \log |y(-t, \omega)-Y(\theta(-t, \omega))| \leq-\lambda_{i_{0}-1}
$$

Each unstable subspace $\mathcal{U}(\omega)$ of the linearized cocycle $(D U(t, Y(\cdot), \cdot), \theta(t, \cdot))$ is tangent at $Y(\omega)$ to $\tilde{\mathcal{U}}(\omega)$, viz. $T_{Y(\omega)} \tilde{\mathcal{U}}(\omega)=\mathcal{U}(\omega)$. In particular, dim $\tilde{\mathcal{U}}(\omega)$ is finite and non-random.

(e) Let $y\left(\cdot, x_{i}, \omega\right), i=1,2$, be the history processes associated with $x_{i}=y\left(0, x_{i}, \omega\right) \in \tilde{\mathcal{U}}(\omega), i=1,2$. Then

$$
\begin{gathered}
\limsup _{t \rightarrow \infty} \frac{1}{t} \log \left[\sup \left\{\frac{\left|y\left(-t, x_{1}, \omega\right)-y\left(-t, x_{2}, \omega\right)\right|}{\left|x_{1}-x_{2}\right|}: x_{1} \neq x_{2}, x_{i} \in \tilde{\mathcal{U}}(\omega), i=1,2\right\}\right] \\
\leq-\lambda_{i_{0}-1} .
\end{gathered}
$$

(f) (Cocycle-invariance of the unstable manifolds):

There exists $\tau_{2}(\omega) \geq 0$ such that

$$
\tilde{\mathcal{U}}(\omega) \subseteq U(t, \cdot, \theta(-t, \omega))(\tilde{\mathcal{U}}(\theta(-t, \omega)))
$$


for all $t \geq \tau_{2}(\omega)$. Also

$$
D U(t, \cdot, \theta(-t, \omega))(\mathcal{U}(\theta(-t, \omega)))=\mathcal{U}(\omega), \quad t \geq 0
$$

and the restriction

$$
D U(t, \cdot, \theta(-t, \omega)) \mid \mathcal{U}(\theta(-t, \omega)): \mathcal{U}(\theta(-t, \omega)) \rightarrow \mathcal{U}(\omega), \quad t \geq 0
$$

is a linear homeomorphism onto.

(g) The submanifolds $\tilde{\mathcal{U}}(\omega)$ and $\tilde{\mathcal{S}}(\omega)$ are transversal, viz.

$$
H=T_{Y(\omega)} \tilde{\mathcal{U}}(\omega) \oplus T_{Y(\omega)} \tilde{\mathcal{S}}(\omega) .
$$

Assume, in addition, that the cocycle $(U, \theta)$ is $C^{\infty}$. Then the local stable and unstable manifolds $\tilde{\mathcal{S}}(\omega), \tilde{\mathcal{U}}(\omega)$ are also $C^{\infty}$.

Fig. 4 summarizes the essential features of the stable manifold theorem:



Fig. 4: The Stable Manifold Theorem 
Before we give a detailed proof of Theorem 2.2.1, we will outline below its basic ingredients.

An outline of the proof of Theorem 2.2.1:

- Since $Y$ is a hyperbolic stationary point of the cocycle $(U, \theta)$ (Definition 2.1.2), then the linearized cocycle satisfies the hypotheses of "perfect versions" of the ergodic theorem and Kingman's subadditive ergodic theorem (Lemma 2.3.1 (ii), (iii) in Section 2.3). These refined versions of the ergodic theorems give invariance of the Oseledec spaces under the continuous-time linearized cocycle (Theorem 2.1.2). Thus the stable/unstable subspaces will serve as tangent spaces to the local stable/unstable manifolds of the non-linear cocycle $(U, \theta)$.

- Define the auxiliary perfect cocycle $(Z, \theta)$ by

$$
Z(t, \cdot, \omega):=U(t,(\cdot)+Y(\omega), \omega)-Y(\theta(t, \omega)), t \in \mathbf{R}^{+}, \omega \in \Omega
$$

This gives a "centering" of the cocycle around the stationary trajectory $Y(\theta(t))$, with the property that $Z$ has a fixed point at $0 \in H$. Employing the continuous-time integrability estimate in Theorem 2.2.1, the perfect ergodic theorem and the perfect subadditive ergodic theorem, the analysis in ([Ru.2], Theorems 5.1 and 6.1) may be extended to obtain local stable/unstable manifolds for the discrete cocycle $(Z(n, \cdot, \omega), \theta(n, \omega))$ near 0 . These manifolds are random objects defined for all $\omega$ which are sampled from a $\theta(t, \cdot)$-invariant sure event in $\Omega$. The translates of these manifolds by the stationary point $Y(\omega)$ correspond to local stable/unstable manifolds for $U(n, \cdot, \omega)$ near $Y(\omega)$. We then interpolate between discrete times and extend the arguments in [Ru.2] further in order to conclude that the above manifolds for the discrete-time cocycle $(U(n, \cdot, \omega), \theta(n, \omega)), n \geq 1$, also serve as local stable/unstable manifolds for the continuous-time cocycle $(U, \theta)$ near $Y$.

- It turns out that the local stable/unstable manifolds are asymptotically invariant under the continuous-time cocycle $(U, \theta)$. For the stable manifolds, the invariance follows by arguments based on (a) a refined version of the perfect subadditive ergodic theorem (Lemma 2.3.2, Section 2.3), and (b) difficult estimates using the integrability property of Theorem 2.2.1 and arguments behind the proofs of Ruelle's Theorems 4.1, 5.1 ([Ru.2]). To establish asymptotic invariance of the local unstable manifolds, we introduce the concept of a stochastic history process for $U$, which compensates for the lack of invertibility of the cocycle. Perfection arguments similar to the above give the invariance. This completes the outline of the proof of Theorem 2.2.1.

A full proof of Theorem 2.2.1 will be given in the next section. The proof is based on a discrete-time version of the theorem given in theorems 5.1, 6.1 [Ru.2]. The extension to continuoustime is done via perfection techniques and interpolation between discrete times. 


\subsection{Proof of the local stable manifold theorem.}

The main objective of this section is to give a proof of Theorem 2.2.1. In particular, we show that the local stable/unstable manifolds for the discrete cocycle are parametrized by sure events which are invariant under the continuous-time shift $\theta(t, \cdot): \Omega \rightarrow \Omega$. This is achieved via a number of computations based on perfection techniques. Excursions of the cocycle between discrete times are controlled by integrability hypothesis on the cocycle $(U, \theta)$ (Theorem 2.2.1).

"Perfect versions" of the ergodic theorem and Kingman's subadditive ergodic theorem will be used to construct the shift-invariant sure events appearing in the statement of the local stable manifold theorem (Theorem 2.2.1). These results are given in Lemmas 2.3.1 and 2.3.2 below.

The following convention will be frequently used throughout the paper:

\section{Definition 2.3.1.}

A family of propositions $\{P(\omega): \omega \in \Omega\}$ is said to hold perfectly in $\omega$ if there is a sure event $\Omega^{*} \in \mathcal{F}$ such that $\theta(t, \cdot)\left(\Omega^{*}\right)=\Omega^{*}$ for all $t \in \mathbf{R}$ and $P(\omega)$ is true for every $\omega \in \Omega^{*}$.

\section{Lemma 2.3.1.}

(i) Let $\Omega_{0} \in \overline{\mathcal{F}}$ be a sure event such that $\theta(t, \cdot)\left(\Omega_{0}\right) \subseteq \Omega_{0}$ for all $t \geq 0$. Then there is a sure event $\Omega_{0}^{*} \in \mathcal{F}$ such that $\Omega_{0}^{*} \subseteq \Omega_{0}$ and $\theta(t, \cdot)\left(\Omega_{0}^{*}\right)=\Omega_{0}^{*}$ for all $t \in \mathbf{R}$.

(ii) Let $h: \Omega \rightarrow \mathbf{R}^{+}$be any function such that there exists an $\overline{\mathcal{F}}$-measurable function $g_{1} \in$ $L^{1}\left(\Omega, \mathbf{R}^{+} ; P\right)$ and a sure event $\Omega_{1} \in \overline{\mathcal{F}}$ such that $\sup _{0 \leq u \leq 1} h(\theta(u, \omega)) \leq g_{1}(\omega)$ for all $\omega \in \Omega_{1}$. Then

$$
\lim _{t \rightarrow \infty} \frac{1}{t} h(\theta(t, \omega))=0
$$

perfectly in $\omega$.

(iii) Suppose $f: \mathbf{R}^{+} \times \Omega \rightarrow \mathbf{R} \cup\{-\infty\}$ is a process such that for each $t \in \mathbf{R}^{+}, f(t, \cdot)$ is $(\overline{\mathcal{F}}, \mathcal{B}(\mathbf{R} \cup\{-\infty\}))$-measurable and the following conditions hold:

(a) There is an $\overline{\mathcal{F}}$-measurable function $g_{2} \in L^{1}\left(\Omega, \mathbf{R}^{+} ; P\right)$ and a sure event $\tilde{\Omega}_{1} \in \overline{\mathcal{F}}$ such that $\left[\sup _{0 \leq u \leq 1} f^{+}(u, \omega)+\sup _{0 \leq u \leq 1} f^{+}(1-u, \theta(u, \omega))\right] \leq g_{2}(\omega)$ for all $\omega \in \tilde{\Omega}_{1}$.

(b) $f\left(t_{1}+t_{2}, \omega\right) \leq f\left(t_{1}, \omega\right)+f\left(t_{2}, \theta\left(t_{1}, \omega\right)\right)$ for all $t_{1}, t_{2} \geq 0$ and all $\omega \in \Omega$.

Then there is a fixed (non-random) number $f^{*} \in \mathbf{R} \cup\{-\infty\}$ such that

$$
\lim _{t \rightarrow \infty} \frac{1}{t} f(t, \omega)=f^{*}
$$

perfectly in $\omega$.

Proof.

Assertion (i) is established in Proposition 2.3 ([M-S.3]).

To prove assertions (ii) and (iii) of the lemma, the reader may adapt the proofs of Lemmas 5 and 7 in [Mo.1] and employ assertion (i) above. Cf. also Lemma 3.3 in [M-S.3]. 
Lemma 2.3.2 below is used to construct the continuous-time shift-invariant sure events which appear in the statement of Theorem 2.2.1. In essence, the lemma is a continuous-time "perfect version" of Ruelle's Corollary A.2 ([Ru.2], p. 288).

\section{Lemma 2.3.2.}

Assume that the process $f: \mathbf{R}^{+} \times \Omega \rightarrow \mathbf{R} \cup\{-\infty\}$ is $\left(\mathcal{B}\left(\mathbf{R}^{+}\right) \otimes \mathcal{F}, \mathcal{B}(\mathbf{R} \cup\{-\infty\})\right)$-measurable and satisfies the following integrability and subadditivity conditions:

(a) $\int_{\Omega}\left[\sup _{0 \leq t_{1}, t_{2} \leq a} f^{+}\left(t_{1}, \theta\left(t_{2}, \omega\right)\right)\right] d P(\omega)<\infty$ for all $a \in(0, \infty)$.

(b) $f\left(t_{1}+t_{2}, \omega\right) \leq f\left(t_{1}, \omega\right)+f\left(t_{2}, \theta\left(t_{1}, \omega\right)\right)$ for all $t_{1}, t_{2} \geq 0$ and all $\omega \in \Omega$.

Then there exists a fixed (non-random) $f^{*} \in \mathbf{R} \cup\{-\infty\}$ such that the following assertions hold perfectly in $\omega$ :

(i) $\lim _{t \rightarrow \infty} \frac{1}{t} f(t, \omega)=f^{*}$.

(ii) Assume $g^{*} \in \mathbf{R}$ is finite and such that $f^{*} \leq g^{*}$. Then for each $\epsilon>0$, there is an $\overline{\mathcal{F}}$ measurable function $K_{\epsilon}: \Omega \rightarrow[0, \infty)$ with the following properties

$$
\begin{aligned}
f(t-s, \theta(s, \omega)) & \leq(t-s) g^{*}+\epsilon t+K_{\epsilon}(\omega), 0 \leq s \leq t<\infty, \\
K_{\epsilon}(\theta(l, \omega)) & \leq K_{\epsilon}(\omega)+\epsilon l, \quad l \in[0, \infty) .
\end{aligned}
$$

Proof.

Applying Lemma 2.3.1 (iii), it is easy to see that there is an $f^{*} \in \mathbf{R} \cup\{-\infty\}$ such that assertion (i) holds for all $\omega$ in a sure event $\Omega_{2} \in \mathcal{F}$ with $\theta(t, \cdot)\left(\Omega_{2}\right)=\Omega_{2}$ for all $t \in \mathbf{R}$. The integrability hypotheses (a) and Lemma 2.3.1 (i) imply that there is a sure event $\Omega_{0} \subseteq \Omega_{2}$ such that $\Omega_{0} \in \mathcal{F}, \theta(t, \cdot)\left(\Omega_{0}\right)=\Omega_{0}$ for all $t \in \mathbf{R}$, and $\sup _{0 \leq t_{1}, t_{2} \leq a} f^{+}\left(t_{1}, \theta\left(t_{2}, \omega\right)\right)<\infty$ for all $a \geq 0$ and all $\omega \in \Omega_{0}$. Let $g^{*}$ be a finite number in $\left[f^{*}, \infty\right)$. Define the non-negative process $g: \mathbf{R}^{+} \times \Omega \rightarrow \mathbf{R}^{+}$ by

$$
g(t, \omega):= \begin{cases}\max \left\{f(t, \omega)-t g^{*}, 0\right\}, & t \geq 0, \omega \in \Omega_{0}, \\ 0 & t \geq 0, \omega \notin \Omega_{0} .\end{cases}
$$

Then $g$ is $\left(\mathcal{B}\left(\mathbf{R}^{+}\right) \otimes \mathcal{F}, \mathcal{B}\left(\mathbf{R}^{+}\right)\right)$-measurable and satisfies conditions (a) and (b).

Now consider the non-negative process $g^{\prime}: \mathbf{R}^{+} \times \Omega \rightarrow \mathbf{R}^{+}$defined by

$$
g^{\prime}(t, \omega):=\sup _{0 \leq s \leq t}[g(s, \omega)+g(t-s, \theta(s, \omega))], \quad t \geq 0, \omega \in \Omega
$$

Observe that the projection of a $\left(\mathcal{B}\left(\mathbf{R}^{+}\right) \otimes \mathcal{F}\right)$-measurable set is $\overline{\mathcal{F}}$-measurable ([Co], p. 281). Therefore, $g^{\prime}$ satisfies all the hypotheses of Lemma 2.3.1 (iii). This gives a non-negative $g^{\prime *}$ such that $\lim _{t \rightarrow \infty} \frac{1}{t} g^{\prime}(t, \omega)=g^{\prime *}$ for all $\omega$ in a sure event $\Omega_{3} \in \mathcal{F}$, with $\theta(t, \cdot)\left(\Omega_{3}\right)=\Omega_{3}$ for all $t \in \mathbf{R}$.

We will show next the following convergence in probability:

$$
\lim _{t \rightarrow \infty} \frac{1}{t} \sup _{0 \leq s \leq t} g(t-s, \theta(s, \cdot))=0 .
$$


To do this, observe that the process $h: \mathbf{R}^{+} \times \Omega \rightarrow \mathbf{R}, h(t, \omega):=g(t, \theta(-t, \omega)), t \in \mathbf{R}^{+}, \omega \in \Omega$, satisfies the conditions of Lemma 2.3.1 (iii). Therefore

$$
\lim _{t \rightarrow \infty} \frac{1}{t} h(t, \cdot)=0
$$

almost surely and hence in probability. Pick $\delta, t_{0}>0$ such that $P\left(\frac{1}{t} h(t, \cdot) \geq \delta\right)<\delta$ for all $t \geq t_{0}$. Let $t \geq t_{0}$. Then

$$
\begin{aligned}
\sup _{0 \leq s \leq t} \frac{1}{t} g(t-s, \theta(s, \omega)) & \leq \sup _{0 \leq s \leq t-t_{0}} \frac{1}{t} g(t-s, \theta(s, \omega))+\sup _{t-t_{0} \leq s \leq t} \frac{1}{t} g(t-s, \theta(s, \omega)) \\
& \leq \sup _{0 \leq s \leq t-t_{0}} \frac{1}{t} g(t-s, \theta(-(t-s), \theta(t, \omega)))+\sup _{t-t_{0} \leq s \leq t} \frac{1}{t} g(t-s, \theta(s, \omega)) .
\end{aligned}
$$

By condition (a), the second term in the right hand side of the last inequality converges to zero in probability. The probability that the first term is less than or equal to $\delta$ is at least $1-\delta$. Hence (2.3.1) holds.

It follows easily from (2.3.1) that $g^{\prime *}=0$. This implies that assertion (i) holds for all $\omega$ in a sure event $\Omega_{4} \in \mathcal{F}$ with $\Omega_{4} \subseteq \Omega_{0} \cap \Omega_{3}$ and $\theta(t, \cdot)\left(\Omega_{4}\right)=\Omega_{4}$ for all $t \in \mathbf{R}$. To complete the proof of assertion (ii), let $\epsilon>0$ and define the $\left(\overline{\mathcal{F}}, \mathcal{B}\left(\mathbf{R}^{+}\right)\right)$-measurable function $K_{\epsilon}: \Omega_{4} \rightarrow[0, \infty)$ by

$$
K_{\epsilon}(\omega):=\sup _{0 \leq s \leq t<\infty}[g(t-s, \theta(s, \omega))-\epsilon t]
$$

for all $\omega \in \Omega_{4}$. This completes the proof of the lemma.

Lemma 2.3.3 below is essentially a "perfect version" of Proposition 3.2 in [Ru.2], p. 257. Our Lemma 2.3.2 plays a crucial role in the proof of Lemma 2.3.3. In the statement of the lemma, we will use $\mathcal{B}_{s}(L(H))$ to denote the Borel $\sigma$-algebra on $L(H)$ generated by the strong topology on $L(H)$, viz. the smallest topology on $L(H)$ for which all evaluations $L(H) \ni A \mapsto A(z) \in H, z \in H$, are continuous.

\section{Lemma 2.3.3.}

Suppose $\left(T^{t}(\omega), \theta(t, \omega)\right), t \geq 0$, is a perfect cocycle of bounded linear operators in $H$ satisfying the following hypotheses:

(i) The process $\mathbf{R}^{+} \times \Omega \ni(t, \omega) \mapsto T^{t}(\omega) \in L(H)$ is $\left(\mathcal{B}\left(\mathbf{R}^{+}\right) \otimes \mathcal{F}, \mathcal{B}_{s}(L(H))\right)$-measurable.

(ii) The map $\mathbf{R}^{+} \times \Omega \ni(t, \omega) \mapsto \theta(t, \omega) \in \Omega$ is $\left(\mathcal{B}\left(\mathbf{R}^{+}\right) \otimes \mathcal{F}, \mathcal{F}\right)$-measurable, and is a group of ergodic P-preserving transformations on $(\Omega, \mathcal{F}, P)$.

(iii) $E \sup _{0 \leq t_{1}, t_{2} \leq a} \log ^{+}\left\|T^{t_{2}}\left(\theta\left(t_{1}, \cdot\right)\right)\right\|_{L(H)}<\infty$ for any finite $a>0$.

(iv) For each $t>0, T^{t}(\omega)$ is compact, perfectly in $\omega$.

(v) For any $u \in H$, the map $[0, \infty) \ni t \mapsto T^{t}(\omega)(u) \in H$ is continuous, perfectly in $\omega$.

Let $\left\{\cdots<\lambda_{i+1}<\lambda_{i}<\cdots<\lambda_{2}<\lambda_{1}\right\}$ be the Lyapunov spectrum of $\left(T^{t}(\omega), \theta(t, \omega)\right)$, with Oseledec spaces

$$
\cdots E_{i+1}(\omega) \subset E_{i}(\omega) \subset \cdots \subset E_{2}(\omega) \subset E_{1}(\omega)=H .
$$


Let $j_{0} \geq 1$ be any fixed integer with $\lambda_{j_{0}}>-\infty$. Let the integer function $r:\{1,2, \cdots, Q\} \rightarrow$ $\left\{1,2, \cdots, j_{0}\right\}$ "count" the multiplicities of the Lyapunov exponents in the sense that $r(1)=1, r(Q)=$ $j_{0}$, and for each $1 \leq i \leq j_{0}$, the number of integers in $r^{-1}(i)$ is the multiplicity of $\lambda_{i}$. Set $V_{n}(\omega):=E_{j_{0}+1}(\theta(n, \omega)), n \geq 0$.

Then the sequence $T_{n}(\omega):=T^{1}(\theta((n-1), \omega)), n \geq 1$, satisfies Condition (S) of ([Ru.2], pp. 256-257) perfectly in $\omega$ with $Q=$ codim $E_{j_{0}+1}(\omega)$. In particular, there is an $\mathcal{F}$-measurable set of $Q$ orthonormal vectors $\left\{\xi_{0}^{(1)}(\omega), \cdots, \xi_{0}^{(Q)}(\omega)\right\}$ such that $\xi_{0}^{(k)}(\omega) \in\left[E_{r(k)}(\omega) \backslash E_{r(k)+1}(\omega)\right]$ for $k=1, \cdots, Q$, perfectly in $\omega$, and satisfying the following properties:

$$
\begin{aligned}
& \text { Set } \xi_{t}^{(k)}(\omega):=\frac{T^{t}(\omega)\left(\xi_{0}^{(k)}(\omega)\right)}{\left|T^{t}(\omega)\left(\xi_{0}^{(k)}(\omega)\right)\right|} \text {, and for any } u \in H, \text { write } \\
& \quad u=\sum_{k=1}^{Q} u_{t}^{(k)}(\omega) \xi_{t}^{(k)}(\omega)+u_{t}^{(Q+1)}(\omega), \quad u_{t}^{(Q+1)}(\omega) \in V_{0}(\theta(t, \omega)), \omega \in \Omega .
\end{aligned}
$$

Then for any $\epsilon>0$, there is an $\overline{\mathcal{F}}$-measurable random constant $D_{\epsilon}(\omega)>0$ such that the following inequalities hold perfectly in $\omega$ :

$$
\begin{aligned}
\left|u_{t}^{(k)}(\omega)\right| & \leq D_{\epsilon}(\omega) e^{\epsilon t}|u| \\
\left|u_{t}^{(Q+1)}(\omega)\right| & \leq D_{\epsilon}(\omega) e^{\epsilon t}|u| \\
D_{\epsilon}(\theta(l, \omega)) & \leq D_{\epsilon}(\omega) e^{\epsilon l}
\end{aligned}
$$

for all $t \geq 0,1 \leq k \leq Q$ and for all $l \in[0, \infty)$.

Furthermore, all the random constants in Ruelle's Condition (S) ([Ru.2], pp. 256-257) may be chosen to be $\overline{\mathcal{F}}$-measurable in $\omega$.

Proof.

Our proof runs along similar lines to that of Proposition 3.2 in [Ru.2]: However, one has to maintain the non-trivial requirement that all relevant arguments hold perfectly in $\omega$.

It is assumed throughout this proof that the reader is familiar with Ruelle's conditions (S): (S1)-(S4) as spelled out in ([Ru.2], pp. 256-257).

Observe first that $T_{n}(\omega)$ satisfies (S1) perfectly in $\omega$. This holds because of (iii), the perfect cocycle property, Lemma 2.3.1 and the proof of Theorem 4 ([Mo.1]). Note that, by the ordering of the fixed Lyapunov spectrum, relation (3.4) of [Ru.2] holds perfectly. Denote by $\Omega^{*}$ the $\theta(t, \cdot)$ invariant sure event where (S1) holds. Using ergodicity of $\theta$ and the fact that $\operatorname{codim} V_{0}(\omega)=Q$, for all $\omega \in \Omega^{*}$, it follows that codim $V_{n}(\omega)=\operatorname{codim} E_{j_{0}+1}(\theta(n, \omega))=Q$. Therefore, (S2) is satisfied for all $\omega \in \Omega^{*}$.

We next prove that (S3) holds perfectly. To do this, we will prove the stronger assertion that the continuous-time cocycle $\left(T^{t}(\omega), \theta(t, \omega)\right)$ satisfies (S3) perfectly in $\omega$. Set $\hat{T}^{t}(\omega):=$ $T^{t}(\omega) \mid V_{0}(\omega), \omega \in \Omega^{*}, t \geq 0$. Hence $\hat{T}^{t}(\omega)\left(V_{0}(\omega)\right) \subseteq V_{0}(\theta(t, \omega))$, and the following cocycle identity

$$
\hat{T}^{t_{1}+t_{2}}(\omega)=\hat{T}^{t_{2}}\left(\theta\left(t_{1}, \omega\right)\right) \circ \hat{T}^{t_{1}}(\omega)
$$


holds for all $\omega \in \Omega^{*}, t \geq 0$. Denote $F_{t}(\omega):=\log \left\|\hat{T}^{t}(\omega)\right\|, \omega \in \Omega^{*}, t \geq 0$. Hypothesis (iii) of the lemma easily implies that $E \sup _{0 \leq t_{1}, t_{2} \leq a} F_{t_{2}}^{+}\left(\theta\left(t_{1}, \cdot\right)\right)<\infty$ for any finite $a>0$. Furthermore, $\left(F_{t}(\omega), \theta(t, \omega)\right)$ is perfectly subadditive because of the above cocycle identity. Applying Lemma 2.3.1, we obtain a fixed number $F^{*} \in \mathbf{R} \cup\{-\infty\}$ such that

$$
\lim _{t \rightarrow \infty} \frac{1}{t} F_{t}(\omega)=F^{*}
$$

perfectly in $\omega$. Suppose $S=j_{0}$. When $\lambda_{j_{0}+1}>-\infty$, set $\mu^{(S+1)}:=\lambda_{j_{0}+1}$; and when $\lambda_{j_{0}+1}=-\infty$, take $\mu^{(S+1)}$ to be any fixed number in $\left(-\infty, \lambda_{j_{0}}\right)$. Using (3.5), p. 257 of [Ru.2], it follows that $F^{*} \leq \mu^{(S+1)}$. Suppose $\epsilon>0$ and $\lambda_{j_{0}+1}>-\infty$. Then by Lemma 2.3.2(ii), there is an $\overline{\mathcal{F}}$-measurable function $K_{\epsilon}: \Omega \rightarrow[0, \infty)$ such that

$$
\log \left\|\hat{T}^{t-s}(\theta(s, \omega))\right\| \leq(t-s) \mu^{(S+1)}+\epsilon t+K_{\epsilon}(\omega), \quad 0 \leq s \leq t<\infty,
$$

and

$$
K_{\epsilon}(\theta(l, \omega)) \leq K_{\epsilon}(\omega)+\epsilon l, \quad l \in[0, \infty),
$$

perfectly in $\omega$. When $\lambda_{j_{0}+1}=-\infty$, the inequality (2.3.2) holds where $\mu^{(S+1)}$ is replaced by any (finite) number in $\left(-\infty, \lambda_{j_{0}}\right)$. Now let $m, n$ be positive integers such that $m<n$. In (2.3.2), replace $t$ by $n$ and $s$ by $m+1$ to see that $T_{n}(\omega), n \geq 1$, satisfies (S3) perfectly in $\omega$.

The rest of this proof will now focus on showing that the sequence $T_{n}(\omega), n \geq 1$, also satisfies Ruelle's condition (S4) perfectly in $\omega$. Indeed, we will establish the stronger statement that the continuous-time cocycle $\left(T^{t}(\omega), \theta(t, \omega)\right)$ satisfies (S4) perfectly in $\omega$. Using the orthogonal decomposition $H=V_{0}(\theta(t, \omega)) \oplus V_{0}(\theta(t, \omega))^{\perp}$, write

$$
T^{t}(\omega)(\xi)=\check{T}^{t}(\omega)(\xi)+\tilde{T}^{t}(\omega)(\xi), \quad \xi \in H, t \geq 0, \omega \in \Omega^{*} .
$$

That is, $\tilde{T}^{t}(\omega)(\xi) \in V_{0}(\theta(t, \omega))$ and $\check{T}^{t}(\omega)(\xi) \in V_{0}(\theta(t, \omega))^{\perp}$ are the orthogonal projections of $T^{t}(\omega)(\xi)$ on $V_{0}(\theta(t, \omega))$ and $V_{0}(\theta(t, \omega))^{\perp}$, respectively. Thus (2.3.3) defines a family of continuous linear operators $\check{T}^{t}(\omega): H \rightarrow V_{0}(\theta(t, \omega))^{\perp} \subseteq H, \tilde{T}^{t}(\omega): H \rightarrow V_{0}(\theta(t, \omega)) \subseteq H, t \geq 0$. We now show that the family $\left(\check{T}^{t}(\omega), \theta(t, \omega)\right), \omega \in \Omega$, satisfies the perfect cocycle property in $L(H)$. To prove this, we fix any $\omega \in \Omega$. Then by the cocycle property of $\left(T^{t}(\omega), \theta(t, \omega)\right)$ and $(2.3 .3)$, we obtain

$$
\begin{aligned}
T^{t_{1}+t_{2}}(\omega)(\xi)= & T^{t_{2}}\left(\theta\left(t_{1}, \omega\right)\right)\left[T^{t_{1}}(\omega)(\xi)\right] \\
= & \check{T}^{t_{2}}\left(\theta\left(t_{1}, \omega\right)\right)\left[\check{T}^{t_{1}}(\omega)(\xi)\right]+\check{T}^{t_{2}}\left(\theta\left(t_{1}, \omega\right)\right)\left[\tilde{T}^{t_{1}}(\omega)(\xi)\right]+\tilde{T}^{t_{2}}\left(\theta\left(t_{1}, \omega\right)\right)\left[\check{T}^{t_{1}}(\omega)(\xi)\right] \\
& \quad+\tilde{T}^{t_{2}}\left(\theta\left(t_{1}, \omega\right)\right)\left[\tilde{T}^{t_{1}}(\omega)(\xi)\right] .
\end{aligned}
$$

for all $t_{1}, t_{2} \geq 0, \xi \in H$. Furthermore, $\check{T}^{t}(\omega)(\xi)=0$ for all $\xi \in V_{0}(\omega)$, because $V_{0}(\omega)$ is invariant under the cocycle $\left(T^{t}(\omega), \theta(t, \omega)\right)$. Thus, $\check{T}^{t_{2}}\left(\theta\left(t_{1}, \omega\right)\right)\left[\tilde{T}^{t_{1}}(\omega)(\xi)\right]=0$ for all $\xi \in H$, and $(2.3 .4)$ yields

$$
\begin{aligned}
& T^{t_{1}+t_{2}}(\omega)(\xi) \\
& \quad=\check{T}^{t_{2}}\left(\theta\left(t_{1}, \omega\right)\right)\left[\check{T}^{t_{1}}(\omega)(\xi)\right]+\tilde{T}^{t_{2}}\left(\theta\left(t_{1}, \omega\right)\right)\left[\check{T}^{t_{1}}(\omega)(\xi)\right]+\tilde{T}^{t_{2}}\left(\theta\left(t_{1}, \omega\right)\right)\left[\tilde{T}^{t_{1}}(\omega)(\xi)\right] .
\end{aligned}
$$


Now

$$
T^{t_{1}+t_{2}}(\omega)(\xi)=\check{T}^{t_{1}+t_{2}}(\omega)(\xi)+\tilde{T}^{t_{1}+t_{2}}(\omega)(\xi)
$$

for all $\xi \in H$. In the right hand side of (2.3.5), the first term belongs to $V_{0}\left(\theta\left(t_{1}+t_{2}, \omega\right)\right)^{\perp}$, while the second two terms belong to $V_{0}\left(\theta\left(t_{1}+t_{2}, \omega\right)\right)$. So by uniqueness of the orthogonal decomposition, it follows from (2.3.6) and (2.3.5) that

$$
\check{T}^{t_{1}+t_{2}}(\omega)(\xi)=\check{T}^{t_{2}}\left(\theta\left(t_{1}, \omega\right)\right)\left[\check{T}^{t_{1}}(\omega)(\xi)\right]
$$

for all $\xi \in H$. Hence $\left(\check{T}^{t}(\omega), \theta(t, \omega)\right)$ is a perfect cocycle in $L(H)$.

We next verify that both cocycles $\left(T^{t}(\omega), \theta(t, \omega)\right)$ and $\left(\check{T}^{t}(\omega), \theta(t, \omega)\right)$ satisfy the conditions of the perfect Oseledec theorem (Theorem 2.1.1). To see this, note that

$$
E \sup _{0 \leq t_{1}, t_{2} \leq a} \log ^{+}\left\|\check{T}^{t_{2}}\left(\theta\left(t_{1}, \cdot\right)\right)\right\|_{L(H)}<\infty
$$

for any finite $a>0$. This follows immediately from the integrability property (iii) of the lemma. Now apply Theorem 2.1.1 to $\left(T^{t}(\omega), \theta(t, \omega)\right)$ and $\left(\check{T}^{t}(\omega), \theta(t, \omega)\right)$. This gives the following limits

$$
\lim _{t \rightarrow \infty} \frac{1}{t} \log \left|\check{T}^{t}(\omega)(\xi)\right|=\check{l}_{\xi}, \quad \lim _{t \rightarrow \infty} \frac{1}{t} \log \left|T^{t}(\omega)(\xi)\right|=l_{\xi}
$$

perfectly in $\omega$ for all $\xi \in H$, with $l_{\xi}, \check{l}_{\xi}$ fixed numbers in $\mathbf{R} \cup\{-\infty\}$. We now apply (3.6) in ([Ru.2], p. 259) to obtain

$$
\check{l}_{\xi}=\lim _{n \rightarrow \infty} \frac{1}{n} \log \left|\check{T}^{n}(\omega)(\xi)\right|=\lim _{n \rightarrow \infty} \frac{1}{n} \log \left|T^{n}(\omega)(\xi)\right|=l_{\xi}
$$

for a.a. $\omega$ and all $\xi \in H \backslash V_{0}(\omega)$. Therefore the equality

$$
\lim _{t \rightarrow \infty} \frac{1}{t} \log \left|\check{T}^{t}(\omega)(\xi)\right|=\lim _{t \rightarrow \infty} \frac{1}{t} \log \left|T^{t}(\omega)(\xi)\right|
$$

holds perfectly in $\omega$ for all $\xi \in H \backslash V_{0}(\omega)$. Hence, relation (3.6) in ([Ru.2], p. 259) may be replaced by the continuous-time "perfect" relation

$$
\lim _{t \rightarrow \infty} \frac{1}{t} \log \frac{\left|\check{T}^{t}(\omega)(\xi)\right|}{\left|T^{t}(\omega)(\xi)\right|}=0
$$

for all $\xi \in H \backslash V_{0}(\omega)$.

By ([C-V], Theorem III.6, p. 65) and Gram-Schmidt orthogonalization, we may select a set of $Q, \mathcal{F}$-measurable, orthonormal vectors $\left\{\xi_{0}^{(1)}(\omega), \cdots, \xi_{0}^{(Q)}(\omega)\right\}$ such that $\xi_{0}^{(k)}(\omega) \in\left[E_{r(k)}(\omega) \backslash E_{r(k)+1}(\omega)\right] \cap$ $V_{0}(\omega)^{\perp}$ for $k=1, \cdots, Q$, perfectly in $\omega$. In the argument in [Ru.2], p. 259, replace (3.6) by (2.3.9) above, $n$ by $t, \xi_{n}^{(k)}$ by $\xi_{t}^{(k)}(\omega):=\frac{T^{t}(\omega)\left(\xi_{0}^{(k)}(\omega)\right)}{\left|T^{t}(\omega)\left(\xi_{0}^{(k)}(\omega)\right)\right|}, V_{n}$ by $V_{0}(\theta(t, \omega))$, and $\eta_{n}^{(k)}$ by $\eta_{t}^{(k)}(\omega):=$ $\frac{\check{T}^{t}(\omega)\left(\xi_{0}^{(k)}(\omega)\right)}{\left|T^{t}(\omega)\left(\xi_{0}^{(k)}(\omega)\right)\right|}$. Therefore for $u \in H$, we write

$$
u=\sum_{k=1}^{Q} u_{t}^{(k)}(\omega) \xi_{t}^{(k)}(\omega)+u_{t}^{(Q+1)}(\omega), \quad u_{t}^{(Q+1)}(\omega) \in V_{0}(\theta(t, \omega)),
$$


perfectly in $\omega$ for all $t \geq 0$. Furthermore, as in [Ru.2], p. 259, (2.3.9) implies that

$$
\lim _{t \rightarrow \infty} \frac{1}{t} \log \left|\operatorname{det}\left(\eta_{t}^{(1)}(\omega), \cdots, \eta_{t}^{(Q)}(\omega)\right)\right|=0
$$

perfectly in $\omega$.

It remains to prove that for each $\epsilon>0$, there is an $\overline{\mathcal{F}}$-measurable non-negative function $D_{\epsilon}: \Omega \rightarrow(0, \infty)$ such that the following inequalities

$$
\left.\begin{array}{rl}
\left|u_{t}^{(k)}(\omega)\right| & \leq D_{\epsilon}(\omega) e^{\epsilon t}|u| \\
\left|u_{t}^{(Q+1)}(\omega)\right| & \leq D_{\epsilon}(\omega) e^{\epsilon t}|u| \\
D_{\epsilon}(\theta(l, \omega)) & \leq D_{\epsilon}(\omega) e^{\epsilon l}
\end{array}\right\}
$$

hold perfectly in $\omega$, for all $t \geq 0,1 \leq k \leq Q$ and for all $l \in[0, \infty)$.

In order to establish the inequalities (3.12), we define

$$
D_{\epsilon}(\omega):=1+Q \cdot \sup _{0 \leq s \leq t<\infty} e^{-\epsilon t}\left|\operatorname{det}\left(\eta_{t-s}^{(1)}(\theta(s, \omega)), \eta_{t-s}^{(2)}(\theta(s, \omega)), \cdots, \eta_{t-s}^{(Q)}(\theta(s, \omega))\right)\right|^{-1}
$$

perfectly in $\omega$.

First of all we must show that $D_{\epsilon}(\omega)$ is finite perfectly in $\omega$. Surprisingly, this will require some work. Let $0 \leq s \leq t$. Observe that the determinant of the linear operator $\check{T}^{t-s}(\theta(s, \omega))$ is given by $\frac{\left|\wedge_{k=1}^{Q} \check{T}^{t-s}(\theta(s, \omega))\left(v_{k}\right)\right|}{\left|\wedge_{k=1}^{Q} v_{k}\right|}$ for any choice of basis $\left\{v_{1}, \cdots, v_{Q}\right\}$ in $V_{0}(\theta(s, \omega))^{\perp}$. Therefore, the following inequalities hold perfectly in $\omega$ :

$$
\begin{aligned}
\mid \operatorname{det}\left(\eta_{t-s}^{(1)}\right. & \left.(\theta(s, \omega)), \cdots, \eta_{t-s}^{(Q)}(\theta(s, \omega))\right)\left.\right|^{-1} \\
& =\frac{\Pi_{k=1}^{Q}\left|T^{t-s}(\theta(s, \omega))\left(\xi_{0}^{(k)}(\theta(s, \omega))\right)\right|}{\mid \operatorname{det}\left(\check{T}^{t-s}(\theta(s, \omega))\left(\xi_{0}^{(1)}(\theta(s, \omega))\right), \cdots, \check{T}^{t-s}(\theta(s, \omega))\left(\xi_{0}^{(Q)}(\theta(s, \omega))\right) \mid\right.} \\
& =\frac{\Pi_{k=1}^{Q}\left[\left|T^{t-s}(\theta(s, \omega))\left(\xi_{0}^{(k)}(\theta(s, \omega))\right)\right| \cdot\left|\wedge_{k=1}^{Q}\left[\check{T}^{s}(\omega)\left(\xi_{0}^{(k)}(\omega)\right)\right]\right|\right.}{\left|\operatorname{det}\left(\check{T}^{t-s}(\theta(s, \omega))\left(\check{T}^{s}(\omega)\left(\xi_{0}^{(1)}(\omega)\right)\right), \cdots, \check{T}^{t-s}(\theta(s, \omega))\left(\check{T}^{s}(\omega)\left(\xi_{0}^{(Q)}(\omega)\right)\right)\right)\right|} \\
& \leq \frac{\Pi_{k=1}^{Q}\left[\mid T^{t-s}(\theta(s, \omega))\left(\xi_{0}^{(k)}(\theta(s, \omega))|\cdot| \check{T}^{s}(\omega)\left(\xi_{0}^{(k)}(\omega)\right) \mid\right]\right.}{\mid \operatorname{det}\left(\check{T}^{t}(\omega)\left(\xi_{0}^{(1)}(\omega)\right), \cdots, \check{T}^{t}(\omega)\left(\xi_{0}^{(Q)}(\omega)\right) \mid\right.} \\
& =\frac{\Pi_{k=1}^{Q}\left[\left|T^{t-s}(\theta(s, \omega))\left(\xi_{0}^{(k)}(\theta(s, \omega))\right) \cdot\right| \check{T}^{s}(\omega)\left(\xi_{0}^{(k)}(\omega)\right) \mid\right]}{\left\|\left[\check{T}^{t}(\omega) \mid V_{0}(\omega) \perp\right]^{\wedge Q}\right\|} \\
& \leq \frac{\left\|T^{t-s}(\theta(s, \omega))\right\|^{Q} \cdot\left\|\check{T}^{s}(\omega)\right\|^{Q}}{\left\|\left[\check{T}^{t}(\omega) \mid V_{0}(\omega)^{\perp}\right]^{\wedge Q}\right\|}
\end{aligned}
$$

By the integrability condition (iii), it follows that

$$
\sup _{0 \leq s \leq t \leq a}\left\|T^{t-s}(\theta(s, \omega))\right\|^{Q} \cdot\left\|\check{T}^{s}(\omega)\right\|^{Q}<\infty
$$

perfectly in $\omega$ for any finite $a>0$. 
We now prove that for each finite $a>0$,

$$
\sup _{0 \leq s \leq t \leq a}\left|\operatorname{det}\left(\eta_{t-s}^{(1)}(\theta(s, \omega)), \cdots, \eta_{t-s}^{(Q)}(\theta(s, \omega))\right)\right|^{-1}<\infty
$$

perfectly in $\omega$. To see this, define the compact set

$$
S(\omega):=\left\{\left(t, v_{1}, \cdots, v_{Q}\right): t \in[0, a], v_{k} \in V_{0}(\omega)^{\perp},\left|v_{k}\right|=1,<v_{k}, v_{l}>=0,1 \leq k<l \leq Q\right\} .
$$

for $\omega \in \Omega$. Thus (2.3.16) will hold if we prove that

$$
\inf _{\left(t, v_{1}, \cdots, v_{Q}\right) \in S(\omega)}\left|\wedge_{k=1}^{Q}\left[\check{T}^{t}(\omega)\left(v_{k}\right)\right]\right|>0
$$

perfectly in $\omega$.

To prove (2.3.17), we observe that each map $\check{T}^{t}(\omega) \mid V_{0}(\omega)^{\perp}: V_{0}(\omega)^{\perp} \rightarrow V_{0}(\theta(t, \omega))^{\perp}$ is injective for each $t \geq 0$ perfectly in $\omega$. This is an easy consequence of the cocycle property and the fact that $\lambda_{j_{0}}>-\infty$. In fact,

$$
\left|\wedge_{k=1}^{Q}\left[\check{T}^{t}(\omega)\left(v_{k}\right)\right]\right|>0
$$

for all $\left(t, v_{1}, \cdots, v_{Q}\right) \in S(\omega)$. Furthermore, the map

$$
[0, a] \times\left[V_{0}(\omega)^{\perp}\right]^{Q} \ni\left(t, v_{1}, \cdots, v_{Q}\right) \mapsto\left|\wedge_{k=1}^{Q}\left[\check{T}^{t}(\omega)\left(v_{k}\right)\right]\right| \in[0, \infty)
$$

is jointly continuous, by hypothesis (v) of the lemma. By compactness of $S(\omega)$, (2.3.18) implies (2.3.17). Therefore, (2.3.16) follows from (2.3.15) and (2.3.17).

The following convergence

$$
\lim _{t \rightarrow \infty} \frac{1}{t} \log \sup _{0 \leq s \leq t}\left|\operatorname{det}\left(\eta_{t-s}^{(1)}(\theta(s, \omega)), \cdots, \eta_{t-s}^{(Q)}(\theta(s, \omega))\right)\right|^{-1}=0
$$

holds perfectly in $\omega$. To prove this convergence, note that (2.3.14) implies the following estimate

$$
\begin{aligned}
\mid \operatorname{det}\left(\eta_{t-s}^{(1)}(\theta(s, \omega)), \cdots\right. & \left., \eta_{t-s}^{(Q)}(\theta(s, \omega))\right)\left.\right|^{-1} \\
& \leq \frac{\Pi_{k=1}^{Q}\left\{\|\left[T^{t-s}(\theta(s, \omega)) \mid E_{r(k)}(\theta(s, \omega)]\|\cdot\|\left[\check{T}^{s}(\omega) \mid E_{r(k)}(\omega)\right] \|\right\}\right.}{\left\|\left[\check{T}^{t}(\omega) \mid V_{0}(\omega)^{\perp}\right]^{\wedge Q}\right\|}
\end{aligned}
$$

for $0 \leq s \leq t$ perfectly in $\omega$. Let $\epsilon>0$ be arbitrary. Taking $\frac{1}{t} \log \sup _{0 \leq s \leq t}$ on both sides of the above inequality and applying Lemma 2.3.2(ii) yields the following inequalities

$$
\begin{aligned}
& \frac{1}{t} \log \sup _{0 \leq s \leq t} \mid\left.\operatorname{det}\left(\eta_{t-s}^{(1)}(\theta(s, \omega)), \cdots, \eta_{t-s}^{(Q)}(\theta(s, \omega))\right)\right|^{-1} \\
& \leq \frac{1}{t} \sup _{0 \leq s \leq t}\left\{\sum_{k=1}^{Q}\left(\log \|\left[T^{t-s}(\theta(s, \omega)) \mid E_{r(k)}(\theta(s, \omega)]\|+\log \|\left[\check{T}^{s}(\omega) \mid E_{r(k)}(\omega)\right] \|\right)\right\}\right. \\
& \quad-\frac{1}{t} \log \left\|\left[\check{T}^{t}(\omega) \mid V_{0}(\omega)^{\perp}\right]^{\wedge Q}\right\|
\end{aligned}
$$




$$
\begin{aligned}
\leq \frac{1}{t} \sup _{0 \leq s \leq t}\left\{\sum_{k=1}^{Q}(t-s) \lambda_{r(k)}\right. & \left.+\epsilon t+K_{\epsilon}^{1}(\omega)+\sum_{k=1}^{Q} s \lambda_{r(k)}+\epsilon s+K_{\epsilon}^{2}(\omega)\right\} \\
& -\frac{1}{t} \log \left\|\left[\check{T}^{t}(\omega) \mid V_{0}(\omega)^{\perp}\right]^{\wedge Q}\right\| \\
= & \sum_{k=1}^{Q} \lambda_{r(k)}+2 \epsilon+\frac{1}{t}\left[K_{\epsilon}^{1}(\omega)+K_{\epsilon}^{2}(\omega)\right]-\frac{1}{t} \log \left\|\left[\check{T}^{t}(\omega) \mid V_{0}(\omega)^{\perp}\right]^{\wedge Q}\right\|, \quad t>0,
\end{aligned}
$$

perfectly in $\omega$, with $K_{\epsilon}^{i}(\omega), i=1,2$, finite positive random constants independent of $t$. Therefore, the above inequality implies that

$$
\begin{aligned}
& \limsup _{t \rightarrow \infty} \frac{1}{t} \log \sup _{0 \leq s \leq t}\left|\operatorname{det}\left(\eta_{t-s}^{(1)}(\theta(s, \omega)), \cdots, \eta_{t-s}^{(Q)}(\theta(s, \omega))\right)\right|^{-1} \\
& \leq \sum_{k=1}^{Q} \lambda_{r(k)}+2 \epsilon-\liminf _{t \rightarrow \infty} \frac{1}{t} \log \left\|\left[\check{T}^{t}(\omega) \mid V_{0}(\omega)^{\perp}\right]^{\wedge Q}\right\| \\
&=\sum_{k=1}^{Q} \lambda_{r(k)}+2 \epsilon-\sum_{k=1}^{Q} \lambda_{r(k)} \\
&=2 \epsilon .
\end{aligned}
$$

Since $\epsilon>0$ is arbitrary, then

$$
\limsup _{t \rightarrow \infty} \frac{1}{t} \log \sup _{0 \leq s \leq t}\left|\operatorname{det}\left(\eta_{t-s}^{(1)}(\theta(s, \omega)), \cdots, \eta_{t-s}^{(Q)}(\theta(s, \omega))\right)\right|^{-1} \leq 0
$$

perfectly in $\omega$. The convergence (2.3.11) immediately implies the inequality

$$
\begin{aligned}
& \liminf _{t \rightarrow \infty} \frac{1}{t} \log \sup _{0 \leq s \leq t} \mid\left.\operatorname{det}\left(\eta_{t-s}^{(1)}(\theta(s, \omega)), \cdots, \eta_{t-s}^{(Q)}(\theta(s, \omega))\right)\right|^{-1} \\
& \geq \liminf _{t \rightarrow \infty} \frac{1}{t} \log \left|\operatorname{det}\left(\eta_{t}^{(1)}(\omega), \cdots, \eta_{t}^{(Q)}(\omega)\right)\right|^{-1}=0
\end{aligned}
$$

Thus (2.3.19) follows from (2.3.20) and (2.3.21).

From (2.3.16), (2.3.19) and (2.3.13), we conclude that $D_{\epsilon}(\omega)$ is finite perfectly in $\omega$.

From the Definition 2.3.13 of $D(\omega)$, one immediately gets the last inequality in (2.3.12).

It remains to show the first two inequalities in (2.3.12). In the right hand side of (2.3.10), we look at the terms

$$
\check{u}(\omega)=\sum_{k=1}^{Q} u_{t}^{(k)}(\omega) \eta_{t}^{(k)}(\omega), \quad u \in H, t \geq 0,
$$

where $\check{u}(\omega), \eta_{t}^{(k)}(\omega), 1 \leq k \leq Q$, are viewed as column vectors in $\mathbf{R}^{Q}$ with respect to the basis $\left\{\xi_{0}^{(k)}(\theta(t, \omega)): 1 \leq k \leq Q\right\}$. Using Cramer's rule, the above equation may be solved for each 
$u_{t}^{(k)}(\omega)$. In view of $(2.3 .13)$, this yields the following estimates

$$
\begin{aligned}
\left|u_{t}^{(k)}(\omega)\right| & =\left|\frac{\operatorname{det}\left(\eta_{t}^{(1)}(\omega), \cdots, \eta_{t}^{(k-1)}(\omega), \check{u}(\omega), \eta_{t}^{(k+1)}(\omega), \cdots, \eta_{t}^{(Q)}(\omega)\right)}{\operatorname{det}\left(\eta_{t}^{(1)}(\omega), \cdots, \eta_{t}^{(Q)}(\omega)\right)}\right| \\
& \leq \frac{|\check{u}(\omega)|}{\left|\operatorname{det}\left(\eta_{t}^{(1)}(\omega), \cdots, \eta_{t}^{(Q)}(\omega)\right)\right|} \\
& \leq \frac{\left[D_{\epsilon}(\omega)-1\right]}{Q}|u| e^{\epsilon t} \\
& \leq D_{\epsilon}(\omega)|u| e^{\epsilon t}, \quad 1 \leq k \leq Q, t \geq 0,
\end{aligned}
$$

perfectly in $\omega$. By virtue of (2.3.10), the triangle inequality and (2.3.22), one gets

$$
\left|u_{t}^{(Q+1)}(\omega)\right| \leq|u|+\sum_{k=1}^{Q}\left|u_{t}^{(k)}(\omega)\right| \leq D_{\epsilon}(\omega)|u| e^{\epsilon t}, \quad t \geq 0
$$

perfectly in $\omega$. Therefore, $T_{n}(\omega)$ satisfies (S4) perfectly in $\omega$, and the proof of the proposition is now complete.

In Lemma 2.3.4 below, an integrability condition allows us to pass from discrete-time limits of the cocycle to continuous ones. This property is crucial to the proof of Theorem 2.2.1. The reason this property holds is because the integrability hypothesis together with the perfect ergodic theorem (Lemma 2.3.1 (ii)) allow for control of the excursions of the continuous-time cocycle between discrete times.

\section{Lemma 2.3.4.}

Let $Y: \Omega \rightarrow H$ be a stationary point of the cocycle $(U, \theta)$ satisfying the integrability condition

$$
\int_{\Omega} \log ^{+} \sup _{0 \leq t_{1}, t_{2} \leq a}\left\|U\left(t_{2}, Y\left(\theta\left(t_{1}, \omega\right)\right)+(\cdot), \theta\left(t_{1}, \omega\right)\right)\right\|_{k, \epsilon} d P(\omega)<\infty
$$

for any fixed $0<\rho, a<\infty$ and $\epsilon \in(0,1]$.

Define the random field $Z: \mathbf{R}^{+} \times H \times \Omega \rightarrow H$ by

$$
Z(t, x, \omega):=U(t, x+Y(\omega), \omega)-Y(\theta(t, \omega))
$$

for $t \geq 0, x \in H, \omega \in \Omega$. Then $(Z, \theta)$ is a $C^{k, \epsilon}$ perfect cocycle. Furthermore, there is a sure event $\Omega_{5} \in \mathcal{F}$ with the following properties:

(i) $\theta(t, \cdot)\left(\Omega_{5}\right)=\Omega_{5}$ for all $t \in \mathbf{R}$,

(ii) For every $\omega \in \Omega_{5}$ and any $x \in H$, the statement

$$
\limsup _{n \rightarrow \infty} \frac{1}{n} \log |Z(n, x, \omega)|<0
$$


implies

$$
\limsup _{t \rightarrow \infty} \frac{1}{t} \log |Z(t, x, \omega)|=\limsup _{n \rightarrow \infty} \frac{1}{n} \log |Z(n, x, \omega)| .
$$

Proof.

Note that, by definition, $Z$ is a "centering" of the cocycle $U$ with respect to the stationary trajectory $\{Y(\theta(t, \cdot)): t \geq 0\}$ in the sense that $Z(t, 0, \omega)=0$ for all $(t, \omega) \in \mathbf{R}^{+} \times \Omega$. Furthermore, $(Z, \theta)$ is a $C^{k, \epsilon}$ perfect cocycle. To see this let $t_{1}, t_{2} \geq 0, \omega \in \Omega, x \in H$. Then by the perfect cocycle property for $U$, it follows that

$$
\begin{aligned}
Z\left(t_{2}, Z\left(t_{1}, x, \omega\right), \theta\left(t_{1}, \omega\right)\right) & =U\left(t_{2}, Z\left(t_{1}, x, \omega\right)+Y\left(\theta\left(t_{1}, \omega\right)\right), \theta\left(t_{1}, \omega\right)\right)-Y\left(\theta\left(t_{2}, \theta\left(t_{1}, \omega\right)\right)\right) \\
& =U\left(t_{2}, U\left(t_{1}, x+Y(\omega), \omega\right), \theta\left(t_{1}, \omega\right)\right)-Y\left(\theta\left(t_{2}+t_{1}, \omega\right)\right) \\
& =Z\left(t_{1}+t_{2}, x, \omega\right)
\end{aligned}
$$

Using the integrability condition of the lemma, the proofs of assertions (i) and (ii) follow in the same manner as for the corresponding assertions in Lemma 3.4 ([M-S.3]).

Proof of Theorem 2.2.1.

The proof of the theorem consists in two major undertakings:

(a) Using Ruelle's discrete-time analysis [Ru.2] to show that the assertions of Theorem 2.2.1 hold for the discretized cocycle, perfectly in $\omega$.

(b) Extending the discrete-time results to continuous time via perfection techniques that are essentially based on the ergodic theorem and Kingman's subadditive ergodic theorem.

Recall the auxiliary cocycle $(Z, \theta)$ defined in Lemma 2.3.4. Consider the random family of maps $F_{\omega}: \bar{B}(0,1) \rightarrow H, \omega \in \Omega$, given by $F_{\omega}(x):=Z(1, x, \omega), x \in H$, and the time-one shift $\tau:=\theta(1, \cdot): \Omega \rightarrow \Omega$. Adopting Ruelle's notation ([Ru.2], p. 272), we set $F_{\omega}^{n}:=F_{\tau^{n-1}(\omega)} \circ$ $\cdots \circ F_{\tau(\omega)} \circ F_{\omega}$. Therefore, $F_{\omega}^{n}=Z(n, \cdot, \omega)$ for each $n \geq 1$, because $(Z, \theta)$ is a cocycle. By Lemma 2.3.4, each map $F_{\omega}$ is $C^{k, \epsilon}(\epsilon \in(0,1])$ on $\bar{B}(0,1)$ and by the definition of $Z$, it follows that $\left(D F_{\omega}\right)(0)=D U(1, Y(\omega), \omega)$. By the integrability hypothesis of the theorem, it is clear that $\log ^{+}\|D U(1, Y(\cdot), \cdot)\|_{L(H)}$ is integrable. Moreover, in view of the integrability hypothesis on $(U, \theta)$, it follows that the linearized continuous-time cocycle $(D U(t, Y(\omega), \omega), \theta(t, \omega))$ and the discrete-time cocycle $\left(\left(D F_{\omega}^{n}\right)(0), \theta(n, \omega)\right)$ share the same Lyapunov spectrum, viz.:

$$
\left\{-\infty<\cdots<\lambda_{i+1}<\lambda_{i}<\cdots<\lambda_{2}<\lambda_{1}\right\}
$$

(cf. [Mo.1]). Assume that $\lambda_{i_{0}}$ is finite, that is $\lambda_{i_{0}} \in(-\infty, 0)$. Therefore, under hypotheses (I) of Theorem 5.1 in ([Ru.2], p. 272), there is a sure event $\Omega_{1}^{*} \in \mathcal{F}$ such that $\theta(t, \cdot)\left(\Omega_{1}^{*}\right)=\Omega_{1}^{*}$ for all $t \in \mathbf{R}, \overline{\mathcal{F}}$-measurable positive random variables $\rho_{1}, \beta_{1}: \Omega_{1}^{*} \rightarrow(0,1)$, and a random family of $C^{k, \epsilon}$ $(k \geq 1, \epsilon \in(0,1])$ stable submanifolds $\tilde{\mathcal{S}}_{d}(\omega)$ of $\bar{B}\left(0, \rho_{1}(\omega)\right)$ satisfying the following properties for each $\omega \in \Omega_{1}^{*}$ :

$$
\tilde{\mathcal{S}}_{d}(\omega)=\left\{x \in \bar{B}\left(0, \rho_{1}(\omega)\right):|Z(n, x, \omega)| \leq \beta_{1}(\omega) e^{\left(\lambda_{i_{0}}+\epsilon_{1}\right) n} \text { for all integers } n \geq 0\right\} .
$$


When $\lambda_{i_{0}}=-\infty$, the stable manifold is defined by

$$
\tilde{\mathcal{S}}_{d}(\omega)=\left\{x \in \bar{B}\left(0, \rho_{1}(\omega)\right):|Z(n, x, \omega)| \leq \beta_{1}(\omega) e^{\lambda n} \text { for all integers } n \geq 0\right\}
$$

where $\lambda \in(-\infty, 0)$ is arbitrary. The stable subspace $\mathcal{S}(\omega)$ of the linearized cocycle $(D U(t, Y(\omega), \omega), \theta(t, \omega))$ is tangent to the stable manifold $\tilde{\mathcal{S}}_{d}(\omega)$ at 0 ; viz. $T_{0} \tilde{\mathcal{S}}_{d}(\omega)=\mathcal{S}(\omega)$. In particular, codim $\tilde{\mathcal{S}}_{d}(\omega)$ is finite and non-random. Again by Theorem 5.1 of [Ru.2]), we have the following estimate on the Lyapunov exponent of the Lipschitz constant of $(Z(n, \cdot), \theta(n, \cdot))$ over its stable manifold:

$$
\limsup _{n \rightarrow \infty} \frac{1}{n} \log \left[\sup _{\substack{x_{1}, x_{2} \in \tilde{\mathcal{S}}_{d}(\omega) \\ x_{1} \neq x_{2}}} \frac{\left|Z\left(n, x_{1}, \omega\right)-Z\left(n, x_{2}, \omega\right)\right|}{\left|x_{1}-x_{2}\right|}\right] \leq \lambda_{i_{0}}
$$

The statements in the above paragraph hold for all $\omega$ in the $\theta(t, \cdot)$-invariant sure event $\Omega_{1}^{*}$. In order to construct such an event, we will use perfection arguments and the proof of Theorem 5.1 ([Ru.2], p. 272). Assume first that $k=1$ and $\epsilon>0$. Using the notation of [Ru.2], denote $T^{t}(\omega):=D Z(t, 0, \omega), f(\omega):=\theta(1, \omega), T_{n}(\omega):=D Z(1,0, \theta((n-1), \omega))$, for all $\omega \in \Omega$, any positive real $t$ and any integer $n \geq 1$. It is possible to replace (5.3) in [Ru.2], p. 274) by its continuous-time perfect analogue

$$
\lim _{t \rightarrow \infty} \frac{1}{t} \log ^{+}\|Z(1, \cdot, \theta(t, \omega))\|_{1, \epsilon}=0 .
$$

This follows from the integrability hypothesis of the theorem and the perfect ergodic theorem (Lemma 2.3.1 (ii)). More specifically, (2.3.27) holds for all $\omega$ in a sure event $\Omega_{1}^{*} \in \mathcal{F}$ such that $\theta(t, \cdot)\left(\Omega_{1}^{*}\right)=\Omega_{1}^{*}$ for all $t \in \mathbf{R}$. Assume $\lambda_{i_{0}}>-\infty$. Adopting the terminology of Theorem 1.1 ([Ru.2], p. 248), take $S=i_{0}-1$, and $\mu^{(S+1)}=\lambda_{i_{0}}$. In case $\lambda_{i_{0}}=-\infty$, take $\mu^{(S+1)}$ to be any fixed number in $(-\infty, 0)$. The integrability hypothesis on $U$ coupled with Lemma 2.3.3 (where $j_{0}=i_{0}-1$ ) imply the existence of a sure event $\Omega_{2}^{*} \in \mathcal{F}$ such that $\Omega_{2}^{*} \subseteq \Omega_{1}^{*}, \theta(t, \cdot)\left(\Omega_{2}^{*}\right)=\Omega_{2}^{*}$ for all $t \in \mathbf{R}$, and the sequence $T_{n}(\omega), V_{n}(\omega):=E_{i_{0}}\left(\tau^{n}(\omega)\right), n \geq 1$, satisfies Conditions (S) of [Ru.2], p. 256) for every $\omega \in \Omega_{2}^{*}$. Pick and fix any $\omega \in \Omega_{2}^{*}$. As in the proof of Theorem 5.1 ([Ru.2], pp. 274-278), the "perturbation theorem" (Theorem 4.1, [Ru.2], pp. 262-263) holds for the sequence $T_{n}(\omega), n \geq 1$. Thus the assertions in the previous paragraph are valid for $k=1$ and any $\epsilon \in(0,1]$. When $k>1$ and $\epsilon \in(0,1])$, we first apply the previous analysis to the perfect cocycle

$$
\left(\check{Z}\left(t, x, x_{1}, \omega\right):=\left(Z(t, x, \omega), D Z(t, x, \omega) x_{1}\right), \theta(t, \omega)\right), \quad x, x_{1} \in H, t \geq 0,
$$

on $H \oplus H$. Secondly, we use the inductive argument of ([Ru.2], pp. 278-279) to show that the $\tilde{\mathcal{S}}_{d}(\omega)$ are $C^{k, \epsilon}$ manifolds $(k>1, \epsilon \in(0,1])$ perfectly in $\omega$.

To establish assertion (a) of the theorem, let $\tilde{\mathcal{S}}(\omega), \omega \in \Omega_{1}^{*}$, be the set defined therein. Then the definition of $Z$ and property (2.3.25) of $\tilde{\mathcal{S}}_{d}(\omega)$ imply that

$$
\tilde{\mathcal{S}}(\omega)=\tilde{\mathcal{S}}_{d}(\omega)+Y(\omega)
$$


for all $\omega \in \Omega_{1}^{*}$. Thus $\tilde{\mathcal{S}}(\omega)$ is a $C^{k, \epsilon}$ manifold $(k>1, \epsilon \in(0,1])$, with tangent space $T_{Y(\omega)} \tilde{\mathcal{S}}(\omega)=$ $T_{0} \tilde{\mathcal{S}}_{d}(\omega)=\mathcal{S}(\omega)$. In particular, codim $\tilde{\mathcal{S}}(\omega)=\operatorname{codim} \mathcal{S}(\omega), \omega \in \Omega_{1}^{*}$, is finite and non-random.

To complete the proof of the inequality (2.2.1) in part (a) of the theorem, use (2.3.26) to get

$$
\limsup _{n \rightarrow \infty} \frac{1}{n} \log |Z(n, x, \omega)| \leq \lambda_{i_{0}}
$$

perfectly in $\omega$ for all $x \in \tilde{\mathcal{S}}_{d}(\omega)$. In view of Lemma 2.3.4, we may extend the above estimate to cover its continuous-time counterpart. Hence we obtain a sure event $\Omega_{3}^{*} \subseteq \Omega_{2}^{*}, \Omega_{3}^{*} \in \mathcal{F}$, such that $\theta(t, \cdot)\left(\Omega_{3}^{*}\right)=\Omega_{3}^{*}$ for all $t \in \mathbf{R}$, and

$$
\limsup _{t \rightarrow \infty} \frac{1}{t} \log |Z(t, x, \omega)| \leq \lambda_{i_{0}}
$$

for all $\omega \in \Omega_{3}^{*}$ and all $x \in \tilde{\mathcal{S}}_{d}(\omega)$. The above inequality together with definition of $Z$ imply the estimate (2.2.1) of the theorem.

Next, we establish assertion (b) of the theorem. To do so, let $\omega \in \Omega_{1}^{*}$ and $x \in \tilde{\mathcal{S}}_{d}(\omega)$. Then by (2.3.26), it follows that there is a positive integer $N_{0}:=N_{0}(\omega)$, independent of $x \in \tilde{\mathcal{S}}_{d}(\omega)$, such that $Z(n, x, \omega) \in \bar{B}(0,1)$ for all $n \geq N_{0}$. Now Lemma 2.3.1(ii) gives a $\theta(t, \cdot)$-invariant sure event $\Omega_{3}$ such that

$$
\lim _{t \rightarrow \infty} \frac{1}{t} \log ^{+} \sup _{\substack{0 \leq u \leq 1,\left(v^{*}, \eta^{*}\right) \in \bar{B}(0,1)}}\left\|D Z\left(u,\left(v^{*}, \eta^{*}\right), \theta(t, \omega)\right)\right\|_{L(H)}=0
$$

for all $\omega \in \Omega_{3}$. Define the sure event $\Omega_{4}^{*}:=\Omega_{3}^{*} \cap \Omega_{3} \in \mathcal{F}$. Clearly, $\theta(t, \cdot)\left(\Omega_{4}^{*}\right)=\Omega_{4}^{*}$ for all $t \in \mathbf{R}$. By the definition of $Z$ and the Mean Value Theorem, we obtain the following inequalities

$$
\begin{aligned}
\sup _{n \leq t \leq n+1} \frac{1}{t} \log \left[\sup _{\substack{x_{1} \neq x_{2},\left(v_{1}, \eta_{1}\right), x_{2} \in \tilde{\mathcal{S}}(\omega)}} \frac{\left|U\left(t, x_{1}, \omega\right)-U\left(t, x_{2}, \omega\right)\right|}{\left|x_{1}-x_{2}\right|}\right] \\
=\sup _{n \leq t \leq n+1} \frac{1}{t} \log \left[\sup _{\substack{x_{1} \neq x_{2}, x_{1}, x_{2} \in \mathcal{S}_{d}(\omega)}} \frac{\left|Z\left(t, x_{1}, \omega\right)-Z\left(t, x_{2}, \omega\right)\right|}{\left|x_{1}-x_{2}\right|}\right] \\
\leq \frac{1}{n} \log ^{+} \sup _{\substack{0 \leq u \leq 1,\left(v^{*}, \eta^{*}\right) \in \bar{B}(0,1)}}\left\|D Z\left(u,\left(v^{*}, \eta^{*}\right), \theta(n, \omega)\right)\right\|_{L(H)} \\
\quad+\frac{n}{(n+1)} \frac{1}{n} \log \left[\sup _{\substack{x_{1} \neq x_{2}, x_{1}, x_{2} \in \mathcal{\mathcal { S }}_{d}(\omega)}} \frac{\left|Z\left(n, x_{1}, \omega\right)-Z\left(n, x_{2}, \omega\right)\right|}{\left|x_{1}-x_{2}\right|}\right.
\end{aligned}
$$

for all $\omega \in \Omega_{4}^{*}$, and all sufficiently large $n \geq N_{0}(\omega)$. Now take $\limsup _{n \rightarrow \infty}$ on both sides of the above inequality, and use (2.3.26), (2.3.29') in order to complete the proof assertion (b) of the theorem.

The cocycle invariance (2.2.3) in part (c) of the theorem follows immediately from the Oseledec-Ruelle theorem (Theorem 2.1.1) applied to the perfect linearized cocycle $(D U(t, Y(\omega), \omega), \theta(t, \omega))$. Indeed, one gets a sure $\theta(t, \cdot)$-invariant event $\in \mathcal{F}$ (also denoted by $\Omega_{1}^{*}$ ), such that $D U(t, Y(\omega), \omega)(\mathcal{S}(\omega)) \subseteq \mathcal{S}(\theta(t, \omega))$ for all $t \geq 0$ and all $\omega \in \Omega_{1}^{*}$. 
The proof of the asymptotic invariance property (2.2.2) of the non-linear cocycle requires some work. To achieve this, we will extend the arguments underlying the proofs of Theorems 5.1 and 4.1 in [Ru.2], pp. 262-279, to a continuous time setting. The crucial step towards this goal is to show that the two random variables $\rho_{1}, \beta_{1}$ in (2.3.25) may be redefined on a sure event (also denoted by) $\Omega_{1}^{*}$ such that $\theta(t, \cdot)\left(\Omega_{1}^{*}\right)=\Omega_{1}^{*}$ for all $t \in \mathbf{R}$, and

$$
\rho_{1}(\theta(t, \omega)) \geq \rho_{1}(\omega) e^{\left(\lambda_{i_{0}}+\epsilon_{1}\right) t}, \quad \beta_{1}(\theta(t, \omega)) \geq \beta_{1}(\omega) e^{\left(\lambda_{i_{0}}+\epsilon_{1}\right) t}
$$

for every $\omega \in \Omega_{1}^{*}$ and all $t \geq 0$. For the given choice of $\epsilon_{1}$, fix $0<\epsilon_{3}<-\epsilon\left(\lambda_{i_{0}}+\epsilon_{1}\right) / 4$, where $\epsilon \in(0,1]$ denotes the Hölder exponent of $U$. The above inequalities hold in the discrete case (when $t=n$, a positive integer) because of Theorem 5.1 (c) ([Ru.2], p. 274). To prove them for any continuous time $t$, we will modify the definitions of $\rho_{1}, \beta_{1}$ in the proofs of Theorems 5.1 and 4.1 in [Ru.2]. In the notation of the proof of Theorem 5.1 ([Ru.2], p. 274), we replace the random variable $G$ in (5.4) ([Ru.2], p. 274) by the larger one

$$
\tilde{G}(\omega):=\sup _{t \geq 0}\|Z(1, \cdot, \theta(t, \omega))\|_{1, \epsilon} e^{\left(-t \epsilon_{3}-\lambda \epsilon\right)} .
$$

Clearly, $\tilde{G}(\omega)$ is finite perfectly in $\omega$, because of (2.3.27) and Lemma 2.3.2. Following ([Ru.2], pp. $266,274)$, the random variables $\rho_{1}, \beta_{1}$ may be chosen according to the relations

$$
\begin{aligned}
& \beta_{1}:=\left[\frac{\delta_{1} \wedge\left(\frac{1}{\sqrt{2} A}\right)}{2 \tilde{G}}\right]^{\frac{1}{\epsilon}} \wedge 1 \\
& \rho_{1}:=\frac{\beta_{1}}{B_{\epsilon_{3}}}
\end{aligned}
$$

where $A, \delta_{1}$ and $B_{\epsilon_{3}}$ are random positive constants that are defined via continuous-time analogues of the relations (4.26), (4.18)-(4.21), (4.24), (4.25) in [Ru.2], pp. 265-267, with $\eta$ replaced by $\epsilon_{3}$. In particular, the "ancestry" of $A, \delta_{1}$ and $B_{\epsilon_{3}}$ in Ruelle's argument may be traced back to the constants $D_{\epsilon_{3}}, K_{\epsilon_{3}}$ which appear in Lemmas 2.3.3 and 2.3.2 of this article. Hence (2.3.30) will follow if we can show that, for sufficiently small $\epsilon_{3}>0$, the following inequalities

$$
\left.\begin{array}{c}
K_{\epsilon_{3}}(\theta(l, \omega)) \leq K_{\epsilon_{3}}(\omega)+\frac{\epsilon_{3} l}{2} \\
D_{\epsilon_{3}}(\theta(l, \omega)) \leq e^{\frac{\epsilon_{3} l}{2}} D_{\epsilon_{3}}(\omega) \\
\tilde{G}(\theta(l, \omega)) \leq e^{\epsilon_{3} l} \tilde{G}(\omega)
\end{array}\right\}
$$

hold perfectly in $\omega$ for all real $l \geq 0$. The first and second inequalities in (2.3.34) follow from Lemmas 2.3.2(ii) and 2.3.3, respectively. The third inequality is an immediate consequence of the definition of $\tilde{G}$ in (2.3.31). The proof of (2.3.30) is now complete in view of (2.3.32), (2.3.33) and (2.3.34).

The inequalities in (2.3.30) will allow us to establish the asymptotic invariance property (2.2.2) in (c) of the theorem. By the perfect inequality in (b), there is a sure event $\Omega_{5}^{*} \subseteq \Omega_{4}^{*}$ such 
that $\theta(t, \cdot)\left(\Omega_{5}^{*}\right)=\Omega_{5}^{*}$ for all $t \in \mathbf{R}$, and for any $0<\epsilon^{\prime}<\epsilon_{1}$ and any $\omega \in \Omega_{5}^{*}$, there exists $\beta^{\epsilon^{\prime}}(\omega)>0$ (independent of $x$ ) so that

$$
|U(t, x, \omega)-Y(\theta(t, \omega))| \leq \beta^{\epsilon^{\prime}}(\omega) e^{\left(\lambda_{i_{0}}+\epsilon^{\prime}\right) t}
$$

for all $x \in \tilde{\mathcal{S}}(\omega), t \geq 0$. Let $t$ be any positive real, $n$ a non-negative integer, $\omega \in \Omega_{5}^{*}$ and $x \in \tilde{\mathcal{S}}(\omega)$. Using the cocycle property and (2.3.35), we obtain

$$
\begin{aligned}
|U(n, U(t, x, \omega), \theta(t, \omega))-Y(\theta(n, \theta(t, \omega)))| & =|U(n+t, x, \omega)-Y(\theta(n+t, \omega))| \\
& \leq \beta^{\epsilon^{\prime}}(\omega) e^{\left(\lambda_{i_{0}}+\epsilon^{\prime}\right)(n+t)} \\
& \leq \beta^{\epsilon^{\prime}}(\omega) e^{\left(\lambda_{i_{0}}+\epsilon^{\prime}\right) t} e^{\left(\lambda_{i_{0}}+\epsilon_{1}\right) n} .
\end{aligned}
$$

Using (2.3.30), (2.3.35), (2.3.36) and the definition of $\tilde{\mathcal{S}}(\theta(t, \omega))$, we see that for each $\omega \in \Omega_{5}^{*}$, there exists $\tau_{1}(\omega)>0$ such that $U(t, x, \omega) \in \tilde{\mathcal{S}}(\theta(t, \omega))$ for all $t \geq \tau_{1}(\omega)$. Hence, for all $\omega \in \Omega_{5}^{*}$,

$$
U(t, \cdot, \omega)(\tilde{\mathcal{S}}(\omega)) \subseteq \tilde{\mathcal{S}}(\theta(t, \omega)), \quad t \geq \tau_{1}(\omega)
$$

and the proof of assertion (c) is complete.

Our next objective is to establish the existence of the perfect family of local unstable manifolds $\tilde{\mathcal{U}}(\omega)$ in assertion (d) of the theorem. To this end, we define the random field $\hat{Z}: \mathbf{R}^{+} \times H \times \Omega \rightarrow$ $H$ by

$$
\hat{Z}(t, x, \omega):=U(t, x+Y(\theta(-t, \omega)), \theta(-t, \omega))-Y(\omega)
$$

for all $t \geq 0, x \in H, \omega \in \Omega$. Note that $\hat{Z}(t, \cdot, \omega)=Z(t, \cdot, \theta(-t, \omega)), t \geq 0, \omega \in \Omega$; and $\hat{Z}$ is $\left(\mathcal{B}\left(\mathbf{R}^{+}\right) \otimes\right.$ $\mathcal{B}(H) \otimes \mathcal{F}, \mathcal{B}(H))$-measurable. Since $Y$ is a stationary point for $(U, \theta)$, we may replace $\omega$ by $\theta(-t, \omega)$ in (2.1.1). Thus $\hat{Z}(t, 0, \omega)=0$ for all $t \geq 0, \omega \in \Omega$. We contend that $\left([D \hat{Z}(t, 0, \omega)]^{*}, \theta(-t, \omega), t \geq 0\right)$ is a perfect linear cocycle in $L(H)$. To see this, we first observe that $(D U(t, Y(\omega), \omega), \theta(t, \omega))$ is an $L(H)$-valued perfect cocycle:

$$
D U\left(t_{1}+t_{2}, Y(\omega), \omega\right)=D U\left(t_{1}, Y\left(\theta\left(t_{2}, \omega\right)\right), \theta\left(t_{2}, \omega\right)\right) \circ D U\left(t_{2}, Y(\omega), \omega\right)
$$

for all $\omega \in \Omega, t_{1}, t_{2} \geq 0$. Secondly, we replace $\omega$ by $\theta\left(-t_{1}-t_{2}, \omega\right)$ and take adjoints in the above identity to obtain

$$
\begin{aligned}
& {\left[D U\left(t_{1}+t_{2}, Y\left(\theta\left(-t_{1}-t_{2}, \omega\right)\right), \theta\left(-t_{1}-t_{2}, \omega\right)\right)\right]^{*}} \\
& \quad=\left[D U\left(t_{2}, Y\left(\theta\left(-t_{1}-t_{2}, \omega\right)\right), \theta\left(-t_{1}-t_{2}, \omega\right)\right)\right]^{*} \circ\left[D U\left(t_{1}, Y\left(\theta\left(-t_{1}, \omega\right)\right), \theta\left(-t_{1}, \omega\right)\right]^{*}\right.
\end{aligned}
$$

for all $\omega \in \Omega, t_{1}, t_{2} \geq 0$. Therefore,

$$
\left[D \hat{Z}\left(t_{1}+t_{2}, 0, \omega\right)\right]^{*}=\left[D \hat{Z}\left(t_{2}, 0, \theta\left(-t_{1}, \omega\right)\right)\right]^{*} \circ\left[D \hat{Z}\left(t_{1}, 0, \omega\right)\right]^{*}
$$

for all $\omega \in \Omega, t_{1}, t_{2} \geq 0$; and our contention is proved. 
We will now apply the Oseledec-Ruelle theorem to the perfect cocycle $\left([D \hat{Z}(t, 0, \omega)]^{*}\right.$, $\theta(-t, \omega), t \geq 0)$. To do this, it is sufficient to check the integrability condition

$$
\int_{\Omega} \log ^{+} \sup _{0 \leq t_{1}, t_{2} \leq a}\left\|\left[D \hat{Z}\left(t_{2}, 0, \theta\left(-t_{1}, \omega\right)\right)\right]^{*}\right\|_{L(H)} d P(\omega)<\infty
$$

for any fixed $a \in(0, \infty)$. The above integrability relation follows from the integrability hypothesis of Theorem 2.2.1 and the $P$-preserving property of $\theta(t, \cdot)$ :

$$
\begin{aligned}
& \int_{\Omega} \log ^{+} \sup _{0 \leq t_{1}, t_{2} \leq a}\left\|\left[D \hat{Z}\left(t_{2}, 0, \theta\left(-t_{1}, \omega\right)\right)\right]^{*}\right\|_{L(H)} d P(\omega) \\
& \left.\quad=\int_{\Omega} \log ^{+} \sup _{0 \leq t_{1}, t_{2} \leq a} \| D U\left(t_{2}, Y\left(\theta\left(-t_{2}-t_{1}, \omega\right)\right), \theta\left(-t_{2}-t_{1}, \omega\right)\right)\right) \|_{L(H)} d P(\omega) \\
& \left.\quad \leq \int_{\Omega} \log ^{+} \sup _{0 \leq t_{1} \leq 2 a, 0 \leq t_{2} \leq a} \| D U\left(t_{2}, Y\left(\theta\left(t_{1}, \omega\right)\right), \theta\left(t_{1}, \omega\right)\right)\right) \|_{L(H)} d P(\omega) \\
& \left.\quad \leq \int_{\Omega} \log ^{+} \sup _{0 \leq t_{1} \leq a, 0 \leq t_{2} \leq a} \| D U\left(t_{2}, Y\left(\theta\left(t_{1}, \omega\right)\right), \theta\left(t_{1}, \omega\right)\right)\right) \|_{L(H)} d P(\omega) \\
& \left.\quad+\int_{\Omega} \log ^{+} \sup _{a \leq t_{1} \leq 2 a, 0 \leq t_{2} \leq a} \| D U\left(t_{2}, Y\left(\theta\left(t_{1}-a, \omega\right)\right), \theta\left(t_{1}-a, \omega\right)\right)\right) \|_{L(H)} d P(\omega) \\
& \left.\quad=2 \int_{\Omega} \log ^{+} \sup _{0 \leq t_{1}, t_{2} \leq a} \| D U\left(t_{2}, Y\left(\theta\left(t_{1}, \omega\right)\right), \theta\left(t_{1}, \omega\right)\right)\right) \|_{L(H)} d P(\omega)<\infty .
\end{aligned}
$$

By (2.3.38) and the Oseledec-Ruelle theorem, we conclude that the linear cocycle $\left([D \hat{Z}(t, 0, \omega)]^{*}, \theta(-t, \omega), t \geq 0\right)$ has a fixed discrete Lyapunov spectrum. Furthermore, this spectrum (with multiplicities) coincides with that of the cocycle $(D U(t, Y(\omega), \omega), \theta(t, \omega))$, viz. $\left\{\cdots \lambda_{i+1}<\right.$ $\left.\lambda_{i}<\cdots<\lambda_{2}<\lambda_{1}\right\}$ where $\lambda_{i} \neq 0$ for all $i \geq 1$, by hyperbolicity. See [Ru.2], Section 3.5, p. 261.

The next step in our construction of the perfect random family of local unstable manifolds $\tilde{\mathcal{U}}(\omega)$ starts with the following estimate:

$$
\int_{\Omega} \log ^{+} \sup _{0 \leq t_{1}, t_{2} \leq 1}\left\|\hat{Z}\left(t_{2}, \cdot, \theta\left(-t_{1}, \omega\right)\right)\right\|_{k, \epsilon} d P(\omega)<\infty .
$$

By the same argument as in the previous paragraph, the above estimate is a consequence of the $P$ preserving property of $\theta(t, \cdot), t \in \mathbf{R}$, and the integrability hypothesis of the theorem. Define $\lambda_{i_{0}-1}$ as in the statement of Theorem 2.2.1, and fix any $\epsilon_{2} \in\left(0, \lambda_{i_{0}-1}\right)$. In view of the above integrability property, it follows from Lemma 2.3 .3 that the sequence $\tilde{T}_{n}(\omega):=[D \hat{Z}(1,0, \theta(-n, \omega))]^{*}, \theta(-n, \omega)$, $n \geq 0$, satisfies Condition (S) of [Ru.2] perfectly in $\omega$. Hence the sequence $\tilde{T}_{n}(\omega), n \geq 1$, satisfies Corollary 3.4 ([Ru.2], p. 260) perfectly in $\omega$, because of Proposition 3.3 in [Ru.2]. At this point, we may modify the arguments in the proof of Ruelle's Theorem 6.1 ([Ru.2], p. 280) using an approach analogous to the one used in constructing the stable manifolds in this proof. Therefore, one gets a $\theta(-t, \cdot)$-invariant sure event $\hat{\Omega}_{1}^{*} \in \mathcal{F}$ and $\overline{\mathcal{F}}$-measurable random variables $\rho_{2}, \beta_{2}: \hat{\Omega}_{1}^{*} \rightarrow(0,1)$ satisfying the following properties. If $\lambda_{i_{0}-1}<\infty$, define $\tilde{\mathcal{U}}_{d}(\omega)$ to be the set of all $x_{0} \in \bar{B}\left(0, \rho_{2}(\omega)\right)$ with the property that there is a discrete "history" process $u(-n, \cdot): \Omega \rightarrow H, n \geq 0$, such that $u(0, \omega)=x_{0}, \hat{Z}(1, u(-(n+1), \omega), \theta(-n, \omega))=u(-n, \omega)$ and $|u(-n, \omega)| \leq \beta_{2}(\omega) e^{-n\left(\lambda_{i_{0}-1}-\epsilon_{2}\right)}$ for 
all $n \geq 0$. If $\lambda_{i_{0}-1}=\infty$, define $\tilde{\mathcal{U}}_{d}(\omega)$ to be the set of all $x_{0} \in H$ with the property that there is a discrete history process $u(-n, \cdot): \Omega \rightarrow H, n \geq 0$, such that $u(0, \omega)=x_{0}$, and $|u(-n, \omega)| \leq$ $\beta_{2}(\omega) e^{-\lambda n}$ for all $n \geq 0$ and arbitrary $\lambda>0$. It follows from ([Ru.2], p. 281) that the discrete history process $u(-n, \cdot)$ is uniquely determined by $x_{0}$. Moreover, each $\tilde{\mathcal{U}}_{d}(\omega), \omega \in \hat{\Omega}_{1}^{*}$, is a $C^{k, \epsilon}$ $(k \geq 1, \epsilon \in(0,1])$ finite-dimensional submanifold of $\bar{B}\left(0, \rho_{2}(\omega)\right)$ with tangent space $\mathcal{U}(\omega)$ at 0 , and $\operatorname{dim} \tilde{\mathcal{U}}_{d}(\omega)$ is fixed independently of $\omega$ and $\epsilon_{2}$. Furthermore,

$$
\rho_{2}(\theta(-t, \omega)) \geq \rho_{2}(\omega) e^{-\left(\lambda_{i_{0}}-1-\epsilon_{2}\right) t}, \quad \beta_{2}(\theta(-t, \omega)) \geq \beta_{2}(\omega) e^{-\left(\lambda_{i_{0}}-1-\epsilon_{2}\right) t} .
$$

perfectly in $\omega$ for all $t \geq 0$. We claim that the set $\tilde{\mathcal{U}}(\omega)$ defined in (d) of Theorem 2.2.1 coincides with $\tilde{\mathcal{U}}_{d}(\omega)+Y(\omega)$ for each $\omega \in \hat{\Omega}_{1}^{*}$. We first show that $\tilde{\mathcal{U}}_{d}(\omega)+Y(\omega) \subseteq \tilde{\mathcal{U}}(\omega)$. Let $x_{0} \in \tilde{\mathcal{U}}_{d}(\omega)$ and $u$ be as above. Set

$$
y_{0}(-n, \omega):=u(-n, \omega)+Y(\theta(-n, \omega)), \quad n \geq 0 .
$$

It is easy to check that $y_{0}$ is a discrete history process satisfying the first and second assertions in (d) of the theorem. Hence $x_{0}+Y(\omega) \in \tilde{\mathcal{U}}(\omega)$. Similarly, $\tilde{\mathcal{U}}(\omega) \subseteq \tilde{\mathcal{U}}_{d}(\omega)+Y(\omega)$ for all $\omega \in \hat{\Omega}_{1}^{*}$. Hence $\tilde{\mathcal{U}}(\omega)=\tilde{\mathcal{U}}_{d}(\omega)+Y(\omega)$ for all $\omega \in \hat{\Omega}_{1}^{*}$. This immediately implies that $\tilde{\mathcal{U}}(\omega)$ is a $C^{k, \epsilon}(k \geq 1, \epsilon \in(0,1])$ finite-dimensional submanifold of $\bar{B}\left(Y(\omega), \rho_{2}(\omega)\right)$, and

$$
T_{Y(\omega)} \tilde{\mathcal{U}}(\omega)=T_{0} \tilde{\mathcal{U}}_{d}(\omega)=\mathcal{U}(\omega)
$$

for all $\omega \in \hat{\Omega}_{1}^{*}$.

We will next address the issue of the existence of the continuous-time history process satisfying the third assertion in part (d) of the theorem. Suppose $x \in \tilde{\mathcal{U}}(\omega)$. From what we proved in the previous paragraph, it follows that there is an $x_{0} \in \mathcal{U}_{d}(\omega)$ such that $x=x_{0}+Y(\omega)$. The discrete process $y_{0}$ given by (2.3.40) may be extended to a continuous-time history process $y(\cdot, \omega):(-\infty, 0] \rightarrow H$ such that $y(0, \omega)=x$, and $y(\cdot, \omega)$ satisfies the third assertion in $(\mathrm{d})$. This is achieved by interpolation within the periods $[-(n+1),-n], n \geq 0$, using the cocycle property of $U$ : Indeed, let $s \in(-(n+1),-n)$. Then there is an $\alpha \in(0,1)$, such that $s=\alpha-(n+1)$. Define

$$
y(s, \omega):=U\left(s+n+1, y_{0}(-(n+1), \omega), \theta(-(n+1), \omega)\right) .
$$

Obviously, $y(0, \omega)=x_{0}+Y(\omega)=x$. Let $s \in(-(n+1),-n)$ and suppose $0<t \leq-s$. Pick a positive integer $m<n$ such that $s+t \in[-(m+1),-m]$. The above definition of $y$, together with the perfect cocycle property for $U$, easily imply that

$$
y(t+s, \omega)=U(t, y(s, \omega), \theta(s, \omega))
$$

In particular, $U(t, y(-t, \omega), \theta(-t, \omega))=x$ for all $t \geq 0$. This follows from (2.3.41) when $s$ is replaced by $-t$. Furthermore, for each $x \in \tilde{\mathcal{U}}(\omega)$, the above continuous-time history process is uniquely determined because its discrete-time counterpart is unique. 
We will now prove the following estimate

$$
\limsup _{t \rightarrow \infty} \frac{1}{t} \log |y(-t, \omega)-Y(\theta(-t, \omega))| \leq-\lambda_{i_{0}-1}
$$

perfectly in $\omega$. We start with its discrete-time counterpart

$$
\limsup _{n \rightarrow \infty} \frac{1}{n} \log |y(-n, \omega)-Y(\theta(-n, \omega))| \leq-\lambda_{i_{0}-1}
$$

which holds perfectly in $\omega$, because of Theorem 6.1 (b) in [Ru.2]. Let $t \in(n, n+1)$. Then there exists $\gamma \in(0,1)$ such that $-t=\gamma-(n+1)$. Thus by the definition of $y$ and the Mean Value Theorem, it follows that

$$
\begin{aligned}
& |y(-t, \omega)-Y(\theta(-t, \omega))| \\
& =\mid U(\gamma, y(-(n+1), \omega), \theta(-(n+1), \omega))-U(\gamma, Y(\theta(-(n+1), \omega), \theta(-(n+1), \omega)) \mid \\
& \leq \sup _{\substack{\left(v^{*}, \eta^{*}\right) \in \bar{B}(0,1), \gamma \in(0,1)}}\left\|D U\left(\gamma,\left(v^{*}, \eta^{*}\right)+Y(\theta(-(n+1), \omega)), \theta(-(n+1), \omega)\right)\right\|_{L(H)} \\
& \quad \times \mid y(-(n+1), \omega)-Y(\theta(-(n+1), \omega))) \mid
\end{aligned}
$$

perfectly in $\omega$. Letting $t \rightarrow \infty$, we get

$$
\begin{aligned}
& \limsup _{t \rightarrow \infty} \frac{1}{t} \log |y(-t, \omega)-Y(\theta(-t, \omega))| \\
& \leq \limsup _{n \rightarrow \infty} \frac{1}{n} \log ^{+} \sup _{\substack{\left(v^{*}, \eta^{*}\right) \in \bar{B}(0,1), \gamma \in(0,1)}}\left\|D U\left(\gamma,\left(v^{*}, \eta^{*}\right)+Y(\theta(-(n+1), \omega)), \theta(-(n+1), \omega)\right)\right\|_{L(H)} \\
& \left.\quad+\limsup _{n \rightarrow \infty} \frac{1}{n} \log \mid y(-(n+1), \omega)-Y(\theta(-(n+1), \omega))\right) \mid .
\end{aligned}
$$

By the integrability condition of the theorem and the perfect ergodic theorem (Lemma 2.3.1 (ii)), the first term on the right hand side of the above inequality is zero, perfectly in $\omega \in \Omega$. Since $y(0) \in \tilde{\mathcal{U}}(\omega)$, the second term is less than or equal to $-\lambda_{i_{0}-1}$. This completes the proof of assertion (d) of the theorem.

We will omit the proof of assertion (e), since it is very similar to that of (2.3.42).

Our next objective is to prove assertion (f) of the theorem. Note first that the perfect invariance

$$
D U(t, \cdot, \theta(-t, \omega))(\mathcal{U}(\theta(-t, \omega)))=\mathcal{U}(\omega), \quad t \geq 0,
$$

follows from the cocycle property for the linearized semiflow and Theorem 2.1.2); cf. [Mo.1], Corollary $2(\mathrm{v})$ of Theorem 4 . Since $\operatorname{dim} \mathcal{U}(\omega)$ is fixed and finite perfectly in $\omega$, the restriction

$$
D U(t, \cdot, \theta(-t, \omega)) \mid \mathcal{U}(\theta(-t, \omega)): \mathcal{U}(\theta(-t, \omega)) \rightarrow \mathcal{U}(\omega), \quad t \geq 0
$$

is a linear homeomorphism onto. It remains to check the following asymptotic invariance property in $(f)$ :

$$
\tilde{\mathcal{U}}(\omega) \subseteq U(t, \cdot, \theta(-t, \omega))(\tilde{\mathcal{U}}(\theta(-t, \omega))), \quad t \geq \tau_{2}(\omega)
$$


perfectly in $\omega$ for some $\tau_{2}(\omega)>0$. Suppose $x \in \tilde{\mathcal{U}}(\omega)$. Then by assertions (d), (e) of the theorem and inequalities (2.3.39), there exist a (unique) history process $y(-t, \omega), t \geq 0$, and a random time $\tau_{2}(\omega)>0$ satisfying the following: $y(0, \omega)=x, y(-t, \omega) \in \bar{B}\left(Y(\theta(-t, \omega)), \rho_{2}(\theta(-t, \omega))\right)$ for all $t \geq \tau_{2}(\omega)$, and

$$
y\left(t^{\prime}-t, \omega\right)=U\left(t^{\prime}, y(-t, \omega), \theta(-t, \omega)\right), \quad 0<t^{\prime} \leq t,
$$

perfectly in $\omega$. Pick any $t_{1} \geq \tau_{2}(\omega)$. Then $x=U\left(t_{1}, y\left(-t_{1}, \omega\right), \theta\left(-t_{1}, \omega\right)\right.$ ), because of $(2.3 .45)$ (for $\left.t=t^{\prime}=t_{1}\right)$. Now $\left.y\left(-t_{1}, \omega\right) \in \tilde{\mathcal{U}}\left(\theta\left(-t_{1}, \omega\right)\right)\right)$. To prove this, we define the process $y_{1}(-t, \omega):=$ $y\left(-t-t_{1}, \omega\right), t \geq 0$. Hence $y_{1}(\cdot, \omega)$ is a history process and

$$
y_{1}(0, \omega)=y\left(-t_{1}, \omega\right) \in \bar{B}\left(Y\left(\theta\left(-t_{1}, \omega\right)\right), \rho_{2}\left(\theta\left(-t_{1}, \omega\right)\right)\right) .
$$

Therefore $\left.y\left(-t_{1}, \omega\right) \in \tilde{\mathcal{U}}\left(\theta\left(-t_{1}, \omega\right)\right)\right)$. This implies (2.3.44) because $t_{1} \geq \tau_{2}(\omega)$ is arbitrary.

To prove the transversality property in in $(\mathrm{g})$, note the following perfect identities:

$$
T_{Y(\omega)} \tilde{\mathcal{U}}(\omega)=\mathcal{U}(\omega), \quad T_{Y(\omega)} \tilde{\mathcal{S}}(\omega)=\mathcal{S}(\omega), \quad H=\mathcal{U}(\omega) \oplus \mathcal{S}(\omega)
$$

All the assertions (a)-(g) of the theorem will hold perfectly in $\omega$ if we take $\Omega^{*}:=\Omega_{1}^{*} \cap \hat{\Omega}_{1}^{*}$.

To deal with the case when $U$ is a $C^{\infty}$ cocycle, we adapt the proof in [Ru.2], section (5.3) (p. 297). Thus we obtain a $\theta(t, \cdot)$-invariant sure event in $\mathcal{F}$ (also denoted by $\Omega^{*}$ ) such that $\tilde{\mathcal{S}}(\omega)$ and $\tilde{\mathcal{U}}(\omega)$ are $C^{\infty}$ for all $\omega \in \Omega^{*}$. This completes the proof of Theorem 2.2.1.

\subsection{The local stable manifold theorem for see's and spde's.}

In this section, we discuss several classes of semilinear stochastic evolutions equations and spde's. The objective is to establish sufficient conditions for a local stable manifold theorem for each class.

(a) Stochastic semilinear evolution equations: Additive noise.

Let $K, H$ be two separable real Hilbert spaces. Let $A$ be a self-adjoint operator on $H$ such that $A \geq c I_{H}$, where $c$ is a real constant and $I_{H}$ is the identity operator on $H$. Assume that $A$ admits a discrete non-vanishing spectrum $\left\{\mu_{n}, n \geq 1\right\}$ which is bounded below. Let $\left\{e_{n}, n \geq 1\right\}$ denote a basis for $H$ consisting of eigen vectors of $A$, viz. $A e_{n}=\mu_{n} e_{n}, n \geq 1$. Assume further that $A^{-1}$ is trace-class. Suppose $B_{0} \in L_{2}(K, H)$. Let $W(t), t \in \mathbf{R}$, be a Brownian motion on the canonical filtered Wiener space $\left(\Omega, \mathcal{F},\left(\mathcal{F}_{t}\right)_{t \geq 0}, P\right)$ and with a separable covariance Hilbert space $K$ (Section 1.2). Let $T_{t}=e^{-A t}$ stand for the strongly continuous semigroup generated by $-A$.

Denote by $\mu_{m}$ the largest negative eigenvalue of $A$ and by $\mu_{m+1}$ its smallest positive eigenvalue. Thus there is an orthogonal $\left\{T_{t}\right\}_{t \geq 0}$-invariant splitting of $H$ using the negative eigenvalues $\left\{\mu_{1}, \mu_{2}, \cdots, \mu_{m}\right\}$ and the positive eigenvalues $\left\{\mu_{n}: n \geq m+1\right\}$ of $A$ :

$$
H=H^{+} \oplus H^{-}
$$

where $H^{+}$is a closed linear subspace of $H$ and $H^{-}$is a finite-dimensional subspace. Denote by $p^{+}: H \rightarrow H^{+}$and $p^{-}: H \rightarrow H^{-}$the corresponding projections onto $H^{+}$and $H^{-}$respectively. 
Since $H^{-}$is finite-dimensional, then $T_{t} \mid H^{-}$is invertible for each $t \geq 0$. Therefore, we can set $T_{-t}:=\left[T_{t} \mid H^{-}\right]^{-1}: H^{-} \rightarrow H^{-}$for each $t \geq 0$.

Consider the following semilinear stochastic evolution equation on $\mathrm{H}$ :

$$
\begin{aligned}
d u(t) & =[-A u(t)+F(u(t))] d t+B_{0} d W(t), \quad t \geq 0, \\
u(0) & =x \in H .
\end{aligned}
$$

In the above equation, let $F: H \rightarrow H$ be a globally Lipschitz map with Lipschitz constant $L$ :

$$
\left|F\left(v_{1}\right)-F\left(v_{2}\right)\right| \leq L\left|v_{1}-v_{2}\right|, \quad v_{1}, v_{2} \in H .
$$

Then (2.4.1) has a unique mild solution given by

$$
u(t, x)=T_{t} x+\int_{0}^{t} T_{t-s} F(u(s, x)) d s+\int_{0}^{t} T_{t-s} B_{0} d W(s), \quad t \geq 0
$$

Furthermore, if $F: H \rightarrow H$ is $C^{k, \epsilon}$, the mild solution of (2.4.2) generates a $C^{k, \epsilon}$ perfect cocycle also denoted by $u: \mathbf{R}^{+} \times H \times \Omega \rightarrow H$.

Suppose that $F: H \rightarrow H$ is globally bounded, and its Lipschitz constant $L$ satisfies

$$
L\left[\mu_{m+1}^{-1}-\mu_{m}^{-1}\right]<1
$$

Note that the above condition is automatically satisfied in the affine linear case $F \equiv 0$.

The next proposition is key to the existence and uniqueness of a stationary random point for the cocycle $(u, \theta)$ in the sense of Definition 2.1.1.

\section{Proposition 2.4.1.}

Assume the above conditions on $A, B_{0}, F$ together with (2.4.3). Then there is a unique $\mathcal{F}$-measurable map $Y: \Omega \rightarrow H$ satisfying

$$
\begin{array}{r}
Y(\omega)=\int_{-\infty}^{0} T_{-s} p^{+} F(Y(\theta(s, \omega))) d s-\int_{0}^{\infty} T_{-s} p^{-} F(Y(\theta(s, \omega))) d s \\
+(\omega) \int_{-\infty}^{0} T_{-s} p^{+} B_{0} d W(s)-(\omega) \int_{0}^{\infty} T_{-s} p^{-} B_{0} d W(s)
\end{array}
$$

for all $\omega \in \Omega$.

Proof.

We use a contraction mapping argument to show that the integral equation (2.4.4) has an $\mathcal{F}$-measurable solution $Y: \Omega \rightarrow H$.

Define the $\mathcal{F}$-measurable map $Y_{1}: \Omega \rightarrow H$ by

$$
Y_{1}(\omega):=(\omega) \int_{-\infty}^{0} T_{-s} p^{+} B_{0} d W(s)-(\omega) \int_{0}^{\infty} T_{-s} p^{-} B_{0} d W(s), \quad \omega \in \Omega .
$$


Denote by $B(\Omega, H)$ the Banach space of all (surely) bounded $\mathcal{F}$-measurable maps $Z: \Omega \rightarrow H$ given the supremum norm $\|Z\|_{\infty}:=\sup _{\omega \in \Omega}|Z(\omega)|$. Define the map $\mathcal{M}: B(\Omega, H) \rightarrow L^{0}(\Omega, H)$ by

$$
\begin{aligned}
\mathcal{M}(Z)(\omega):=\int_{-\infty}^{0} T_{-s} p^{+} & F\left(Z(\theta(s, \omega))+Y_{1}(\theta(s, \omega))\right) d s \\
& -\int_{0}^{\infty} T_{-s} p^{-} F\left(Z(\theta(s, \omega))+Y_{1}(\theta(s, \omega))\right) d s
\end{aligned}
$$

for all $Z \in B(\Omega, H)$ and all $\omega \in \Omega$.

Note first that $\mathcal{M}$ maps $B(\Omega, H)$ into itself. To see this let $Z \in B(\Omega, H)$ and $\omega \in \Omega$. Then

$$
\begin{aligned}
|\mathcal{M}(Z)(\omega)| & \leq\|F\|_{\infty}\left[\int_{-\infty}^{0}\left\|T_{-s} p^{+}\right\| d s+\int_{0}^{\infty}\left\|T_{-s} p^{-}\right\| d s\right] \\
& \leq\|F\|_{\infty}\left[\int_{-\infty}^{0} e^{s \mu_{m+1}} d s+\int_{0}^{\infty} e^{s \mu_{m}} d s\right] \\
& \leq\|F\|_{\infty}\left[\mu_{m+1}^{-1}-\mu_{m}^{-1}\right]<\infty
\end{aligned}
$$

where $\|F\|_{\infty}:=\sup _{v \in H}|F(v)|$. Hence $\mathcal{M}(Z) \in B(\Omega, H)$ for all $Z \in B(\Omega, H)$.

Secondly, $\mathcal{M}$ is a contraction. To prove this, take any $Z_{1}, Z_{2} \in B(\Omega, H)$ and $\omega \in \Omega$. Then from the definition of $\mathcal{M}$, we get

$$
\begin{aligned}
\left|\mathcal{M}\left(Z_{1}\right)(\omega)-\mathcal{M}\left(Z_{2}\right)(\omega)\right| & \leq L \int_{-\infty}^{0}\left\|T_{-s} p^{+}\right\| \cdot\left|Z_{1}(\theta(s, \omega))-Z_{2}(\theta(s, \omega))\right| d s \\
& +L \int_{0}^{\infty}\left\|T_{-s} p^{-}\right\| \cdot\left|Z_{1}(\theta(s, \omega))-Z_{2}(\theta(s, \omega))\right| d s \\
& \leq L\left\|Z_{1}-Z_{2}\right\|_{\infty}\left[\int_{-\infty}^{0}\left\|T_{-s} p^{+}\right\| d s+\int_{0}^{\infty}\left\|T_{-s} p^{-}\right\| d s\right] \\
& \leq L\left\|Z_{1}-Z_{2}\right\|_{\infty}\left[\int_{-\infty}^{0} e^{s \mu_{m+1}} d s+\int_{0}^{\infty} e^{s \mu_{m}} d s\right] \\
& =L\left[\mu_{m+1}^{-1}-\mu_{m}^{-1}\right]\left\|Z_{1}-Z_{2}\right\|_{\infty} \\
& =\mu\left\|Z_{1}-Z_{2}\right\|_{\infty}
\end{aligned}
$$

where $\mu:=L\left[\mu_{m+1}^{-1}-\mu_{m}^{-1}\right]<1$. This proves that $\mathcal{M}: B(\Omega, H) \rightarrow B(\Omega, H)$ is a contraction, and hence has a unique fixed point $Z_{0} \in B(\Omega, H)$. That is

$$
\begin{aligned}
Z_{0}(\omega):=\int_{-\infty}^{0} T_{-s} p^{+} F & \left.Z_{0}(\theta(s, \omega))+Y_{1}(\theta(s, \omega))\right) d s \\
& \quad-\int_{0}^{\infty} T_{-s} p^{-} F\left(Z_{0}(\theta(s, \omega))+Y_{1}(\theta(s, \omega))\right) d s
\end{aligned}
$$

for all $\omega \in \Omega$. Now define $Y: \Omega \rightarrow H$ by

$$
Y(\omega):=Z_{0}(\omega)+Y_{1}(\omega), \quad \omega \in \Omega .
$$

It is easy to check that $Y$ satisfies the identity (2.4.4).

Since $Z_{0}$ is uniquely determined, then so is $Y$.

The following proposition gives existence and uniqueness of a stationary point for the see $(2.4 .1)$. 


\section{Proposition 2.4.2.}

Assume all the conditions on $A, B_{0}, F$ stated in Proposition 2.4.1. Suppose that $F$ is globally bounded, globally Lipschitz and satisfies condition (2.4.3). Then the semilinear see (2.4.1) has a unique stationary point $Y: \Omega \rightarrow H$, i.e. $u(t, Y(\omega), \omega)=Y(\theta(t, \omega))$ for all $t \geq 0$ and $\omega \in \Omega$. Furthermore, $Y \in L^{p}(\Omega, H)$ for all $p \geq 1$.

Proof.

By hypotheses and Proposition 2.4.1, the integral equation (2.4.4) has a unique $\mathcal{F}$-measurable solution $Y: \Omega \rightarrow H$. Let $t \geq 0$. Using (2.4.4), it follows that

$$
\begin{aligned}
Y(\theta(t, \omega))= & \int_{-\infty}^{0} T_{-s} p^{+} F(Y(\theta(t+s, \omega))) d s-\int_{0}^{\infty} T_{-s} p^{-} F(Y(\theta(t+s, \omega))) d s \\
& +(\omega) \int_{-\infty}^{0} T_{-s} p^{+} B_{0} d W(s+t)-(\omega) \int_{0}^{\infty} T_{-s} p^{-} B_{0} d W(s+t) \\
= & \int_{-\infty}^{t} T_{t-s} p^{+} F(Y(\theta(s, \omega))) d s-\int_{t}^{\infty} T_{t-s} p^{-} F(Y(\theta(s, \omega))) d s \\
& +(\omega) \int_{-\infty}^{t} T_{t-s} p^{+} B_{0} d W(s)-(\omega) \int_{t}^{\infty} T_{t-s} p^{-} B_{0} d W(s) \\
= & T_{t}\left[\int_{-\infty}^{0} T_{-s} p^{+} F(Y(\theta(s, \omega))) d s-\int_{0}^{\infty} T_{-s} p^{-} F(Y(\theta(s, \omega))) d s\right. \\
& \left.+(\omega) \int_{-\infty}^{0} T_{-s} p^{+} B_{0} d W(s)-(\omega) \int_{0}^{\infty} T_{-s} p^{-} B_{0} d W(s)\right] \\
& +\int_{0}^{t} T_{t-s} p^{+} F\left(Y(\theta(s, \omega)) d s+\int_{0}^{t} T_{t-s} p^{-} F(Y(\theta(s, \omega))) d s\right. \\
& +(\omega) \int_{0}^{t} T_{t-s} p^{+} B_{0} d W(s)+(\omega) \int_{0}^{t} T_{t-s} p^{-} B_{0} d W(s) \\
= & T_{t} Y(\omega)+\int_{0}^{t} T_{t-s} F(Y(\theta(s, \omega))) d s+(\omega) \int_{0}^{t} T_{t-s} B_{0} d W(s) .
\end{aligned}
$$

This gives

$$
Y(\theta(t, \omega))=T_{t} Y(\omega)+\int_{0}^{t} T_{t-s} F(Y(\theta(s, \omega))) d s+(\omega) \int_{0}^{t} T_{t-s} B_{0} d W(s)
$$

for all $t \geq 0$. Therefore, $Y(\theta(t, \omega)), t \geq 0, \omega \in \Omega$, is a stationary solution of (2.4.2) (with $x=Y(\omega)$ ). Since $u(t, Y(\omega), \omega), t \geq 0, \omega \in \Omega$, is also a solution of (2.4.2), then by uniqueness of the solution to (2.4.2), we must have

$$
u(t, Y(\omega), \omega)=Y(\theta(t, \omega))
$$

for all $t \geq 0$ and all $\omega \in \Omega$. Hence $Y$ is a stationary point for the see (2.4.1).

The stationary point for (2.4.1) is unique (within the class of $\mathcal{F}$-measurable maps $\Omega \rightarrow H$ ). To see this, it is sufficient to observe that the above computation shows that every stationary point 
of (2.4.1) is a solution of the integral equation (2.4.4). Uniqueness of the stationary solution then follows from Proposition 2.4.1.

In view of the proof of Proposition 2.4.1, the last assertion of Proposition 2.4.2 follows from the fact that $Y_{1} \in L^{p}(\Omega, H)$ for all $p \geq 1$ and $Z_{0} \in L^{\infty}(\Omega, H)$.

The existence of local stable and unstable manifolds near a stationary point of the affine stochastic evolution equation (2.4.1) follows from a straightforward modification of the proof of Theorem 2.4.1 in the next section.

(b) Semilinear stochastic evolution equations: Linear noise

Here we recall the setting and hypotheses leading to Theorem 1.2.6.

We will prove the existence of local stable and unstable manifolds for semiflows generated by mild solutions of semilinear stochastic evolution equations of the form:

$$
\left.\begin{array}{rl}
d u(t) & =-A u(t) d t+F(u(t)) d t+B u(t) d W(t), \quad t>0, \\
u(0) & =x \in H .
\end{array}\right\}
$$

In the above equation $A: D(A) \subset H \rightarrow H$ is a closed linear operator on a separable real Hilbert space $H$. Assume that $A$ has a complete orthonormal system of eigenvectors $\left\{e_{n}: n \geq\right.$ $1\}$ with corresponding positive eigenvalues $\left\{\mu_{n}, n \geq 1\right\}$; i.e., $A e_{n}=\mu_{n} e_{n}, n \geq 1$. Suppose $-A$ generates a strongly continuous semigroup of bounded linear operators $T_{t}: H \rightarrow H, t \geq 0$. Let $E$ be a separable real Hilbert space. Suppose $W(t), t \geq 0$, is $E$-valued cylindrical Brownian motion defined on the canonical filtered Wiener space $\left(\Omega, \mathcal{F},\left(\mathcal{F}_{t}\right)_{t \geq 0}, P\right)$ and with a separable covariance Hilbert space $K$, where $K \subset E$ is a Hilbert-Schmidt embedding. That is, $\Omega$ is the space of all continuous paths $\omega: \mathbf{R} \rightarrow E$ such that $\omega(0)=0$ with the compact open topology, $\mathcal{F}$ is its Borel $\sigma$-field, $\mathcal{F}_{t}$ is the sub- $\sigma$-field generated by all evaluations $\Omega \ni \omega \mapsto \omega(u) \in E, u \leq t$, and $P$ is Wiener measure on $\Omega$. The Brownian motion is given by

$$
W(t, \omega):=\omega(t), \quad \omega \in \Omega, t \in \mathbf{R},
$$

and may be represented by

$$
W(t)=\sum_{k=1}^{\infty} W^{k}(t) f_{k}, \quad t \in \mathbf{R},
$$

where $\left\{f_{k}: k \geq 1\right\}$ is a complete orthonormal basis of $K$, and the $W^{k}, k \geq 1$, are standard independent one-dimensional Wiener processes ([D-Z.1], Chapter 4).

Suppose $B: H \rightarrow L_{2}(K, H)$ is a bounded linear operator. The stochastic integral in (2.4.5) is defined in the sense of ([D-Z.1], Chapter 4).

Assume the hypotheses of Theorem 1.2.4.

We will denote by $\theta: \mathbf{R} \times \Omega \rightarrow \Omega$ the standard $P$-preserving ergodic Wiener shift on $\Omega$ :

$$
\theta(t, \omega)(s):=\omega(t+s)-\omega(t), \quad t, s \in \mathbf{R} .
$$


Let $L(H)$ be the Banach space of all bounded linear operators $H \rightarrow H$ given the uniform operator norm $\|\cdot\|$. Denote by $L_{2}(H) \subset L(H)$ the Hilbert space of all Hilbert-Schmidt operators $S: H \rightarrow H$.

Suppose $F: H \rightarrow H$ is a (Fréchet) $C^{k, \epsilon}(k \geq 1, \epsilon \in(0,1])$ non-linear map satisfying the following Lipschitz and linear growth hypotheses:

$$
\left.\begin{array}{rl}
|F(v)| & \leq C(1+|v|), \quad v \in H \\
\left|F\left(v_{1}\right)-F\left(v_{2}\right)\right| & \leq L_{n}\left|v_{1}-v_{2}\right|, \quad v_{i} \in H,\left|v_{i}\right| \leq n, i=1,2,
\end{array}\right\}
$$

for some positive constants $C, L_{n}, n \geq 1$.

The mild solutions of the see (2.4.5) generate a $C^{k, \epsilon}(k \geq 1, \epsilon \in(0,1])$ perfect cocycle $(U, \theta)$ on $H$, satisfying all the assertions of Theorem 1.2.6.

Under the above conditions, one gets the following stable manifold theorem for hyperbolic stationary trajectories of the see (2.4.5).

\section{Theorem 2.4.1.}

Assume the above hypotheses on the coefficients of the see (2.4.5). Assume that the stochastic semiflow $U: \mathbf{R}^{+} \times H \times \Omega \rightarrow H$ generated by mild solutions of (2.4.5) has a hyperbolic stationary point $Y: \Omega \rightarrow H$ such that $E \log ^{+}|Y|<\infty$. Then $(U, \theta)$ has a perfect family of $C^{k, \epsilon}$ local stable and unstable manifolds satisfying all the assertions of Theorem 2.2.1.

Proof.

One first checks the estimate

$$
\int_{\Omega} \log ^{+} \sup _{0 \leq t_{1}, t_{2} \leq a}\left\|U\left(t_{2}, Y\left(\theta\left(t_{1}, \omega\right)\right)+(\cdot), \theta\left(t_{1}, \omega\right)\right)\right\|_{k, \epsilon} d P(\omega)<\infty
$$

for any fixed $0<\rho, a<\infty, k \geq 1$ and $\epsilon \in(0,1]$. This estimate follows from the integrability condition on $Y$ and assertion (vi) of Theorem 1.2.6. The conclusion of Theorem 2.4.1 now follows immediately from Theorem 2.2.1.

(c) Semilinear parabolic spde's: Lipschitz nonlinearity

Consider the Laplacian

$$
\Delta:=\frac{1}{2} \sum_{i, j=1}^{d} \frac{\partial^{2}}{\partial \xi_{i}^{2}}
$$

defined on a smooth bounded domain $\mathcal{D}$ in $\mathbf{R}^{d}$, with a smooth boundary $\partial \mathcal{D}$ with zero Dirichlet boundary conditions. Assume that $f: \mathbf{R} \rightarrow \mathbf{R}$ is a $C_{b}^{\infty}$ function and let $d \xi$ be Lebesgue measure on $\mathbf{R}^{d}$. Let $W_{i}, i \geq 1$, be independent one-dimensional standard Brownian motions with $W_{i}(0)=0$ defined on the canonical filtered Wiener space $\left(\Omega, \mathcal{F}, P,\left(\mathcal{F}_{t}\right)_{t \in \mathbf{R}}\right)$. Let $\theta$ denote the Brownian shift on $\Omega:=C\left(\mathbf{R}, \mathbf{R}^{\infty}\right)$. Recall that the Sobolev space $H_{0}^{k}(\mathcal{D})$ is the completion of $C_{0}^{\infty}(\mathcal{D}, \mathbf{R})$ under the Sobolev norm

$$
\|u\|_{H_{0}^{k}(\mathcal{D})}^{2}:=\sum_{|\alpha| \leq k} \int_{\mathcal{D}}\left|D^{\alpha} u(\xi)\right|^{2} d \xi
$$


Suppose further that $\sigma_{i} \in H_{0}^{s}(\mathcal{D}), i \geq 1$, and the series $\sum_{i=1}^{\infty}\left\|\sigma_{i}\right\|_{H_{0}^{s}}^{2}$ converges, where $s>k+\frac{d}{2}>d$. By Theorem 1.3.5, weak solutions of the initial-value problem:

$$
\left.\begin{array}{rl}
d u(t) & =\frac{1}{2} \Delta u(t) d t+f(u(t)) d t+\sum_{i=1}^{\infty} \sigma_{i} u(t) d W_{i}(t), \quad t>0 \\
u(0) & =\psi \in H_{0}^{k}(\mathcal{D})
\end{array}\right\}
$$

give a perfect smooth cocycle $(U, \theta)$ on the Sobolev space $H_{0}^{k}(\mathcal{D})$ which satisfies all the assertions of Theorem 1.3.5. Applying Theorem 2.2.1, we get the following stable manifold theorem for the spde (2.4.9):

\section{Theorem 2.4.2.}

Assume the above hypotheses on the coefficients of the spde (2.4.9). Assume that the stochastic semiflow $U: \mathbf{R}^{+} \times H_{0}^{k}(\mathcal{D}) \times \Omega \rightarrow H_{0}^{k}(\mathcal{D})$ generated by weak solutions of (2.4.9) has a hyperbolic stationary point $Y: \Omega \rightarrow H_{0}^{k}(\mathcal{D})$ such that $E \log ^{+}\|Y\|_{H_{0}^{k}}<\infty$. Then $(U, \theta)$ has a perfect family of $C^{\infty}$ local stable and unstable manifolds in $H_{0}^{k}(\mathcal{D})$ satisfying all the assertions of Theorem 2.2.1.

(d) Stochastic reaction diffusion equations: dissipative nonlinearity

In section 1.4 (a), we constructed a $C^{1}$ stochastic semiflow on the Hilbert space $H:=L^{2}(\mathcal{D})$ for a stochastic reaction-diffusion equation

$$
d u=\nu \Delta u d t+u\left(1-|u|^{\alpha}\right) d t+\sum_{i=1}^{\infty} \sigma_{i} u(t) d W_{i}(t)
$$

defined on a bounded domain $\mathcal{D} \subset \mathbf{R}^{d}$ with a smooth boundary $\partial \mathcal{D}$. In (2.4.10), the Laplacian on $\mathcal{D}$ is denoted by $\Delta$, and we impose Dirichlet boundary conditions on $\partial \mathcal{D}$. The $W_{i}, i \geq 1$, are independent one-dimensional standard Brownian motions and the series $\sum_{i=1}^{\infty}\left\|\sigma_{i}\right\|_{H_{0}^{s}}^{2}$ converges for $s>2+\frac{d}{2}$. The dissipative term yields the existence of a unique stationary solution of (2.4.10) under a suitable choice of the parameter $\nu$ ([D-Z.2]).

In view of the estimates in Theorem 1.4.1 and Theorem 2.2.1, one gets the following:

\section{Theorem 2.4.3.}

Assume the above hypotheses on the coefficients of the spde (2.4.10). Let $\alpha<\frac{4}{d}$. Assume that the stochastic semiflow $U: \mathbf{R}^{+} \times L^{2}(\mathcal{D}) \times \Omega \rightarrow L^{2}(\mathcal{D})$ generated by mild solutions of $(2.4 .10)$ has a hyperbolic stationary point $Y: \Omega \rightarrow L^{2}(\mathcal{D})$ such that $E \log ^{+}\|Y\|_{L^{2}}<\infty$. Then $(U, \theta)$ has a perfect family of $C^{1}$ local stable and unstable manifolds in $L^{2}(\mathcal{D})$ satisfying all the assertions of Theorem 2.2.1. 
Remarks.

(i) The results in Sections (c) and (d) hold if the Euclidean domain $\mathcal{D}$ is replaced by a compact smooth $d$-dimensional Riemannian manifold $M$ (possibly with a smooth boundary $\partial M$ ).

(ii) We conjecture that Theorem 2.4.3 still holds (but with Lipschitz stable/unstable manifolds) if the dissipative term $u\left(1-|u|^{\alpha}\right)$ is replaced by a more general one of the form $F(u):=f \circ u$, where $f: \mathbf{R} \rightarrow \mathbf{R}$ is a $C^{1}$ function satisfying the following classical estimates:

$$
-c_{1}-\alpha_{1}|x|^{p} \leq f(x) x \leq c_{1}-\alpha_{2}|x|^{p}, \quad f^{\prime}(x) \leq c_{2},
$$

for all $x \in \mathbf{R}$, with $c_{1}, c_{2}, \alpha_{1}, \alpha_{2}$ positive constants, and $p$ any integer greater than 2 .

(iii) Is it true that the stochastic flow and the local stable/unstable manifolds in Theorem 2.4.3 are of class $C^{2}$ ?

(e) Stochastic Burgers equation: additive noise

The existence of a $C^{1}$ stochastic semiflow on $L^{2}([0,1])$ for Burgers equation

$$
d u+u \frac{\partial u}{\partial \xi} d t=\nu \Delta u d t+d W(t), \quad t>0, \nu>0
$$

was established in Part 1 of this paper, where $W(t), t>0$, is an infinite dimensional Brownian motion on $L^{2}[0,1]$. See Theorem 1.4.3.

Under extra spatial smoothness hypotheses on the noise, viz. $W(t, \cdot) \in C^{3}([0,1])$, Burgers equation (2.4.11) admits a unique stationary point ([Si]). More generally, with our weaker condition on the noise $W$ (Section 1.4 (b)), we stipulate that equation (2.4.11) has a hyperbolic stationary point. In this case, we get the following result:

\section{Theorem 2.4.4.}

Assume the hypotheses of Theorem 1.4.3 on the coefficients of Burgers spde (2.4.11). Assume that the stochastic semiflow $U: \mathbf{R}^{+} \times L^{2}([0,1]) \times \Omega \rightarrow L^{2}([0,1])$ generated by mild solutions of (2.4.11) has a hyperbolic stationary point $Y: \Omega \rightarrow L^{2}([0,1])$ such that $E \log ^{+}\|Y\|_{L^{2}}<\infty$. Then $(U, \theta)$ has a perfect family of $C^{1}$ local stable and unstable manifolds in $L^{2}([0,1])$ satisfying all the assertions of Theorem 2.2.1.

Note that hyperbolicity of the stationary point in Theorem 2.4.4 is in the sense of Definition (2.1.3). Theorems 2.1.1 and 1.4.3 imply that the Lyapunov spectrum for the linearization of (2.4.11) exists and is discrete for any viscosity $\nu>0$. When $W$ is $C^{3}$ in the space variable, it known that for any $C^{2}$ initial condition, the solution $u(t)$ of (2.4.11) converges to the stationary solution for any positive viscosity $\nu>0([\mathrm{Si}])$. It is therefore easy to see that the stable manifold is the whole of $L^{2}[0,1]$.

The case of sufficiently large viscosity and rough noise $W(t) \in L^{2}([0,1])$ is currently being studied ([L-Z]). This work shows that (2.4.11) admits a unique globally exponentially stable stationary point in this case. So in this (somewhat non-generic) case, the unstable manifold consists 
of the single random point $Y(\omega) \in L^{2}([0,1])$, and the non-linear cocycle $U$ will approach $Y(\omega)$ with exponential speed less than or equal to the top Lyapunov exponent $\lambda_{1}$ of the linearized Burgers equation.

We conjecture that the assertions in the above paragraph still hold for any viscosity $\nu>0$ (cf. [D-Z.2], Theorem 14.4.4). Further analysis of the Lyapunov spectrum for (2.4.11) (in the cases of small and zero viscosity $\nu$ ) is postponed to a future project.

\section{Acknowledgments}

The authors would like to thank Prof. B. Øksendal for inviting them to Oslo in the summer of 2000 where the project was started. They are also grateful to Prof. K.D. Elworthy for inviting them to Warwick on numerous occasions especially during the Warwick SPDE's Symposium 2000/2001 so that they may have opportunities to meet; to Prof. A. Truman for inviting them to the International Workshop of Probabilistic Methods in Fluids at Swansea in April 2002 where preliminary versions of the results were announced; and to the organizers of ICM 2002 and First Sino-German Stochastic Analysis Conference in Beijing in August 2002 where the results were presented. The authors would also like to thank the referees for useful comments. 


\section{REFERENCES}

[A] Arnold, L., Random Dynamical Systems, Springer-Verlag (1998).

[A-S] Arnold, L., and Scheutzow, M. K. R., Perfect cocycles through stochastic differential equations, Probab. Th. Rel. Fields, 101, (1995), 65-88.

[B-C-J] Bertini, L., Cancrin, N. and Jona-Lasinio, G.: The stochastic Burgers equation. Commum. Math. Phys., 165 (1994), 211-232.

[B-C-F] Brzezniak, Z., Capinski, M, and Flandoli, F., Stochastic Navier-Stokes equations with multiplicative noise, Stochastic Analysis and Applications, 10 (1992), 523-532.

[B-F] Brzezniak, Z., and Flandoli, F., Regularity of solutions and random evolution operator for stochastic parabolic equations, In: Stochastic partial differential equations and applications (Trento, 1990), pp54-71, Pitman Res. Notes Math. Ser., 268, Longman Sci. Tech., Harlow, 1992.

[B-F.1] Bensoussan, A. and Flandoli, F., Stochastic inertial manifold. Stochastics Stochastics Rep. 53 (1995), no. 1-2, 13-39.

[C-K-S] Carballo, T., Kloeden, P.E., Schmalfuss, B., Exponentially stable stationary solution for stochastic evolution equations and their perturbation, Appl. Math. Optim., 50(2004), 183207.

[C] Carverhill, A., Flows of stochastic dynamical systems: ergodic theory, Stochastics, 14(1985), 273-317.

[C-V] Castaing, C., and Valadier, M., Convex Analysis and Measurable Multifunctions, Lecture Notes in Mathematics, vol. 580, Springer-Verlag, Berlin-Heidelberg-New York (1977).

[Co] Cohn, D. L., Measure Theory, Birkhäuser (1980).

[D-D-T] Da Prato, G., Debusche, A. and Temam, R.: Stochastic Burgers equation. Nonlinear Differential Eq. Appl, 1 (1994), 389.

[D-Z.1] Da Prato, G., and Zabczyk, J., Stochastic Equations in Infinite Dimensions, Cambridge University Press (1992).

[D-Z.2] Da Prato, G., and Zabczyk, J., Ergodicity for Infinite Dimensional Systems, Cambridge University Press (1996).

[D-T-Z] Davies, I. M., Truman, A., Zhao, H.Z., Stochastic generalized KPP equations, Proc. $R$. Soc. Edinb., Vol. 126A (1996), No. 5, 957-84.

[D-L-S.1] Duan, J., Lu, K. and Schmalfuss, B., Invariant manifolds for stochastic partial differential equations, Annals of Probability, 31(2003), 2109-2135.

[D-L-S.1] Duan, J., Lu, K. and Schmalfuss, B., Stable and unstable manifolds for stochastic partial differential equations, J. Dynamics and Diff. Eqns, 2004, Vol. 16, No. 4, 949-972. 
[E-V] E, W., Vanden Eijnden, E., Statistical theory for the stochastic Burgers equation in the inviscid limit, Commun. Pure Appl. Math. 53(2000), 852-901.

[E-H] Eckmann, J.-P., Hairer, M., Invariant measures for stochastic partial differential equations in unbounded domains, Nonlinearity, Vol. 14 (2001), 133-151.

[E-Z] Elworthy, K. D. and Zhao, H. Z., The propagation of travelling waves for stochastic generalized KPP equations, Mathl. and Comput. Modelling, Vol. 20 (1994), No.4/5, 131-66.

[F.1] Flandoli, F., Regularity theory and stochastic flows for parabolic SPDE's, Stochastics Monographs, 9, Gordon and Breach Science Publishers, Yverdon (1995).

[F.2] Flandoli, F., Stochastic flows for nonlinear second-order parabolic SPDE, Ann. Probab. 24 (1996), no. 2, 547-558.

[F-S] Flandoli, F., and Schaumlöffel, K.-U., Stochastic parabolic equations in bounded domains: random evolution operator and Lyapunov exponents, Stochastics and Stochastics Reports 29 (1990), no. 4, 461-485.

[F-S] Flandoli, F., and Schaumlöffel, K.-U., A multiplicative ergodic theorem with applications to a first order stochastic hyperbolic equation in a bounded domain, Stochastics and Stochastics Reports, 34 (1991), no. 3-4, 241-255.

[Fr] Freidlin, M., Functional integration and partial differential equations, Princeton University Press, Princeton, New Jersey (1985).

[Fri] Friedman, A., Partial differential equations of parabolic type, Prentice-Hall, Inc., Englewood Cliffs, N. J. (1964).

[H-L-O-U-Z] Holden, H., Lindstrom, T., Oksendal, B., Uboe, J. and Zhang, T. S.: The Burgers' equation with a noise force and the stochastic heat equations. Comm. PDE., 19 (1994), 119-141.

[I-W] Ikeda, N., and Watanabe, S., Stochastic Differential Equations and Diffusion Processes, Second Edition, North-Holland-Kodansha (1989).

[Ku] Kunita, H., Stochastic Flows and Stochastic Differential Equations, Cambridge University Press, Cambridge, New York, Melbourne, Sydney (1990).

[L-S-U] Ladyzenskaja, O. A., Solonnikov, V. A. and Uralceva, N. N., Linear and quasi-linear equations of parabolic type, Translations of Mathematical Monographs, Vol. 23, American Mathematical Society (1968).

[L-Z] Liu, Y. and Zhao, H.Z., The stochastic Burgers integral equations and the stationary solutions of stochastic Burgers equations, in preparation.

[Ly] Lyapunov, M. A., Problèm général de la stablité du mouvement, Annales Dac. Sciences Toulouse, 9(1907) (Translation of the Russian edition, Kharkov 1892). Reprinted by Princeton University Press, Princeton, N.J. 1949 and 1952.

[Mo.1] Mohammed, S.-E.A., Stochastic Functional Differential Equations, Research Notes in Mathematics, no. 99, Pitman Advanced Publishing Program, Boston-London-Melbourne (1984). 
[Mo.2] Mohammed, S.-E. A., Non-Linear Flows for Linear Stochastic Delay Equations, Stochastics, Vol. 17 \#3, (1987), 207-212.

[M-S.1] Mohammed, S.-E. A., and Scheutzow, M. K. R., The stable manifold theorem for non-linear stochastic systems with memory. Part I: Existence of the semiflow. Part II: The local stable manifold theorem" (preprints) (2001).

[M-S.2] Mohammed, S.-E. A., and Scheutzow, M. K. R., The stable manifold theorem for stochastic differential equations, The Annals of Probability, Vol. 27, No. 2, (1999), 615-652.

[M-S.3] Mohammed, S.-E. A., and Scheutzow, M. K. R., Spatial estimates for stochastic flows in Euclidean space, The Annals of Probability, Vol. 26, No. 1, (1998), 56-77.

[M-S.4] Mohammed, S.-E. A., and Scheutzow, M. K. R.,Lyapunov exponents of linear and stochastic functional differential equations driven by semimartingales, Part I: The multiplicative ergodic theory, Annals of Institute of Henri Poincare, Vol. 32, 1, (1996), 69-105.

[O-V-Z] Oksendal, B., Väge, G. and Zhao, H. Z.,Two properties of stochastic KPP equations: ergodicity and pathwise property, Nonlinearity, Vol. 14 (2001), 639-662.

[O] Oseledec, V. I., A multiplicative ergodic theorem. Lyapunov characteristic numbers for dynamical systems, Trudy Moskov. Mat. Obšč. 19 (1968), 179-210. English transl. Trans. Moscow Math. Soc. 19 (1968), 197-221.

[Pr] Protter, Ph. E., Stochastic Integration and Stochastic Differential Equations: A New Approach, Springer (1990).

[Ro] Robinson, J. C., Infinite-Dimensional Dynamical Systems. An Introduction to Dissipative Parabolic PDE's and the Theory of Global Attractors, Cambridge Texts in Applied Mathematics, Cambridge University Press (2001).

[Ru.1] Ruelle, D., Ergodic theory of differentiable dynamical systems, Publ. Math. Inst. Hautes Etud. Sci. (1979), 275-306.

[Ru.2] Ruelle, D., Characteristic exponents and invariant manifolds in Hilbert space, Annals of Math. 115 (1982), 243-290.

[Sc] Schmalfuss, B., A random fixed point theorem and the random graph transformation, $J$. Math. Anal. Appli., 225(1998), 91-113.

[Si] Sinai, Ya. G., Burgers system driven by a periodic stochastic flow, In: Itô's stochastic calculus and probability theory, Springer, Tokyo (1996), 347-353.

[Sk] Skorohod, A. V., Random Linear Operators, Riedel (1984).

[Ta] Taylor, M. E., Partial Differential Equations III Nonlinear Equations, Springer (1996).

[Te] Temam, R., Infinite-Dimensional Dynamical Systems in Mechanics and Physics, SpringerVerlag (1988).

[Tw] Twardowska, K., An extension of the Wong-Zakai theorem for stochastic equations in Hilbert spaces, Stochastic Anal. Appl. 10 (1992), no. 4, 471-500. 
[T-Za] Tribe, R., Zaboronski, O., On the large time asymptotics of decaying Burgers turbulence., Comm. Math. Phys., 212 (2000), 415-436.

[T-Z] Truman, A., Zhao, H. Z., Stochastic Burgers' equations and their semi-classical expansions, Comm. Math. Phys., 194 (1998), 231-248.

[Wa] Walsh, J. B., An Introduction to stochastic partial differential equations, in: École d'eté de Probabilité de Saint Flour, XIV, ed. P.L. Hennequim, Lecture Notes in Mathematics No. 1180, pp.265-439, Springer-Verlag, (1984).

[Wan] Wanner, T., Linearization of random dynamical systems, in: Dynamics Reported, Vol. 4, pp. 203-269, Springer-Verlag, Berlin-Heidelberg-New York, (1995).

[Z-Z] Zhang, Q., Zhao, H. Z., Pathwise stationary solutions of stochastic partial differential equations and backward doubly stochastic differential equations on infinite horizon, Preprint.

Salah-Eldin A. Mohammed

Department of Mathematics,

Southern Illinois University at Carbondale,

Carbondale, Illinois 62901.

Email: salah@sfde.math.siu.edu

Web page: http://sfde.math.siu.edu

Tusheng Zhang

Department of Mathematics

University of Manchester,

Oxford Road, Manchester M13 9PL,

UK.

Email: tzhang@math.man.ac.uk

Huaizhong Zhao

Department of Mathematical Sciences

Loughborough University,

LE11 3TU,

UK.

Email: H.Zhao@lboro.ac.uk 